\title{
Salt tolerance strategies of the ancient Andean crop quinoa
}

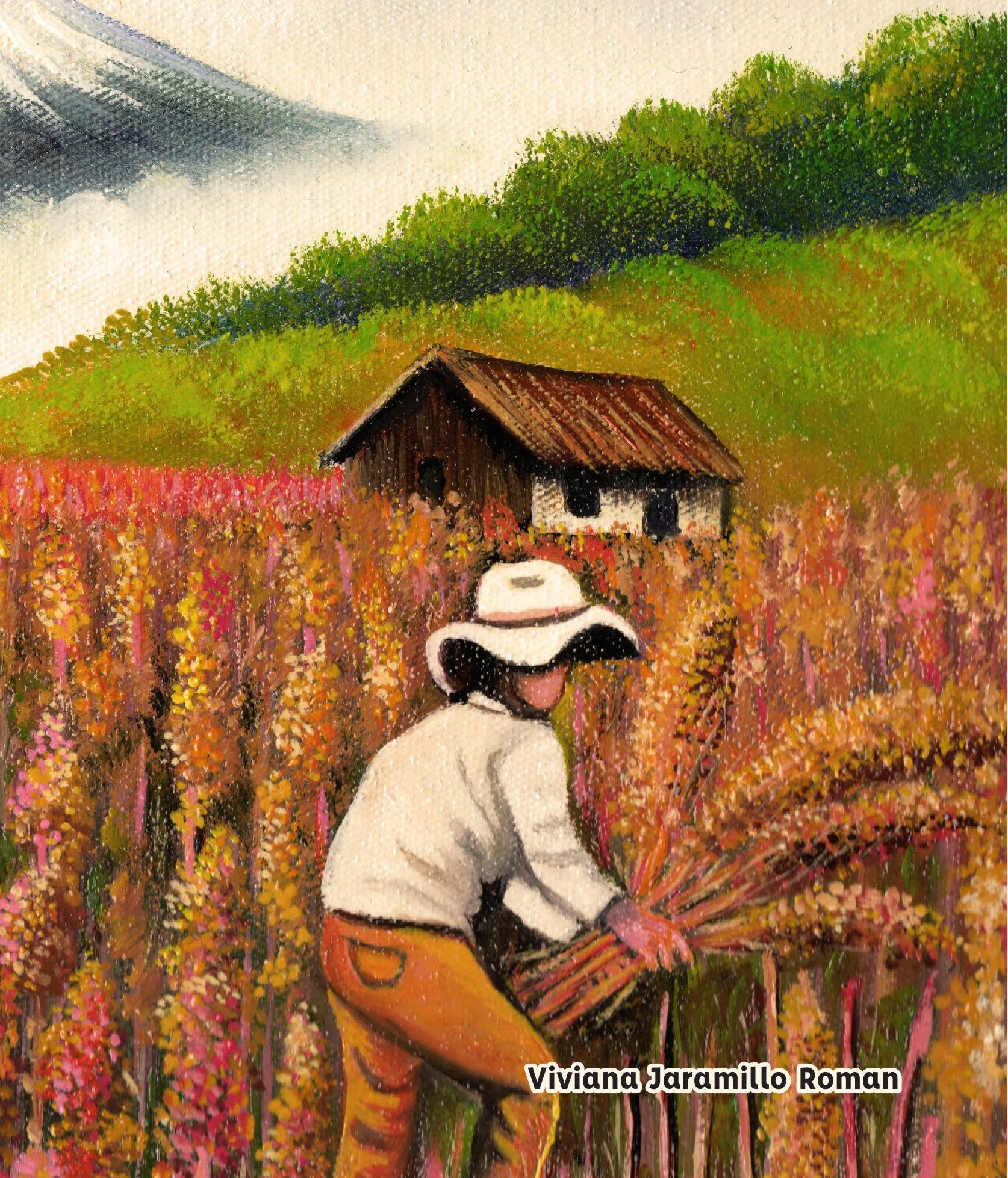




\section{Propositions}

1. $\mathrm{K}^{+}$accumulation is more a survival than a salt tolerance mechanism in quinoa.

(this thesis)

2. Epidermal bladder cells are not, by definition, salt bladders. (this thesis)

3. Bureaucracy around the Nagoya Protocol hampers scientific development, protection and fair sharing of genetic resources.

4. The development of dietary supplements should only be research-driven.

5. A new Green Revolution focused on novel, resilient and resourceuse-efficient crops is needed.

6. Physical distancing because of COVID-19 has increased the social divide in many countries.

7. The \#MeToo movement should lead to sexual violence policy reforms worldwide.

Propositions belonging to the thesis, entitled

Salt tolerance strategies of the ancient Andean crop quinoa

Viviana Jaramillo Roman

Wageningen, 19 January 2021 



\section{Salt tolerance strategies of the ancient Andean crop quinoa}

Viviana Jaramillo Roman 


\section{Thesis committee}

\section{Promotor}

Prof. Dr R.G.F. Visser

Professor of Plant Breeding

Wageningen University \& Research

\section{Co-promotors}

Dr C.G. van der Linden

Research Group Leader, Plant Breeding

Wageningen University \& Research

Dr E.N. van Loo

Senior Scientist, Plant Breeding

Wageningen University \& Research

\section{Other members}

Dr S. Schmöckel, University of Hohenheim, Germany

Prof. Dr N.P.R. Anten, Wageningen University \& Research

Dr R.B. Karlova, Wageningen University \& Research

Prof. Dr L.A.C.J. Voesenek, Utrecht University

This research was conducted under the auspices of the Graduate School of Experimental Plant Sciences (EPS) 


\section{Salt tolerance strategies of the ancient Andean crop quinoa}

\section{Viviana Jaramillo Roman}

\section{Thesis}

submitted in fulfilment of the requirements for the degree of doctor at Wageningen University

by the authority of the Rector Magnificus,

Prof. Dr A.P.J. Mol, in the presence of the

Thesis Committee appointed by the Academic Board to be defended in public

on Tuesday 19 January 2021 at 4 p.m. in the Aula. 
Viviana Jaramillo Roman

Salt tolerance strategies of the ancient Andean crop quinoa 262 pages.

$\mathrm{PhD}$ thesis, Wageningen University, Wageningen, the Netherlands (2021) With references, with summary in English

ISBN 978-94-6395-624-6

DOI $10.18174 / 535249$ 
To my grandima,

for the best quinoa soap and for being a woman ahead of her time.

$\mathcal{A}$ mi abuelita, por la mejor sopa de quínua y por ser una mujer adelantada a su época. 



\section{Table of Contents}

Chapter 1

General introduction

Chapter 2

Differential responses to salt stress in ion dynamics, growth and seed yield of European quinoa varieties

\section{Chapter 3}

Cell wall modifications of Chenopodium quinoa in response to severe salt stress

\section{Chapter 4}

High-resolution analysis of growth and transpiration of quinoa under saline conditions

Chapter 5

Genetic dissection of salt tolerance traits in Chenopodium quinoa

Chapter 6

Genetic diversity of Inter-Andean Valley Ecuadorian quinoa for salt tolerance

Chapter 7

General discussion

References

Summary

Resumen

246

Acknowledgements 250

About the author 256 




\section{The challenge of soil salinization}

The world's surface land occupies around 13.2 billion ha of which 1.5 billion ha are cultivated. Of the total cultivated land, $227 \mathrm{Mha}$ are irrigated and from those, $20 \%$ are salt-affected. Of the non-irrigated cultivated land, an additional $2.5 \%$ also suffers from salinity, resulting in more than a billion-ha in total (Ivushkin et al. 2019b; Srivastava et al. 2019; Tanji 2002). Soil salinization is the condition when soluble salts accumulate in the soil to a level that has a negative impact on the growth and development of plants, environmental health, and/or economic welfare (Rengasamy 2006; Srivastava et al. 2019). More than 100 countries struggle with soil salinization that limits crop productivity, causes substantial economic losses and threatens the current and future global food security (Tanji 2002). The distribution of salt-affected land in the world is depicted in Figure 1.

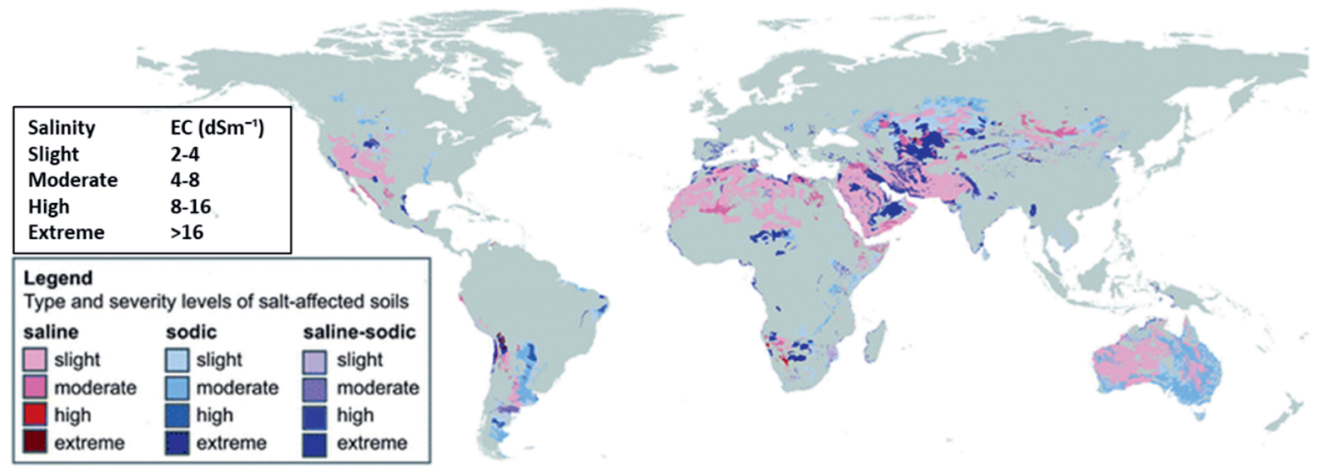

Figure 1. Global salt-affected soils by salt and severity. Adapted from (Wicke et al. 2011).

Salinization can be primary or secondary. Primary salinization occurs when salinity develops due to natural processes, mainly by the intrusion of ocean water into coastal areas and rivers followed by evapotranspiration, or by the rise of groundwater (Daliakopoulos et al. 2016). Secondary salinization has an anthropological origin caused by diverse factors such as the application of chemical fertilizers, deforestation, and salt-rich water irrigation (Srivastava et al. 2019).

Unfortunately, soil salinization is an expanding challenge strongly associated with climate change. Sea level rise in combination with drought and groundwater exploitation is expected to increase the seawater intrusion into coastal areas; temperature rise with a consequent increase in evapotranspiration is likely to increase salinization especially in arid and semi-arid areas; abandonment of saltaffected agricultural land will likely worsen the condition of those areas; and finally, 
regular drought periods will increase the need of irrigated agriculture that mostly relies on poor-quality water sources (Daliakopoulos et al. 2016).

\section{Consequences of salinity for the soil, environment, agriculture and economic welfare}

Salinity directly affects physical properties of the soil. It promotes soil dispersion and causes the conversion of soil into a cement-like structure with reduced aeration and low hydraulic capacity. In addition, saline soils are often poor in organic matter and have an imbalance in mineral content such as low magnesium and calcium (Srivastava et al. 2019). It is often associated with a decline in biodiversity through habitat fragmentation, loss of non-tolerant species and changes in the ecosystem equilibrium. It affects the life of rural communities around the globe (Srivastava et al. 2019). It has been identified as one of the most serious environmental factors limiting the productivity of crops. The economic costs of soil salinization are difficult to estimate and include complex socioeconomic interactions. Direct costs are associated with losses in crop yields and expensive remediation investments for the recovery of affected land, while indirect costs include the abandonment of agricultural areas causing unemployment plus the loss of biodiversity and environmental services from degraded lands (Qadir et al. 2014).

The rapid expansion of soil salinization and its serious consequences to economy and food security are a strong motivation to understand how salinity affects plant growth and why salt stress tolerance is imperative for the development of sustainable agriculture.

\section{A brief history of salinity and plants}

Earth is a salty planet. Life presumably arose in "primitive oceans" (with similar or even higher salinity than the current oceans), so the cells of early life forms on our planet were adapted to salinity. Plant terrestrialization is thought to have occurred several times, and phylogenetic evidence suggests that the ancestors of land plants belonged to charophyte algae, and were most probably fresh/brackish water-adapted organisms (Flowers et al. 2010). This origin might explain why many land plants can withstand slight/moderate salinity, but only 1-2 \% of all the higher plant species survive salinities close to seawater (Volkov and Beilby 2017).

Since the appearance of primitive plants, climate and geography have changed immensely, creating all kinds of new habitats that plants were able to colonize. The environmental pressure from those niches characterized by high salinity, such as coastal environments, salt marshes, salt lakes and arid areas, must 
have been the evolutive driver for the appearance and successfulness of halophytes (Bromham 2014).

Halophytes are plant species that can complete their life cycle under high salt concentrations $(\geq 200 \mathrm{mM} \mathrm{NaCl}$, or similar conditions in their natural environments) (Flowers and Colmer 2008). Halophytes are taxonomically widespread across all the families of flowering plants and have evolved independently in many lineages; it is presumed that halophytic plants evolved no less than 59 independent times (Cheeseman 2015).

Salt stress affects the performance of plants in several ways. To start, the presence of ions in the growth medium reduces its water potential, which affects water uptake. As a consequence, plant turgor is reduced as well as leaf expansion and growth, stomatal closure is promoted and with that the photosynthesis rate decreases (Shabala and Munns 2017). Until a certain threshold, plants are able to adapt to a lowered water potential (osmotic adjustment) mostly by the synthesis of organic osmolytes, or (especially in halophytes) by the accumulation of inorganic ions, mainly $\mathrm{Na}^{+}$and $\mathrm{Cl}^{-}$. Plants use two strategies to deal with high ion concentrations in the soil at varying degrees: avoiding ion uptake, excluding $\mathrm{Na}^{+}$and $\mathrm{Cl}^{-}$from leaves and relying on organic solutes for osmotic adjustment (ion exclusion); or taking up ions and sequestering them into the vacuole, to be used as osmotica (tissue tolerance) (Munns and Gilliham 2015). When excessive amounts of ions enter the transpiration stream and reach (transpiring) leaves, they may cause cytotoxic damage and osmotic imbalances within tissues (Morton et al. 2019). Additionally, salt stress usually induces oxidative stress due to the generation of reactive oxygen species (ROS) (Isayenkov and Maathuis 2019).

Mechanisms have evolved in all plants to be able to withstand the presence of salts in the soil, but the salinity level that a plant species is able to tolerate varies considerably between the flora of saline areas (halophytes) and the vast majority of plant species from ecosystems with low salt levels (glycophytes). Yet even halophytes will be affected, and will eventually die above a certain threshold of salinity. At damaging salt levels, glycophytes and halophytes face the same complications: insufficient osmotic adjustment resulting in reduced turgor, dehydration, stomatal closure and limited net photosynthesis, cytotoxic damage by $\mathrm{Na}^{+}$and $\mathrm{Cl}^{-}$ions in the cytoplasm, $\mathrm{K}^{+}, \mathrm{Ca}^{2+}$ and $\mathrm{Mg}^{2+}$ deficiency, and/or damage from ROS (Flowers and Colmer 2015). Salt tolerance can be defined as the ability to grow, even if more slowly, and produce harvestable yield in soils affected by 
salinity, and is usually expressed as the percentage of biomass/yield production in saline vs control conditions over a prolonged period of time (Munns et al. 2020a).

When exposed to salinity, plants display a large number of anatomical and physiological responses to tolerate the stressful conditions schematically represented in Figure 2. 


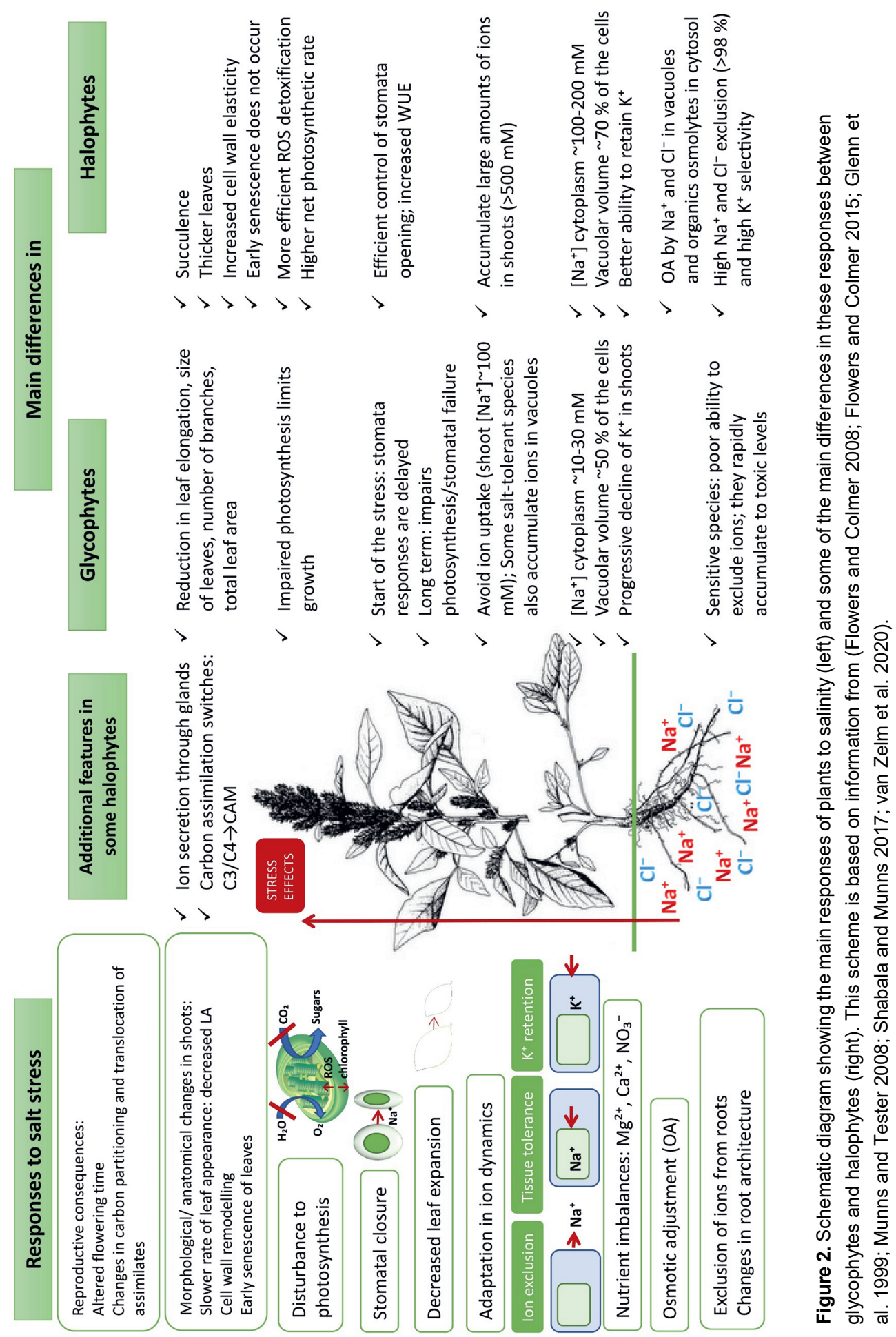


As most of the responses displayed in Figure 2 are common for glycophytes and halophytes, the high salt tolerance of halophytes is predominantly due to a greater robustness and efficiency of the employed mechanisms, rather than unique processes in these species (Isayenkov and Maathuis 2019). Knowledge of the mechanisms of salt tolerance is crucial for revealing the genetic and molecular basis of salt tolerance in plants with potential application for breeding of crops with enhanced ability to grow in saline soils. The essential salt tolerance mechanisms used by halophytes to withstand salt are summarized below (Flowers and Colmer 2008; Glenn et al. 1999; Flowers and Colmer 2015; Wungrampha et al. 2018):

- High ability to exclude $\mathrm{Na}^{+}$and $\mathrm{Cl}^{-}$from roots (from 90-99.6\%), and a highly selective $\mathrm{K}^{+}$- uptake system.

- Osmotic adjustment, relying more on the accumulation of inorganic ions than production and accumulation of organic ions. For example, in several species of Chenopodiaceae $\mathrm{Na}^{+}$and $\mathrm{Cl}^{-}$ions contribute about $65 \%$ of the total solute concentrations, while sugars contribute $1 \%$ (Flowers and Colmer 2008). Other species use $\mathrm{K}^{+}$and $\mathrm{SO}_{4}{ }^{2-}$ for osmotic adjustment (Ben Hamed et al. 2018).

- Compartmentalization of $\mathrm{Na}^{+}$and $\mathrm{Cl}^{-}$in the vacuole, coupled with the production of species-specific compatible solutes, (the most common ones: glycinebetaine, proline, inositol, pinitol, sorbitol and mannitol) in the cytoplasm.

- Accumulation of high $\mathrm{K}^{+}$concentrations in shoots (the ratio $\mathrm{Na}^{+} / \mathrm{K}^{+}$is normally between $0.5-1$ ).

- Accumulation of $\mathrm{Cl}^{-}$in the shoots for electrochemical balance of $\mathrm{Na}^{+}$and $\mathrm{K}^{+}$.

- Tight regulation of ion transport from roots to shoots.

- Increase in succulence (associated with an increase in cell size, decrease in surface area per tissue volume and higher water content per unit of leaf area).

- Control in stomatal closure is fast and efficient and it is not accompanied by loss of leaf water content. Together with morphological adaptation in leaves (e.g. succulence), it increases WUE in the plants and does not limit photosynthesis.

- Efficient neutralizing of reactive oxygen species (ROS).

- Maintenance of higher net photosynthetic rate by stabilizing the photosystems and protecting the photosynthetic machinery.

- Salt excretion through salt glands (recreto-halophytes). 
- Switch strategy of carbon assimilation (certain halophytes switch from C3 to CAM (e.g. Portulacaria afra, Mesembryanthemum crystallinum) or C4 to CAM (e.g. Portulaca oleracea)).

Considering the complexity and diversity of mechanisms that contribute to salt tolerance, breeding or engineering crops adapted to saline soils has proven to be a challenging task. In addition, investment in salt tolerance mechanisms normally come with trade-offs for crop productivity, which might make salt-tolerant varieties commercially uncompetitive (Gilliham et al. 2017; Wani et al. 2020; Yamaguchi and Blumwald 2005). Several approaches have been considered to develop agriculture in saline areas (Flowers 2004; Alqahtani et al. 2019):

- Improve farming practices to prevent secondary salinization

- Soil remediation to reduce the salt content in soils

- Incorporate new/ alternative salt-tolerant crops

- Exploit natural variation and use landraces or wild relatives of existing crops that might have improved salt tolerance to improve the salt tolerance of current crops

This thesis explores the third option mentioned above to face the challenge of salinity: introducing quinoa (Chenopodium quinoa), a novel, globally emerging salt tolerant crop, as an alternative to grow in areas affected by salinity where other crops are failing.

\section{The facultative halophyte crop quinoa}

Quinoa (Chenopodium quinoa Willd., $2 n=4 x=36$ ) is an allotetraploid, domesticated in the Andean region over 5000 years ago. Historical data indicate that it was probably domesticated by ancient civilizations in different areas including Peru, Chile and Bolivia (Bazile et al. 2013). Phylogenetic studies have tried to reconstruct the evolutionary history of quinoa and suggest that it originated from the hybridization of diploid progenitors (C. pallidicaule and C. suecicum), possibly involving intermediate tetraploid wild ancestors (C. berlandieri and C. hircicum) (Bazile 2015; Jarvis et al. 2017).

The genus Chenopodium includes about 150 species distributed around the world, but mostly concentrated in temperate and subtropical regions, especially in arid and/or saline environments (Bazile et al. 2013). It is a member of the family Amaranthaceae, and other economically relevant crops from this family are spinach (Spinacia oleracea) and sugar beet (Beta vulgaris). Amaranthaceae is closely related to the Chenopodiaceae family; in fact, strong similarities between them make 
their distinction difficult. Amaranthaceae include annuals, herbaceous perennials, shrubs and woody lianas distributed throughout tropical and subtropical latitudes, while the Chenopodiaceae family is mostly characterized by annuals and subshrubs predominantly found in arid and semiarid habitats (Kadereit et al. 2003). Remarkably, the Chenopodiaceae family has the largest number of halophytes of all angiosperms (Cheeseman 2015).

Quinoa displays broad genetic diversity that allows it to adapt to different ecological environments. Worldwide, more than 16000 accessions of quinoa and its wild relatives are conserved in 59 genebanks distributed over 30 countries. Most of the germplasm is conserved by Andean research institutes (Rojas 2015).The diversity of quinoa can be classified in five ecotypes that reflect the distribution of the species from its center of origin around Lake Titicaca. These ecotypes are associated with sub-centers of diversity and are highly adapted to specific environments (Bazile et al. 2016a). The geographical distribution of quinoa ecotypes is depicted in Figure 3.

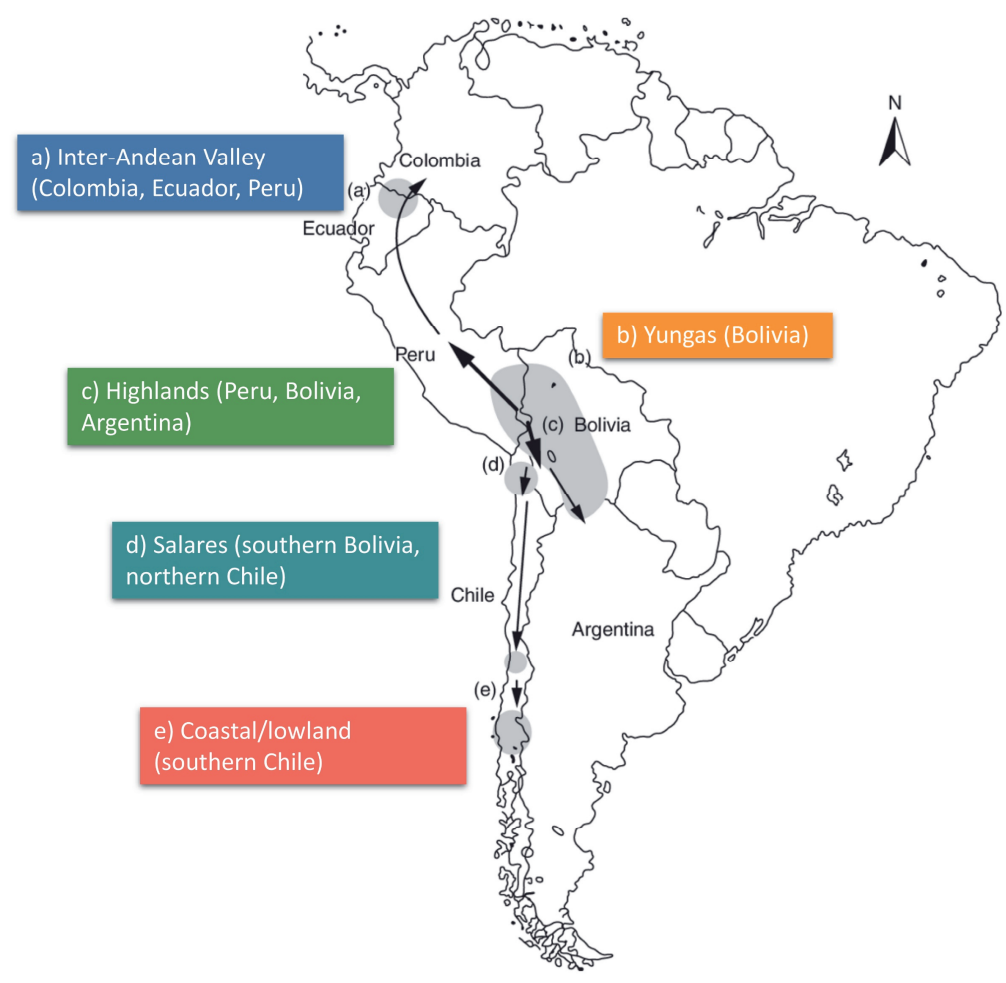

Figure 3. Geographical distribution of the five ecotypes of quinoa throughout the Andean Region (adapted from (Bazile et al. 2013)). 
For centuries quinoa cultivation was limited to small rural (mostly indigenous) communities in the Andes. From the second half of the $20^{\text {th }}$ century it became an internationally emerging crop; the number of countries growing quinoa has risen from 8 in the 80 s to 95 in 2015, many of them actively performing field trials before launching field production in the near future (Bazile et al. 2016a). The main quinoa producers in the world are Bolivia and Peru. These two countries grow up to $80 \%$ of the world total production, while the remaining $15-20 \%$ is produced by Ecuador, USA, China, Chile, Argentina, France and Canada (Bazile et al. 2016a). Figure 4 depicts the growth in quinoa global production during the last two decades. The global expansion of quinoa can be divided in two phases. First, importer countries showed interest in adapting the crop to their environments and develop national breeding programs; this is the case for United States of America, Canada, France, United Kingdom, Denmark and The Netherlands. The second phase involves a strong interest of using quinoa as an alternative crop in response to climate change and salinization of agricultural land. This is the case for India, Vietnam, Pakistan, China, Australia and several countries around the Mediterranean Sea and in Northern Africa (Bazile 2015).

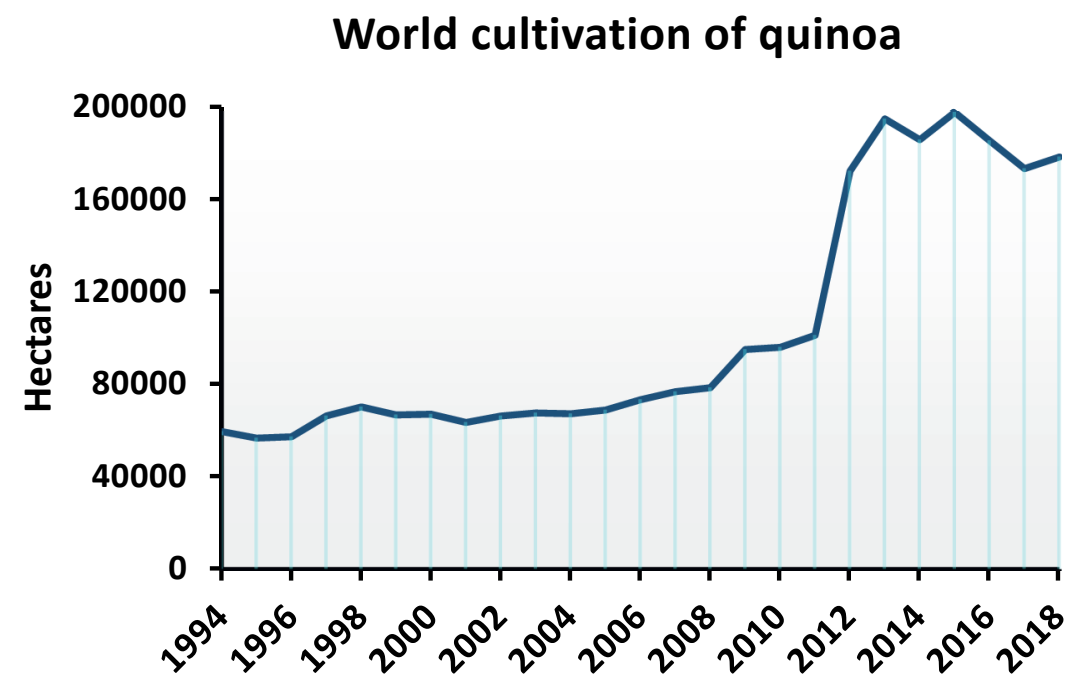

Figure 4. Global annual production of quinoa from 1994-2018. Data from (FAO 2020). 
Several factors have contributed to the rapid expansion of quinoa. It is a highly nutritious crop with a balanced composition of protein, minerals, fiber, antioxidants and vitamins. Moreover, it does not contain gluten and is therefore suitable for celiac patients. The main nutritive qualities of quinoa are listed below (Angeli et al. 2020; Nowak et al. 2016; Kozioł 1992):

- Higher protein content than other cereals (rice, barley, corn, rye, sorghum); similar to wheat. The protein content of quinoa seeds ranges from 11-19\% of the total dry matter content (DMC).

- High quality protein: contains the nine essential amino acids in a balanced pattern. It is particularly rich in lysine (5.4\% total protein) and histidine (2.9 $\%$ total protein).

- The total dietary fiber content ranges from 7-13\% DMC, up to $30 \%$ of which is soluble.

- Quinoa oil is rich in essential fatty acids (88\% of the total fat content); the main ones are oleic (19.7-29.5\%), linoleic (49-56.5\%) and linolenic acid (8.7-11.7\%).

- Contains more calcium, iron, magnesium, copper, manganese and chloride than cereals.

- $\quad$ Rich in vitamins, especially riboflavin (B2) and a-tocopherol (E).

- Quinoa leaves are rich in phenolic components, beneficial to human health due to their antioxidative potential. The most abundant are ferulic, sinapinic and gallic acids, kaempferol, isorhamnetic and rutin.

- The most important anti-nutritional factor in quinoa is the presence of saponins, secondary metabolites present in the seed coat that confer a bitter taste to the seeds. The content of saponins in the seeds varies from 20-40 $\mathrm{mg} \mathrm{g}^{-1}$ (sweet genotypes) to as high as $470 \mathrm{mg} \mathrm{g}^{-1}$ (bitter genotypes).

The nutritional composition of quinoa is superior to most of the cereals, for comparison, some of the nutritional properties of quinoa, rice and wheat are compared in Table 1. 
Table 1. Comparison of some nutritional parameters from quinoa, wheat and rice. Data from (U.S. Department of Agriculture 2020).

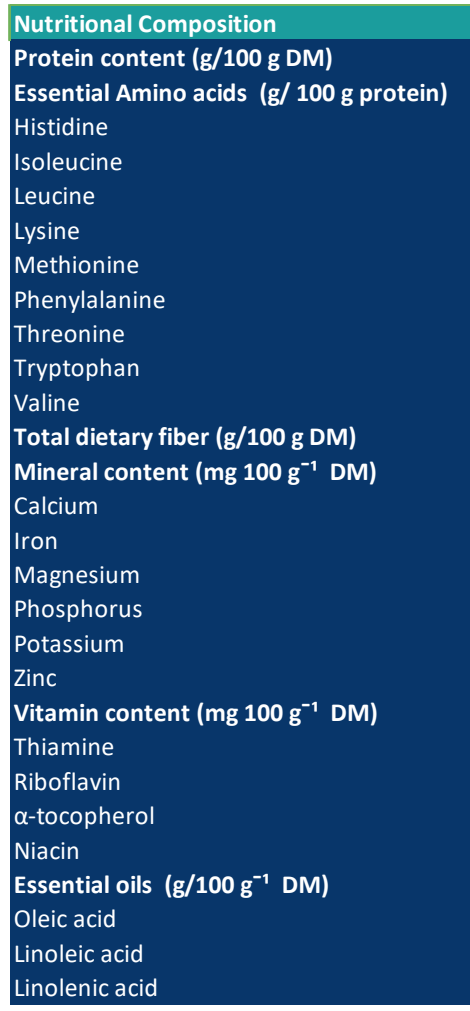

Nutritional Composition

Protein content (g/100 g DM)

Essential Amino acids (g/ $100 \mathrm{~g}$ protein)

Histidine

Isoleucine

Leucine

Methionine

Valine

Total dietary fiber ( $/$ /100 g DM)

(mg $100 \mathrm{~g}^{-1}$ DM)

Iron

Magnesium

Zinc

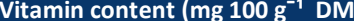

amine

Niacin

(g/100 $\left.\mathrm{g}^{-1} \mathrm{DM}\right)$

Linoleic acid

Linolenic acid
Quinoa (USDA ID 168874) Wheat (USDA ID 169721) Rice (USDA ID 169756)

13.68

7.13

0.407

0.504

0.84

0.766

0.309

0.593

0.421

0.167

0.594

7

47

4.57

197

457

563

3.1

0.36

0.318

2.44

1.52

1.42

2.977

0.26 $\begin{array}{ll}0.322 & 0.168\end{array}$

$0.533 \quad 0.308$

$0.934 \quad 0.589$

$0.303 \quad 0.258$

$0.221 \quad 0.168$

$0.681 \quad 0.381$

$0.366 \quad 0.255$

$0.176 \quad 0.083$

$0.594 \quad 0.435$

$12.7 \quad 1.3$

$34 \quad 28$

$3.5 \quad 0.8$

$144 \quad 25$

$508 \quad 115$

$431 \quad 115$

$\begin{array}{ll}4.2 & 1.09\end{array}$

$\begin{array}{ll}0.419 & 0.07\end{array}$

$0.121 \quad 0.049$

$1.01 \quad 0.11$

$\begin{array}{ll}6.738 & 1.6\end{array}$

$0.335-0.203$

$0.93 \quad 0.146$

$0.048 \quad 0.031$

In addition to its high nutritional value, another important driver for the rapid worldwide expansion of quinoa is its resilience to grow in suboptimal environments. It has survived the rough environments from the Andes for many thousands of years and is able to tolerate different relative humidity conditions (40-88 \%), large range of altitudes (from sea level up to 4000 m.a.s.I.), a wide range of temperatures (-8 to $38^{\circ} \mathrm{C}$ ), hail, frost, drought and soil salinity (Gomez-Pando et al. 2019).

Quinoa is considered a facultative halophyte, meaning that it grows and thrives under non-saline or low-saline conditions, it grows well with no or limited yield loss up to a threshold of salinity $(100-200 \mathrm{mM} \mathrm{NaCl})$ and is able to survive in soils with salt levels as high as those of seawater ( $>400 \mathrm{mM} \mathrm{NaCl}$ ); quinoa is considered one of the most salt tolerant crops (Hinojosa et al. 2018). In the Andean Region, it can grow and is cultivated in areas characterized by severe salinity, such as the salt flats in Bolivia (Ruiz et al. 2016b). Successful field trials in areas affected by salinity in Vietnam, Iran, United Arab Emirates, etc. (Choukr-Allah et al. 2016; Nguyen et al. 
2020; Razzaghi et al. 2020) further demonstrate the potential of this crop for cultivation on marginal lands.

The germination of quinoa is mostly delayed under salinity levels of up to $400 \mathrm{mM} \mathrm{NaCl}$, while germination rate is not affected for most of the assessed genotypes (Adolf et al. 2013). Differences have been reported for the impact of salinity on quinoa seed yield; for some genotypes even higher yields were reported under $100-200 \mathrm{mM} \mathrm{NaCl}$ salinity than under non-saline conditions; in others, yield penalties were reported at salinity level above $80 \mathrm{mM} \mathrm{NaCl}, 50 \%$ yield reduction at $250 \mathrm{mM} \mathrm{NaCl}$, and survival of plants at up to $500 \mathrm{mM} \mathrm{NaCl}$ (Adolf et al. 2013; Hirich et al. 2014; Razzaghi et al. 2015). Efforts are being made to identify predictors of seed yield during the growth of the crop-neither stomatal conductance, inflorescence size or plant height could be successfully used with this purpose (Adolf et al. 2013). Several studies have tried to assess the potential mechanisms contributing to quinoa salt tolerance; key traits reported are efficient control of xylem $\mathrm{Na}^{+}$loading and sequestration into vacuoles, high $\mathrm{ROS}$ tolerance, good $\mathrm{K}^{+}$retention, and an efficient control of stomatal opening (Adolf et al. 2013; Hariadi et al. 2011; Orsini et al. 2011; Razzaghi et al. 2015). It has been recognized that high genetic variation for salt tolerance can be found in quinoa, and only a small part of the whole diversity of this species has been tested (Ruiz et al. 2016b). In addition, most of the studies evaluate the response to salinity after only a few weeks of stress, while under prolonged salinity the stress in the plant is likely to build up. The contribution of most of the salt tolerance traits to the yield of quinoa under saline conditions is largely unknown, and the genetic determinants of these traits remain to be established.

\section{Understanding quinoa's ability to cope with salinity: outline of this thesis}

This thesis uses physiological, biochemical and genetic approaches to provide novel insights into the remarkable salt tolerance of quinoa. We explore the facultative halophytic nature of this species; by comparing several responses of the plants growing without salt, and salt concentrations considered "moderate" and "high" for quinoa we try to differentiate the physiological adaptations that favour its growth under salinity from the ones that enable its survival under extreme conditions. We approach salinity as a dynamic process during the development of the crop, so stress responses were monitored throughout the growing cycle of the plants starting with young plantlets, taking into account whole-plant as well as tissue-specific changes. We compare salinity-induced adaptations between genotypes that point to different strategies being used depending on the stress severity and duration, with 
different consequences for growth and yield. We consider genetic diversity as the most valuable asset for salt tolerance breeding, so we studied salinity responses of several commercial varieties, mapping populations as well as exotic germplasm. Figure 5 gives an overview of the organisation of this thesis.

In Chapter 2, we evaluate responses of quinoa varieties to long-term high salinity with respect to growth (RGR components, photosynthetic efficiency, ion dynamics) and ion homeostasis. By analysing ion concentrations in root, stem and leaf tissue over time we demonstrate that the uptake, transport and distribution of ions during prolonged salt stress is dynamic in quinoa: it changes with time and developmental stage and differs between varieties.

High salinity changes the anatomy of the plants. Changes in turgor and cell growth are strongly related to changes in cell walls; so, in Chapter 3 we compare the cell wall composition of leaves and stems from plants growing under control and stress conditions and reflect on how these changes influence the plasticity of the cells to adapt to salinity.

In Chapter 4 we incorporate the Plantarray phenotyping platform ${ }^{\circledR}$ to monitor the effects of salinity on growth in quinoa with high temporal resolution. We obtained detailed information on adaptations in transpiration, water use efficiency and plant water relations that are part of the strategies used by quinoa genotypes to cope with salinity. We reflect on the advantage provided by this type of automated functional phenotyping to understand how plants use resources (light, water, nutrients) and how this can be used to construct a simple growth model for quinoa under salinity.

In the first three experimental chapters, salt tolerance mechanisms were evaluated in spaced plants in greenhouse conditions. In Chapter 5, we explore agronomical, physiological and biochemical responses of commercial varieties to a salt stress of $250 \mathrm{mM} \mathrm{NaCl}$ under more agronomical conditions that resemble field cultivation. In addition, we evaluate two mapping populations to explore the genetics underlying salt tolerance responses; genetic mapping was used to identify genetic regions, that can be further explored for potential candidate genes and alleles and implemented in breeding programs for salt tolerance and agronomical traits of interest in quinoa.

Genetic diversity is the basis for crop improvement. Quinoa has broad genetic diversity that needs to be further explored and exploited. In Chapter 6 we evaluate the genetic diversity for salt tolerance of 22 genotypes of the Inter-Andean valley ecotype from Ecuador. These genotypes are good representatives of the 
quinoa that is currently cultivated in Ecuador. We explore the potential of this material as new sources for salt tolerance traits and other agronomical aspects of interest.

The results presented in the different chapters are discussed in Chapter 7. I try to integrate all the mechanisms explored throughout the thesis into an ideotype of a salt tolerant quinoa. I reflect on the usefulness of quinoa as a model for salt tolerance research and explore which mechanisms might be possibly incorporated in other crops. Finally, I discuss the future perspectives of quinoa, and the steps that have been taken and need to be taken in the future to consolidate this crop as an international food staple commodity. 

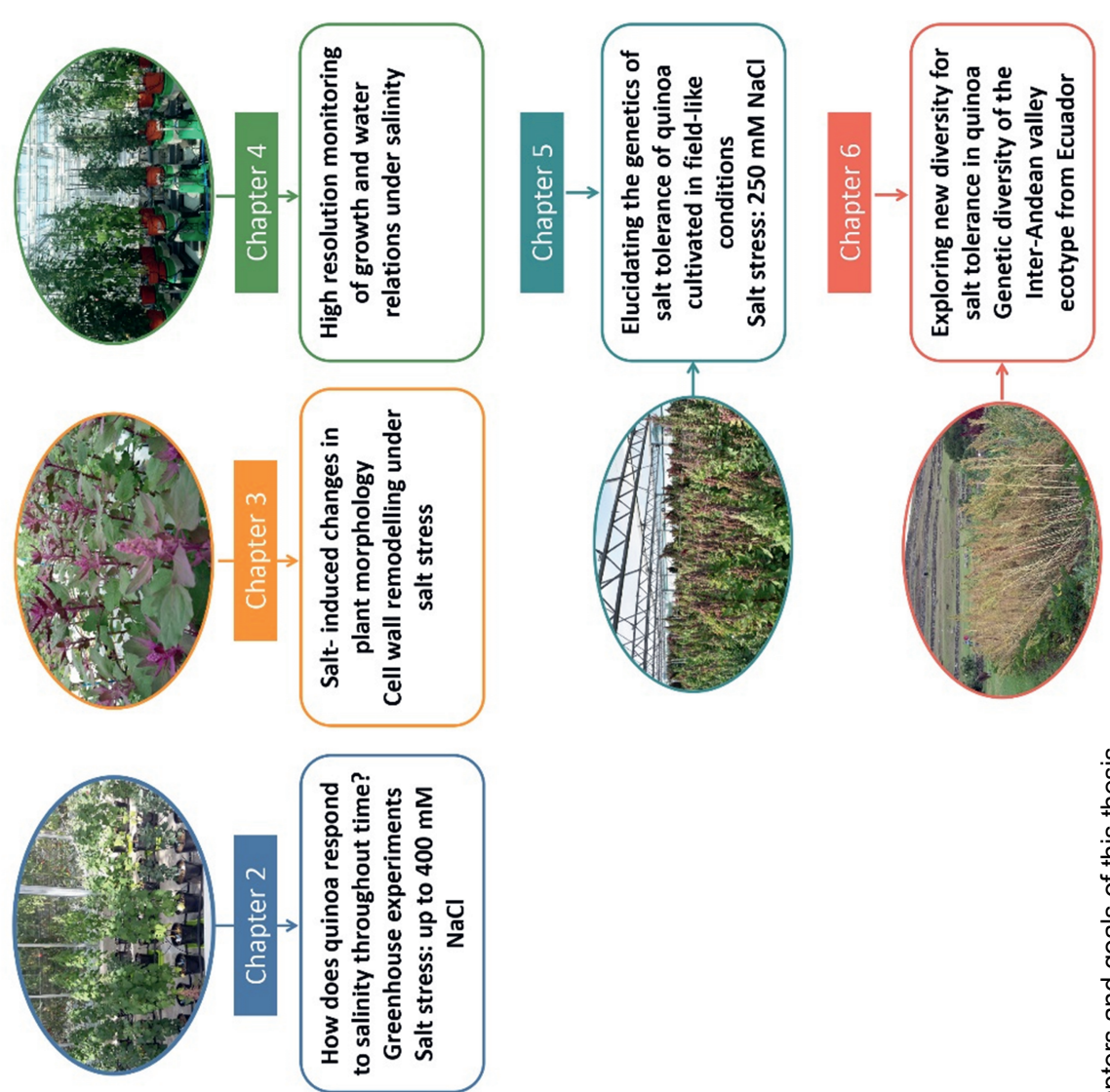

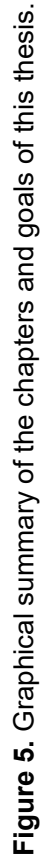




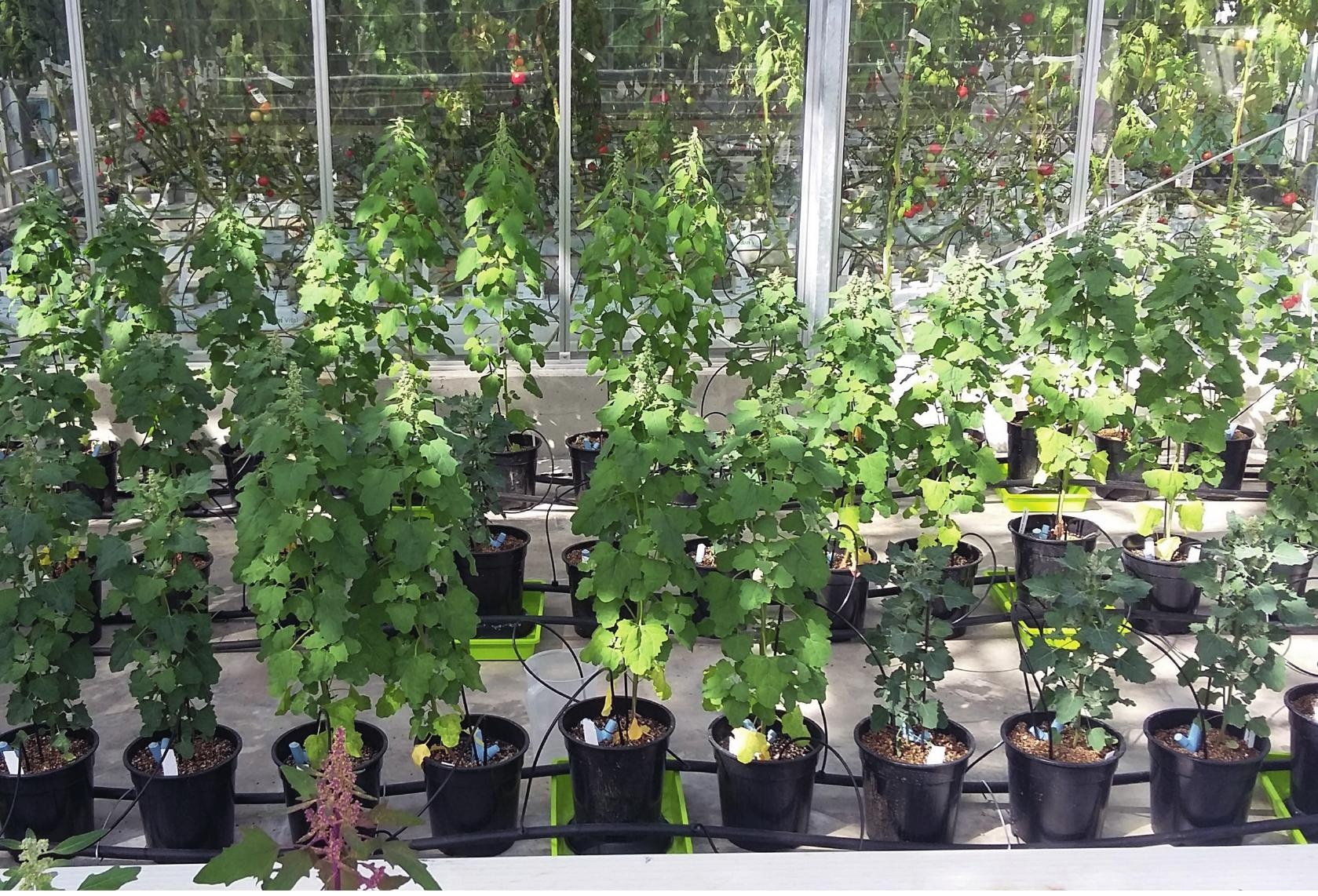

\section{Chapter 2 Differential responses to salt stress in ion dynamics, growth and seed yield of European quinoa varieties}

Viviana Jaramillo Roman, Leonardus A. den Toom, Carlos Castro Gamiz, Niels van der Pijl, Richard G.F. Visser, Eibertus N. van Loo, C. Gerard van der Linden

This chapter has been published as: Jaramillo Roman, V., den Toom, L.A., Castro Gamiz, C., van der Pijl, N., Visser, R.G.F., van Loo, E.N., van der Linden, C.G. (2020). Differential responses to salt stress in ion dynamics, growth and seed yield of European quinoa varieties. Environmental and Experimental Botany. 177:104146. doi: https://doi.org/10.1016/j.envexpbot.2020.104146 
Quinoa is a nutritious seed crop with a great potential to grow in saline soils. Here, we studied ion concentrations in quinoa tissues throughout the life cycle of the plant, and linked ion dynamics to responses in growth parameters, seed yield and efficiency of photosynthesis under salinity $(0-400 \mathrm{mM} \mathrm{NaCl})$. Ion dynamics changed from high ion exclusion (>99\%, root contents lower than root medium and low accumulation of ions in the leaves) before flowering, to a build-up of ions during seed filling. This indicates a change in strategy in maintaining the necessary gradient of water potential from the root medium to the leaves. $\mathrm{K}^{+}$concentrations in leaves also increased by more than $100 \%$ in response to prolonged severe salt stress, which may point to a role of this ion in leaf osmotic adjustment. Accumulation of ions in epidermal bladder cells did not contribute substantially to $\mathrm{Na}^{+}$-exclusion as it was less than $6 \%$ of the total $\mathrm{Na}^{+}$taken up in leaves. Growth under salt stress was mostly impaired by anatomical adaptations (reduced SLA), while initial light use efficiency (Fv/Fm) and NAR were not affected. The variety Pasto showed a "survival strategy" to high salinity with higher ion exclusion and a higher reduction in transpiration than the other varieties, at the expense of lower biomass and seed yield. 


\section{Introduction}

Soil salinity is a major abiotic stress that seriously threatens plant growth and food security (Roy et al. 2011). In the coming decades, salt-affected agricultural areas will expand as a consequence of both climate change and poor land management (Daliakopoulos et al. 2016). Remediation of salt-affected land is necessary but will take years before standard food crops can be grown again, so the development of resilient crops that can survive and be productive on these conditions should complement remediation of saline soils.

Halophytes are plant species that are naturally well adapted to high salinity, and can survive, grow and reproduce under extreme saline conditions (Flowers and Colmer 2008). However, most halophytes are of little interest for agriculture as their yields are too low or their biomass unsuitable as food or feed (Shabala 2013). One of a few exceptions is quinoa (Chenopodium quinoa). Quinoa is considered one of the most salt tolerant crop species, even more tolerant than barley or wheat (Murphy and Matanguihan 2015). Originating from the Andean Altiplano, quinoa is adapted to a broad range of ecosystems and abiotic stresses including saline soils, drought and frost (Zurita-Silva et al. 2014). Quinoa grows optimally under low or no salinity, but it can still produce seeds at soil salt levels that equal or even surpass those of seawater, and is therefore classified as a facultative halophyte (Mishra and Tanna 2017). The ability to produce relatively high yields on saline soils where other crops are highly affected or failing justifies the designation of quinoa as an essential crop to ensure food security (Zurita-Silva et al. 2014).

The highest reported soil electrical conductivity (EC) level at which quinoa was able to survive was $51.5 \mathrm{dS} / \mathrm{m}$, while $50 \%$ reduction in yield was found at an EC of $25 \mathrm{dS} / \mathrm{m}$ (Razzaghi et al. 2015). Some studies claim that optimal growth and performance of quinoa can be achieved between 10 and $20 \mathrm{dS} / \mathrm{m}$ (Adolf et al. 2013; Hariadi et al. 2011; Jacobsen et al. 2003), while others state that quinoa plants start to be affected at salinity levels of 8-10 dS/m (Geissler et al. 2015; Hirich et al. 2014). These differences point to the existence of a rich pool of genetic resources that can be used for breeding quinoa varieties with improved yield under high salinity. In addition, the remarkable resilience of quinoa may also provide new insights into salt tolerance mechanisms that can be extended as breeding targets for other species.

Salt tolerance is a complex trait that requires a coordinated response of the plant to withstand the osmotic and ionic stress that salinity imposes on the plant. Plant species have a variety of responses to overcome both. Salinity decreases the osmotic potential of the soil, which leads to decreased turgor pressure in root cells 
and consequently water loss (Julkowska and Testerink 2015). To avoid water loss, a first response of the plant is to close stomata and reduce transpiration at the cost of lower cell extension rate and growth. The maintenance of turgor is also facilitated by decreasing the osmotic potential in the roots, which is achieved by increasing the concentration of osmolytes in tissues. Osmotic adjustment is an essential plant response to salt stress, and can be achieved by the synthesis of organic compounds, or the accumulation of $\mathrm{Na}^{+}$and $\mathrm{Cl}^{-}$in a cost-effective manner (Munns et al. 2016). In addition to the challenge of transporting water under salt stress, the plant has to deal with the salt ions $\left(\mathrm{Na}^{+}\right.$and $\left.\mathrm{Cl}^{-}\right)$that are taken up and that are toxic at high concentrations. Several strategies have been described with the main goal of keeping ion concentrations low in the cytosol, particularly in the mesophyll cells in the leaves. Ions can be excluded, or secreted, from root tissues back to the root medium, or retrieved from xylem parenchyma cells by specific and well-studied ion transporters, (like SOS1 and HKT type 1) (Møller and Tester 2007). A recent review examines the implications of keeping ion concentrations in shoots of plants low (Munns et al. 2020b). Maintaining low levels of ions in the shoot over a longer period of time requires a high level of $\mathrm{Na}^{+}$exclusion in plants (and $\mathrm{Cl}^{-}$exclusion to a lesser extent). The longer a plant is exposed to high salinity, the more challenging it will be to maintain low shoot ion levels. However, only a few studies have examined dynamics of ion accumulation throughout plant development (Ashraf and Khanum 1997; Sairam et al. 2002), and whether the high level of exclusion is sustained through the life cycle of plants remains unanswered.

This paper examines the dynamics of ion homeostasis from young plants until seed maturation in different tissues of quinoa plants. Several reports have studied physiological traits that might explain the high salt tolerance in quinoa and showed broad genetic diversity in the extent of exclusion of ions (mainly $\mathrm{Na}^{+}$) from shoots (Hinojosa et al. 2018), but most of these studies focused on rather young plants and relatively short duration of salt stress (Adolf et al. 2012; Hariadi et al. 2011; Shabala et al. 2013; Ruiz-Carrasco et al. 2011). We evaluated a set of commercial varieties at several degrees of salinity severity throughout the crop cycle to identify potential strategies of quinoa to adapt to prolonged exposure to salt stress. The variety of responses of quinoa cultivars described here demonstrate that quinoa qualifies as a model crop for studying halophytic salinity tolerance mechanisms. 


\section{Materials and Methods}

\subsection{Plant Materials}

Four European non-bitter (sweet) quinoa varieties were used in the different experiments described below: Atlas, Jessie, Pasto, a line (selRiobamba) selected from Riobamba (Riobamba has still some residual heterozygosity) and one darkseeded, bitter variety (Red Carina). The varieties were bred at Plant Breeding, Wageningen Research (The Netherlands) and AbbottAgra (France) and are adapted to the Western European climate and photoperiod.

\subsection{Experimental conditions and treatments}

Three experiments were carried out in three consecutive years (2015-2017). All the experiments were performed using spaced plants in $3 \mathrm{~L}$ pots. The plants were irrigated with half-concentrated Hoagland's nutrients solution. Salt treatments started five weeks after sowing, when plants had four fully developed pairs of leaves. Salt was applied by incremental increases of $75 \mathrm{mM}$ per day until the desired salt concentration was reached. Salt concentrations were monitored regularly by measuring the electrical conductivity in the leakage from the pots with a conductivity meter (Profline Cond 315i, Xylem Analytics, Germany). All the experiments were conducted at the Unifarm greenhouse facilities of Wageningen University \& Research, The Netherlands between April and September under natural photoperiodic (long day) conditions. The greenhouse air humidity was set to a minimum of $80 \%$. When the incoming shortwave radiation was below $200 \mathrm{Wm}^{-2}$, additional lighting was supplied $\left(100 \mathrm{Wm}^{-2}\right)$. Light irradiance, air temperature, water content and electrical conductivity $(E C)$ in the pots were monitored via wireless sensors (Flower Power ${ }^{\mathrm{TM}}$ ).

The first experiment (2015) aimed to evaluate the general performance of European sweet quinoa growing at different levels of soil salinity. The varieties Atlas, Jessie, Pasto and selRiobamba were grown at four different levels of salinity: 0, 100, 200 and $300 \mathrm{mM} \mathrm{NaCl}$. The experiment was done in a screenhouse using vermiculite as substrate, and the pots were drained with saline solution frequently to maintain a stable level of salinity in the pots. Ten plants per variety were used for each treatment. Half of the replicates were harvested ten weeks after sowing, during the vegetative phase growth of the plants. The other half was harvested at seed maturity (20 weeks after sowing).

The second experiment (2016) included the same varieties used in Experiment 1 , plus the dark bitter variety Red Carina, grown at high salinity levels: 300 and 400 
$\mathrm{mM} \mathrm{NaCl}$. Eight plants per variety were used for each treatment. Half of the replicates were harvested at the onset of flowering (11 weeks after sowing). The other half was harvested at seed maturity (20-24 weeks after sowing). The experiment was done in the greenhouse using fine vermiculite (size 1) as substrate.

The third experiment (2017) was a time series experiment using the most contrasting varieties in terms of agronomical and salt tolerance related traits from Experiments 1 and 2 (Jessie, Pasto and selRiobamba) and a severe salt stress of $400 \mathrm{mM} \mathrm{NaCl}$. Three replicates per variety were harvested at four different time points during the growing season: 9, 12, 16 and 20 weeks after sowing. As we encountered draining problems with fine vermiculite as substrate resulting in salt accumulation in the pots in the second experiment, we switched to course vermiculite (size 3 ) in this experiment.

\subsection{Assessment of growth traits}

Plant height was measured weekly. Plant developmental stages were scored weekly according to a cardinal scale adapted from Masterbroek et al. (2002) (Table 1). During each destructive harvest, the biomass of the plants was separated into above-ground biomass (stems, leaves, heads) and roots. Leaves were removed from the plant and separated into young leaves (one-third upper part of the plant) and old leaves (two-third lower part of the plant). Fresh weights of leaves, stems, heads and roots were recorded, and leaf area was measured using a leaf area meter (Li-3000 Area Meter, Li-Cor, Lincoln, NE, USA). Dry weights were determined after drying leaves, roots and stems in a forced-air oven at $70^{\circ} \mathrm{C}$, (seeds at $35^{\circ} \mathrm{C}$ ), until samples reached stable weights. During the vegetative growth of the plants (from the transplanting date: three weeks after sowing, until the first destructive harvest: nine weeks after sowing) relative growth rate $\left(R G R, \mathrm{~d}^{-1}\right)$ and its specific components were calculated based on the linear relation $R G R=L W R \times S L A \times N A R$. NAR is the net assimilation rate $\left(\mathrm{g} \mathrm{m}^{-2}\right.$ day $\left.^{-1}\right)$, LWR is leaf weight ratio $\left(\mathrm{g} \mathrm{g}^{-1}\right)$, and SLA is the specific leaf area $\left(\mathrm{m}^{2} \mathrm{~kg}^{-1}\right)$. SLA was calculated as the amount of leaf area per unit of leaf dry weight, LWR as the leaf fraction of the total dry plant biomass, and RGR as the natural logarithm of the relative increase in plant biomass over the mentioned period of time: $R G R=\ln \left(W_{2} / W_{1}\right) /\left(t_{2}-t_{1}\right)$ (Lambers and Poorter 1992). After physiological ripening, seed yield was measured as dry seed weight per plant, thousand seed weight (TSW) was recorded using a seed counter (Contador, Pfeuffer $\mathrm{GmbH}$, Jefferson, OR, USA) and harvest index $(\mathrm{HI})$ was calculated as the ratio of dry seed weight and dry aboveground biomass. The salt tolerance index (STI) was 
calculated as the ratio of dry biomass (above-ground biomass or seed yield) of salttreated plants and the dry biomass of control $(0 \mathrm{mM} \mathrm{NaCl})$ plants.

Table 1. Plant development stages in quinoa. Adapted from (Mastebroek et al. 2002).

\begin{tabular}{l|l}
\hline Stage & Description \\
\hline F1 & Flower buds just visible \\
F2 & Flower buds $1.0 \mathrm{~cm}$ \\
F3 & First glomeruli show anthers \\
F4 & $50 \%$ glomeruli show anthers \\
F5 & Wilted anthers \\
F6 & Seeds watery ripe/ panicle green \\
F7 & Seeds milky ripe/ panicle green \\
F8 & Seeds dough ripe/ beginning panicle coloration \\
F9 & Seeds physiological ripe / panicle fully coloured \\
\hline
\end{tabular}

\subsection{Assessment of physiological traits}

Several physiological traits were measured during the growing season. Stomatal conductance ( $g s$ ) was measured in the second fully developed non-shadowed leaf using a portable leaf porometer (Decagon Devices Inc., WA, Australia) throughout the growth cycle between 10:00-12:00 hours on a sunny day, unless specified otherwise. Leaf chlorophyll content was measured using a SPAD 502 meter (Minolta, Osaka, Japan) on the second fully developed leaf. The maximum photochemical efficiency of photosystem II after dark adaptation ( $\mathrm{Fv} / \mathrm{Fm}$ ) was measured on the same leaf, between 10:00- 12:00 using an OS/30P portable fluorometer (OpticsScience Inc., USA). Relative water content was calculated at the onset of flowering as $R W C=\frac{(F W-D W)}{(T W-D W)} * 100 \%$, where TW is the turgid weight, FW is the fresh weight and DW is the dry weight of an entire single young leaf. Turgid weight was determined after the leaf was imbibed in ultrapure water (Milli- $\mathrm{Q}^{\circledR}$ ) in the dark for $12 \mathrm{~h}$.

\subsection{Ion content measurements}

The ion contents in leaves, stems, roots and bladder cells were measured using Ion Chromatography (IC) system 850 Professional (Metrohm Switzerland). For this purpose, oven-dried tissues were ground to fine powder using a hammer mill with 1 $\mathrm{mm}$ sieve. Twenty-five $\mathrm{mg}$ per sample was turned into ash in a furnace at $550^{\circ} \mathrm{C}$ for $5 \mathrm{~h}$. Ten $\mathrm{ml}$ of Milli-Q ${ }^{\circledR}$ water was added to the ashes and these were shaken for 15 min at $5000 \mathrm{rpm}$ at $100^{\circ} \mathrm{C}$. Prior to injection onto the IC system, samples of leaves, stems and roots were diluted 400 times with Milli- $Q^{\circledR}$. Nitrate was also measured 
using Ion Chromatography but the samples were prepared differently. Forty mg of grinded dry leaves was weighed in a glass screw cap tube. Five $\mathrm{ml}$ of Milli- $\mathrm{Q}^{\circledR}$ was added to the sample and this was mixed by vortexing for $5 \mathrm{~min}$. After shaking, the samples were heated in an ultrasonic bath for $30 \mathrm{~min}$ at $80^{\circ} \mathrm{C}$. The samples were transferred to a thermomixer and incubated for $1 \mathrm{~h}$ at $5000 \mathrm{rpm}$ at $100{ }^{\circ} \mathrm{C}$. After cooling down, samples were centrifuged for $5 \mathrm{~min}$ at $4200 \mathrm{rpm}$ and diluted 50 times previous the injection to the IC column. Ion contents were calculated as the amount of ions per unit of dry weight ( $\mathrm{mg}$ ion $\mathrm{g}^{-1}$ dry mass) and the ion concentrations were estimated based on the water content of the tissue. The ratio $\mathrm{K}^{+} / \mathrm{Na}^{+}$was calculated based on $\mathrm{mg} \mathrm{K}^{+} / \mathrm{mg} \mathrm{Na}^{+}$content.

\subsection{Characterization of epidermal bladder cells (EBCs)}

A dedicated experiment was conducted in order to obtain enough epidermal bladder cells to evaluate their potential function as deposits of salt ions during salt stress in quinoa. Plants of the cultivar Pasto were grown either in control conditions or with a salt concentration of $250 \mathrm{mM} \mathrm{NaCl}$. After eight weeks of treatment, 200 leaves were collected from control and treated plants. EBCs were brushed from the abaxial and adaxial sides of half of the leaves. The fresh weight of the 100 intact leaves, 100 leaves after removing the bladders, and the brushed bladders was recorded and the leaf area was measured as described before. The ion content in the leaves and in the EBCs was measured as previously described, but the EBCs were reduced to ashes and weighed without the grinding step during the sample preparation. The total biomass of both leaves and EBCs was decomposed as follows: fresh weight is the total biomass; $F W=W$ ash $+W$ water $+W$ organic matter; $\mathrm{W}$ water $=\mathrm{FW}-\mathrm{DW}$; and $\mathrm{W}$ organic matter $=\mathrm{DW}-\mathrm{W}$ ash .

\subsection{Statistical analysis}

General analyses of variance (ANOVA) were performed to determine the significance of genotypic differences, salt treatment differences and their interactions $(p<0.05)$. The analyses were performed following a standard procedure for a linear mixed model, for which genotype and salt treatment were considered fixed effects and blocks random effects. The above-mentioned model was: $y_{i j k}=\mu+b_{k}+\alpha_{i}+$ $d_{i k}+\beta_{j}+\alpha \beta_{i j}+e_{i j k}$, were $y_{i j k}$ is the response variable, $\mu$ is the grand mean, $\alpha_{i}$ is the salt treatment effect, $\beta_{j}$ is the genotype effect, $\alpha \beta_{i j}$ is the genotype-by-salt interaction effect, $b_{k}$ and $d_{i k}$ are the block effects and $e_{i j k}$ is the residual error. Multiple comparison analyses were performed using Fisher's protected least significant difference (LSD) test on genotype means. All statistical analyses were 
performed using the software Genstat $19^{\text {th }}$ Edition (VSN International Hemel Hempstead, UK).

\section{Results}

\subsection{Experiment 1: Full plant cycle response of European sweet quinoa to a wide range of salinity levels}

\subsubsection{Overall performance}

Four sweet quinoa genotypes: Atlas, Jessie, Pasto and selRiobamba were grown at four salt concentrations: $0,100,200$, and $300 \mathrm{mM} \mathrm{NaCl}$. Plant biomass was decreased significantly already at the time of the first destructive harvest (11 weeks after sowing, 6 weeks of treatment) when plants started to flower (Figure 1A), but the mean reduction was only $5 \%$ at $100 \mathrm{mM} \mathrm{NaCl}$, while it reached $43 \%$ at $300 \mathrm{mM}$ $\mathrm{NaCl}$. The averaged salt tolerance index (STI) at the onset of the flowering was 0.96 at $100 \mathrm{mM}, 0.79$ at $200 \mathrm{mM}$ and 0.62 at $300 \mathrm{mM} \mathrm{NaCl}$ (Figure 1B). Interestingly, until this stage, Jessie and selRiobamba had higher aboveground biomass at $100 \mathrm{mM}$ $\mathrm{NaCl}$ than under control conditions.

The effect of salt on seed yield was examined at the end of the growing cycle (Figure 1C). There was significant variation between genotypes and treatments, but not for the interaction between both. SelRiobamba was the variety with the highest yield, followed by Atlas and Pasto, and Jessie with the lowest yield. The average salt-induced seed yield reduction for the cultivars was $29 \%$ at $100 \mathrm{mM}, 57 \%$ at 200 $\mathrm{mM}$ and $65 \%$ at $300 \mathrm{mM} \mathrm{NaCl}$. SelRiobamba and Pasto had the lowest yield reduction ( $25 \%$ at $100 \mathrm{mM} \mathrm{NaCl}$ salinity). At the most severe salinity treatment $(300 \mathrm{mM} \mathrm{NaCl})$ selRiobamba remained the least affected variety (60\% reduction) but Pasto was more affected than Jessie (68\%). Significant genotypic variation ( $p$ $<0.05$ ) was detected for Harvest Index (HI) (Figure 1D), but surprisingly, HI was not significantly affected by the salt treatment. On average, the harvest index was reduced by only $4 \%$ at $100 \mathrm{mM}$ and by $20 \%$ at $300 \mathrm{mM} \mathrm{NaCl}$. In fact, it was the least affected parameter by salinity, which reflects the halophytic property of quinoa to still be able to allocate carbon to seeds even when exposed to high salinity levels. 


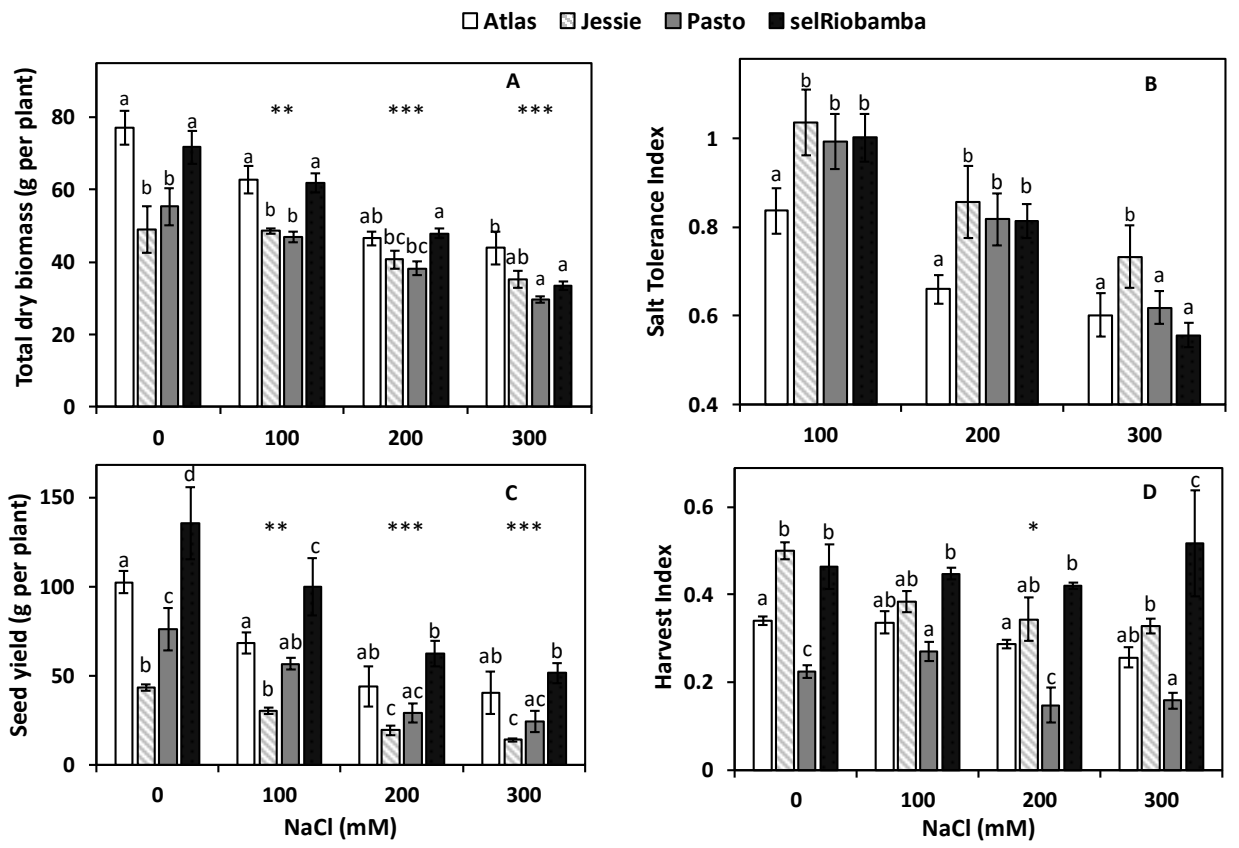

Figure 1. Agronomic characteristics of quinoa plants grown in Experiment 1 at various salinity levels. A) Total dry biomass weight 11 weeks after sowing, 6 weeks after salt application at the start of flowering. B) Salt tolerance index calculated as above biomass DW treatment/ above biomass DW control. C) Seed yield. D) Harvest index. Means of 5 plants. Error bars indicate SE of individual means. Statistically significant differences ( $p \leq 0.05)$ between varieties (within each salt treatment) are shown with different letters. Asterisks denote statistically significant differences $\left({ }^{*} p \leq 0.05,{ }^{* \star} p \leq 0.01,{ }^{* \star *} p \leq 0.001\right)$ between the salt treatments and the control.

\subsubsection{Ion contents}

Ion contents in the leaves were measured at the onset of flowering of the plants (6 weeks of salt treatment). Shoot $\mathrm{Na}^{+}$and $\mathrm{Cl}^{-}$concentrations increased significantly in plants under all salt treatments, with $\mathrm{Cl}^{-}$increasing much more than $\mathrm{Na}^{+}$(Figure 2A-B). Jessie had the highest accumulation of $\mathrm{Na}^{+}$and $\mathrm{Cl}^{-}$at all salt levels. Remarkably, the highest levels of shoot $\mathrm{Na}^{+}$and $\mathrm{Cl}^{-}$were not detected under the most severe $300 \mathrm{mM} \mathrm{NaCl}$ salt treatment. At $200 \mathrm{mM} \mathrm{NaCl}$, shoot $\mathrm{Na}^{+}$reached the maximum concentration of $213 \mathrm{mM}$ and $\mathrm{Cl}^{-}$of $553 \mathrm{mM}$. Pasto displayed the lowest $\mathrm{Na}^{+}$and $\mathrm{Cl}^{-}$concentrations in all the treatments. $\mathrm{K}^{+}$concentrations in the leaves were increased in all the salt treatments. Jessie had the highest shoot $\left[\mathrm{K}^{+}\right]$ under salinity, followed by Pasto (Figure $2 \mathrm{C}$ ). The latter had also the lowest levels of $\mathrm{Na}^{+}$and $\mathrm{Cl}^{-}$; as a result, it had the highest $\mathrm{K}^{+} / \mathrm{Na}^{+}$ratio in all the treatments, with the lowest value (6) at the most severe salt stress (Figure 2D). 


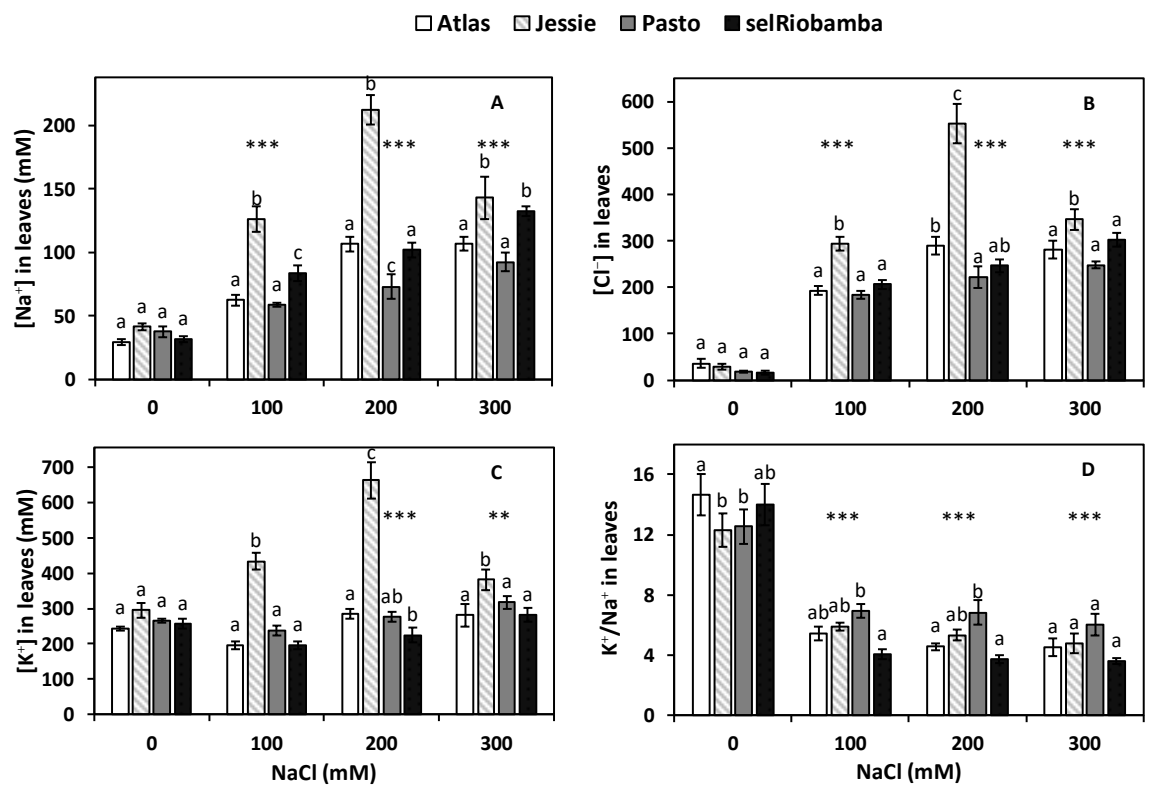

Figure 2. Ion contents in young leaves of plants grown in Experiment 1, 11 weeks after sowing, 6 weeks after salt application. A) $\left[\mathrm{Na}^{+}\right]$. B) $\left[\mathrm{Cl}^{-}\right]$. C) $\left[\mathrm{K}^{+}\right]$. D) $\mathrm{K}^{+} / \mathrm{Na}^{+}$. Means of 5 plants. Error bars indicate $\mathrm{SE}$ of individual means. Statistically significant differences $(p \leq 0.05)$ between varieties (within each salt treatment) are shown with different letters. Asterisks denote statistically significant differences ${ }^{*} p \leq 0.05$, ${ }^{* *} p \leq 0.01,{ }^{* * *} p \leq 0.001$ ) between the salt treatments and the control.

\subsection{Experiment 2: Full plant cycle response of quinoa cultivars to extreme salinity}

We further evaluated the impact of extreme salinity on quinoa in Experiment 2. Plants were treated with irrigation solutions containing 300 or $400 \mathrm{mM} \mathrm{NaCl}$. The soil substrate (vermiculite $\mathrm{nr} 1$ ) used in this experiment had a very high water-holding capacity and minimal drainage, and in order to prevent anoxia of the roots, the frequency of irrigations for the treated plants had to be lowered to once every ten days, leading to gradual accumulation of salt in the pots. At the end of the season, the EC of the $300 \mathrm{mM} \mathrm{NaCl}$-treated pots reached $\sim 55 \mathrm{dS} / \mathrm{m}$, and the $400 \mathrm{mM}$ treatment reached $65 \mathrm{dS} / \mathrm{m}$. The salinity level applied in this experiment was therefore substantially higher than in the first experiment, and exceeded the maximum salinity level at which quinoa was reported to still produce grain (Razzaghi et al. 2015). To facilitate comparison between experiments and treatment levels, the $300 \mathrm{mM} \mathrm{NaCl}$ irrigation treatment will be further referred to as $55 \mathrm{dS} / \mathrm{m}$ treatment and the $400 \mathrm{mM} \mathrm{NaCl}$ as $65 \mathrm{dS} / \mathrm{m}$ treatment. 
At the first destructive harvest 6 weeks after salt application (onset of flowering), Atlas had the highest biomass under control conditions ( $63 \mathrm{~g} / \mathrm{plant}$ ), followed by Red Carina, selRiobamba, Jessie and Pasto (Figure 3A). At this time, the salt treatments already had a considerable effect on the total dry biomass of all the varieties, but the difference in biomass between the two salt treatments was small. The total biomass mean was $17 \mathrm{~g}$ per plant for the $55 \mathrm{dS} / \mathrm{m}$ treatment and $15 \mathrm{~g}$ per plant for the 65 $\mathrm{dS} / \mathrm{m}$ treated plants and this resulted in a mean biomass-based salt tolerance index of 0.34 at $55 \mathrm{dS} / \mathrm{m} \mathrm{NaCl}$ irrigation and 0.27 at $65 \mathrm{dS} / \mathrm{m}$ (Figure 3B). As depicted in Figure $3 \mathrm{C}$, seed yield of the cultivars was reduced by $95 \%$ at $55 \mathrm{dS} / \mathrm{m}$ and by $97 \%$ at $65 \mathrm{dS} / \mathrm{m} \mathrm{NaCl}$. Despite the strong reduction in biomass, all the plants survived and produced seed.

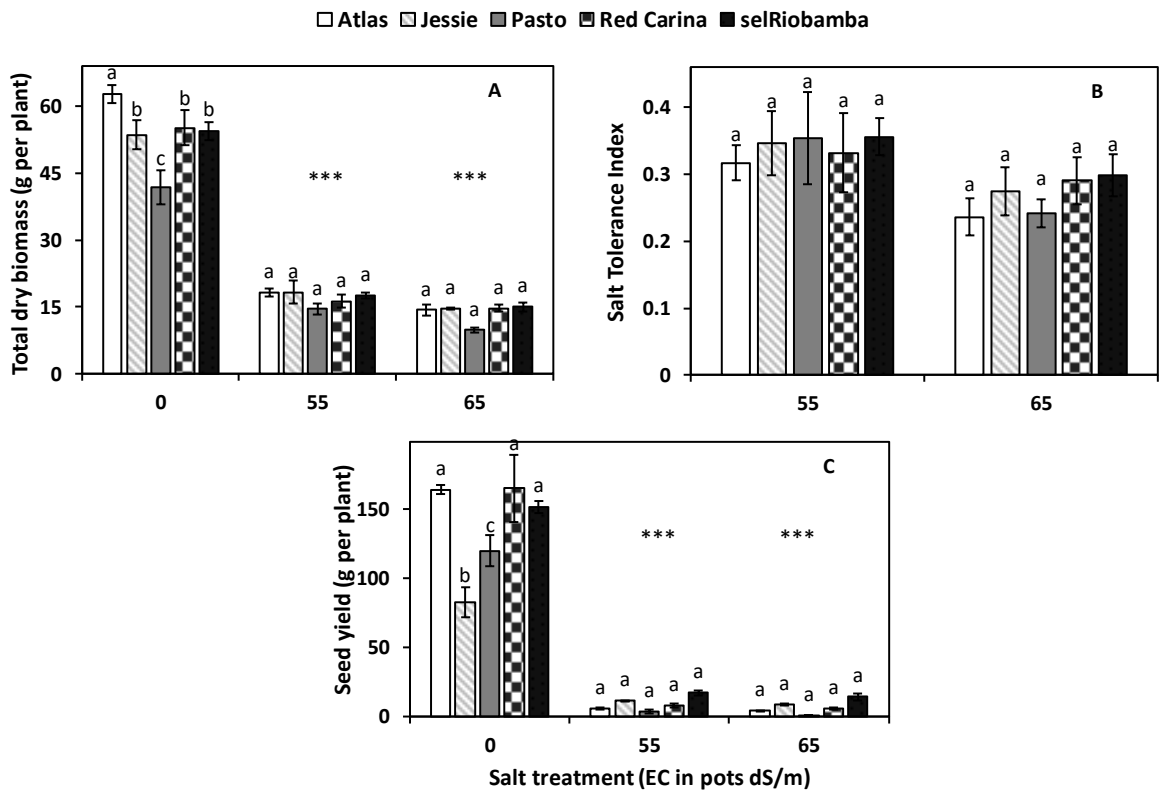

Figure 3. Agronomic characteristics of quinoa plants grown in Experiment 2 at high concentrations of salt. A) Total dry biomass weight 11 weeks after sowing, 6 weeks after salt application. B) Salt tolerance index calculated as above biomass DW treatment/ above biomass DW control. C) Seed yield. Means of 4 plants. Error bars indicate SE of individual means. Statistically significant differences $(p \leq 0.05)$ between varieties (within each salt treatment) are shown with different letters. Asterisks denote statistically significant differences $\left({ }^{*} p \leq 0.05,{ }^{* *} p \leq 0.01,{ }^{* * *} p \leq 0.001\right)$ between the salt treatments and the control. 
Ion concentrations in young leaves were quite different between the two salt treatments (Figure 4). Curiously, $\mathrm{Na}^{+}$and $\mathrm{Cl}^{-}$concentrations were higher under 55 $\mathrm{dS} / \mathrm{m}$ compared to the $65 \mathrm{dS} / \mathrm{m}$ treatment. Mean $\left[\mathrm{Na}^{+}\right]$was $48 \%$ higher at $55 \mathrm{dS} / \mathrm{m}$ than at $65 \mathrm{dS} / \mathrm{m}$ and $\left[\mathrm{Cl}^{-}\right]$was $28 \%$ higher. Interestingly, [ $\left.\mathrm{K}^{+}\right]$was also increased (by $35 \%)$ under the severest salinity stress (EC of $65 \mathrm{dS} / \mathrm{m}$ ) compared to control.

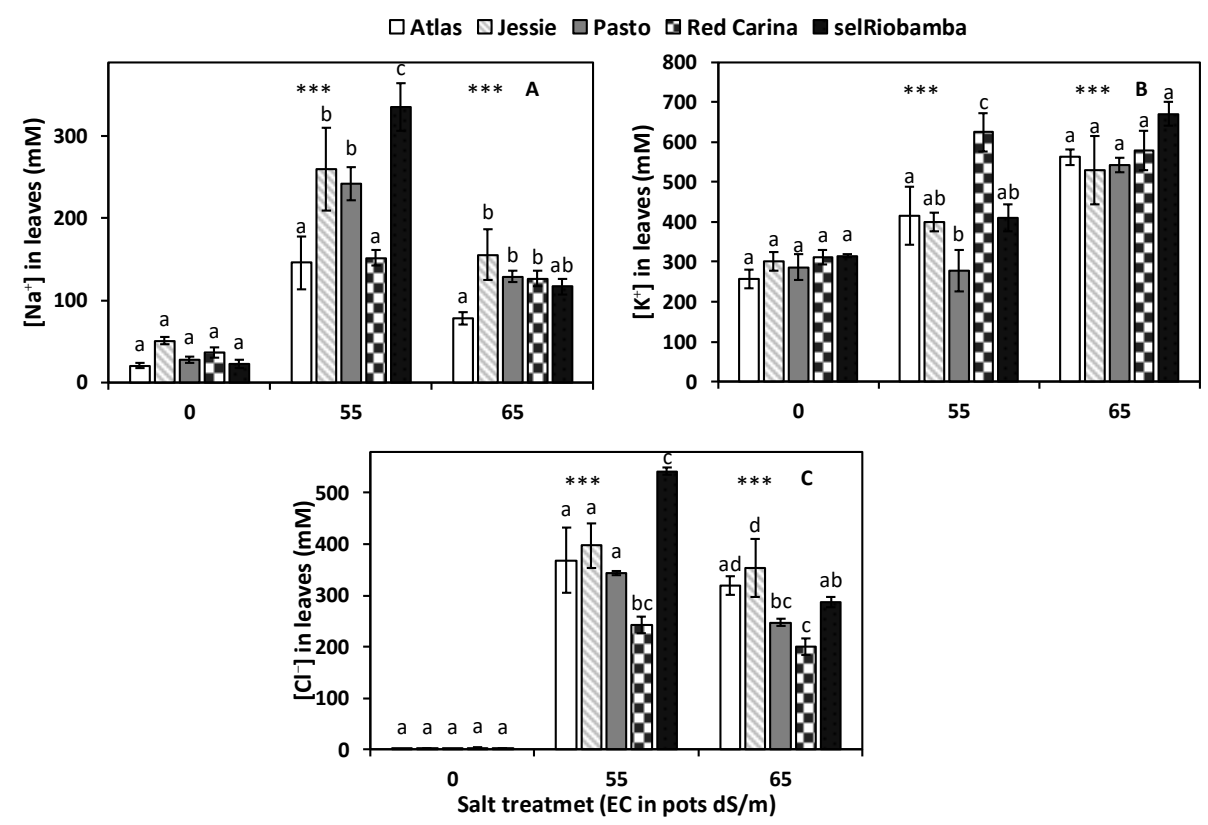

Figure 4. Ion contents in young leaves of plants grown in Experiment 2 at high concentrations of salt (11 weeks after sowing, 6 weeks after salt application). A) $\left[\mathrm{Na}^{+}\right]$. B) $\left[\mathrm{K}^{+}\right]$. C) $\mathrm{K}^{+} / \mathrm{Na}^{+}$. Means of 4 plants. Error bars indicate $S E$ of individual means. Statistically significant differences $(p \leq 0.05)$ between varieties (within each salt treatment) are shown with different letters. Asterisks denote statistically significant differences $\left({ }^{*} p \leq 0.05,{ }^{* *} p \leq 0.01,{ }^{* * *} p \leq 0.001\right)$ between the salt treatments and the control.

\subsection{Experiment 3: Detailed evaluation of the growth, ion dynamics and physiology of quinoa exposed to high salinity $(400 \mathrm{mM} \mathrm{NaCl})$}

\subsubsection{Biomass under high salinity}

In Experiment 3, plant height under control and highly saline conditions (400 $\mathrm{mM} \mathrm{NaCl}$ ) was measured weekly during the whole growing season (Figure $5 \mathrm{~A}$ ). The height of plants started to be affected two weeks after salt application and this difference became significant one week later. From this time point onwards, the 
control plants increased their height until eight weeks after the start of the salt treatment, while the height of the salt-treated plants increased at a lower rate and stopped increasing earlier. At the end of the season, this resulted in a $40 \%$ lower plant height at $400 \mathrm{mM} \mathrm{NaCl}$ compared to $0 \mathrm{mM}$ (average for the three cultivars). Destructive harvests throughout the whole crop cycle allowed monitoring the effect of salinity on the biomass of plants at different stages of development. Four weeks after the start of the salt treatment, the STI (for shoot dry biomass) at $400 \mathrm{mM}$ salt was only $50 \%$ and the effect of salt increased strongly with time. After ten weeks of salt treatment Jessie had the highest STI (31\%), followed by selRiobamba (26\%) and Pasto (20\%). This differential response was stronger for the STI based on seed yield at the end of the season. Jessie had the highest seed-based STI of $19 \%$, followed by selRiobamba with $13 \%$ and Pasto had the lowest of only $2 \%$ : Pasto survived well (small but green plants), but hardly produced grain (Figure 5B). Yield parameters including thousand seed weight (TSW) and harvest index $(\mathrm{HI})$ are shown in Figure 6A-C. Similar to Experiment 2, seed yield was strongly compromised at $400 \mathrm{mM} \mathrm{NaCl}$ salinity (average reduction of $88 \%$ ). TSW was reduced for all varieties (average mean reduction $34 \%$ ) with Pasto having the lowest values. Remarkably, the harvest index of selRiobamba and Jessie was not significantly influenced by the salt treatment. Pasto's harvest index however was strongly reduced by $83 \%$. 

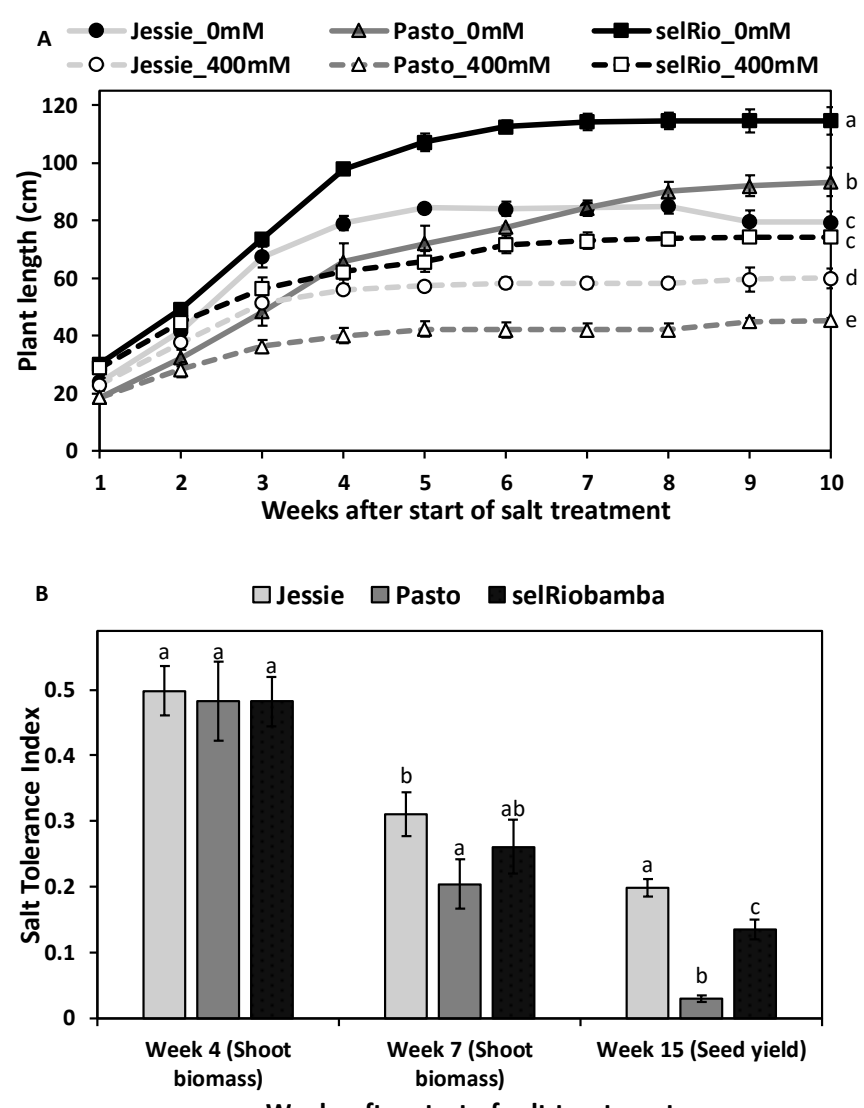

Figure 5. Growth responses of quinoa growing at $400 \mathrm{mM} \mathrm{NaCl}$ in Experiment 3. A) Plant height development through the season. Means of three plants. Error bars indicate SE of individual means. Statistically significant differences $(p \leq 0.05)$ between any variety and salt treatment combination are shown with different letters. B) Salt tolerance index in different harvests through the season based on biomass of plants. Means of 3 plants. Error bars indicate SE of individual means. Statistically significant differences $(p \leq 0.05)$ between varieties for each time point are shown with different letters. 


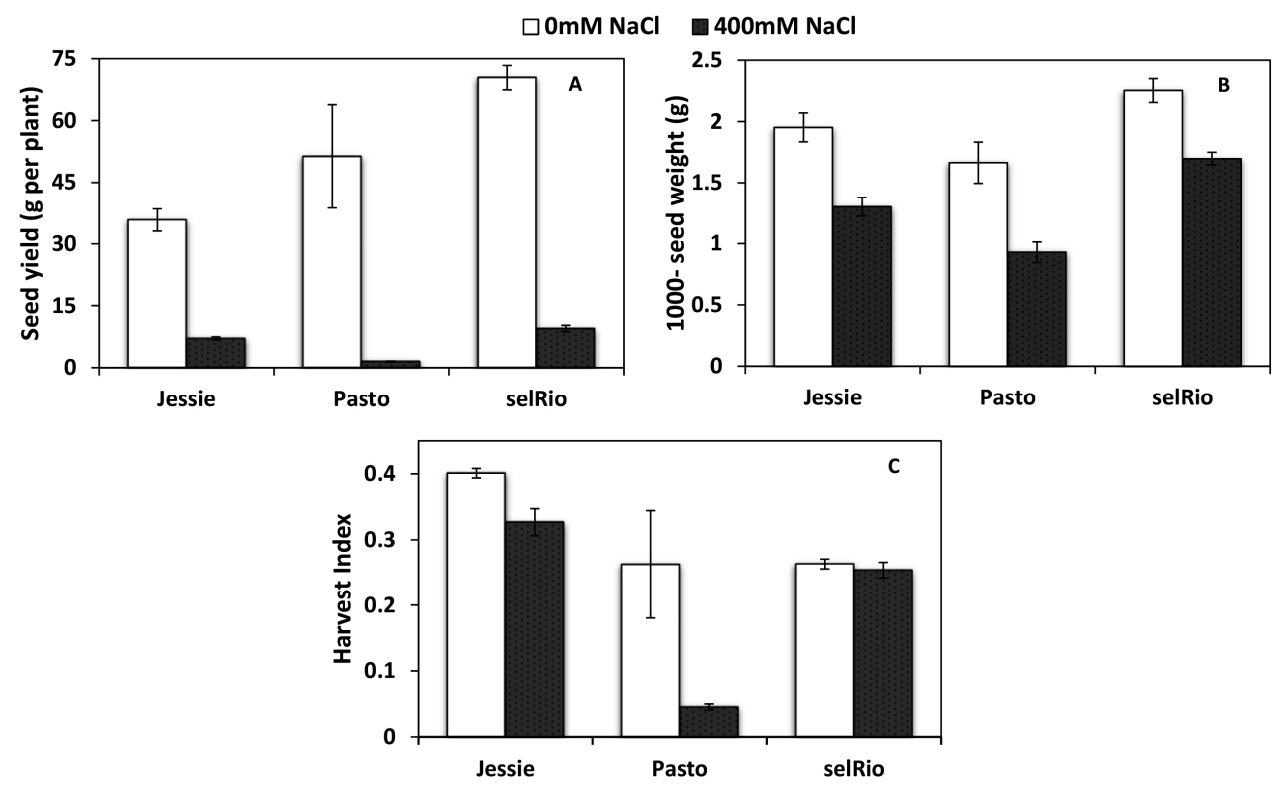

Figure 6. Yield traits of quinoa growing at $400 \mathrm{mM} \mathrm{NaCl}$ in Experiment 3. A) Seed yield. B) Thousand seed weight. C) Harvest index. Means of 3 plants. Error bars indicate SE of individual means. Statistically significant differences $(p \leq 0.05)$ between any variety and salt treatment combination are shown with different letters.

\subsubsection{Effect of high salinity on ion dynamics}

3.3.2.1 Roots. After 4 weeks of salt stress, the concentrations of $\mathrm{Na}^{+}$and $\mathrm{Cl}^{-}$in the roots were increased in all varieties (mean $\left[\mathrm{Na}^{+}\right]: 62 \mathrm{mM}$ and [Cl-]: $42 \mathrm{mM}$, compared to $4 \mathrm{mM}$ and below the detection level, respectively, under control conditions), but still much lower than the $400 \mathrm{mM} \mathrm{NaCl}$ concentration in the root medium. While no significant differences were observed between cultivars at the first time point, after seven weeks of the salt treatment the root $\left[\mathrm{Na}^{+}\right]$was higher than that of the root medium for Jessie and selRiobamba ( $452 \mathrm{mM}$ and $517 \mathrm{mM}$, respectively) but not for Pasto $(370 \mathrm{mM})$. Root $\left[\mathrm{Cl}^{-}\right]$was lower than that of $\mathrm{Na}^{+}$and remained lower than in the root medium (mean $=243 \mathrm{mM}$ ). Pasto had the lowest root accumulation of $\mathrm{Na}^{+}$ and $\mathrm{Cl}^{-}$in the first seven weeks of treatment, but these were higher and close to those of the other cultivars at the last measured time point, 10 weeks after the start of salt application (415 and $253 \mathrm{mM}$, respectively) (Figure 7A-B).

The $\left[\mathrm{K}^{+}\right]$in roots of plants grown at $400 \mathrm{mM} \mathrm{NaCl}$ was relatively stable throughout the season. Among the varieties, Pasto always had the highest $\left[\mathrm{K}^{+}\right]$. Pasto was also the only variety with higher $\left[\mathrm{K}^{+}\right]$under salt stress compared to the control after four weeks of salt treatment. For all varieties, the $\mathrm{K}^{+} / \mathrm{Na}^{+}$ratio in the salt-treated roots was already reduced at four weeks after the start of the salt 
treatment and remained lower than 1.0 throughout the season. In comparison, in roots of control plants this index was always above 4 (Figure 7C-D).

3.3.2.2. Leaves. Ion concentrations were measured in young and old leaves separately. No significant differences were found between both (Figure S1). The ion contents of the young leaves are presented in the following section, but these are representative of total leaves ion contents.

$\mathrm{Na}^{+}$and $\mathrm{Cl}^{-}$accumulated in the leaves of salt-stressed plants over time, but in contrast to the roots, $\left[\mathrm{Cl}^{-}\right]$was higher than $\left[\mathrm{Na}^{+}\right]$in all varieties at all-time points (Figure 7E-F). For the first two time points, Pasto had the lowest $\mathrm{Na}^{+}$and $\mathrm{Cl}^{-}$ concentrations in the leaves. After ten weeks of treatment, the concentration of both ions reached similar and very high values in all three cultivars; the average [ $\left.\mathrm{Na}^{+}\right]$was $667 \mathrm{mM}$ and the average $\left[\mathrm{Cl}^{-}\right]$was $755 \mathrm{mM}$. Remarkably, the salt treatment also caused an increase in $\left[\mathrm{K}^{+}\right]$in leaves in all the time points and varieties (Figure $7 \mathrm{G}$ ). Consequently, the $\mathrm{K}^{+} / \mathrm{Na}^{+}$decreased but remained relatively high throughout the growing season (Figure $7 \mathrm{H}$ ), with an average of 1.5 for the three varieties after 10 weeks of stress. Pasto had the highest shoot $\left[\mathrm{K}^{+}\right]$and lowest $\left[\mathrm{Na}^{+}\right]$and therefore the highest $\mathrm{K}^{+} / \mathrm{Na}^{+}$in the shoot. 

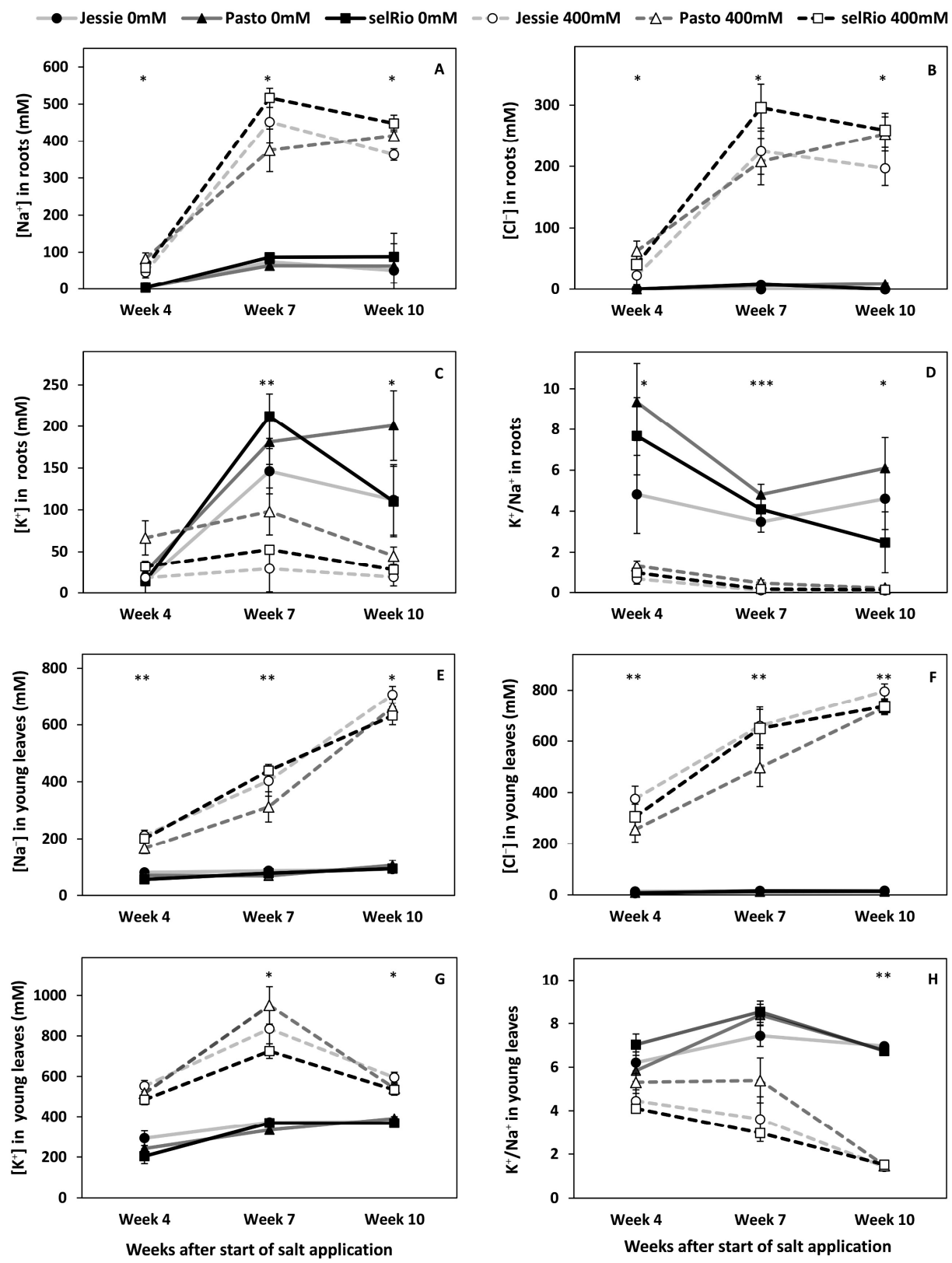

Figure 7. $\mathrm{Na}^{+}, \mathrm{K}^{+}, \mathrm{Cl}^{-}$contents and $\mathrm{K}^{+} / \mathrm{Na}^{+}$ratio in roots and young leaves measured throughout the growing season in plants grown in Experiment 3. Means of 3 plants. Error bars indicate SE of individual means. Asterisks denote statistically significant differences $\left({ }^{*} p \leq 0.05,{ }^{* *} p \leq 0.01,{ }^{* * *} p \leq 0.001\right)$ between the salt treatments and the control. 
Figure $8 \mathrm{~A}-\mathrm{C}$ depicts the distribution of a number of inorganic anions and cations $\left(\mathrm{Na}^{+}, \mathrm{K}^{+}, \mathrm{Ca}^{2+}, \mathrm{Mg}^{2+}, \mathrm{Cl}^{-}, \mathrm{PO}_{4}{ }^{3-}, \mathrm{SO}_{4}{ }^{2-}, \mathrm{NO}_{3}{ }^{-}\right)$over roots, stems and young leaves seven weeks after the start of salt treatment. The concentration of $\mathrm{Cl}^{-}$in salttreated plants showed an increasing gradient from root to stem to leaves (mean [ $\left.\mathrm{Cl}^{-}\right]$: $243 \mathrm{mM}$ in roots, $396 \mathrm{mM}$ in stems and $580 \mathrm{mM}$ in leaves). $\mathrm{Na}^{+}$accumulation in leaves was much lower than $\mathrm{Cl}^{-}$accumulation, and lower than $\mathrm{Na}^{+}$accumulation in the other two tissues (448 $\mathrm{mM}$ in roots, $525 \mathrm{mM}$ in stems and $369 \mathrm{mM}$ in leaves), suggesting an active exclusion of $\mathrm{Na}^{+}$from leaves. Root $\left[\mathrm{K}^{+}\right]$was reduced by the $\mathrm{NaCl}$ treatment, while in the stem $\left[\mathrm{K}^{+}\right]$was $25 \%$ higher in treated plants compared to the controls, and in the leaves it was increased by $133 \%$. Similar to $\mathrm{K}^{+}, \mathrm{Mg}^{2+}$ and phosphate concentrations were reduced in the roots, but not in the leaves. The concentration of $\mathrm{Ca}^{2+}$ was reduced by salinity in all the tissues. Sulphate was the least affected ion by the salt treatment. The concentration of nitrate was measured only in young leaves (Figure S2). Salt treatment caused a reduction of the nitrate content that substantially differed between varieties. Leaf nitrate in Pasto was hardly affected (7\% reduction), while selRiobamba had the highest reduction of $73 \%$. Interestingly, the electrical balance of inorganic ions was positive to a similar degree for both salt concentrations. This might imply that no additional energy is required for the synthesis of negative organic compounds under salt stress to restore the electrical neutrality. 


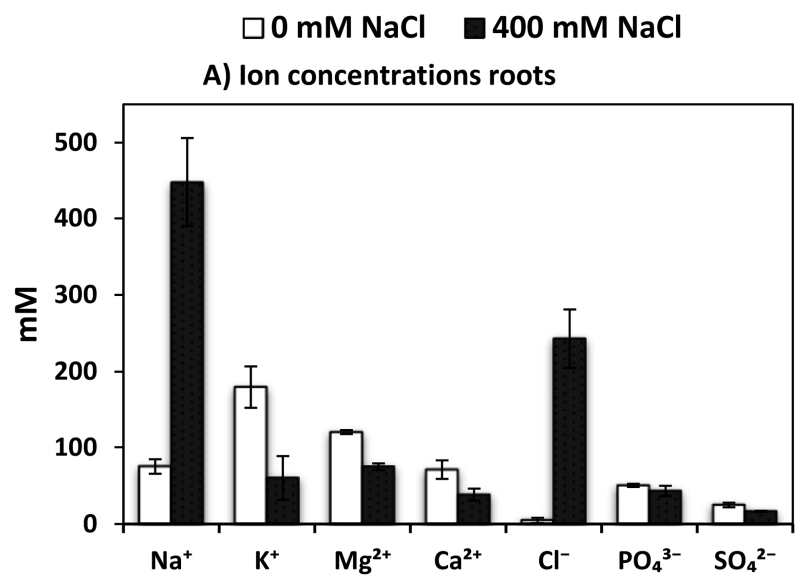

B) Ion concentrations stems
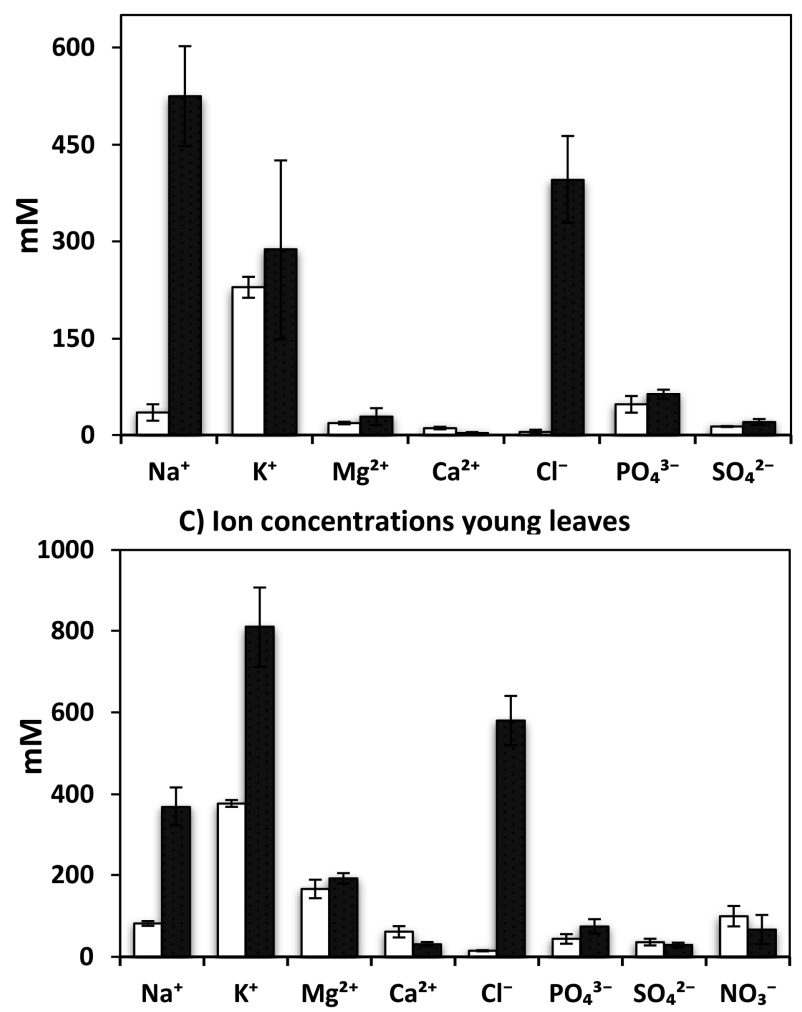

Figure 8. Ion contents in different tissues of quinoa grown in Experiment 3 at $400 \mathrm{mM} \mathrm{NaCl} 12$ weeks after sowing, 7 weeks after start of the salt treatment. A) Roots. B) Stems. C) Young leaves. Means of three varieties (Jessie, Pasto, selRiobamba) and 3 replicates per variety. Error bars indicate SE of individual means. Asterisks denote statistically significant differences $\left({ }^{*} p \leq 0.05,{ }^{* *} p \leq 0.01,{ }^{* * *} p \leq 0.001\right)$ between the salt treatment and the control for each ion content. 


\subsubsection{High salinity stress and plant physiology}

All the salt-treated plants showed significantly lower leaf stomatal conductance (gs) compared to control plants. However, the effect of salt on gs was only detected after two weeks of salt application ( $25 \%$ reduction of $g s$ ). Three weeks after the beginning of the treatment the effect of salinity on stomatal conductance became considerably more pronounced, with an average reduction of $60 \%$. Stomatal conductance values remained low during the rest of the growing period, with hardly any fluctuations due to weather conditions or physiological maturation. The highest $g s$ reduction was found in Pasto, followed by selRiobamba and Jessie (Figure 9A). Stomatal conductance was also measured five times over a 24-hr timespan (Figure 9B), at the onset of flowering (seven weeks after salt application). Under control conditions, daily gs was characteristic of a C3 crop during a summer day (middle of June) in the Northern Hemisphere. Sunrise occurred around 5:00 AM and this coincided with an increase in gs after very low levels during the night. During the day, gs increased reaching its maximum value around 3:00 PM, after which it declined and totally stopped after sunset (9:00 PM). The gs peak was also observed around 3:00 PM for stressed plants, but the conductance declined faster and was below the detection threshold of the porometer many hours before sunset. Hence, not only was the maximal gs decreased under salinity stress, but the time that stomata were opened during the day was also shortened. Maximum quantum yield of PSII (Fv/Fm) of quinoa leaves was not affected by severe salt stress (average value for control and salt treated plants was 0.79 ) (Figure S3). Chlorophyll content was measured throughout the growing season and was significantly influenced by salinity as well as genotype (Figure 9C). At the beginning of the treatment (one week after salt application) the chlorophyll content was higher in the salt treated plants. It remained higher throughout the whole season for Pasto, while for Jessie and selRiobamba it was reduced by salt after five weeks of salt treatment. Leaf RWC was significantly reduced ( $25 \%$ ) by salinity (Figure 9D). 

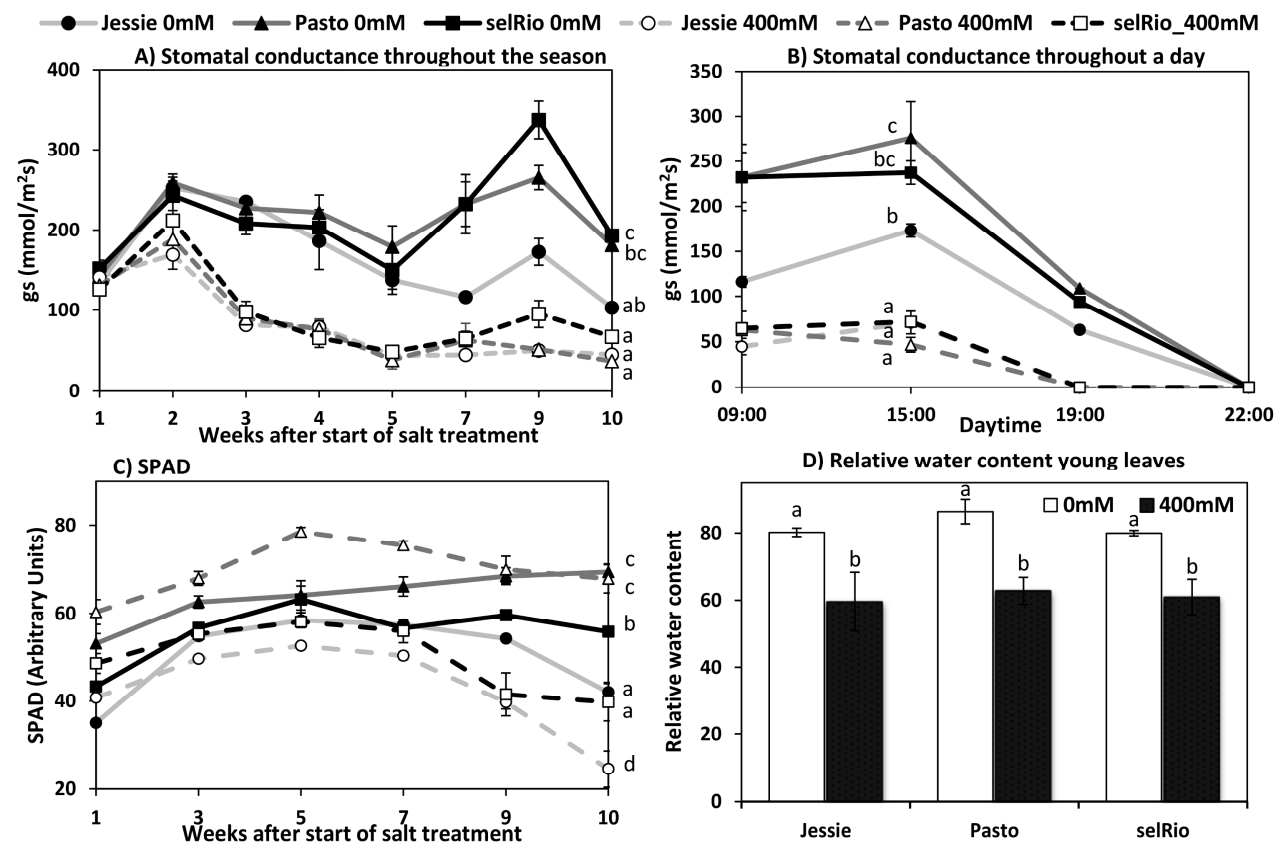

Figure 9. Physiological traits measured in quinoa grown at $400 \mathrm{mM} \mathrm{NaCl}$ in Experiment 3. A) Stomatal conductance through the growing season measured from 9:00 AM until 11:00 AM. B) Circadian variation of stomatal conductance. C) Chlorophyll content (SPAD measurements) through the growing season. D) Relative water content. Measurements depicted on $B$ and $D$ were taken 7 weeks after the start of salt treatment. Means of 3 plants. Error bars indicate SE of individual means. Statistically significant differences $(p \leq 0.05)$ between any variety and salt treatment combination are shown with different letters.

\subsubsection{High salinity stress and growth}

The mean RGR for all the cultivars was $0.111 \mathrm{~d}^{-1}$ at $0 \mathrm{mM} \mathrm{NaCl}$ and 0.0985 $\mathrm{d}^{-1}$ at $400 \mathrm{mM} \mathrm{NaCl}$ (Figure 10A). The difference in the leaf weight ratio (LWR) between treatments or cultivars was not significant (Fig. 10B). The most significant effect of the salt treatment on RGR components was a decrease in the specific leaf area (SLA), from 307 to $206 \mathrm{~m}^{2} \mathrm{~kg}^{-1}$ for 0 and $400 \mathrm{mM} \mathrm{NaCl}$ treatment, respectively (Figure 10C). The physiological component of RGR, net assimilation rate (NAR), was surprisingly increased under saline conditions, from an average $13 \mathrm{~g} \mathrm{~m}^{-2} \mathrm{~d}^{-1}$ in control plants to $16 \mathrm{~g} \mathrm{~m}^{-2} \mathrm{~d}^{-1}$ in salt treated plants (Figure 10D). 

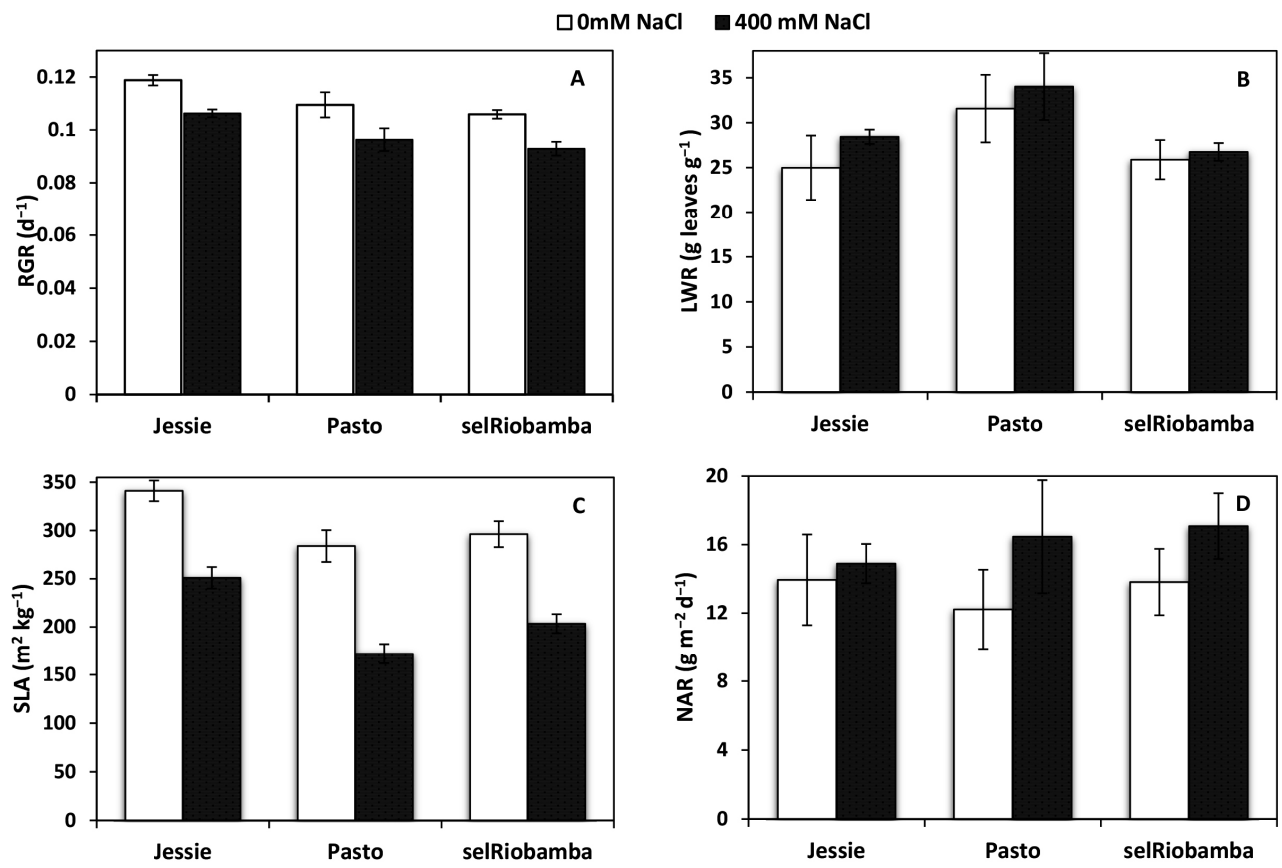

Figure 10. Effect of severe salt stress $(400 \mathrm{mM} \mathrm{NaCl})$ on Relative Growth Rate components of plants grown in Experiment 3 from the transplanting date ( 3 weeks after sowing) until 4 weeks after beginning of salt treatment (9 weeks after sowing). A) Relative growth rate (RGR). B) Leaf weight ratio (LWR). C) Specific leaf area (SLA). D) Net assimilation rate (NAR). Means of 3 plants. Error bars indicate SE of individual means. Statistically significant differences $(p \leq 0.05)$ between any variety and salt treatment combination are shown with different letters.

\subsection{Contribution of epidermal bladder cells to salt tolerance}

Salt stress reduced the water content of epidermal bladder cells (Figure 11A). We measured the content of $\mathrm{Na}^{+}, \mathrm{Cl}^{-}$and $\mathrm{K}^{+}$in 1) young leaves including EBCs, 2) young leaves after the removal of EBCs, and 3) brushed EBCs (Figure 11B). The results were expressed as the amount of ions $(\mathrm{mmol})$ per area of leaves $\left(\mathrm{m}^{2}\right)$, or in the EBCs removed from the same leaf area. This allowed the assessment of the relative contribution of EBCs to ion storage compared to the total amount of ions accumulated in the leaves. The concentration of $\mathrm{Na}^{+}, \mathrm{Cl}^{-}$and $\mathrm{K}^{+}$in the bladders was higher in the salt-treated plants compared to the controls (Figure 11B). However, the percentage of ions accumulated in the EBCs relative to the total leaf ion content was only $5.4 \%$ for $\mathrm{Na}^{+}, 6.5 \%$ for $\mathrm{Cl}^{-}$and $15 \%$ for $\mathrm{K}^{+}$. The relatively high accumulation of $\mathrm{K}^{+}$in the bladders coincides with the high levels of this ion distributed in all the leaf tissue. Based on the results in our study, storage of salt in EBCs is not likely to contribute significantly to reduce levels of $\mathrm{Na}^{+}$and $\mathrm{Cl}^{-}$in the leaves. 
A) Leaf biomass composition

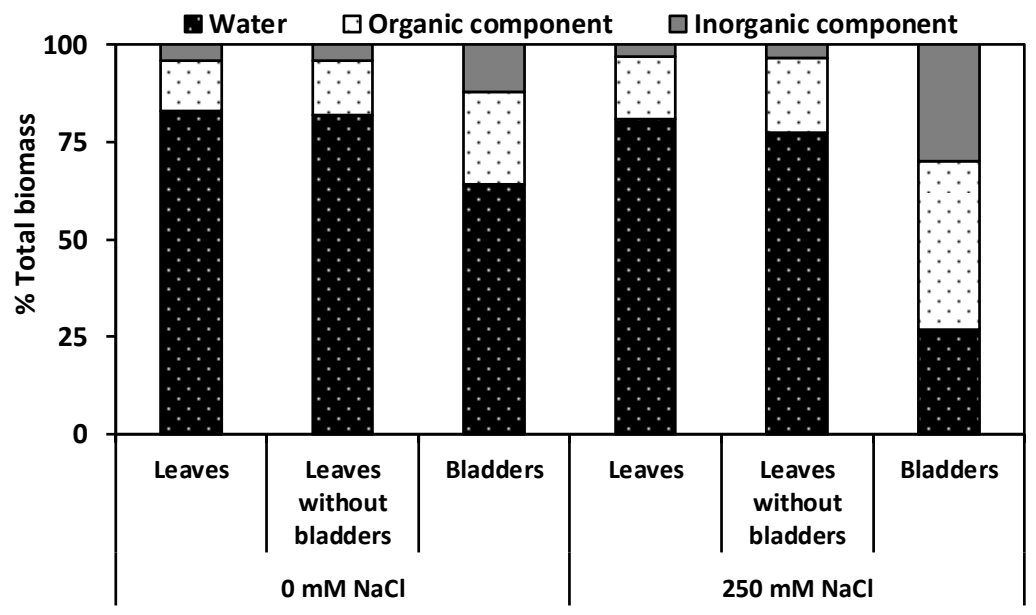

B) Ion content in leaves and bladders

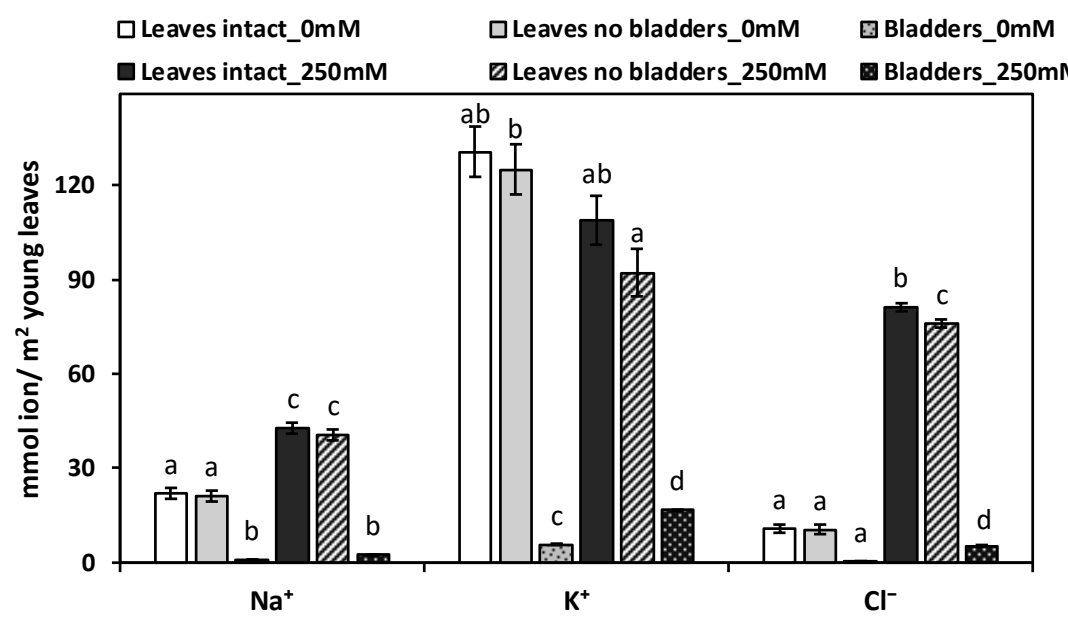

Figure 11. Epidermal bladder cells (EBCs) composition in quinoa plants (variety Pasto) grown at $250 \mathrm{mM}$ $\mathrm{NaCl}$. A) Leaf and bladders biomass composition under control and stress conditions. B) $\mathrm{Na}^{+}, \mathrm{K}^{+}$and $\mathrm{Cl}^{-}$ contents in leaves and bladders presented as the content of ions $(\mathrm{mmol})$ per $\mathrm{m}^{2}$ leaf area. Means of 5 biological replicates. Error bars indicate SE of individual means. Statistically significant differences $(p \leq 0.05)$ between any tissue and salt treatment combination (for each specific ion) are shown with different letters. 


\section{Discussion}

To fully understand why quinoa can survive and reproduce in highly saline conditions while being an economically productive food crop under normal, nonsaline conditions, it is essential to gain insight in the physiological changes and adaptations during the crop cycle under prolonged exposure to high salt levels in the soil. Our study demonstrates that quinoa varieties utilize salt exclusion strategies to produce relatively high yields under mild salinity or short-term stress, while tissue tolerance mechanisms enable the plants to survive and even reproduce under severe and prolonged salinity.

\subsection{Ion and water dynamics throughout the growing season}

Given the importance of water availability for all aspects of plant physiology, plants suffer from salinity first and foremost because of the problems with water uptake. Water uptake from the root medium is a complex process mediated by longdistance shoot-to-root signals. Under saline conditions, water uptake and transport in the plant is also influenced by $\mathrm{Na}^{+}$and $\mathrm{Cl}^{-}$uptake and distribution over the plant tissues. In a two-step model, $\mathrm{Na}^{+}$passively enters root cells via non-selective cation channels driven by a negative membrane potential and a low $\left[\mathrm{Na}^{+}\right]$in the cytosol (Britto and Kronzucker 2015). According to this model, sodium ions rapidly exit the cells through the SOS1 transporter, the only cytoplasmic $\mathrm{Na}^{+}$efflux transporter identified until now. By monitoring the ion contents in different tissues throughout the growth of the plants we were able to identify specific, time- and stress leveldependent strategies used by quinoa to cope with salt stress. The complex ions dynamics described for quinoa is likely to be associated to the activity of key, possibly novel, ion transporters. The expression of only a few transporters (SOS1, $\mathrm{NHX}$ ) has been examined in short-term experiments in quinoa and variable responses between varieties have been reported (Maughan et al. 2009; RuizCarrasco et al. 2011; Schmöckel et al. 2017). An in silico exploration of ion transporters annotated in the genome of quinoa (Jarvis et al. 2017) revealed a high diversity and abundance of $\mathrm{K}^{+}, \mathrm{Na}^{+}$and $\mathrm{Cl}^{-}$transporters in quinoa compared to other species reported in literature (Lebaudy et al. 2007; Véry et al. 2014). A comprehensive study of the expression of these transporter families in different tissues at different developmental stages of the crop would help to elucidate the role of the ion transporters involved in the different salt tolerant strategies reported here, but such study is beyond the scope of this manuscript.

In an early stage of development (budding) and relatively short exposure to high salt stress (4 weeks of $400 \mathrm{mM} \mathrm{NaCl}$ ) the concentration of $\mathrm{Na}^{+}$and $\mathrm{Cl}^{-}$in the roots 
was considerably lower than in the soil medium (Figure 12, upper panel), suggesting that the plants actively excluded the ions from the roots. This may represent a substantial challenge to the plants: the water needs to be taken up by the roots against an ion gradient. Stomatal conductance in our study was not significantly reduced until three weeks after the stress was imposed, indicating that the quinoa varieties were not saving water but maintained high photosynthesis and growth rates during the first two weeks of exposure to high salinity. This implies that during this first stage of salt stress, quinoa plants were still able to take up water from the root medium, which suggests that osmotic adjustment most likely relied on the production of organic osmolytes. The contribution of these organic osmolytes for osmotic adjustment in quinoa was previously reported (Shabala et al. 2012).

Our results indicate that at flowering (7 weeks after beginning of the stress), the plants have changed strategies (Figure 12, middle panel). At this time, $\left[\mathrm{Na}^{+}\right]$in the roots equalled that of the root medium $(400 \mathrm{mM})$, while the $\mathrm{Cl}^{-}$was still lower $(250 \mathrm{mM})$, and remained like that until the end of the season. The increased $\left[\mathrm{Na}^{+}\right]$in the root tissues helps to restore the osmotic balance with the root environment, facilitating water uptake. However, at this stage, stomatal conductance was considerably lower than early in the season. Previous reports showed that quinoa reduced transpiration under salt stress at similar stages of development and stress levels (Adolf et al. 2012; Orsini et al. 2011). Decreased transpiration will reduce the rate of $\mathrm{Na}^{+}$and $\mathrm{Cl}^{-}$accumulation the leaves. Munns et al. (2020) reported that most plant species are able to exclude about $98 \%$ of the salt in the root medium, but in spite of this the salt concentrations in the shoot will still be equal to that of the root medium after 3 to 4 weeks. In most of our varieties, the shoot $\mathrm{Na}^{+}$and $\mathrm{Cl}^{-}$ concentrations were lower than the root medium at 4 weeks, suggesting a high level of root $\mathrm{Na}^{+}$exclusion in quinoa, but at 7 weeks a similar concentration than the root media was reached. $\left[\mathrm{Na}^{+}\right]$in the shoot of the variety Pasto however was still lower. From this stage onwards, quinoa appears to mostly rely on tissue tolerance to cope with salinity. High levels of $\mathrm{Na}^{+}$and $\mathrm{Cl}^{-}$in the cytoplasm are detrimental for cells (Maathuis et al. 2014); therefore, vacuolar compartmentalization of these ions is a likely strategy in quinoa at prolonged and high levels of salinity. The sequestration of $\mathrm{Na}^{+}$and $\mathrm{Cl}^{-}$in the vacuole not only protects the cytoplasm against toxicity, but also increases the osmotic potential of the cell in a cost-effective manner, as long as the cytosolic osmotic potential is adjusted accordingly. Our results supports previous indications that $\mathrm{K}^{+}$may play an important role in this adjustment (Rubio et al. 2020). While in the roots $\mathrm{K}^{+}$concentration was decreased in stressed plants compared to controls from the earliest time point measured until harvest, it was higher in young 
leaves of salt-stressed plants than in control leaves and even higher than the $\left[\mathrm{Na}^{+}\right]$. In our experiments, quinoa appeared to be able to maintain leaf cell turgor (no signs of wilting) even when leaf RWC was reduced, which is indicative of a strategy of osmotic adjustment (Negrão et al. 2017).

Towards the end of the growing cycle (seed filling, 10 weeks after stress application) $\mathrm{Na}^{+}$and $\mathrm{Cl}^{-}$concentrations were further increased in the aboveground tissues, and a clear positive concentration gradient of ions was observed from roots to stems to leaves (Figure 12, lower panel). The active $\mathrm{Na}^{+}$exclusion early in the season changed into a strong building up of ions in the latter stages of development. It is worth to note that at this stage gs was strongly reduced, reaching values even below the detection level of the porometer. It is conceivable that at prolonged exposure to high salinity, the plants can no longer maintain low shoot $\mathrm{Na}^{+}$and $\mathrm{Cl}^{-}$ levels through ion exclusion from the roots, but accumulation in the leaves to extreme levels that are toxic even for quinoa are avoided by minimizing the transpiration stream that transports ions to the leaves. 


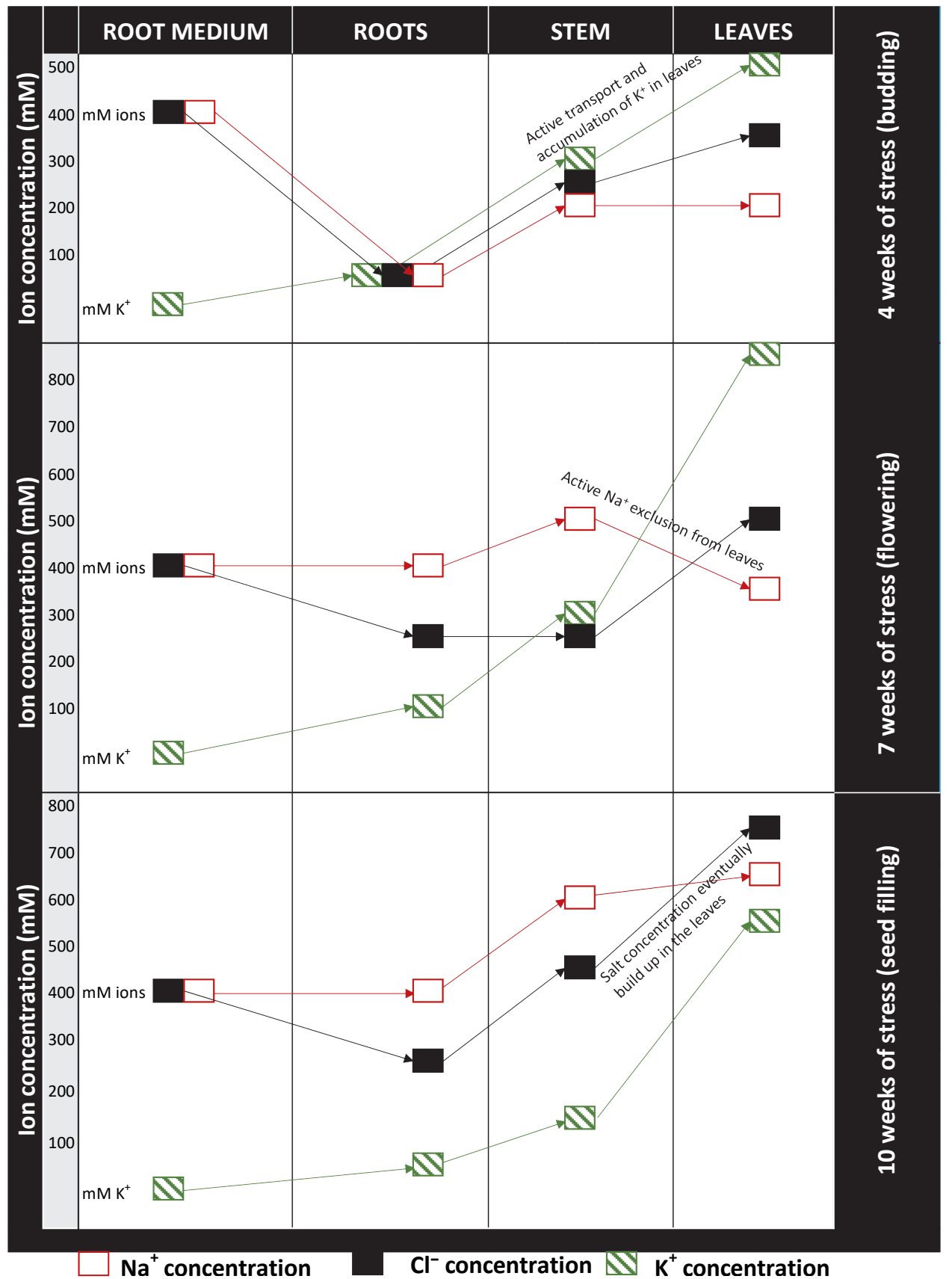

Figure 12. Schematic representation of ion dynamics in quinoa throughout the growth cycle based on ion content measurements in different tissues. 
It is noteworthy that $\left[\mathrm{Cl}^{-}\right]$in young leaves was higher that $\left[\mathrm{Na}^{+}\right]$in the shoots throughout the season. The high values for $\left[\mathrm{Cl}^{-}\right]$reported in this study agree with reports in other halophytes. $\mathrm{Cl}^{-}$accumulation has been considered as a compensatory mechanism to prevent charge imbalances (Flowers and Colmer 2008). Given the high concentration of monovalent cations $\left(\mathrm{Na}^{+}\right.$and $\left.\mathrm{K}^{+}\right), \mathrm{Cl}^{-}$might be essential for electrical balance and the maintenance of a negative voltage (cytoplasm with respect to apoplast) (Teakle and Tyerman 2010). Some of the consequences of $\mathrm{Cl}^{-}$accumulation can be inhibition of gas exchange and reduction of nitrogen uptake and nitrate storage due to competitive transport of $\mathrm{Cl}^{-}$and $\mathrm{NO}_{3}{ }^{-}$ (Li et al. 2017). In our study, salt stressed plants showed lowered gas exchange measured by stomatal conductance. However, free nitrate content in photosynthetically active leaves was strongly reduced only in selRiobamba (by 73\%) and mildly reduced in Pasto and Jessie (by $7 \%$ ), while $\mathrm{Cl}^{-}$concentration in the three varieties was similar. This suggests that the competition of $\mathrm{Cl}^{-}$and $\mathrm{NO}_{3}{ }^{-}$transport is not a general phenomenon in quinoa. Maintaining a high nitrate level in the leaves was not a determinant factor for the salt tolerance of these varieties. The lowest nitrate level under salt stress was found in selRiobamba, which showed the lowest seed yield reduction under severe salt stress.

When plants are transferred to a medium with high $\mathrm{Na}^{+}$(salt treatment), plant $\left[\mathrm{K}^{+}\right]$typically decreases as $\left[\mathrm{Na}^{+}\right]$rises (Flowers and Colmer 2008). A major growth constraint of salt stress is a $\mathrm{Na}^{+}$- induced $\mathrm{K}^{+}$deficiency that can disrupt cell metabolism. Our quinoa plants were able to maintain and even increase the levels of $\mathrm{K}^{+}$in the shoot also at the high $\left[\mathrm{Na}^{+}\right]$after prolonged salt stress (10 weeks after start of the stress). Maintained or even elevated $\mathrm{K}^{+}$concentrations under high salinity is consistent with previous reports (Hariadi et al. 2011; Schmöckel et al. 2017; Shabala et al. 2013) and has been interpreted as evidence for the important role of $\mathrm{K}^{+}$in leaf osmotic adjustment under saline conditions (Shabala and Cuin 2008). It may also protect the cells from metabolic failure due to a low $\mathrm{K}^{+} / \mathrm{Na}^{+}$ratio. Mechanisms and transporters involved in the translocation of $\mathrm{K}^{+}$from root to shoot and its posterior distribution and cellular partitioning have been described in a number of studies (Ahmad and Maathuis 2014; Benito et al. 2014; Szczerba et al. 2009). Under these stressful conditions, the ability of quinoa to retain a high concentration of $\mathrm{K}^{+}$in the cytosol is remarkable and may be essential for its salt tolerance, though it may come at a high metabolic cost.

Some authors have proposed that under salt stress epidermal bladder cells (EBCs) act as external storage organs for potentially toxic ions, and that therefore EBCs would play a pivotal role in the ion homeostasis of quinoa (Orsini et al. 2011; 
Zou et al. 2017). The low amount of ions accumulated in EBCs relative to the total amount in the leaves in our study do not suggest a strong contribution of EBCs to reducing the high levels of $\mathrm{Na}^{+}$and $\mathrm{Cl}^{-}$in the leaves. EBCs constitute less than 1.3 $\%$ of the total fresh weight of young fully developed leaves. We think that the total EBCs volume is simply too low to hold enough salt to be considered salt storage organs under saline conditions. Further research is needed to understand the function of these specialized cells in quinoa.

\subsection{Long term salt stress effects on growth: components of RGR and PSII}

After the first three weeks of stress $(400 \mathrm{mM} \mathrm{NaCl})$ until the end of the crops cycle, stomatal conductance was reduced by more than $60 \%$. A similar reduction of the maximum $\mathrm{CO}_{2}$-assimilation rate can be expected, which might lead to the photoinhibition of PSII and additional non-stomatal limitations to photosynthetic efficiency (Murata et al. 2007). In plants, salinity typically causes a rapid decline of PSII activity due to the inhibition of the repair of PSII caused by excessive ROS production (Murata et al. 2007). However, despite the severe stress applied in this study, the initial PSII light use efficiency of our quinoa plants (Fv/Fm ratio) was not decreased (Figure S3) which corroborates previous reports in quinoa (Shabala et al. 2013).

While PSII efficiency was not affected by salt, the relative growth was. The impact of salt stress on quinoa growth during the full crop cycle can be assessed using Relative Growth Rate analysis (RGR) because it factorises growth into physiological, morphological, anatomical and biochemical traits (Lambers and Poorter 1992). The most important effect of salinity on quinoa RGR components was not on the relative investment in leaf growth (LWR), but on the morphology of the leaves (SLA); decreased SLA likely implies thicker leaves. The modified leaves have a decreased total area for transpiration and radiation interception, but increases photosynthetic capacity per surface area (as seen by an increased NAR). Therefore, both the initial light use efficiency (PSII) and the long term photosynthetic rate (NAR) were not affected by salt. A higher NAR associated with a lower SLA has been reported in several species (Montes Osorio et al. 2014). Gas exchange measurements reported after 4 weeks of the start of a salt treatment of $250 \mathrm{mM} \mathrm{NaCl}$ showed a lower maximum net $\mathrm{CO}_{2}$-assimilation rate (Becker et al. 2017). In our long term assessment, we observed a higher NAR, which suggest a recovery of $\mathrm{CO}_{2}$ assimilation after prolonged salt stress, even at lower stomatal conductance. This anatomical adaptation of quinoa leaves under severe stress (lower SLA with higher NAR) might explain that the RGR was reduced only by $10 \%$, a minor decrease 
compared to the impact of adverse conditions on the RGR of other species discussed in literature (Norris 1982).

In conclusion, salt stress impacts the growth of quinoa directly, through a lowered metabolism (stomatal closure, less carbon assimilation, decrease in cell expansion) and indirectly, through several salt tolerance mechanisms examined in this study that come at a considerable metabolic cost (Tyerman et al. 2019).

\subsection{Lessons from different stress levels: trade-off between survival and growth}

The European varieties in our study displayed remarkable variation in growth and salinity responses. The salt treatments in the range of $100-200 \mathrm{mM} \mathrm{NaCl}$ might be considered mild stress for quinoa; even though seed yield was reduced, quinoa was still able to perform relatively well under these conditions compared to other grain crops. Under mild stress, varieties did not differ in their responses to salt. Under highly saline conditions ( $>400 \mathrm{mM} \mathrm{NaCl}$ ), seed yield was severely reduced in all the varieties, but Pasto was the most affected. This variety displayed a behaviour that deviated from the other varieties for several physiological traits. Pasto showed the highest reduction in SLA and transpiration, the lowest concentration $\mathrm{Na}^{+}$and $\mathrm{Cl}^{-}$in young leaves, the highest concentration of $\mathrm{K}^{+}$in young leaves, and the lowest reduction in nitrate concentration in young leaves throughout the growing season. In addition, flowering, seed filling and seed setting times were delayed for Pasto (not shown). Its growth was more reduced than the other varieties, but the plants still appeared to be healthy, which is supported by the highest RWC, the highest NAR increase and an increase in chlorophyll content throughout the season. We speculate that Pasto employed a "survival" strategy with a more reduced growth rate, transpiration rate and higher rate of exclusion of $\mathrm{Na}^{+}$. These adaptations allowed Pasto and Pasto-like varieties to survive longer, but at the trade-off of the very high reduction in growth rate and seed production.

\section{Acknowledgments}

The authors gratefully acknowledge the expert assistance of Geurt Versteeg and Andre Maassen in the greenhouse experiments. Viviana Jaramillo is financially supported by a scholarship from the Secretariat for Higher Education, Science, Technology and Innovation of the Republic of Ecuador (Contract Number 2015AR2Q8878). 


\section{Supplementary material}

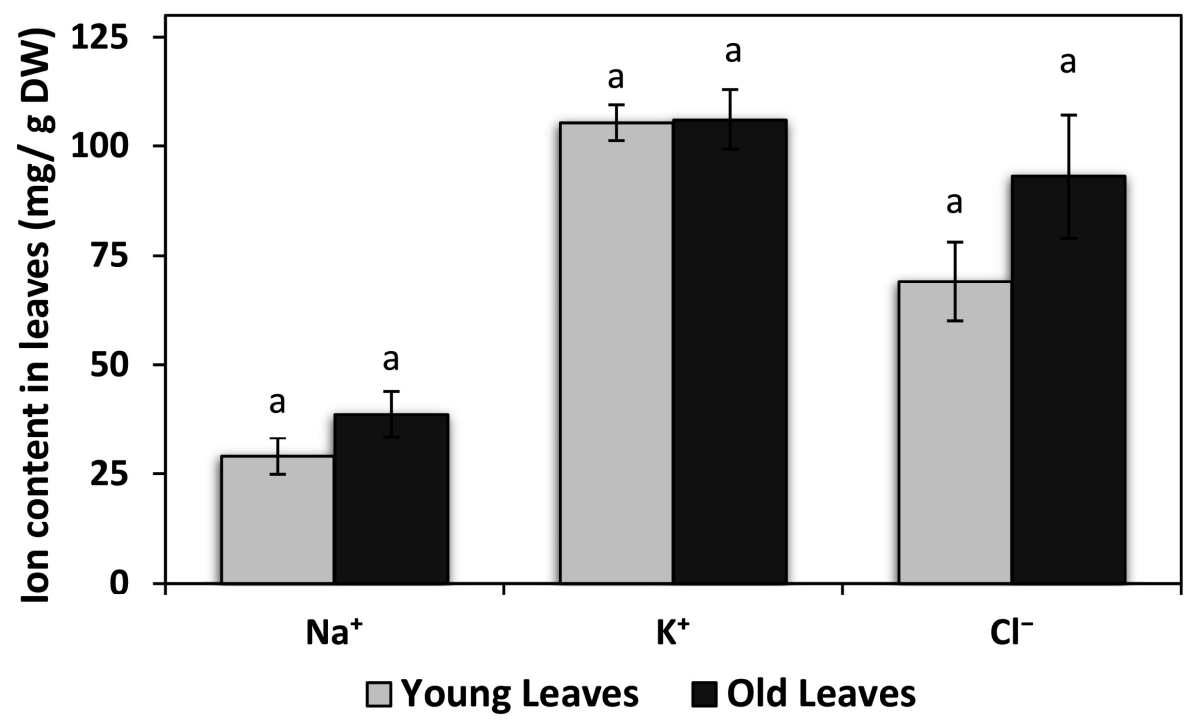

Figure S1. Comparison of ion contents between young and old leaves of quinoa grown in Experiment 3 at $400 \mathrm{mM} \mathrm{NaCl} 12$ weeks after sowing, 7 weeks after start of the salt treatment. Means of three varieties (Jessie, Pasto, selRiobamba) and 3 replicates per variety. Error bars indicate SE of individual means. Statistically significant differences $(p \leq 0.05)$ between young and old leaves for each ion are shown with different letters. 


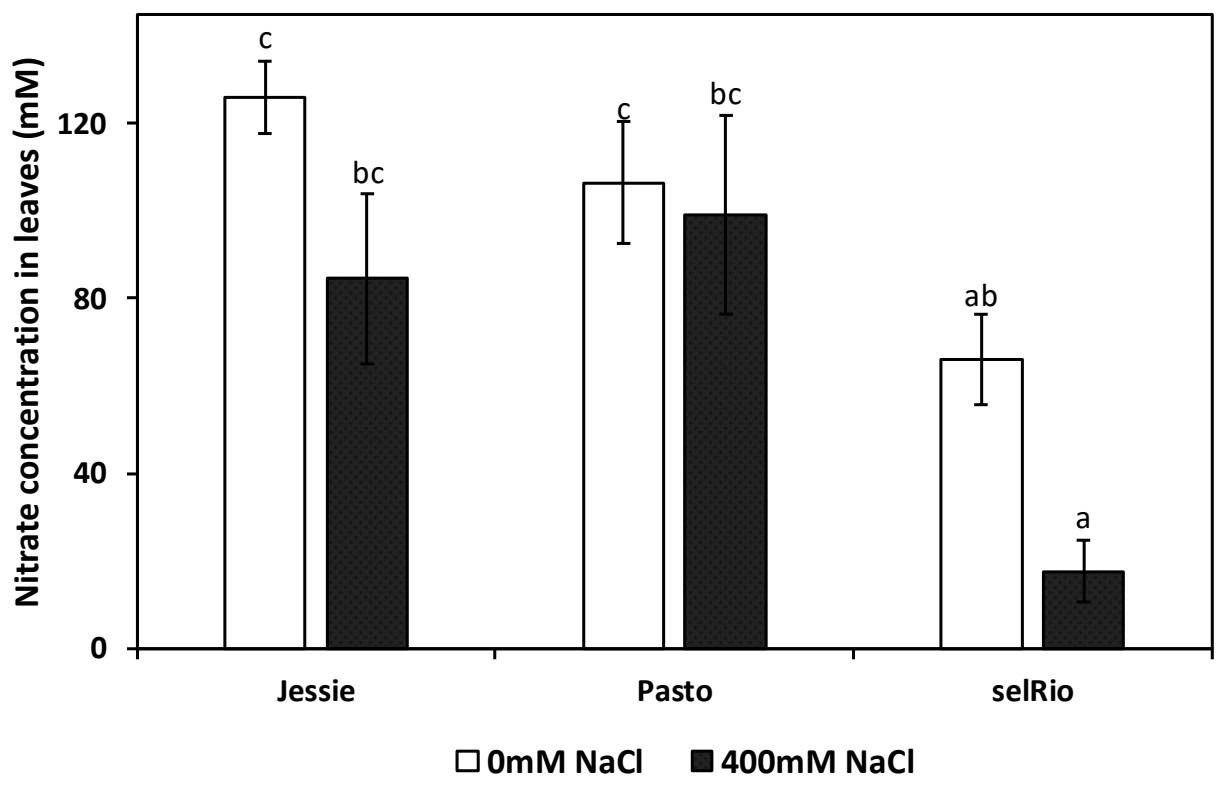

Figure S2. Nitrate concentration in young leaves of quinoa growing in Experiment 3 at $400 \mathrm{mM} \mathrm{NaCl} 12$ weeks after sowing, 7 weeks after start of the salt treatment. Means of 3 plants. Error bars indicate SE of individual means. Statistically significant differences $(p \leq 0.05)$ between any variety and salt treatment combination are shown with different letters. 


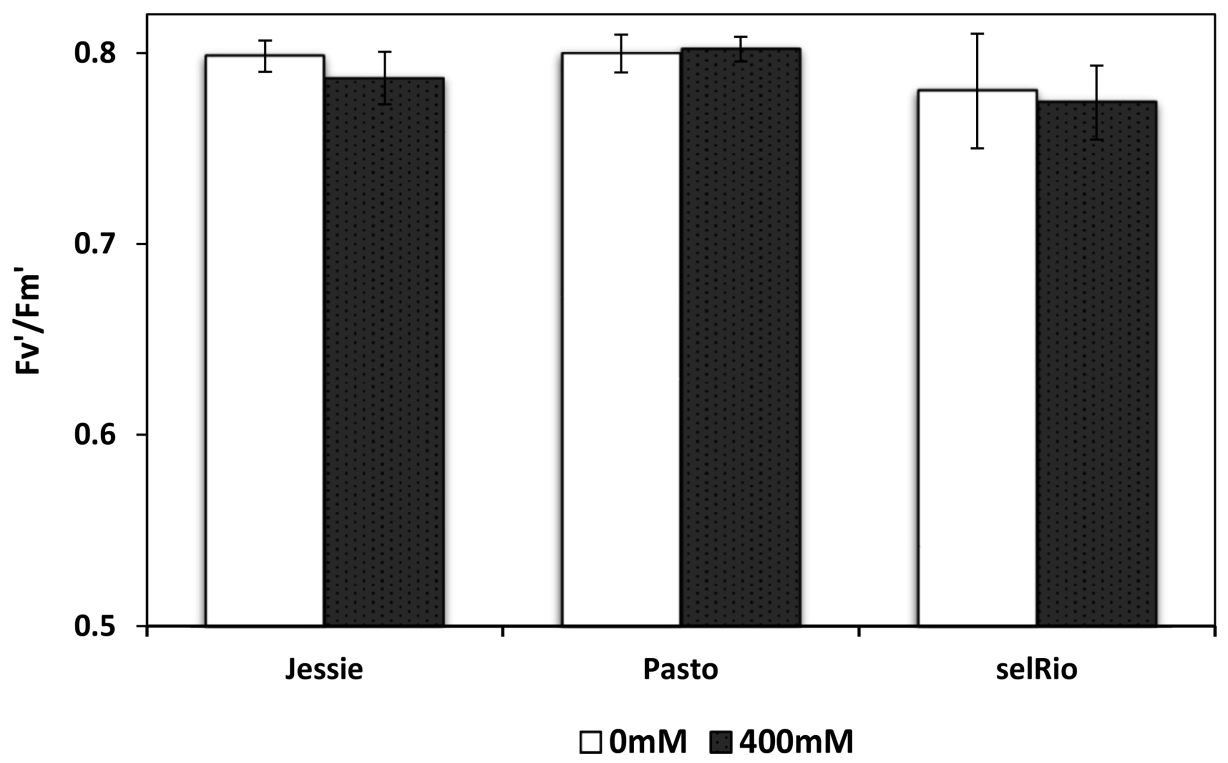

Figure S3. Maximum quantum yield of PSII ( $F v / F m)$ after dark adaptation measured in young leaves of quinoa plants growing in Experiment 3 at $400 \mathrm{mM} \mathrm{NaCl} 12$ weeks after sowing, 7 weeks after start of the salt treatment. Means of 3 plants. Error bars indicate SE of individual means. Statistically significant differences $(p \leq 0.05)$ between any variety and salt treatment combination are shown with different letters. 


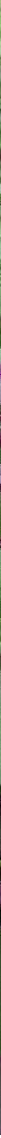

\section{Chapter 3}

Cell wall modifications of Chenopodium quinoa in response to severe salt stress

Viviana Jaramillo Roman, Agata Gulisano, Edoardo Vergnano, Behzad Rashidi, Annemarie Dechesne, Luisa M. Trindade, Richard G.F. Visser, C. Gerard van der Linden, Eibertus N. van Loo 
Plant cell walls are complex structures in terms of composition, structure and function. Here, we studied the effects of high soil salinity on the biochemical composition of cell walls in stems and leaves of the salt tolerant species Chenopodium quinoa. Chemical analysis of cell walls showed that monosaccharides composing pectin are increased in quinoa cell walls under saline conditions with the highest increase in arabinose (160\% increase in stems and $60 \%$ increase in leaves), while the contents of glucose and lignin were significantly reduced. The inorganic constituents of the cell wall also varied between control and stress conditions. $\left[\mathrm{Ca}^{2+}\right]$ was significantly reduced by $30 \%$ and $65 \%$ under salinity in the stem and leaf-cell wall, respectively. In contrast, [ $\left.\mathrm{Na}^{+}\right]$was increased under salinity by $140 \%$ and $70 \%$ in cell walls of stems and leaves, respectively. We argue that this altered cell wall composition reflects a functional adaptation of this species to high salinity. The possible displacement of pectin-bound $\mathrm{Ca}^{2+}$ by $\mathrm{Na}^{+}$ disrupts pectin cross-linking causing loosening of the cell wall, which may be compensated by increased branching of arabinoserich polymers. The higher abundance of pectic branching polymers would increase the flexibility and hydration of the cell wall improving the adaptation to changes in osmotic potential imposed by salinity. The changes in the cell wall composition of salt-stressed quinoa described here furthers our as yet limited understanding of the role of cell wall remodelling in salt stress tolerance. 


\section{Introduction}

Plant growth is the result of cell division, cellular growth and expansion. Growth at a cellular level is a coordinated balance between turgor pressure -the physical force needed to drive cell enlargement- and the extensibility of the cell wall (Cosgrove 2015). Plant cell walls are dynamic structures that surround all plant cells and are intrinsically involved in their growth, morphology, as well as their interaction with the environment, including responding to environmental stresses such as salinity. Soil salinity causes a reduction in the external water potential and, consequently, a decrease of turgor pressure in the cells. Plant cells adapt to these changes through osmotic adjustment, reaching a new equilibrium with the external water potential. Eventually, growth is restored, though at a lesser extent (Hasegawa et al. 2000). Since growth is modulated by cell wall synthesis and expansion, changes in the biochemical and biomechanical properties of cell walls are important plant cell responses to turgor changes upon salt stress. Several studies have examined the effect of salt stress on the cell wall, mainly in roots (Byrt et al. 2018), while reports on the effect of salinity on leaf cell walls are scarce (Tenhaken 2015). Most of the existing ideas on how the cell wall responds to salt stress have been inferred from transcriptomic and proteomic analysis of expression of the genes and enzymes that synthesize and modify the cell wall, but salt-induced changes in its chemical composition and structure still need to be established (Rui and Dinneny 2020).

Cell walls are matrices with variable arrangements of polysaccharides, glycoproteins, proteins and enzymes, depending on the plant species, cell type, location, developmental stage and environmental conditions (Wolf et al. 2012). Primary cell walls of dicots consist of a cellulosic network embedded in a matrix of mainly xyloglucans and pectins; they lack lignification and are deposited during cell growth. Cells that have stopped enlarging mature by depositing secondary walls that increase the strength of the cell and reduce flexibility. Secondary walls are mostly composed of cellulose, hemicelluloses and lignin (Doblin et al. 2010). In most plant species, the most abundant component of the cell wall is cellulose; microfibrils of glucose that form the extracellular network and provide mechanical strength to the cell wall (Cosgrove 2005). Hemicelluloses are a group of polysaccharides with a backbone of glucose, mannose or xylose. The most important role of hemicelluloses is the strengthening of the cell wall by cross-linking cellulose microfibrils and lignin in the secondary cell wall (Scheller and Ulvskov 2010). Lignins are complex phenolic polymers that can provide strength, rigidity and hydrophobicity to plant cell walls (Kumar et al. 2015). Pectins are a highly heterogeneous group of acidic 
polysaccharides that contribute to the mechanical strength, porosity, extension, adhesion and stiffness of the cell wall (Burton et al. 2010). Galacturonic acid (GalA) is the main backbone component of the three dominant pectin types: homogalacturonan (HG), rhamnogalacturonan-I (RG-I) and rhamnogalacturonan-II (RG-II). HG is the main pectin component of the cell wall of land plants, since it stiffens the primary cell wall through the formation of $\mathrm{Ca}^{2+}$ bridges (Peaucelle et al. 2012). RG-I is a highly diverse polymer. Its backbone is formed by the disaccharide rhamnose-GalA, and $20-80 \%$ of rhamnose residues are substituted with branching side chains, mostly composed of arabinose (arabinans) and galactose (galactans) (Caffall and Mohnen 2009). The reason for the high diversity of RG-I is not totally understood but it suggests diverse functional specialization (Caffall and Mohnen 2009). On the contrary, RG-II is a highly conserved type of pectin that has the capacity to dimerize through borate ester links (Willats et al. 2001). Analysing the compositional changes in the different cell wall constituents is an important step towards the understanding of the cell wall remodelling in response to abiotic stress.

The goal of the research described in this paper was to examine the effect of salt stress in the cell wall composition of stems and leaves of Chenopodium quinoa. Quinoa is a facultative halophyte that grows and thrives under non-saline conditions, but also survives under severe saline conditions. Several physiological strategies utilized by quinoa to tolerate prolonged exposure to soil salinity have been described (Jaramillo Roman et al. 2020). We describe in this chapter the changes in the chemical composition of quinoa cell walls as a consequence of salt stress, and provide insight into the remodelling of the cell wall, which might play a role in the ability of this species to survive severe salt stress.

\section{Materials and Methods}

\subsection{Experimental conditions}

Three sweet European commercial quinoa varieties were used in this study: Jessie, Pasto and selRiobamba (a line selected from Riobamba (Riobamba has still some residual heterozygosity)). The impact of salt stress on the growth and several physiological traits of these varieties was previously described (Jaramillo Roman et al. 2020). The plants were grown in $3 \mathrm{~L}$ pots using vermiculite No. 3 as substrate. The plants were irrigated with half concentrated Hoagland's nutrient solution. Salt stress treatment started five weeks after sowing. Salt was applied by incremental increases of $75 \mathrm{mM} \mathrm{NaCl}$ every two days, until a final concentration of $400 \mathrm{mM} \mathrm{NaCl}$ was reached, and the salt concentration in the substrate was continuously monitored 
during the whole experiment. Three replicates per variety and salt treatment were harvested 16 weeks after sowing (11 weeks after start of the salt treatment) and samples of young leaves and stems were used for cell wall analysis. This trial was conducted at the Unifarm greenhouse facilities of Wageningen University \& Research, The Netherlands. The greenhouse air humidity was set to a minimum of $80 \%$. When the incoming shortwave radiation was below $200 \mathrm{Wm}^{-2}$, additional lighting was supplied $\left(100 \mathrm{Wm}^{-2}\right)$. Light irradiance, air temperature, water content and electrical conductivity $(E C)$ in the pots were monitored via wireless sensors (Flower Power ${ }^{\mathrm{TM}}$ ).

\subsection{Cell wall extraction}

Leaves and stems were air-dried at $60{ }^{\circ} \mathrm{C}$ until samples reached stable weights, and afterwards grinded to a $1 \mathrm{~mm}$ particle size. Cell walls of stems were isolated following an alcohol-insoluble residue (AIR) method previously described (Petit et al. 2019). In brief, $1 \mathrm{~g}$ of dried-grinded stem was incubated in $35 \mathrm{~mL}$ of 80 $\%$ ethanol $(\mathrm{v} / \mathrm{v})$ on ice for $30 \mathrm{~min}$. The samples were then centrifuged for $5 \mathrm{~min}$ at $10000 \mathrm{~g}$ and $4{ }^{\circ} \mathrm{C}$ (ultracentrifuge Beckman Coulter J2-21M, rotor F14BA- 14x50cy, Indianapolis, USA) and the supernatant was discarded. This extraction step was repeated three times. Cell wall extraction continued with the removal of lipids and other intracellular components. Samples were incubated in $35 \mathrm{~mL}$ of acetone for 10 $\mathrm{min}$ at room temperature and centrifuged for $5 \mathrm{~min}$ at $10000 \mathrm{~g}$ at room temperature. The supernatant was discarded and the samples went through an additional washing step of 10 min incubation in $35 \mathrm{~mL}$ of absolute methanol followed by centrifugation for $5 \mathrm{~min}$ at $10000 \mathrm{~g}$ at room temperature. The washed residual pellets were dried using a RapidVap Vacuum Dry Evaporation System (Labconco, Missouri, USA). The protocol continued with the removal of residual starch from the AIR-extracted cell wall. The pellets were incubated in $6 \mathrm{~mL}$ of $10 \mathrm{mM}$ Tris-maleate buffer for $30 \mathrm{~min}$ and then agitated at $100^{\circ} \mathrm{C}$ for $5 \mathrm{~min}$ to promote the gelatinization of starch granules. The samples were equilibrated to $40^{\circ} \mathrm{C}$ and subjected to two rounds of $\alpha$-amylase digestion (Megazyme, Bray, Ireland). In the first round, samples were incubated for $1 \mathrm{~h}$ at $40{ }^{\circ} \mathrm{C}$ with an enzyme concentration of $2 \mathrm{U} \mathrm{mg}^{-1}$, and in the second-round samples were incubated for $30 \mathrm{~min}$ at $40{ }^{\circ} \mathrm{C}$ with half of the concentration of the enzyme. The enzyme was inactivated by adding $36 \mathrm{~mL}$ cold ethanol and incubation at $-20{ }^{\circ} \mathrm{C}$ for 1 hour. Finally, pellets were washed three times with cold absolute ethanol, centrifuging between each wash at $1500 \mathrm{~g}$ at room temperature. The final light brown-coloured pellets were dried using a RapidVap and represent the total AIR-extracted stem cell wall. 
Cell walls of young leaves were isolated with an AIR method with additional chloroform and SDS washing steps to remove residual chlorophyll and other intracellular components. This protocol was optimized from (Abedon et al. 2006). One gram of dried-grinded leaves was initially incubated with $40 \mathrm{~mL}$ of Tris-acetate buffer in an ultrasonic bath (VWR International, Pennsylvania, USA) for $15 \mathrm{~min}$ and centrifuged ( $30 \mathrm{~min}, 10000 \mathrm{~g}, 4{ }^{\circ} \mathrm{C}$ ). The supernatant was discarded and the incubation was repeated two times. The protocol continued with the extraction of the cell wall using $80 \%$ ethanol. Samples were incubated for $15 \mathrm{~min}$ in $35 \mathrm{~mL} 80 \%$ ethanol in an ultrasonic bath four times, with a centrifugation step (7 min, $10000 \mathrm{~g}, 4$ ${ }^{\circ} \mathrm{C}$ ) between each incubation. After the fourth time, the pellets were incubated in 35 $\mathrm{mL}$ acetone in the ultrasonic bath for $15 \mathrm{~min}$, centrifuged $\left(10 \mathrm{~min}, 10000 \mathrm{~g}, 4{ }^{\circ} \mathrm{C}\right.$ ), and the supernatant was discarded. The pellets were then washed with a chloroform/methanol $(2: 1)$ solution, vortexed thoroughly, placed in the ultrasonic bath for $15 \mathrm{~min}$, centrifuged (10 $\mathrm{min}, 10000 \mathrm{~g}, 4{ }^{\circ} \mathrm{C}$ ), and the supernatant was discarded. Afterwards, the pellets were incubated in $30 \mathrm{~mL} \mathrm{SDS} 1 \%$ for $1 \mathrm{~h}$ at 100 ${ }^{\circ} \mathrm{C}$, centrifuged (10 min, $10000 \mathrm{~g}, 4{ }^{\circ} \mathrm{C}$ ), and incubated in $0.5 \%$ SDS half for another hour. Finally, the samples were incubated in $35 \mathrm{~mL}$ of acetone for $10 \mathrm{~min}$ at room temperature and centrifuged for $5 \mathrm{~min}$ at $10000 \mathrm{~g}$ at room temperature. The final white pellets were dried using a RapidVap and correspond to the total AIR-extracted leaf cell wall.

\subsection{Monosaccharide composition of the extracted cell wall}

A two-step sulfuric acid hydrolysis described by Petit et al. (2019) was applied to characterize the monosaccharide composition of the cell wall of leaves and stems. In brief, $20 \mathrm{mg}$ of AIR-extracted cell wall was incubated in $1 \mathrm{~mL}$ of $72 \%$ (v/v) $\mathrm{H}_{2} \mathrm{SO}_{4}$ and stirred for $1 \mathrm{~h}$ at $30{ }^{\circ} \mathrm{C}$. The acid concentration was subsequently diluted to $6 \%(\mathrm{v} / \mathrm{v})$ and the incubation continued for $1 \mathrm{~h}$ at $121^{\circ} \mathrm{C}$ in an autoclave (Tuttnauer 3850EL, Breda, Netherlands). Following the hydrolysis, samples were cooled down and filtered using a $0.45 \mu \mathrm{m}$ PTFE filter. The filtered samples were again 10x diluted for the monosaccharide analysis by High Performance Anion Exchange Chromatography (HPAEC) on a Dionex® ICS5000+ DC (Detector/Chromatography Compartment) equipped with a Dionex® CarboPac PA1 $(2 \times 50 \mathrm{~mm})$ (Dionex/Thermo Fisher Scientific, Massachusetts, USA) as described (Rashidi and Trindade 2018). The retention time of each sugar was determined after a single injection into the system, and the quantification was done against a calibration curve of several dilutions of commercial monosaccharides. The sugar concentration in the cell walls was further corrected based on sugar recovery standards, used to quantify the amount of monosaccharides that might have been 
degraded during the sulphuric acid hydrolysis (Petit et al. 2019). The content of each monosaccharide in the cell wall was expressed as mg monosaccharide per $\mathrm{g}$ dry AIR-extracted cell wall.

\subsection{Lignin quantification in extracted cell walls}

The content of lignin in the cell wall of leaves and stems was determined based on the Klason Lignin estimation described by Petit et al. (2019). Klason lignin is defined as the insoluble residue of the cell wall after sulphuric acid hydrolysis (Vermerris and Nicholson 2006). After the two-step $\mathrm{H}_{2} \mathrm{SO}_{4}$ hydrolysis, the precipitates were vacuum-filtered in a pre-weighed AP40-47 mm diameter glass fibre filter (Millipore Sigma, Massachusetts, USA). The residues were dried at $103^{\circ} \mathrm{C}$ for 12 hours and these represent the dry Klason lignin of the sample.

\subsection{Inorganic components of extracted cell wall}

The ion contents in young leaves, stems and in the AIR-extracted cell walls from young leaves and stems were measured using lon Chromatography (IC) system 850 Professional (Metrohm Switzerland). Briefly, $25 \mathrm{mg}$ of dry sample (extracted cell wall or tissue) were turned into ashes in a furnace at $550{ }^{\circ} \mathrm{C}$ for $5 \mathrm{~h}$. Ash content of each sample was expressed as the weight ratio of ashes and dry sample. Ashes were dissolved in $1 \mathrm{~mL} 3 \mathrm{M}$ formic acid at constant shaking at 500 rpm for $15 \mathrm{~min}$ at $99^{\circ} \mathrm{C}$. The dissolved ashes were 400x diluted with Milli-Q® water and injected onto the IC column. Ion contents were calculated as the ion weight per unit of dry weight ( $\mathrm{mg}$ ion $\mathrm{g}^{-1}$ dry mass).

\section{Results}

\subsection{Growth and biomass allocation under salinity}

Salt stress reduced the growth of quinoa plants. A detailed analysis of the impact of the salt treatment of $400 \mathrm{mM} \mathrm{NaCl}$ on the physiology and growth of these European quinoa varieties was reported by us previously (Jaramillo Roman et al. 2020). In short, after seven weeks of salt treatment (11 weeks after sowing) total biomass decreased on average by $70 \%$ compared to control conditions $(0 \mathrm{mM} \mathrm{NaCl})$ (Figure 1A). Biomass allocation was also influenced by salinity. More biomass was allocated to roots, less to heads, while the proportions of leaves and stems were not significantly altered by the treatment (Figure 1B). Even though the plant biomass was significantly reduced by salinity, the varieties displayed remarkable salt tolerance, being able to survive, grow, remain green and produce seeds despite the high salt stress imposed. 

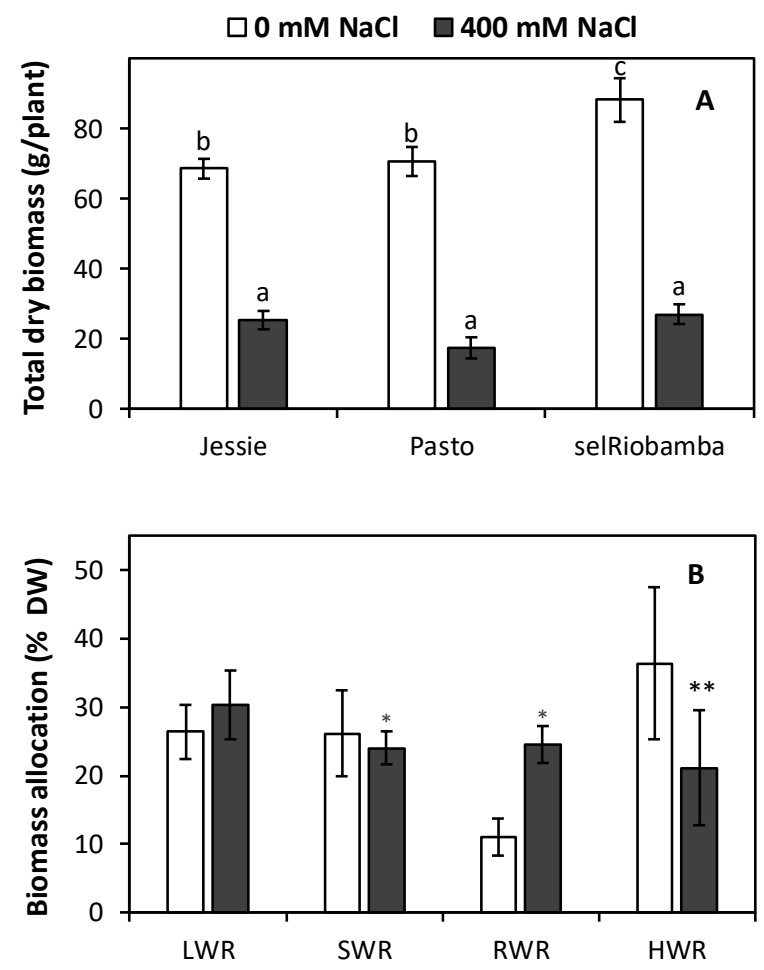

Figure 1. Biomass traits of quinoa growing at $400 \mathrm{mM} \mathrm{NaCl}$. A) Total dry biomass weight 11 weeks after sowing, 6 weeks after salt application. Error bars indicate SE of individual means. Statistically significant differences $(p \leq 0.05)$ between any variety and salt treatment combination are shown with different letters. B) Biomass allocation as a percentage of the total weight of single plants. Means of three varieties (Jessie, Pasto, selRiobamba) and 3 replicates per variety. Error bars indicate SE of individual means. Asterisks denote statistically differences $\left({ }^{*} p \leq 0.05,{ }^{* *} p \leq 0.01,{ }^{* * *} p \leq 0.001\right.$ ) between the salt treatment compared to the control. Abbreviations: LWR (leaf weight ratio), SWR (stem weight ratio), RWR (root weight ratio), HWR (head weight ratio).

\subsection{Cell wall composition under salinity}

The cell wall composition with respect to the main constituents was different between stems and leaves and was altered by salt stress in both tissues (Figure 2AB). Salt stress did not significantly affect the amount of extracted cell wall in stems, and $83 \%$ of the total AIR-extracted cell wall composition could be identified by the different biochemical tools used in this study. The main components of quinoa stem cell walls were polysaccharides, which accounted for $79 \%$ of the total cell wall under control conditions and were slightly increased to $82 \%$ at $400 \mathrm{mM} \mathrm{NaCl}$. Klason lignin represented $17.5 \%$ of the total cell wall and was reduced to $13.5 \%$ at high salinity. The inorganic component of the stems cell walls was not significantly affected by the salt treatment. 
Only $60 \%$ of the total AIR-extracted cell wall from leaf tissue could be identified by the analytical procedures used in our research under both control and salt treatment. No significant differences were found between treatments in the amount of extracted cell wall. Of the total cell wall composition in leaves, the polysaccharide content was $62 \%$, and this significantly increased to $72 \%$ under high salinity. The content of lignin in the cell wall of leaves significantly decreased from $25 \%$ under control conditions to $20 \%$ under salinity and the inorganic materials from $12 \%$ to $8 \%$.

Especially in the leaves, the polysaccharide content of the cell wall increased due to salt stress. In order to see whether this change might affect the structural properties of the cell wall, we analysed the monosaccharides constituting the main backbone polysaccharides of the cell wall: pectin, cellulose and hemicellulose.
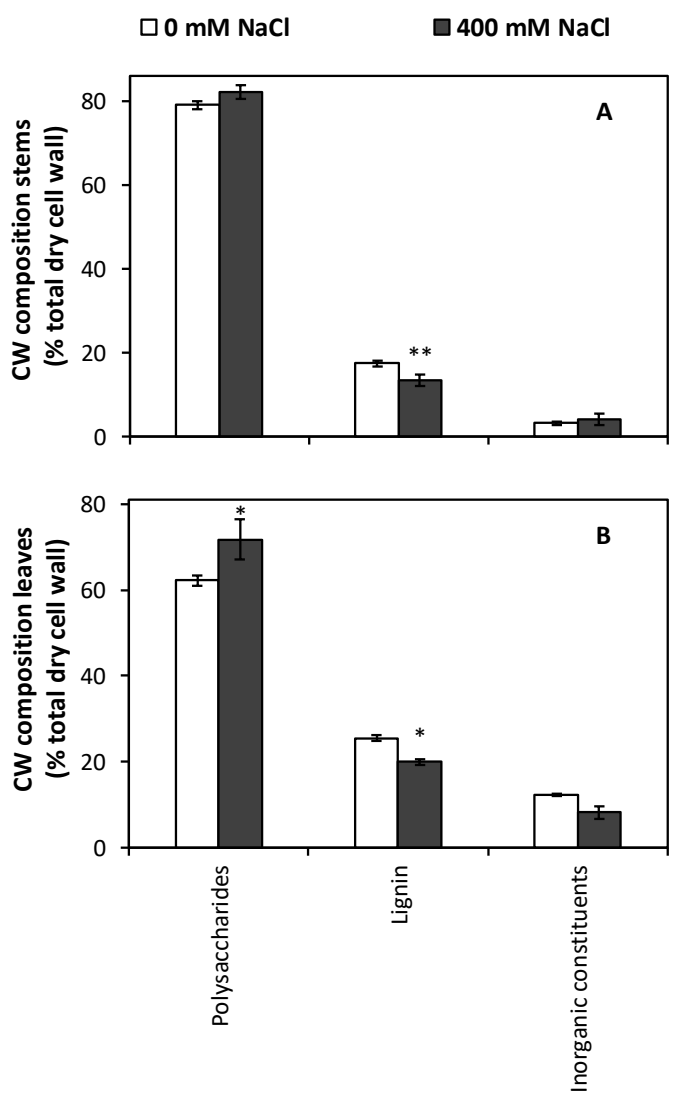

Figure 2. Cell wall composition of quinoa leaves and stems under control and high salinity. A) Stems. B) Leaves. Means of three varieties (Jessie, Pasto, selRiobamba) and 3 replicates per variety. Error bars indicate SE of individual means. Asterisks denote statistically differences $\left({ }^{*} p \leq 0.05,{ }^{* *} p \leq 0.01,{ }^{* * *}\right.$ $p \leq 0.001$ ) between the salt treatment compared to the control. 


\subsection{Monosaccharide composition of stem and leaf cell walls under severe salinity}

The monosaccharide composition of stems and leaves cell wall fractions was affected by salt stress (Figure 3A-B). Glucose, which is the main building block for cellulose, was the most abundant sugar in the stem cell walls under control conditions and accounted for $50 \%$ of the total sugar composition, followed by xylose (27\%) (the main component of hemicellulose), and the pectin components galacturonic acid, mannose and arabinose (Figure 3A). The fraction of each of the monosaccharides in the cell wall, except xylose, was affected by the salt treatment and the extent of those differences varied between genotypes (Figure 4A-D). Galacturonic acid, arabinose, galactose and rhamnose were significantly increased by salt stress in stems of all the varieties. Arabinose increased by an average $163 \%$, galactose by $63 \%$ and galacturonic acid by $26 \%$. Glucose and mannose contents were reduced under salinity by $13 \%$ and $19 \%$, respectively, with the bigger reductions measured for the variety Pasto.

The monosaccharide composition of the leaf cell walls was different compared to stems. Although glucose was the most abundant sugar in both stem and leaf cell walls, this monosaccharide accounted for only $40 \%$ of the total sugar composition in leaf cell walls. Galacturonic acid, arabinose, galactose and rhamnose were also well-represented, suggesting a high amount of pectin in the leaf cell-wall. (Figure 3B). The sugar composition of leaf cell walls was also affected by the salt treatment. Galacturonic acid was the second most abundant sugar after glucose under control conditions, but under high salinity arabinose content increased from $14 \%$ to $23 \%$ to become the second most abundant sugar in the leaf cell wall of saltstressed leaves. The other pectic-related monosaccharides (galacturonic acid, galactose and rhamnose) were strongly increased in the variety Pasto, but the increase was not significant in the other two varieties (Jessie and selRiobamba) (Figure 4G-H). Similarly, the content of glucose, xylose and mannose was increased by salinity in Pasto, while the concentration of these monosaccharides in Jessie and selRiobamba remained the same (Figure 4E-F). 

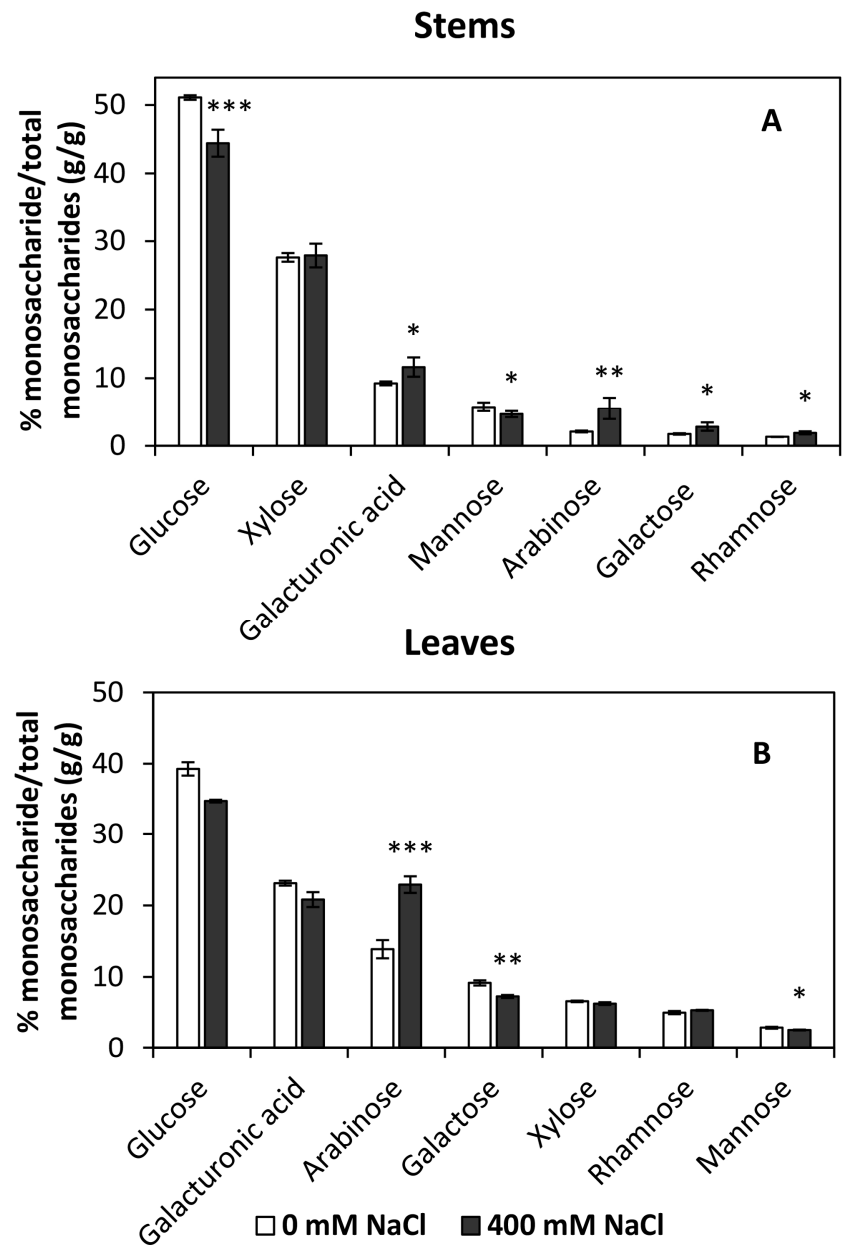

Figure 3. Relative contribution of monosaccharides to the total monosaccharide content of the cell wall. A) Stems. B) Leaves. Means of three varieties (Jessie, Pasto, selRiobamba) and 3 replicates per variety. Error bars indicate SE of individual means. Asterisks denote statistically differences $\left({ }^{*} p \leq 0.05,{ }^{* *} p \leq 0.01\right.$, ${ }^{* * \star} p \leq 0.001$ ) between the salt treatment compared to the control. 

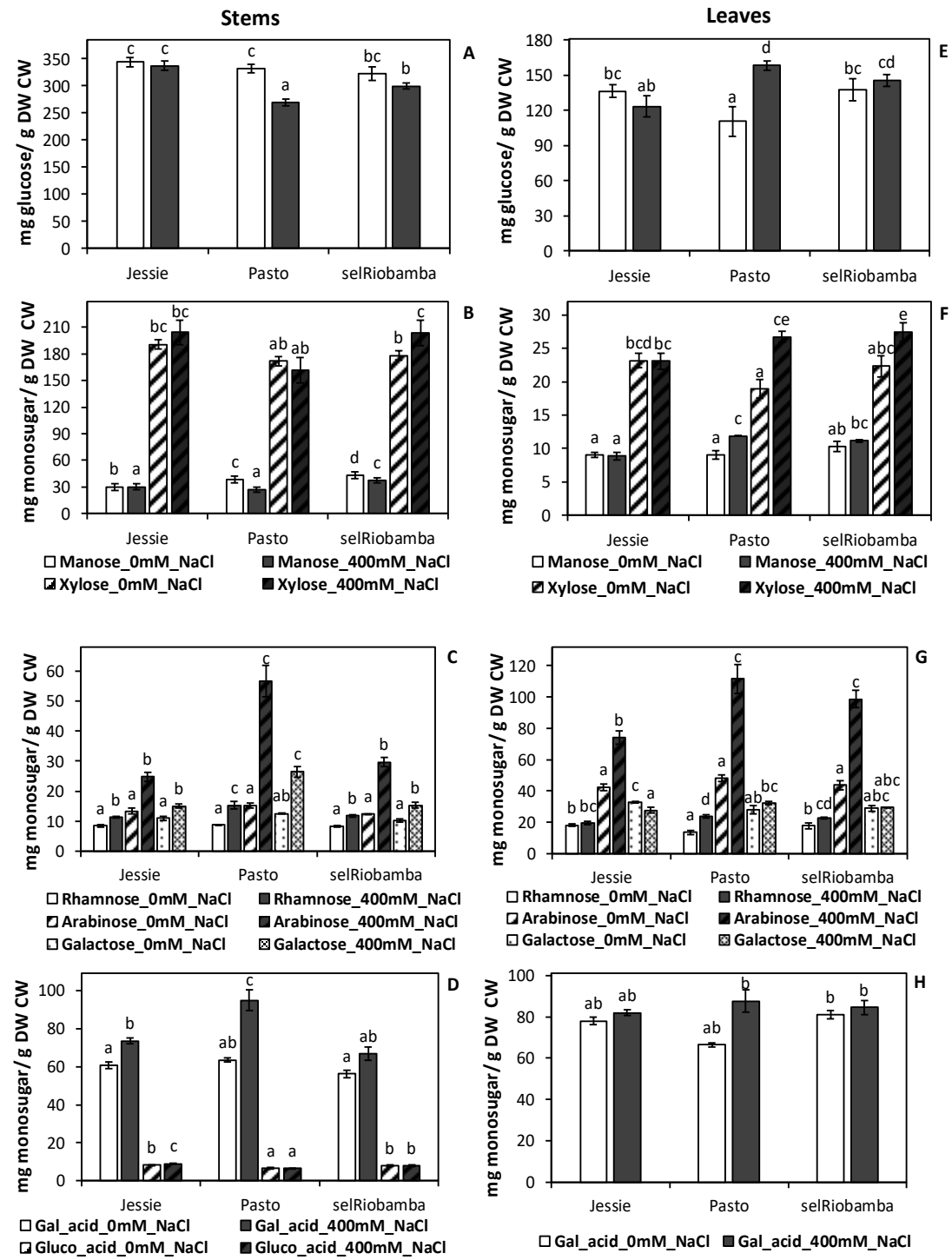

Figure 4. Monosaccharide composition of quinoa cell walls in stems and leaves relative to the total cell wall content. A) Glucose content in stems. B) monosaccharides related with hemicellulose (mannose and xylose) content in stems. C) Monosaccharides related with pectin (rhamnose, arabinose, galactose) content in stems. D) Uronic acid content in stems. E) Glucose content in leaves. F) monosaccharides related with hemicellulose (mannose and xylose) content in leaves. G) Monosaccharides related with pectin (rhamnose, arabinose, galactose) content in leaves. $H$ ) Uronic acid content in leaves. Means of 3 plants. Error bars indicate SE of individual means. Statistically significant differences $(p \leq 0.05)$ between any variety and salt treatment combination for each monosaccharide are shown with different letters. 


\subsection{Salinity effects on inorganic cell wall components}

Inorganic ions like $\mathrm{Ca}^{2+}$ are important elements of the cell wall. Salinity influences ion homeostasis in the plant, and might affect the ion composition of the cell wall. We measured the ion content in the AIR-extracted cell wall from stems and leaves to understand salinity-induced changes and their association with structural remodelling of the cell wall. Figure 5A-L compares the ion concentrations measured in the extracted cell walls from leaves and stems. $\mathrm{Ca}^{2+}$ was the predominant cation in the cell wall of leaves and stems and its concentration was significantly higher in the cell wall than in the entire tissue. Under control conditions, $\mathrm{Ca}^{2+}$ was $38 \%$ and $150 \%$ higher in the cell wall than in stems and leaves, respectively. $\left[\mathrm{Ca}^{2+}\right]$ decreased due to salinity in the cell walls of stems and leaves, but not for stem cell walls of Pasto. The $\left[\mathrm{Mg}^{2+}\right]$ in the cell wall of stems was similar to the tissue concentration. Pasto had a significant increase in the $\left[\mathrm{Mg}^{2+}\right]$ both in the stem tissue and stem cell wall under high salinity. For the other two varieties, no significant differences were found between the leaves and stems, between the leaf and stem cell walls, or between the treatments.

The measured concentrations of $\mathrm{Mg}^{2+}$ in leaves and of $\mathrm{Na}^{+}$and $\mathrm{K}^{+}$in both tissues were considerably lower in the cell walls than in the tissues. The cell wall extraction approach used in this study includes several washing steps, and only the ions that constitute structural components of cell wall polymers will be retained in the extracted cell wall, as is the case for $\mathrm{Ca}^{2+}$. However, other ions that are present in the cell wall in vivo may be lost during the extraction procedure. Therefore, the function of the apoplast as a potential transient reservoir for ions such as $\mathrm{Na}^{+}$and possibly $\mathrm{K}^{+}$cannot be examined with our current methodology. Nevertheless, the $\left[\mathrm{Na}^{+}\right]$measured in the extracted cell wall from salinity-stressed plants was significantly increased by $90 \%$ in leaves and $137 \%$ in stems. From the measured inorganic anions, only phosphate and sulphate were detected in the stem cell walls and only sulphate in the leaf cell walls. The content of phosphate and sulphate in stem cell walls was not affected by soil salinity, except for Pasto, which showed a significantly increase in the concentration of both anions. Sulphate increased by 40 $\%$ in the leaf cell walls of Pasto. This variety showed also the highest increase in cations, which may indicate that this increase served to prevent charge imbalances in the Pasto cell walls. 

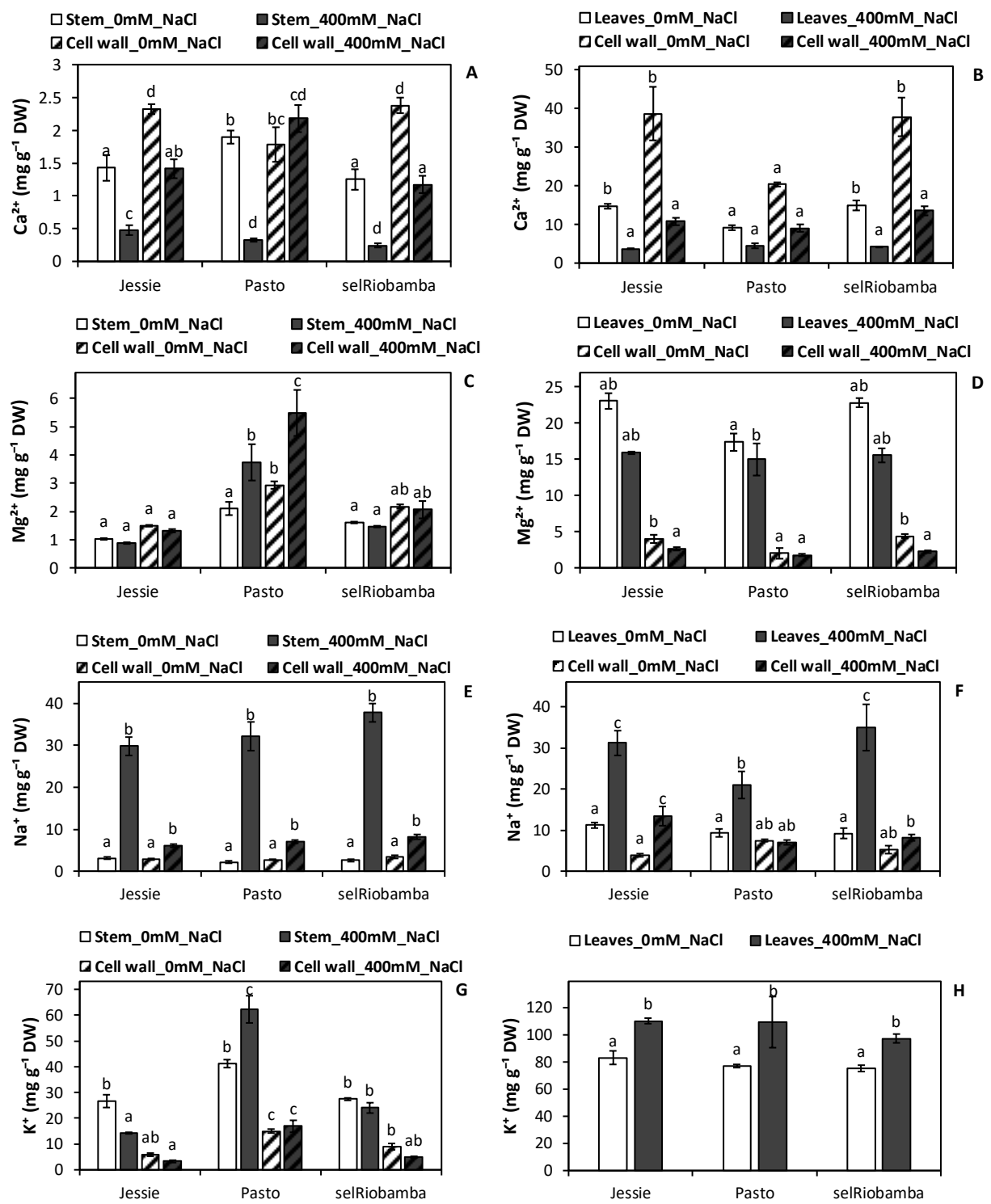

\section{$\square$ Leaves_0mM_NaCl $\square$ Leaves_400mM_NaCl}

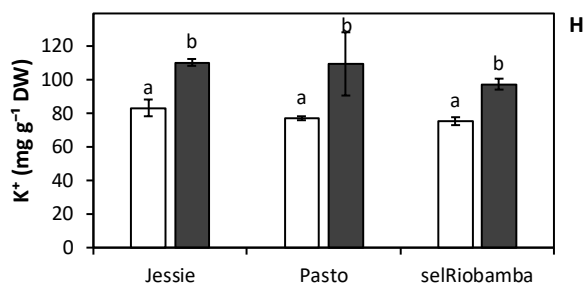

Figure 5. Ion contents in leaves and stems, and cell walls of leaves and stems of quinoa plants exposed to high salinity expressed as $\mathrm{mg}$ ion per $\mathrm{g}$ dry weight of each respective tissue (stems/leaves/cell walls). A) $\left[\mathrm{Ca}^{2+}\right]$ in stems and stem-cell walls. B) $\left[\mathrm{Ca}^{2+}\right]$ in leaves and leaf-cell walls. C) $\left[\mathrm{Mg}^{2+}\right]$ in stems and stemcell walls. D) $\left[\mathrm{Mg}^{2+}\right]$ in leaves and leaf-cell walls. E) $\left[\mathrm{Na}^{+}\right]$in stems and stem-cell walls. $F$ ) $\left[\mathrm{Na}^{+}\right]$in leaves and leaf-cell walls. G) $\left[\mathrm{K}^{+}\right]$in stems and stem-cell walls. $\left.\mathrm{H}\right)\left[\mathrm{K}^{+}\right]$in leaves and leaf-cell walls. I) $\left[\mathrm{PO}_{4}{ }^{3}\right]$ in stems and stem-cell walls. J) $\left[\mathrm{PO}_{4}{ }^{3}\right]$ in leaves and leaf-cell walls. $\left.\mathrm{K}\right)\left[\mathrm{SO}_{4}{ }^{2}\right]$ in stems and stem-cell walls. $\mathrm{L})\left[\mathrm{SO}_{4}{ }^{2}\right]$ in leaves and leaf-cell walls. Means of 3 plants. Error bars indicate SE of individual means. Statistically significant differences $(p \leq 0.05)$ between any variety and salt treatment combination for each tissue are shown with different letters. 

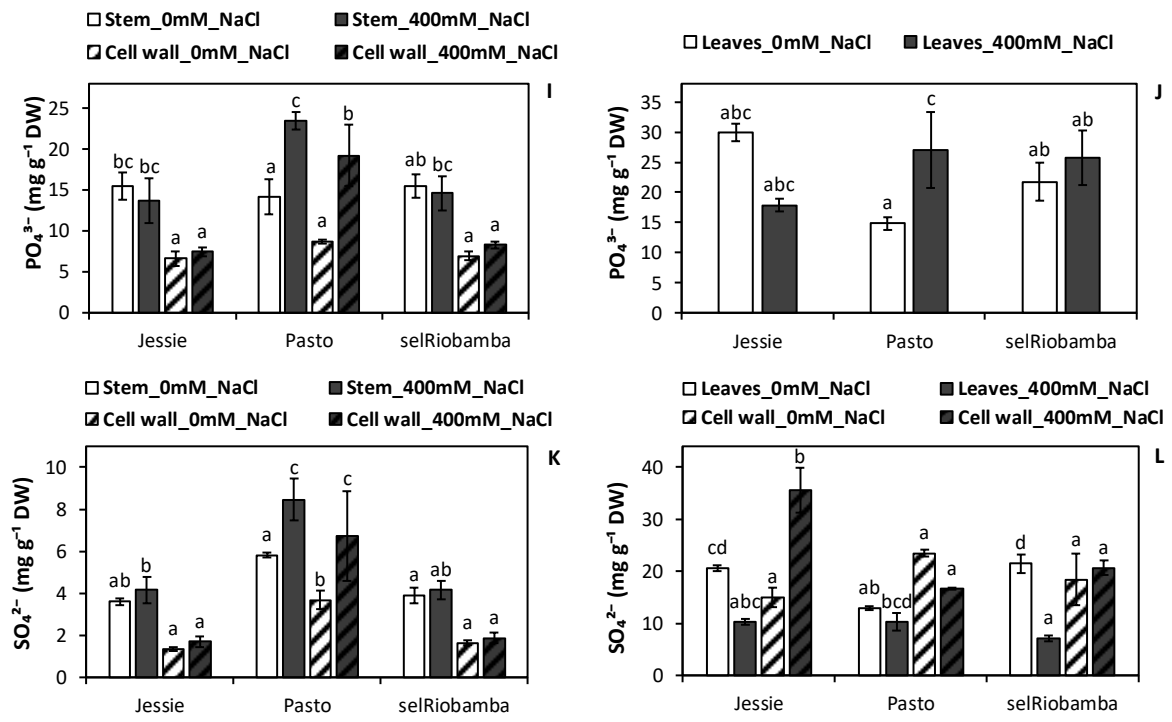

Figure 5. (continued) 


\section{Discussion}

Salt stress can affect cell wall properties in several ways. It affects the source-sink balance, which will result in a remodelling of the cell wall structure. In addition, induces structural changes in the cell wall to adapt to a new osmotic environment. Furthermore, the ions (in particular $\mathrm{Na}^{+}$) can interact with cell wall polymers (Byrt et al. 2018). By analysing the cell wall components in leaves and stems of salt-stressed plants, we intend to better understand the functional adaptations of the quinoa cell wall that contribute to its extraordinary resilience to adverse conditions.

The monosaccharide profiles of the cell walls show a significant salt-induced increase of galacturonic acid, arabinose, galactose and rhamnose, which is indicative of an increase in the pectin fraction. Pectins have an important role in plant cell growth by facilitating cell wall extensibility (Cosgrove 2015). In response to drought or salt stress, changes in the pectin content or composition can increase cell wall elasticity and so, contribute to the maintenance of cell turgor (Le Gall et al. 2015). Increases in at least one type of pectin in response to salt stress have been reported in maize (Uddin et al. 2013), tolerant varieties of wheat (Leucci et al. 2008) and soybean (An et al. 2014), coffee (de Lima et al. 2014), tobacco (Iraki et al. 1989). In our experiment, arabinose had the highest difference between control and salt treatment; it was increased by $160 \%$ in stem cell walls and $60 \%$ in leaf cell walls of all the examined varieties under salt stress. Arabinose is a building block for arabinans and arabinogalactans, major components of the pectin polymer $R G I$, is an important residue in RGII, and is also part of glucoarabinoxylan of the hemicellulose polymer, glycoproteins such as extensins, and arabinogalactan proteins (Zhao et al. 2019). Arabinans, galactans and highly branched arabinogalactans are main determinants of the hydration status of the cell wall matrix due to their high water binding capacity and ability to form gels (Klaassen and Trindade 2020). The observed increase of arabinose due to salt stress may be indicative of an increase of branching polymers within the pectin matrix. The importance of these branching polymers in the context of water deficit has been extensively explored in desiccationtolerant species (Jung et al. 2019; Moore et al. 2008b). These so-called resurrection plants are able to maintain cell turgor under severe drought stress by increasing cell wall flexibility with components described as "cell wall plasticizers", in particular arabinose-rich polymers (Moore et al. 2013). The model proposed for desiccation tolerant species states that arabinose-rich polymers are responsible for buffering/replacing the loss of water by their gelling capacity, and consequently preventing the formation of tight junctions between backbone chains (HG, cellulose 
microfibrils). These adjustments help to maintain the flexibility of plant cell walls during growth and to adapt to changes in the water status/turgor of the cell (Moore et al. 2008a). Our results suggest that severe salt stress induces an increase in arabinose-branching polymers in the cell walls of quinoa that might contribute to the hydration properties of the cell wall, the maintenance of cell turgor and the adaptation of this halophyte species to salinity.

Plants exposed to high salinity might suffer from the accumulation of $\mathrm{Na}^{+}$ ions in the apoplast, which can interact with negatively charged cell wall polymers. It has been established that $\mathrm{Na}^{+}$can displace pectin-bound $\mathrm{Ca}^{2+}$, disrupting pectin crosslinking (Byrt et al. 2018). Our data constitute experimental proof that the $\left[\mathrm{Ca}^{2+}\right]$ in the cell wall is substantially reduced under high salinity, while $\left[\mathrm{Na}^{+}\right]$increases. Pectin links are important for strengthening the cell wall, so this disruption will loosen the cell wall. The stiffness of the cell wall must be restored, possibly by the recovery of the levels of $\mathrm{Ca}^{2+}$ or the remodelling of other cell wall components (Rui and Dinneny 2020). It is known that arabinose-branching polymers interfere with $\mathrm{Ca}^{2+}$ induced pectic crosslinking (Cankar et al. 2014). Therefore, we propose that the increase of arabinose branching polymers may be also associated with the remodelling of the cell wall in salt tolerance species to compensate for the decreased levels of $\mathrm{Ca}^{2+}$ and crosslinking in pectins are.

The rise in the pectin-related monosaccharide fractions of the salt-stressed cell walls was accompanied by a significant decrease in both glucose and lignin content in cell walls of stems and, to a lesser extent, of leaves. It has been reported that salt stress affects cellulose biosynthesis and the underlying mechanism has been well described (Wang et al. 2015). Salt stress promotes the disassembly of microtubules, which impacts cellulose synthesis by plasma membrane-located cellulose synthase complexes (CSCs). In response to these salt stress-induced changes, the activity of companion cellulose synthase proteins (CC) that interact with CSCs and microtubules may be adjusted. This interaction contributes to restoring the assembly of microtubule and promotes the activity of CSCs, thus restoring the cellulose synthesis and helping the plant to cope with long-term salinity at a cellular level (Endler et al. 2015). Our results suggest that the cellulose content of quinoa cell walls decreases under salinity. Whether this is a direct effect of salt on the biosynthesis of cellulose or the result of adaptive remodelling of the cell wall to the metabolic status and physiology of the plants under salinity remains to be established. 
Increased lignification has been reported as a common response of several glycophytic species to salt stress (Le Gall et al. 2015). A higher deposition of lignin might increase mechanical strength and water impermeability of the cell wall (Cabane et al. 2012). Our results indicate that lignin content of salt-stressed quinoa is decreased, which indicate a different cell wall remodelling than other species. In addition, the lower content of lignin and cellulose in quinoa cell walls under salt stress suggests a decrease in the deposition of secondary cell wall. Secondary cell walls are observed in mature cells that have stopped growing (Doblin et al. 2010). Perhaps a lower content of secondary cell wall in quinoa tissues is related to a lowered and slower growth rate of the plants under salinity.

The sulphate content of the cell wall was significantly increased under high salinity. Due to the washing steps during the cell wall extraction procedure, it is likely that the measured sulphate was bound to specific polymers. Although sulphated polysaccharides are apparently absent in glycophytes, sulphated arabinogalactans play an important role in salt stress tolerance of green algae (Chlorophyta) and some reports suggest the presence of these components in some halophytes (Aquino et al. 2011; Corrêa-Ferreira et al. 2019). Different biochemical tools are needed to corroborate the presence of sulphated polysaccharides in quinoa cell wall or to understand where they are located in the cell wall. The role of sulphated polysaccharides in the response to salt stress and salt tolerance remains unknown. It has been suggested that these components might stabilize the negative charge of the cell wall in the presence of an excess of monovalent cations, thus favouring apoplastic transport and slowing down the movement of $\mathrm{Na}^{+}$towards the cells (Aquino et al. 2011).

The remodelling of the cell wall under adverse conditions appears to depend on the severity of the stress (Houston et al. 2016). We have examined the changes in the biochemical composition of the cell wall of quinoa exposed to severe salinity. As a facultative halophyte, quinoa is able to tolerate soil salinity levels that are considered severe for most crops without excessive yield penalties. At salinity levels that are close to that of seawater (the stress level applied in the experiment described here) quinoa switches to a halophytic strategy in which survival is favoured with a trade-off for growth and productivity (Jaramillo Roman et al. 2020). It remains to be tested whether the biochemical remodelling of the cell wall described in this study is part of the survival strategy of quinoa, or whether these modifications will also contribute to increased salt tolerance at lower salinity levels. A multidisciplinary approach including physiology, biochemistry, proteomics and genetics, as well as immunolabeling-based methodologies to target specific arabinans, AGPs and 
arabinoxylans, is needed to understand the complex functional cell wall adaptations to salt stress. It will also facilitate identification of genetic variation for these traits that may be used to further improve salt tolerance of the crop quinoa.

Currently we are investigating cell wall monoclonal antibodies using an inmunomicroscopy-based methodology to identify which cell wall polymer fractions (i.e. arabinans, AGPs, arabinoxylans) are adjusted under salinity. This information will further enhance our knowledge on how quinoa cell wall remodelling may contribute to the remarkable salt tolerance of this crop.

\section{Acknowledgments}

We acknowledge Dr. Jordi Petit for his technical guidance with the biochemical methods. We also acknowledge the expert assistance of Geurt Versteeg in the greenhouse. Viviana Jaramillo is financially supported by a scholarship from the Secretariat for Higher Education, Science, Technology and Innovation of the Republic of Ecuador (Contract Number 2015-AR2Q8878). 



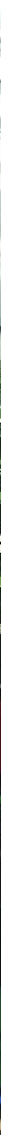

\section{Chapter 4 High-resolution analysis of growth and transpiration of quinoa under saline conditions}

Viviana Jaramillo Roman, Rick van de Zedde, Joseph Peller, Richard G.F. Visser, C. Gerard van der Linden, Eibertus N. van Loo 


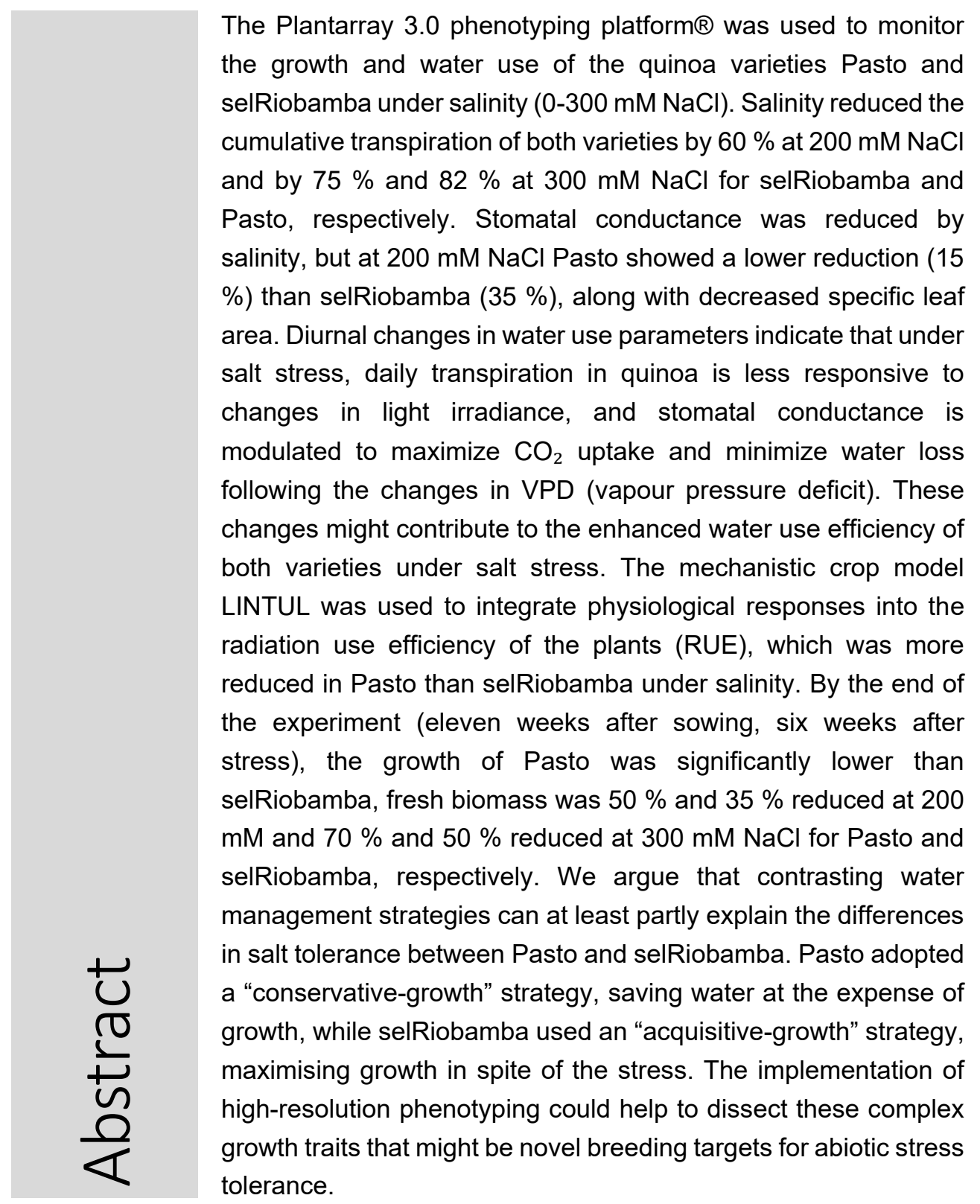




\section{Introduction}

Plant breeding for abiotic stress tolerance has proven to be complex (Gilliham et al. 2017). A major challenge is that stress tolerance is a systemic process that involves a number of synchronized, interconnected physiological processes and genes operating together. A second important complication is that these physiological processes are largely and continuously influenced by the environment. Thus, a proper screening of tolerance traits would ideally involve continuous monitoring of the plant responses to changes in the environment, which means that an accurate physiological phenotyping of well-defined traits is essential for the successful breeding for salt tolerance. Plant phenotyping has rapidly evolved in the past decades and has benefited enormously from developments in other disciplines such as remote sensing, robotics, computer vision and machine learning (Furbank and Tester 2011). Most state-of-the-art phenotyping facilities, particularly for stress-related traits, collect information using robotics and automated image acquisition and analysis: image-based phenotyping (Fahlgren et al. 2015). A complementary platform implements physiology-based gravimetric systems that enable the direct measurement of plant dynamic responses, also called functional phenotyping (Negin and Moshelion 2017). The data provided by gravimetric platforms, together with controlled measurements of environmental parameters, such as radiation, humidity, atmospheric vapour-pressure deficit (VPD) and temperature provide new insights into the complex genotype $\mathrm{x}$ environment interactions under specific treatments or abiotic stresses (Negin and Moshelion 2017).

Soil salinization is a major limiting factor for agriculture, causing significant pressure on the availability of arable land. Saline soils constitute more than $20 \%$ of the global irrigated land and affect agricultural production in more than 75 countries. Soil salinity causes severe yield and economic losses, especially to smallholder farmers worldwide, and is expected to expand as a result of climate change (Qadir et al. 2014). Plant growth is directly and indirectly affected by soil salinity. Growth takes place by the conversion of photosynthates into structural molecules. Energy from photosynthesis is also needed to maintain several physiological functions that rely on assimilation of carbon dioxide and glucose metabolism, known as maintenance respiration (De Vries 1975). When salt accumulates in the soil, the osmotic potential decreases, and the osmotic gradient between root medium and the roots leads to reduced water uptake with a subsequent reduction in cell expansion (Munns 2002). By adjusting internal osmotic potential by for instance the accumulation of inorganic and/or organic compounds, plants can restore water 
uptake, at least up to a certain degree. The salinity-induced water uptake limitation directly affects growth through decreased $\mathrm{CO}_{2}$ availability resulting from stomata closure and down-regulation of photosynthetic metabolism (Chaves et al. 2009). The indirect effects of salt stress on growth include possible damage to the photosynthetic machinery caused by the secondary oxidative stress prompted by salinity as well as an increased maintenance respiration caused by several costly salt stress response mechanisms (i.e. osmotic adjustment, ion transport) (Karlberg et al. 2006). Due to the increased maintenance respiration less assimilates will be available for plant growth with the same amount of transpired water, which would lead to a decreased water use efficiency (Munns et al. 2020a).

The assessment of plant growth and the relation with transpiration and transpiration efficiency would provide a mechanistic account of the salinity effects at the whole plant level. However, transpiration has always been a trait that is laborious and expensive to measure and this has limited its incorporation in salt tolerance studies. Therefore, we only have fragmented understanding of the consequences of salinity-induced changes in transpiration on growth and yield reduction (Harris et al. 2010). In the present study we use a functional phenotyping platform based on minilysimeters, the Plantarray 3.0 platform (Plant-Ditech, Rehobot, Israel). This platform allows simultaneous and high temporal resolution measurements of water uptake, transpiration and plant growth to expand our understanding of salt stress responses of plants, using the facultative halophyte Chenopodium quinoa as a model species.

Chenopodium quinoa is an herbaceous, annual crop that originated in the Andes and is well-adapted to harsh environments, such as nutrient-poor, droughtaffected, and saline soils. The high salt tolerance of quinoa has been widely recognized (Hinojosa et al. 2018; Jaramillo Roman et al. 2020). The overall goals of this study are: i) to explore the interacting effects of salt stress on water uptake, transpiration and growth of quinoa, ii) to identify salt tolerance strategies of two quinoa genotypes known to differ in their response to salinity and iii) to examine the potential of high-resolution functional phenotyping for identifying physiological markers for salt tolerance screening in breeding programs. 


\section{Materials and Methods}

\subsection{Plant materials and treatments}

The European non-bitter quinoa varieties Pasto and selRiobamba, a line selected from Riobamba (Riobamba has still some residual heterozygosity) were used in this experiment. These varieties were bred at Plant Breeding, Wageningen University \& Research (The Netherlands) and AbbottAgra (France) and in previous experiments they have shown contrasting responses to salt stress (Jaramillo Roman et al. 2020). The experiment was conducted between March and May 2019 at the Unifarm greenhouse facilities of Wageningen University \& Research, The Netherlands. Plants were sown in trays filled with potting soil and transplanted to 4 $L$ pots 16 days after sowing (DAS). The pots were filled with standard filtered sand (grain size $0.6-1.0 \mathrm{~mm}$ ) and each pot contained 4 plants. To prevent evaporation, small PVC balls were put on the surface of the pots, surrounding the plants. The greenhouse air humidity was set to a minimum of $80 \%$ and the photoperiod to $16 \mathrm{~h}$ light. When the incoming shortwave radiation was below $200 \mathrm{Wm}^{-2}$, additional lighting was supplied $\left(100 \mathrm{Wm}^{-2}\right)$. The plants were irrigated with half-concentrated Hoagland's nutrient solution. Salt stress treatment started 33 DAS with irrigation with 0.5 x Hoagland's solution plus $200 \mathrm{mM} \mathrm{NaCl}$. However, due to the sudden increase to $200 \mathrm{mM} \mathrm{NaCl}$ salinity, wilting was observed in the leaves of treated plants a few hours after irrigation. Therefore, the excess of salt was washed out and the salt treatment was built up in incremental steps of $100 \mathrm{mM} \mathrm{NaCl}$ per day until the desired salt concentration was reached. The final salt treatments of $200 \mathrm{mM}$ and $300 \mathrm{mM}$ $\mathrm{NaCl}$ were reached on day 36 after sowing, and the soil salt concentration in the drainage was monitored continuously with a conductivity meter (Profile Cond $315 \mathrm{i}$, Xylem Analytics, Germany) for the duration of the experiment. Four pots per variety were used in each treatment. Half of the plants (2 plants per pot) were harvested 47 days after sowing and the remaining plants were harvested 77 days after sowing. During the first destructive harvest, the above-ground biomass was collected and separated into stems, leaves and inflorescences. Leaves were split in young (upper one-third of the plant) and old leaves (lower two-thirds of the plant). Fresh weights of leaves, stems and inflorescences were recorded, and leaf area was measured using a leaf area meter (Li-3000 Area Meter, Li-Cor, Lincoln, NE, USA). During the second destructive harvest, roots were collected as well and weighed. Dry weights were determined after drying the samples in a forced-air oven at $60^{\circ} \mathrm{C}$ until they reached stable weight. The salt tolerance index (STI) was calculated as the ratio of 
above-ground dry biomass of salt-treated plants and the dry biomass of control $(0$ $\mathrm{mM} \mathrm{NaCl)}$ plants.

\subsection{Plantarray design and data collection}

The functional phenotyping platform Plantarray 3.0 platform (Plant-Ditech, Rehobot, Israel) was used to monitor plant growth through controlled tracking and measuring of irrigation and biomass increase throughout the growing period. The system uses highly sensitive load cells that are used as weighing lysimeters. Additional sensors were incorporated to the system in order to monitor other environmental factors. These were: HC2-S3-L meteo probe for relative humidity and temperature in the greenhouse (Rotronic, Crawley, UK), LI-COR 190 Quantum Sensor for photosynthetically active radiation measurements (Lincoln, NE, USA), and a soil moisture, electro-conductivity and temperature sensor (5T, Decagon devices, Pullman, WA, USA) incorporated in every pot. Each load unit (containing one pot) was connected to an individual control unit (CR1000 data logger) (Campbell Scientific, Logan, UT, USA) (Figure 1A). The system recorded the weight of the pots plus the environmental information registered by the sensors every 3 min. The data collection could be viewed in real-time through the online web-based software SPAC-analytics (Plant Ditech, Rehobot, Israel). The physiological traits could not directly be extracted from the protocols implemented by the SPAC analytics software, because at the beginning of the experiments the seedlings were very small and despite the use of the PCV balls the effect of evaporation was considerable, and therefore the weight of the pots could not be equilibrated. Several pots containing only substrate were placed next to the system and weighed manually on a daily basis to estimate evaporation from the pots.

Additional pots were grown next to the system for the two varieties (8 pots with 4 plants each) and harvested throughout the experiment for growth rate calculations. Several destructive harvests were performed on this material: 1) when seedlings were transplanted from trays to pots (16 days after sowing (DAS)), 2) when pots were incorporated to the system (26 DAS), 3) when the salt treatment started (36 DAS). The harvested material was used to measure leaf weight ratio (LWR) (g $\left.\mathrm{g}^{-1}\right)$, and specific leaf area (SLA) $\left(\mathrm{m}^{2} \mathrm{~kg}^{-1}\right)$. SLA was calculated as the amount of leaf area per unit of leaf dry weight, LWR as the leaf fraction of the total dry plant biomass. RGR was calculated as the natural logarithm of the relative increase in plant biomass over the mentioned period of time: $R G R=\ln \left(W_{2} / W_{1}\right) /\left(t_{2}-t_{1}\right)$ (Lambers and Poorter 1992). Net assimilation rate (NAR, $\mathrm{g} \mathrm{m}^{-2}$ day $^{-1}$ ) was derived using the linear relation $R G R=L W R \times S L A \times N A R$. The RGR components calculated per 
period for the plants in the extra pots were used to estimate the RGRs of the plants in the system. To do so, it was assumed that the plants only grew during the light hours, and that RGR was strongly correlated with PAR during the day. This allowed us to derive RGRs for the plants in the system from the RGRs measured on the extra pots. Using the initial weight of the seedlings at the start of the experiment and assuming exponential growth of the plants, the derived RGRs were used to calculate fresh weights $(F W)$ of the plants in the system with a 3-minute resolution. The measured fresh weights of the first three destructive harvest of the extra pots (16, 26 and 36 DAS) and the measured fresh weights on the plants on the system at 47 and 77 DAS were used as reference values to validate the calculation of FW values throughout the experiment.

The interpolated RGRs and FWs were used to estimate the other components of the RGR analysis (LWR and SLA) at each individual timepoint, and to obtain a reference value for the leaf area. Transpiration rate per time point equates rate of water loss from the pots, corrected for evaporation. This was calculated using the weight values of pots plus plants provided by the system and subtracting the interpolated FW and the weight of static components added to the lead cells. Correction for evaporation was done based on the evaporation rates of the extra pots without plants. A running average of $180 \mathrm{~min}$ was used in the calculations to account for possible missing values in the weights provided by the lysimeters. Stomatal conductance ( $g s$ ) was calculated as transpiration rate/ leaf area/ VPD \%. A running average of 120 minutes of data that was recorded every 3 minutes was used to correct for possible errors or outlier values in the system or VPD measurements. To validate the calculated $g s$, a portable leaf porometer (Decagon Devices Inc., WA, Australia) was used to measure gs on the abaxial side of the second fully developed non-shadowed leaf between 12:00-13:00 hours at 58 DAS. Finally, whole plant water use efficiency (WUE) was calculated as g FW/ g water transpired for an interval of $3 \mathrm{~min}$ in a $2 \mathrm{~h}$ running average using the interpolated fresh weights and transpiration rates.

\subsection{Integrating phenotyping data to a crop production model}

The mechanistic crop growth model LINTUL (Light interception and utilisation) was used as a framework to integrate several physiological components to plant growth (Spitters and Schapendonk 1990). LINTUL is based on the linear relationship between produced biomass and the amount of radiation intercepted by the crop. The crop growth rate is calculated as: $\mathrm{d} W_{t} / \mathrm{dt}=f_{t} \times P A R_{t} \times R U E$, where $\mathrm{d} W_{t} / \mathrm{dt}$ is the instantaneous growth rate at day $\mathrm{t}\left(\mathrm{g} \mathrm{DM} \mathrm{m} \mathrm{m}^{-2} \mathrm{~d}^{-1}\right), P A R_{t}$ the 
incoming amount of photosynthetically active radiation ( $M J \mathrm{~m}^{-2} \mathrm{~d}^{-1}$, 'light' wave bands $400-700 \mathrm{~nm}$ ), $f_{t}$ the fraction of PAR intercepted by the foliage, and RUE the average light utilization efficiency or radiation use efficiency (g DM MJ-1 PAR). The fraction of light intercepted during exponential growth can be calculated as $1^{\text {-exp(-kLAl) }}$ on the basis of simulated LAI, where LAl is the leaf area index $\left(\mathrm{m}^{2}\right.$ leaf surface $\left(\mathrm{W}_{\mathrm{t}} \mathrm{X}\right.$ LWR $\times$ SLA) $\mathrm{m}^{-2}$ ground surface) and $k$ is the extinction coefficient (Spitters and Schapendonk 1990). Based on several studies that applied the LINTUL crop growth model (Sinclair and Muchow 1999), the radiation extinction coefficient (k) was assumed to be 0.8 for this experiment, and the area of the pot that intercepted light (based on a pot size of $40 \times 60 \mathrm{~cm}$ ) to be $1 \mathrm{~m}^{2}$. Following an Expo linear model, RUE can be related to the RGR through the following relations: $1 / W_{t} \times d W_{t} / d t=$ $R G R=L W R \times S L A \times N A R$, thus $N A R=P A R_{t} \times R U E($ Van Loo 1992).

\subsection{Rapid light curve}

Chlorophyll fluorescence measurements were performed at 76 DAS using the stand device Robin PSI PlantScreen TM system (Photon System Instruments, Brno, Czechia) for kinetic chlorophyll fluorescence analysis. The device is equipped with a chlorophyll fluorescence imaging unit FluorCam FC-800 mF Pulse Amplitude modulated (PAM). Three detached young leaves per plant were introduced in the device to perform the analysis. Rapid light curves were measured following 20 seconds acclimation at six different actinic light intensities (10-20-40-60-80-100 \% of a maximum actinic light of $\left.1692 \mu \mathrm{mol} \mathrm{m} \mathrm{m}^{-2} \mathrm{~s}^{-1}\right)$ for a duration of $10 \mathrm{~s}$. The calculated parameter was the PSII effective quantum yield ( $\left.\varphi_{P S I I}\right)$ defined as $\left(F^{\prime} \mathrm{m}-F^{\prime}\right) / F^{\prime} \mathrm{m}$ where $F^{\prime}$ is the fluorescence emission from a light-adapted leaf and $F^{\prime} m$ is the maximal efficiency from a light-adapted leaf. Relative electron transport rate (rETR) is an approximation of the rate of electrons pumped through the photosynthetic chain, and was estimated as: $\mathrm{rETR}=\varphi_{P S / I} \times \mathrm{PAR} \times 0.85 \times 0.5$ where 0.85 is the value for absorption coefficient of the leaves and 0.5 the fraction of excitation energy distributed to PSII (Tschiersch et al. 2017).

\subsection{Thermal imaging of quinoa leaves}

A thermal camera (FLIR A655sc, FLIR Systems, INC., Wilsonville, UE) was mounted above the plants. This camera has a $640 \times 480$ pixels resolution, and a temperature range of $-40{ }^{\circ} \mathrm{C}-150{ }^{\circ} \mathrm{C}$, with a spectral range of $7.5-14 \mu \mathrm{m}$. The camera was allowed to automatically perform a NUC calibration throughout the period of imaging (between day 58 and 66 after sowing). One picture frame was recorder every ten minutes and the leaf temperatures were measured in a circular region in the centre of a young leaf of each plant as depicted in Figure 5A. 


\subsection{Ion content measurements}

The ion content of young and old leaves, stems and roots was measured using lon Chromatography (IC) system 850 Professional (Metrohm Switzerland). For this purpose, oven-dried tissues were ground to fine powder using a hammer mill with $1 \mathrm{~mm}$ sieve. Twenty-five $\mathrm{mg}$ per sample was ashed in a furnace at $550{ }^{\circ} \mathrm{C}$ for 5 h. Ten $\mathrm{ml}$ of Milli-Q ${ }^{\circledR}$ water was added to the ashes and these were shaken for 15 $\min$ at $5000 \mathrm{rpm}$ at $100{ }^{\circ} \mathrm{C}$. Prior to injection onto the IC system, samples were diluted 400 times with Milli- $Q^{\circledR}$. Ion contents were calculated as the amount of ions per unit of dry weight ( $\mathrm{mg}$ ion $\mathrm{g}^{-1}$ dry mass) and the ion concentrations were estimated based on the water content of the tissue. The ratio $\mathrm{K}^{+} / \mathrm{Na}^{+}$was calculated based on $\mathrm{mg} \mathrm{K}^{+} / \mathrm{mg} \mathrm{Na}^{+}$content.

\subsection{Statistical analysis}

General analyses of variance (ANOVA) were performed to determine the significance of genotypic differences, salt treatment differences and their interactions $(p<0.05)$. The analyses were performed following a standard procedure for a linear mixed model, for which genotype and salt treatment were considered fixed effects and blocks random effects. The above-mentioned model was: $y_{i j k}=\mu+b_{k}+\alpha_{i}+$ $d_{i k}+\beta_{j}+\alpha \beta_{i j}+e_{i j k}$, were $y_{i j k}$ is the response variable, $\mu$ is the grand mean, $\alpha_{i}$ is the salt treatment effect, $\beta_{j}$ is the genotype effect, $\alpha \beta_{i j}$ is the genotype-by-salt interaction effect, $b_{k}$ and $d_{i k}$ are the block effects and $e_{i j k}$ is the residual error. Multiple comparison analyses were performed using Fisher's protected least significant difference (LSD) test on genotype means. All statistical analyses were performed using the software Genstat $19^{\text {th }}$ Edition (VSN International Hemel Hempstead, UK).

\section{Results}

\subsection{General salt stress response of the plants}

The effect of salt stress on the biomass and ion distribution in plant tissues of Pasto and selRiobamba was similar to previous evaluations (Jaramillo Roman et al. 2020). Two destructive harvests were carried out during this experiment. The first one at 47 DAS (11 days after the start of the stress) and the second one at 77 DAS (41 days after the start of the stress). After 11 days of salt stress, biomass was already significantly reduced and the average salt tolerance based on dry weight was $80 \%$ for the $200 \mathrm{mM} \mathrm{NaCl}$ treatment and $50 \%$ for the $300 \mathrm{mM} \mathrm{NaCl}$ treatment. At the second destructive harvest (6 weeks of salt stress), the impact of salinity on 
biomass was greater, with an average salt tolerance of $56 \%$ at $200 \mathrm{mM} \mathrm{NaCl}$ and $34 \%$ at $300 \mathrm{mM} \mathrm{NaCl}$ (Figure 1B). Both varieties were smaller but remained green and did not lose leaves despite the high salt treatments, but selRiobamba was significantly more salt tolerant than Pasto. The $\mathrm{Na}^{+}, \mathrm{K}^{+}$and $\mathrm{Cl}^{-}$concentrations were measured in roots, stems, old and young leaves at 77 DAS. The concentration of $\mathrm{Na}^{+}$in selRiobamba showed an increasing gradient from roots to stem to leaves, and the concentration was slightly lower in young leaves compared to old leaves. Pasto on the other hand, showed lower $\left[\mathrm{Na}^{+}\right]$in leaves compared to roots and stems. The $\left[\mathrm{Na}^{+}\right]$of Pasto in young leaves at the $300 \mathrm{mM} \mathrm{NaCl}$ treatment was $156 \mathrm{mM}$, compared to $531 \mathrm{mM}$ in selRiobamba (Supplementary Figure 1A). A similar trend was observed for the $\left[\mathrm{Cl}^{-}\right]$in different tissues. The highest concentration of $\mathrm{Cl}^{-}$for selRiobamba was measured in leaves, while Pasto showed lower levels of $\mathrm{Cl}^{-}$in young leaves compared to stems and roots (Supplementary Figure 1B). Salinity significantly decreased the $\left[\mathrm{K}^{+}\right]$of selRiobamba in all tissues. For Pasto, $\left[\mathrm{K}^{+}\right]$was not significantly affected by the $200 \mathrm{mM} \mathrm{NaCl}$ treatment and was significantly increased by an average of $20 \%$ in stems and young leaves at $300 \mathrm{mM} \mathrm{NaCl}$ (Supplementary Figure $1 \mathrm{C}$ ). The $\mathrm{K}^{+} / \mathrm{Na}^{+}$was higher in Pasto for all tissues and treatments. 


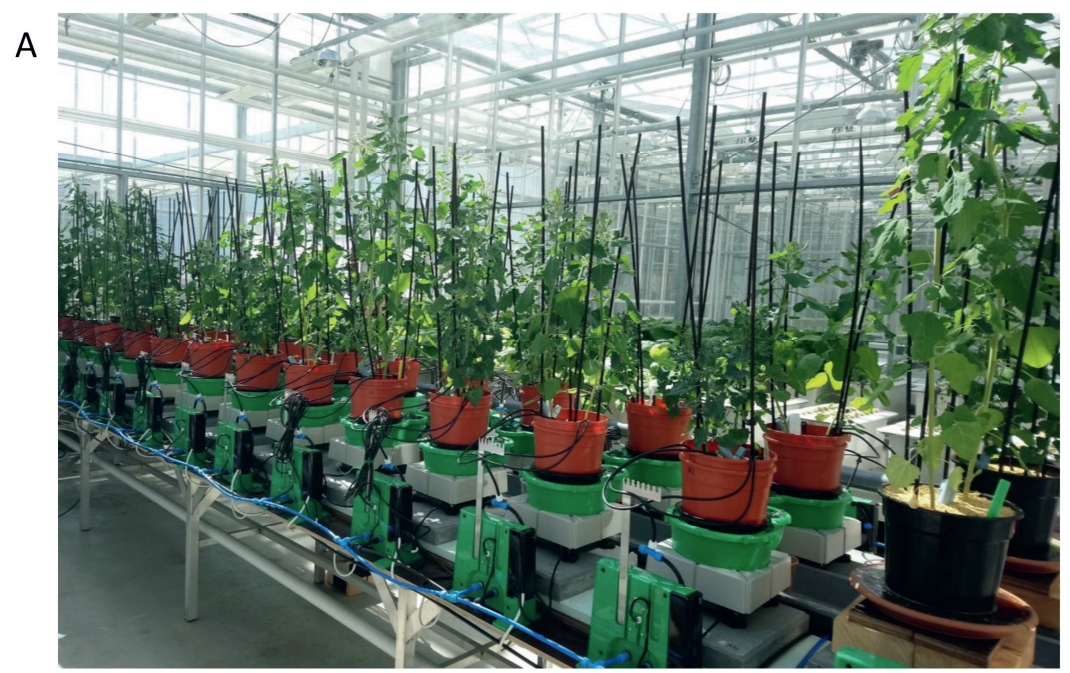

B
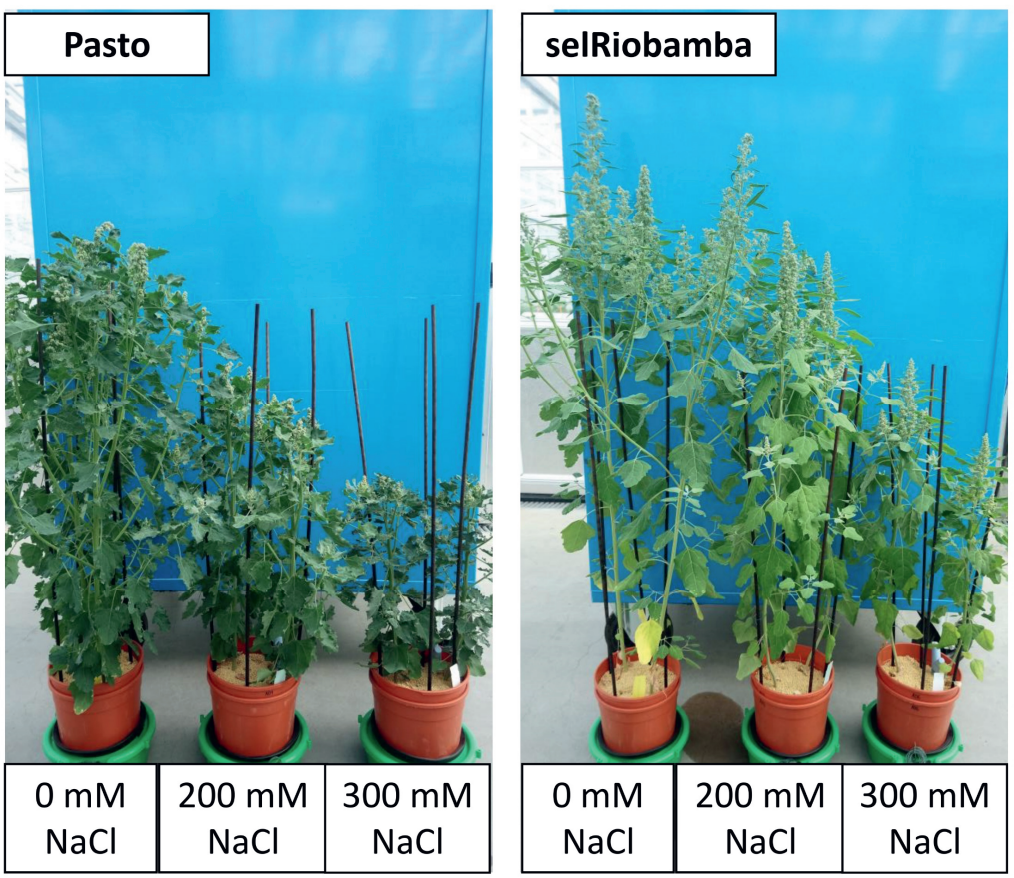

Figure 1. A) Plant Array 3.0 platform used in this study. Each pot is positioned in a sensitive load cell connected to a control unit. B) Pasto and selRiobamba at 77 DAS (6 weeks after the start of the salt treatment). 


\subsection{Monitoring plant growth and transpiration throughout the season}

The Plantarray phenotyping platform used in this study allowed us to monitor transpiration and biomass gain of plants continuously throughout the growing period (77 days). The cumulative water transpired by the plants is depicted in Figure 2A. Under control conditions, transpiration of Pasto and SelRiobamba was similar, in spite of their morphological differences (Pasto is a shorter variety and has higher leaf area per plant than selRiobamba) (Figure 2B). The salt treatment significantly affected the transpiration of plants. At $200 \mathrm{mM} \mathrm{NaCl}$, transpiration was reduced by on average $60 \%$. The more severe treatment of $300 \mathrm{mM} \mathrm{NaCl}$ had a stronger effect on transpiration and also accentuated the differences between varieties.
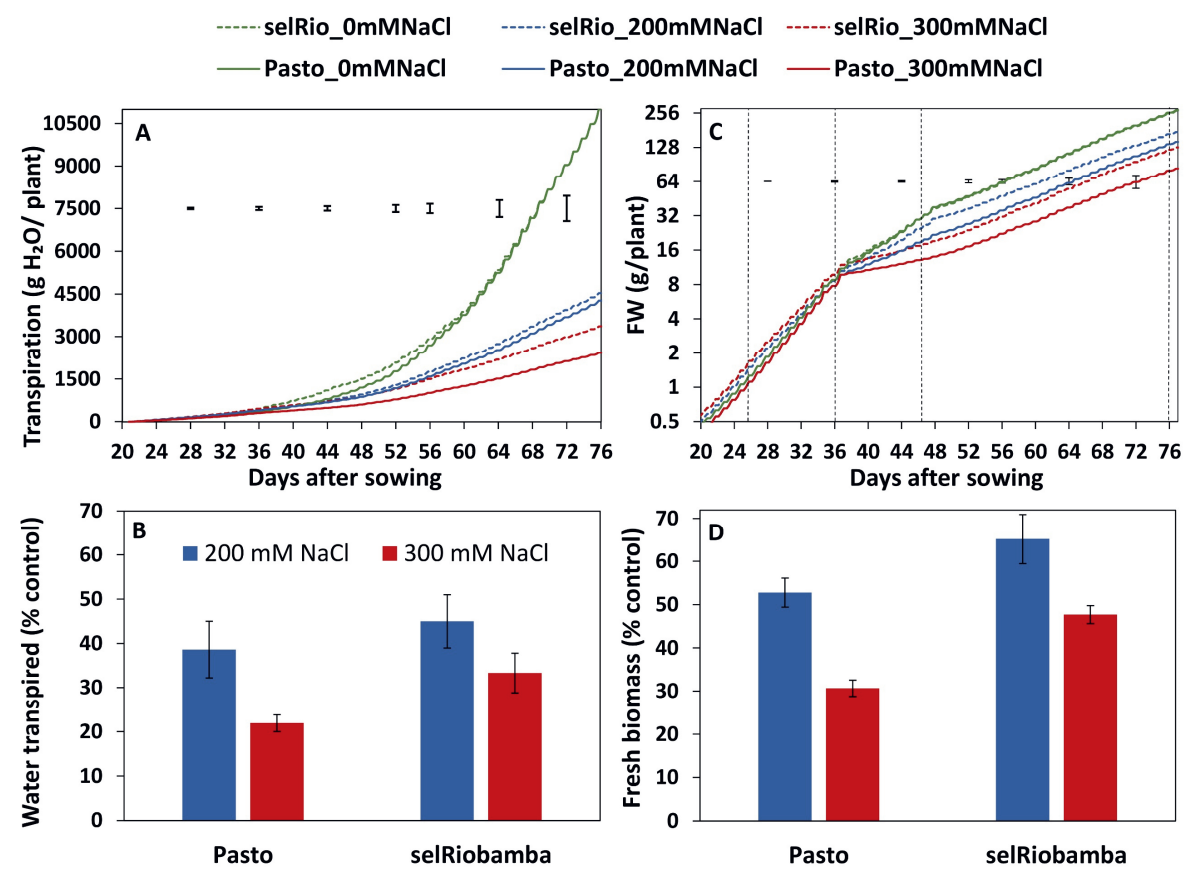

Figure 2. Dynamics of transpiration and growth throughout the season. A) Cumulative plant transpiration in two varieties (Pasto and selRiobamba) and three salt concentrations (control $(0 \mathrm{mM} \mathrm{NaCl}), 200 \mathrm{mM}$ $\mathrm{NaCl}$ and $300 \mathrm{mM} \mathrm{NaCl}$ ). B) Total water transpired by the plants at 77 DAS under salt stress as a percentage of the control. C) Fresh biomass per plant. Fresh weights were interpolated based on RGRs estimated from destructive harvest from extra pots (days 11, 21 and 36 after sowing) or plants growing in the system (47 and 77 DAS). The dotted black lines in the graph indicate the dates of the harvest in which the interpolated weights were validated with the biomass data from the harvests (16, 26, 36, 47, 77 DAS). D) Fresh biomass of the plants at 77 DAS under salt stress as a percentage of the controls. Means of 4 plants. Error bars indicate SE of individual means. 
By the end of the experiment, the average cumulative transpiration per plant was $11 \mathrm{~L}$ in control conditions, while at $300 \mathrm{mM} \mathrm{NaCl}$, transpiration was $66 \%$ lower for selRiobamba and $88 \%$ lower for Pasto (Figure 2B). The progressive accumulation of biomass was also monitored throughout the experiment (Figure $2 \mathrm{C}$ ). Salinity had a significant effect on the fresh weight of plants already after four days $(p<0.001)$. Throughout the season, growth rates and biomass accumulation of both varieties were not significantly different under control conditions and were reduced by salinity. Biomass was more reduced in Pasto than selRiobamba. By the end of the experiment (after six weeks of salt treatment), the fresh biomass of selRiobamba was $35 \%$ decreased under $200 \mathrm{mM} \mathrm{NaCl}$ and $50 \%$ decreased under $300 \mathrm{mM} \mathrm{NaCl}$, while Pasto biomass was $50 \%$ and $70 \%$ decreased under 200 and $300 \mathrm{mM} \mathrm{NaCl}$, respectively (Figure 2D).

\subsection{Variation in water use responses to salinity throughout the season}

Daily transpiration rate was calculated considering only the hours of light (Figure 3A). Salt-induced differences in the amount of water transpired were detected from the first day of salt treatment. Throughout the season, the transpiration rates were similar for the varieties under control conditions and under the lower salt treatment of $200 \mathrm{mM} \mathrm{NaCl}$. However, under $300 \mathrm{mM} \mathrm{NaCl}$, transpiration was clearly higher for selRiobamba. The differences in transpiration between salt treatments and varieties were significant. By the end of the experiment, the transpiration rate was reduced by $75 \%$ for selRiobamba under $300 \mathrm{mM} \mathrm{NaCl}$ and $82 \%$ for Pasto.

Stomatal conductance ( $g s$ ) was calculated using transpiration rates and interpolated leaf area data as described in Materials and Methods. Salt had a significant effect on stomatal conductance already three days after the start of the salt treatment (Figure 3B). Under $200 \mathrm{mM} \mathrm{NaCl}$, the gs for selRiobamba was $35 \%$ lower, while the gs for Pasto was $15 \%$ lower than control. Under $300 \mathrm{mM} \mathrm{NaCl}$, the gs for both varieties was reduced by $35 \%$.

Water use efficiency (WUE) at whole-plant level was calculated using Plantarray data as the ratio of cumulative biomass to cumulative water transpired. WUE was strongly influenced by the salt treatment throughout the growing period (Figure 3C). Shortly after the start of the salt treatment, WUE was lower at $300 \mathrm{mM}$ compared to control and the $200 \mathrm{mM} \mathrm{NaCl}$. However, a few days after the application of salt, WUE of the stressed plants exceeded the one of plants growing under control conditions. By the end of the experiment, WUE of both varieties at $200 \mathrm{mM} \mathrm{NaCl}$ was $56 \%$ higher than control. At $300 \mathrm{mM} \mathrm{NaCl}$, Pasto WUE was increased by $60 \%$ and selRiobamba WUE by $75 \%$ compared to the controls. 

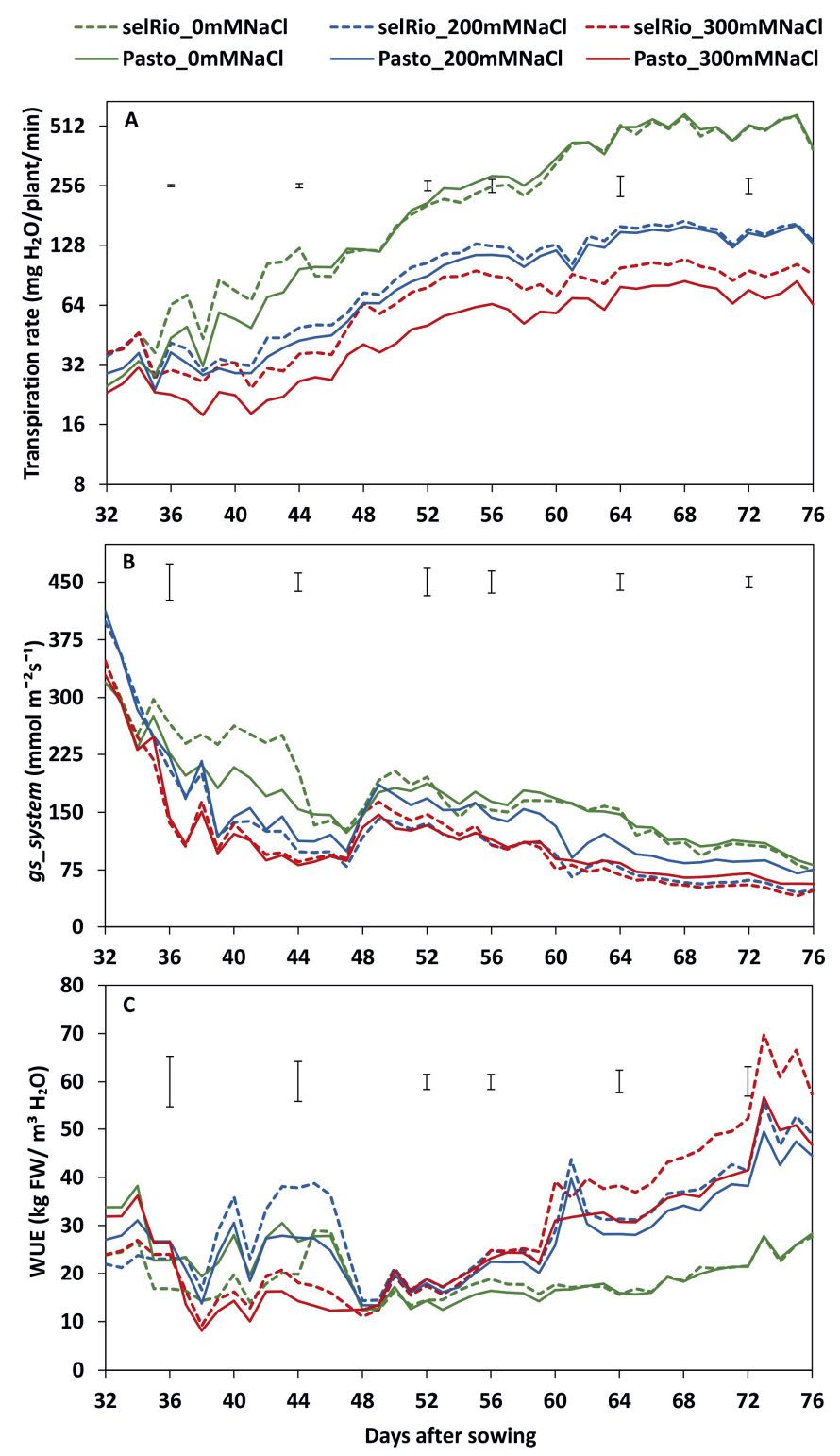

Figure 3. Water use parameters derived from Plantarray 3.0 data. A) Average transpiration rate per day considering the hours of light received by the plants in the greenhouse. B) Average stomatal conductance (gs_system) per day considering the hours of light received by the plants in the greenhouse. C) Average whole-plant agronomic water use efficiency (WUE) per day considering the hours of light received by the plants in the greenhouse. Means of 4 plants. Error bars indicate SE of individual means. 


\subsection{Plantarray derived versus porometer stomatal conductance}

The stomatal conductance derived from the Plantarray System data ( $\left.g s_{s y s t e m}\right)$ was validated by comparing with the stomatal conductance measured with a steady state porometer ( $g s_{\text {porometer }}$ ) at 58 DAS (21 days after start of salt stress). Similar to $g s_{s y s t e m}$, salt-treated plants had significantly lower leaf $g s_{\text {porometer }}$ than control plants and no significant differences were found between varieties (Figure 4A-B). A strong positive correlation of 0.95 was found between the gsporometer and the gssystem (Figure $4 \mathrm{C}$ ), indicating that the stomatal conductance calculations using the Plantarray data are valid and that the derived stomatal conductance is a reliable representation of stomatal behaviour.

$\square 0 \mathrm{mM} \mathrm{NaCl} \square 200 \mathrm{mM} \mathrm{NaCl} \square 300 \mathrm{mM} \mathrm{NaCl}$
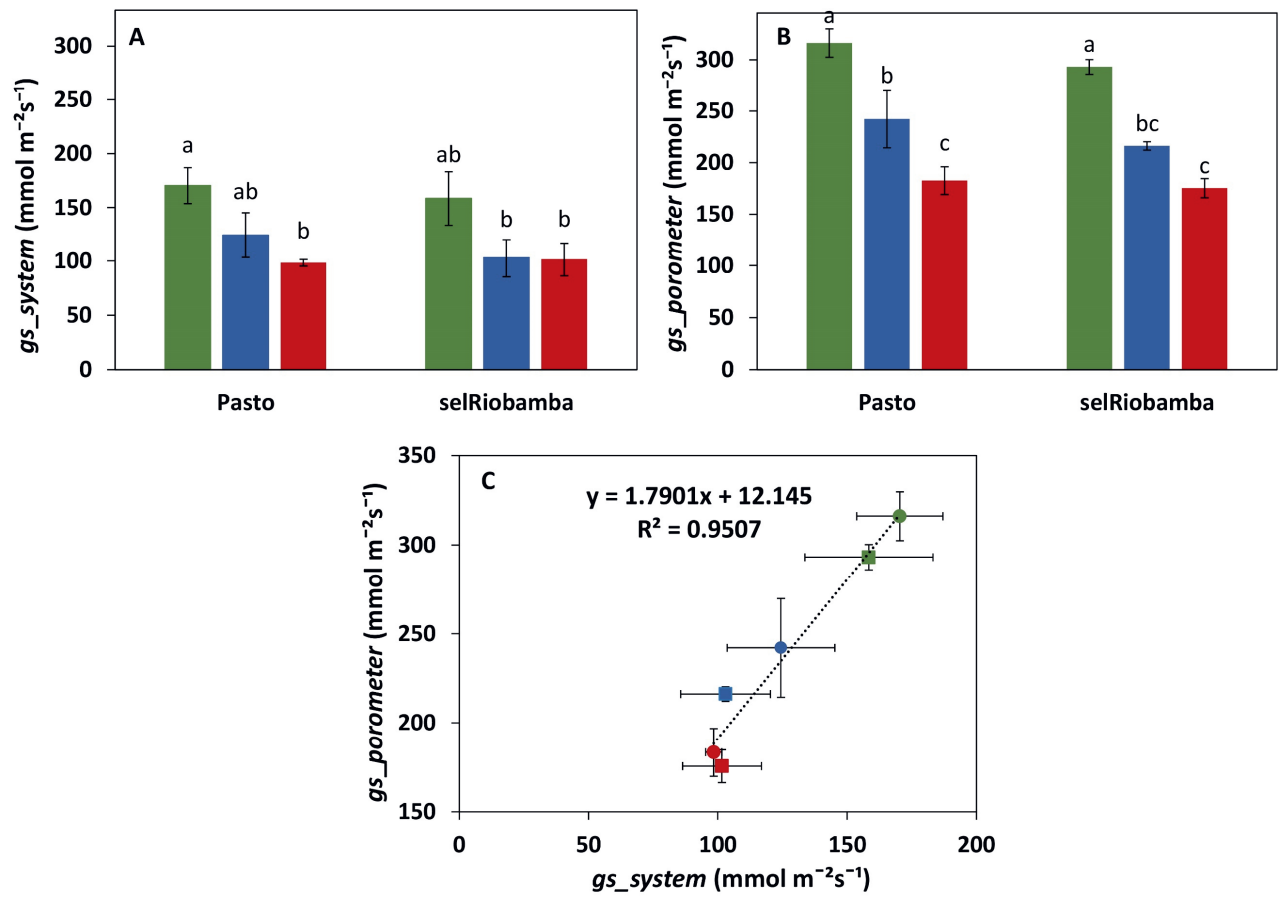

Figure 4. Comparison of the derived versus porometer stomatal conductance (gs). A) gs_system derived from Plantarray data as the average gs between 12:30 to 13:15 at 58 DAS. B) gs_porometer measured with a porometer from 12:30 to $13: 15$ at 58 DAS. C) Correlation between the derived from Plantarray versus porometer gs. Means of 4 plants. Error bars indicate SE of individual means. Statistically significant differences $(p \leq 0.05)$ between any variety and salt treatment combination are shown with different letters. 


\subsection{Thermal imaging as a surrogate estimation of stomatal conductance}

Infrared thermography phenotyping was used as an additional tool to monitor plant stomatal responses to salt stress from 58-66 DAS (39-45 days after the start of the stress) (Figure 5). Leaf temperatures were higher in the leaves of stressed plants compared to the controls (Figure 5A, B and C). On average, the difference between control and stressed plants was $2^{\circ} \mathrm{C}$, and the difference between the 200 and $300 \mathrm{mM} \mathrm{NaCl}$ treatments was about $1{ }^{\circ} \mathrm{C}$ (Figure 2C). The daily leaf canopy temperature and the daily gs calculated from Plantarray parameters were highly correlated $\left(R^{2}=0.9277\right)$ (Figure $5 D$ ).
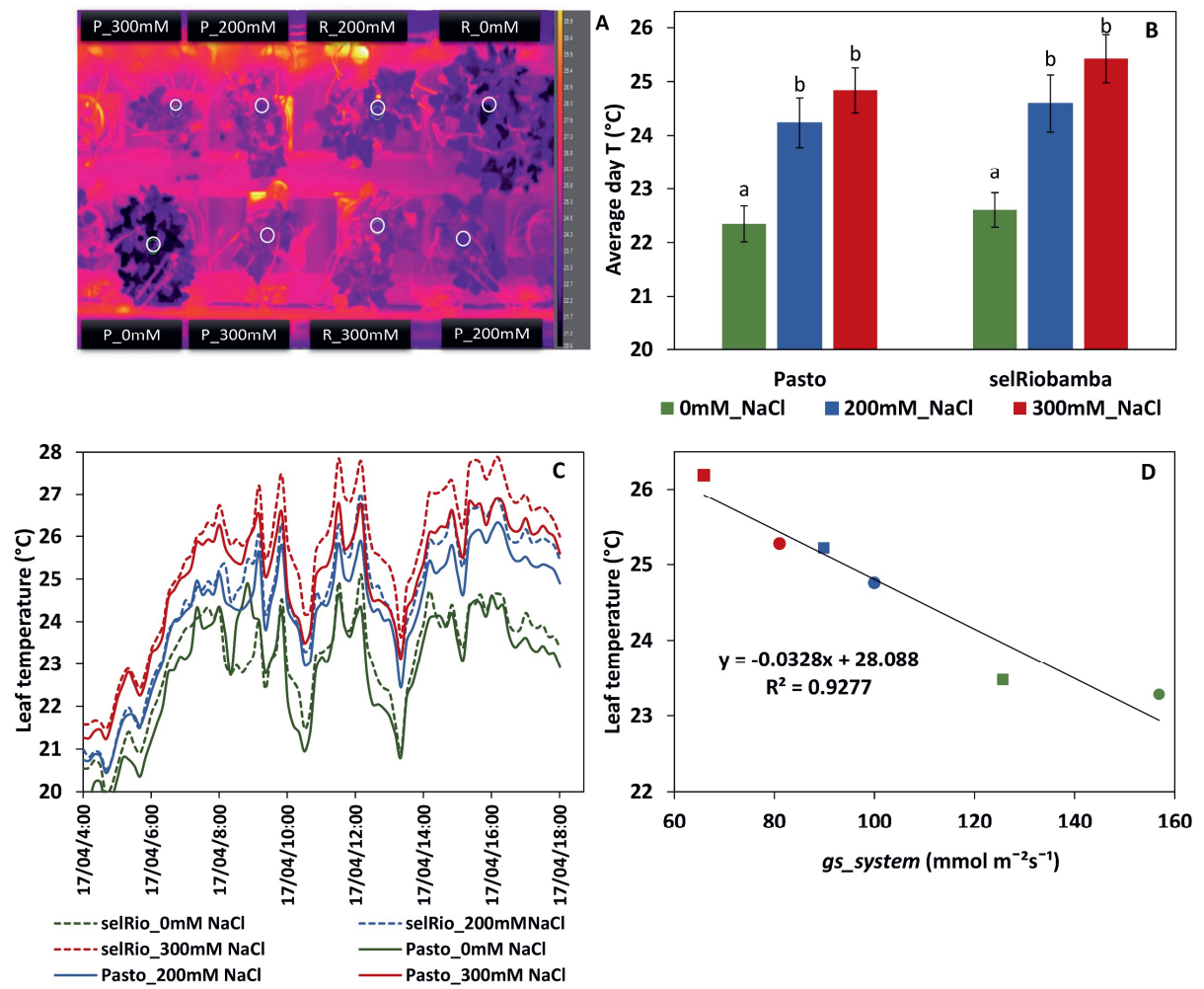

Figure 5. Leaf temperatures from thermal imaging for quinoa. A) Thermal image obtained by a FLIR A655SC Thermal Camera. White circles indicate the regions used to determine the mean leaf temperature per plant. B) Average leaf temperature during the day (07:00 AM-18:00 PM). Means of 7 consecutive days (59-65 DAS). Error bars indicate SE of individual means in the same plant. Statistically significant differences $(p \leq 0.05)$ between any variety and salt treatment combination are shown with different letters. C) Fluctuation of leaf temperature during a day (63 days after sowing, 42 days after start of the stress).

D) Correlation between leaf temperatures and gs_system derived from Plantarray data. 


\subsection{Variation in water use responses to saline conditions throughout the day}

The physiological traits measured in this study (transpiration rate, gs, WUE, leaf temperature) are influenced by environmental factors such as light intensity and VPD that vary between days and with the diurnal cycle. Figure 6 depicts the diurnal patterns of these parameters under control and saline conditions for two consecutive days (62-63 DAS, 41-42 days after the start of the stress). Light intensity and VPD showed considerable variation through the day and between days (Figure 6 A-B). The first day had higher irradiance levels than the second day. Maximum PAR on the first day was $830 \mu \mathrm{mol} \mathrm{m} \mathrm{m}^{-2} \mathrm{~s}^{-1}$ and occurred between 12:15- 13:15 hrs. On the second day, the distribution of light was more homogenous during the day, and the maximum PAR recorded was $380 \mu \mathrm{mol} \mathrm{m} \mathrm{m}^{-2} \mathrm{~s}^{-1}$. VPD patterns were similar to PAR patterns, with a maximum VPD of $3.5 \mathrm{kPa}$ measured during the first day and of 2.2 $\mathrm{kPa}$ during the second day. The transpiration rate during the same two days showed clear differences between treatments, varieties, and days (Figure 6C). Transpiration under control conditions followed the pattern of PAR. On the first (bright) day, the transpiration rate at the highest PAR level of the day was $740 \mathrm{mg} \mathrm{H}_{2} \mathrm{O} /$ plant/min for both varieties, compared to a maximum rate of $580 \mathrm{mg} \mathrm{H}_{2} \mathrm{O} /$ plant/min measured on the second more cloudy day. Under salt stress, transpiration rate was stable during the day and not significantly different between days, indicating that under saline conditions, transpiration is less responsive to changes in PAR. Stomatal conductance showed an early morning peak that declined as VPD increased and reached a plateau during the late morning and midday hours (Figure 6D). The morning gs showed a peak earlier under salt conditions than under control conditions, which may be a strategy to maximise $\mathrm{CO}_{2}$ absorption despite the lower transpiration rate. WUE for plants under control conditions was very stable throughout the day on both days, and similar for both varieties $\left(20 \mathrm{~kg} \mathrm{FW} / \mathrm{m}^{3} \mathrm{H}_{2} \mathrm{O}\right.$ ) (Figure 6E). WUE was significantly higher in plants under stress conditions. At 200 $\mathrm{mM} \mathrm{NaCl}$, WUE was similar for both varieties. During the first day, a max WUE of 65 $\mathrm{kg} \mathrm{FW/} \mathrm{m}^{3} \mathrm{H}_{2} \mathrm{O}$ was estimated around 11:00 AM (about one hour before the light irradiance and VPD max peaks recorded on the same day). During the second day with lower levels of irradiance, WUE values were also lower; the max WUE at 200 $\mathrm{mM} \mathrm{NaCl}$ was $42 \mathrm{~kg} \mathrm{FW/} \mathrm{m}^{3} \mathrm{H}_{2} \mathrm{O}$. The varietal differences were even more pronounced under $300 \mathrm{mM} \mathrm{NaCl}$ treatment, and the relative differences between the varieties were also higher on bright days. At peak irradiation on day one, WUE of selRiobamba was about $79 \mathrm{~kg} \mathrm{FW} / \mathrm{m}^{3} \mathrm{H}_{2} \mathrm{O}$, while the WUE of Pasto at the same time was $15 \%$ lower. In line with gs, leaf temperatures of stressed plants were 
significantly higher than control plants during the whole day (Figure 6F). Differences in temperature were clear from the start of the light period. The highest differences in temperature were observed during the afternoon (13:00-18:00) when the average leaf temperature of control plants was $21^{\circ} \mathrm{C}$ and of stressed plants as high as $25^{\circ} \mathrm{C}$. No significant temperature difference was observed between the 200 and $300 \mathrm{mM}$ $\mathrm{NaCl}$ treatment. Leaf temperatures were different between a bright and cloudy day, especially for stressed plants. The maximum temperature registered for leaves of plants growing at $300 \mathrm{mM}$ was $31^{\circ} \mathrm{C}$ at $13: 00 \mathrm{PM}$, while the temperature of control plants at the same time was $24^{\circ} \mathrm{C}$. 

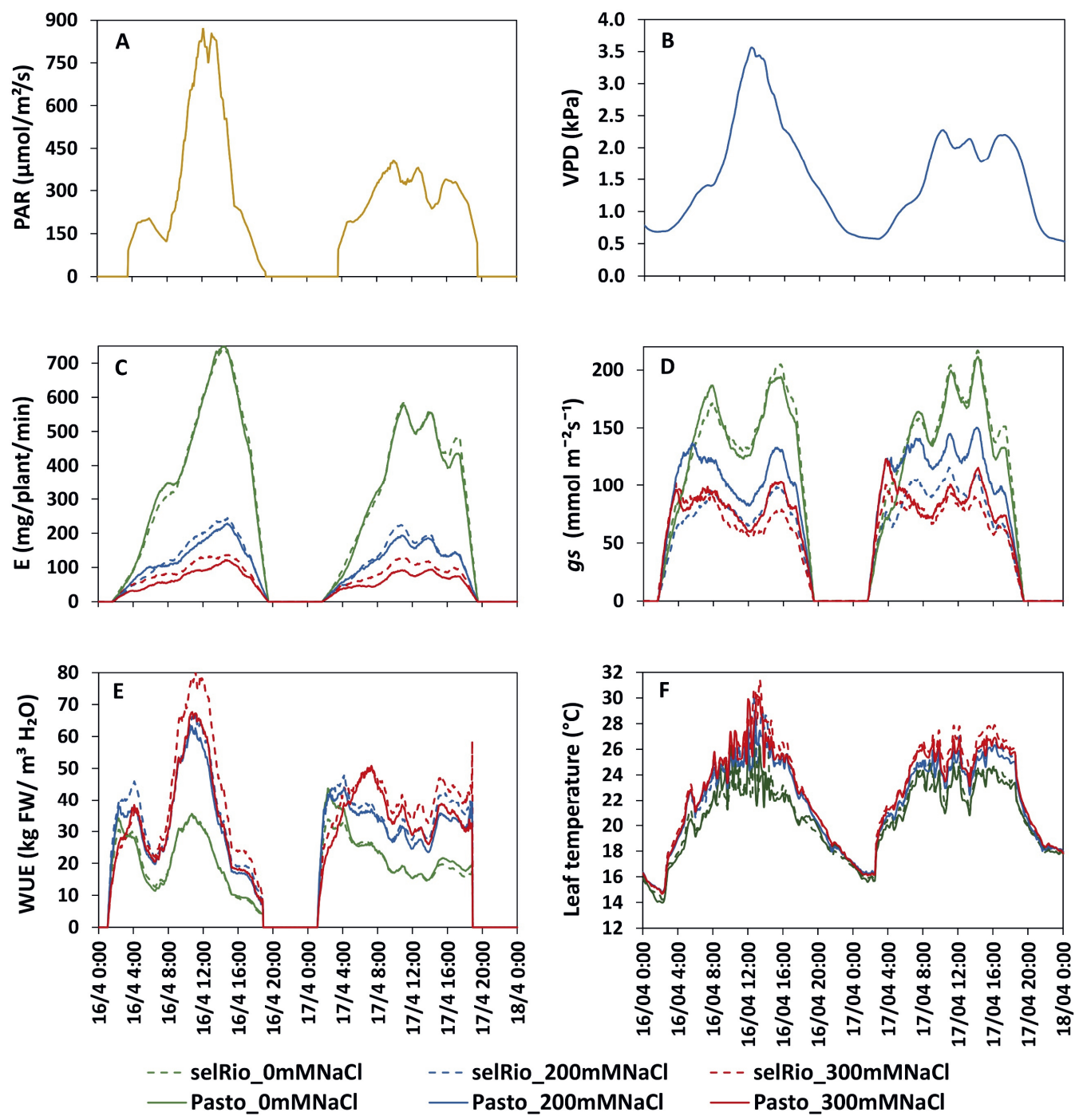

Figure 6. Daily patterns of experimental conditions measured by sensors and physiological components derived from Plantarray measurements. The influence of a bright (16/4/2019, 62 DAS, 41 days after the start of the stress) and cloudy day (17/4/2019, 63 DAS, 42 days after the start of the stress) are compared. A) Light intensity. B) VPD. C) Transpiration rate (E). D) Stomatal conductance (gs). E) Water use efficiency (WUE). F) Leaf temperature measured by a thermal camera. 


\subsection{Effect of salt on the photosynthetic capacity of quinoa}

A rapid light response curve was recorded at 76 DAS to investigate the effect of salt on the photosynthetic capacity of quinoa plants, plotting effective quantum yield $(\varphi P S I I)$ as a function of PAR irradiance (Figure $7 A$ ). $\varphi P S I I$ provides an indication of the amount of energy used for photochemistry. At the lowest level of irradiance, $\varphi P S I I$ has its maximum value, which for control was 0.77 , indicative for a healthy leaf. At 183- $965 \mu \mathrm{mol}$ photons $\mathrm{m}^{-2} \mathrm{~s}^{-1}$, the effect of salt was the most pronounced. For selRiobamba, $\varphi$ PSII was $7 \%$ lower at $200 \mathrm{mM} \mathrm{NaCl}$ and $11 \%$ lower at $300 \mathrm{mM} \mathrm{NaCl}$. Pasto showed a $10 \%$ decrease at $200 \mathrm{mM} \mathrm{NaCl}$ but only a $5 \%$ decrease at $300 \mathrm{mM} \mathrm{NaCl}$. $\varphi P S I I$ multiplied by PAR gives a relative indication of the photosynthetic electron transport rate (ETR) (Figure 7B). Since $\varphi P S I I$ is not linked to the amount of chlorophyll, the calculated parameter is the relative ETR ( $r E T R$ ) and is distinct from the ETR obtained from an oxygen-base P-E curve (Ralph and Gademann 2005). The rETR rapidly increased with light intensity. However, the steady state was not reached with the maximum actinic light applied in this study $\left(1692 \mu \mathrm{mol}\right.$ photons $\left.\mathrm{m}^{-2} \mathrm{~s}^{-1}\right)$. The rETR of plants growing under salt treatment were slightly lower but not significantly different than control plants (Figure 7B). 

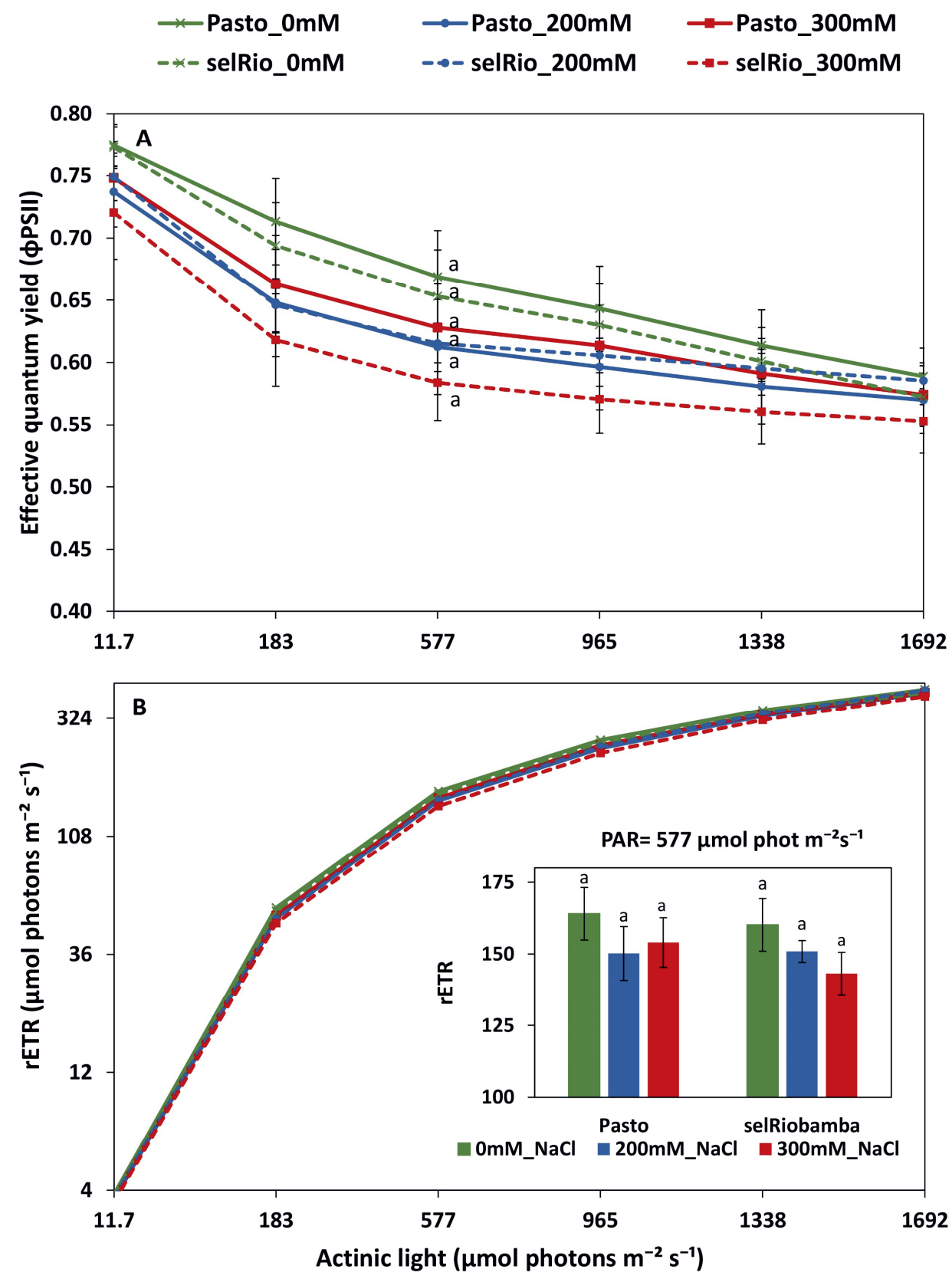

Figure 7. Derived parameters of a rapid light curve from light adapted detached young leaves of quinoa. A) Effective quantum yield as a function of PAR. B) Relative electron transfer rate as a function of PAR. Means of 4 plants. Error bars indicate SE of individual means. Statistically significant differences $(p \leq$ $0.05)$ between any variety and salt treatment combination are shown with different letters. 


\subsection{Functional growth analysis of quinoa}

During the course of this experiment, RGR and its components were monitored in three main periods: before the application of the stress, from the beginning of the stress until the first destructive harvest (36-41 DAS) and between the first and second destructive harvests (47-77 DAS). During the first phase of stress, salt significantly decreased RGR, especially in Pasto (Figure 8A). At $200 \mathrm{mM}$ $\mathrm{NaCl}$, selRiobamba RGR was similar to control, while Pasto's RGR was already significantly lower (Figure 8A). The decrease in the RGR of Pasto at this time appeared to be mostly caused by a significant decrease in the specific leaf area (SLA) (Figure 8B), while the reduction in net assimilation rate (NAR) was mostly responsible for the reduced RGR of selRiobamba (Figure $8 \mathrm{C}$ ). For both varieties, LWR was not significantly different between the control and $200 \mathrm{mM} \mathrm{NaCl}$, but increased in the most severe treatment of $300 \mathrm{mM} \mathrm{NaCl}$ (Figure 8D). During the last period in which RGR analysis was performed (47-77 DAS), the RGR components were less affected by salt. Only the SLA was decreased by the salt treatment and, interestingly, NAR was even higher under salt stress than under control conditions for Pasto.

Radiation use efficiency (RUE) provides a measurement of the efficiency of a plant to use radiation energy for biomass production. In our experiment, the LINTUL crop model was used to estimate RUE as an integration of several physiological parameters. During the first period of growth after the application of the stress (36-41 DAS), RUE was significantly decreased by salt stress, and differences were found between varieties. For Pasto, RUE decreased by $33 \%$ at $200 \mathrm{mM} \mathrm{NaCl}$ and by $73 \%$ at $300 \mathrm{mM} \mathrm{NaCl}$. For selRiobamba, RUE was only decreased at the highest salt concentration of $300 \mathrm{mM} \mathrm{NaCl}$ by $63 \%$ (Figure $8 \mathrm{E}$ ). 

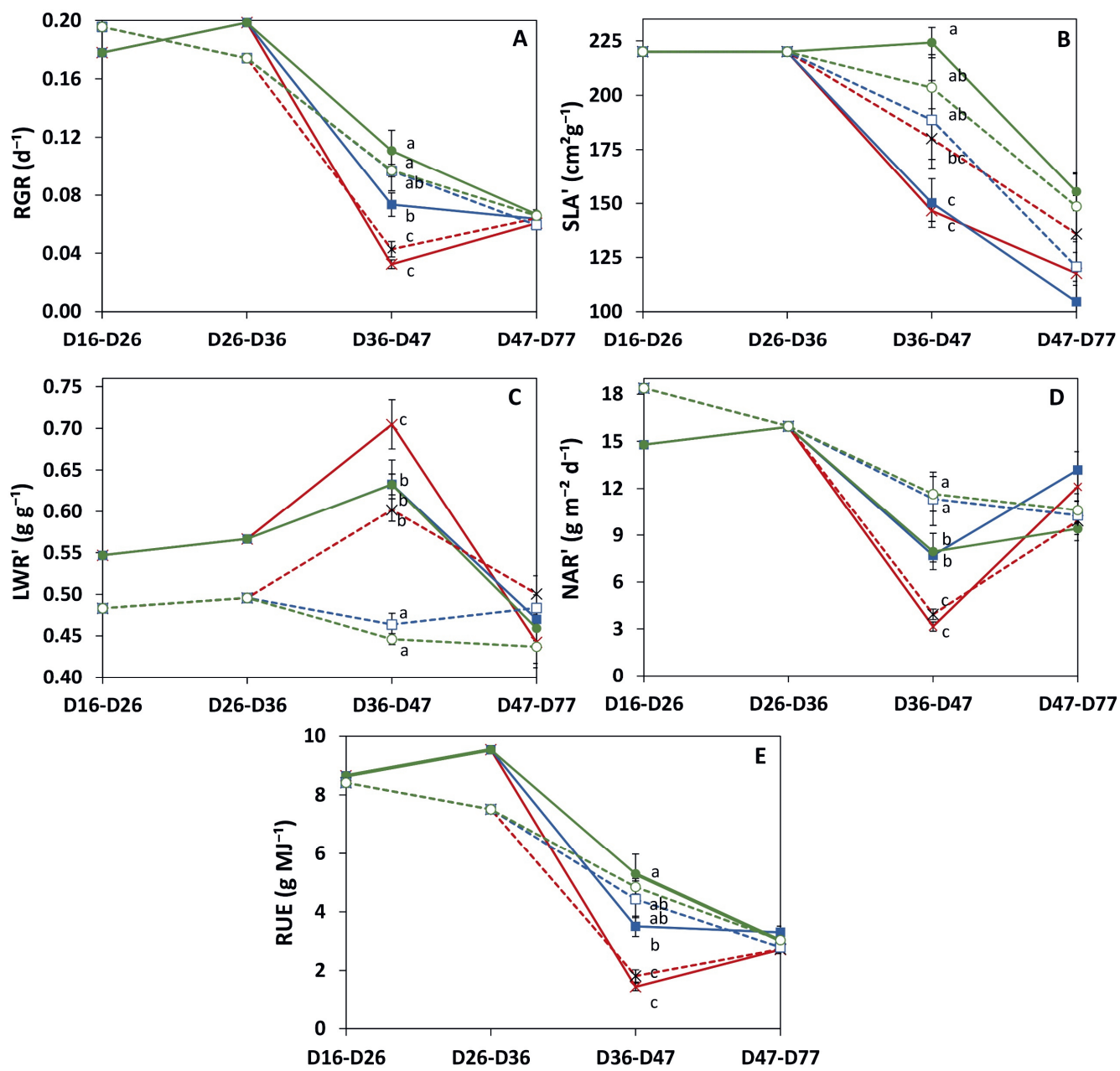

Days after sowing

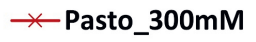

$\rightarrow-$ Pasto_200mM

$\longrightarrow$ Pasto_OmM

--*--selRio_300mM

-- ---selRio_200mM

--o--selRio_OmM

Figure 8. Relative growth components of quinoa throughout the growing period. A) Relative growth rate (RGR). B) Incremental specific leaf area (SLA'). C) Incremental leaf weight ratio (LWR'). D) Incremental net assimilation rate (NAR'). E) Radiation use efficiency estimated by LINTUL mechanistic model. Means of 4 plants. Error bars indicate SE of individual means. Statistically significant differences $(p \leq 0.05)$ between any variety and salt treatment combination are shown with different letters. 


\section{Discussion}

We used the Plantarray phenotyping platform to gain insight in the salt response of two quinoa varieties, and in the consequences of different strategies with respect to transpiration, assimilation and growth. The impact of salt stress on the growth and physiological responses of the quinoa varieties Pasto and selRiobamba was similar as reported before (Jaramillo Roman et al. 2020). The plants remained green and were able to grow under salinity but dry biomass was strongly reduced by on average $44 \%$ at $200 \mathrm{mM} \mathrm{NaCl}$ and $66 \%$ at $300 \mathrm{mM}$ after six weeks of the start of the salt treatment. Pasto and selRiobamba showed differences in their physiological responses to salinity, which resulted in a higher salt tolerance in selRiobamba than Pasto. The main cause of the reduced growth rate of Pasto was a decrease in SLA, which strongly indicates that the leaf area expansion rate of Pasto was relatively low and leaf thickness increased. The SLA of selRiobamba was less affected; the main cause of the reduction of the growth rate of this variety under salinity appeared to be a lowered NAR, which is indicative of the photosynthetic capacity of the plant (Lambers and Poorter 1992). The varieties also differed in ion uptake and distribution within plant tissues. In Pasto, the $\mathrm{Na}^{+}$and $\mathrm{Cl}^{-}$concentration in young leaves remained lower than the root medium, while in selRiobamba concentrations of $500 \mathrm{mM}$ were measured for both ions, which points to a stronger shoot ion exclusion activity for Pasto. As reported before, quinoa is recognized for its ability to retain or even increase $\mathrm{K}^{+}$under salinity, especially in young photosynthetically active leaves (Hinojosa et al. 2018; Jaramillo Roman et al. 2020). Pasto and selRiobamba showed differences in $\mathrm{K}^{+}$retention. In Pasto, $\left[\mathrm{K}^{+}\right]$in young leaves of salt stressed plants was $400 \mathrm{mM}, 20 \%$ higher than in plants under control conditions, while in selRiobamba, $\left[\mathrm{K}^{+}\right]$was $50 \%$ reduced under the $300 \mathrm{mM} \mathrm{NaCl}$ treatment. The energetic cost of $\mathrm{K}^{+}$retention under saline conditions is high: 1-2 mol ATP is needed for the retention of $1 \mathrm{~mol}$ of $\mathrm{K}^{+}$(Rubio et al. 2020). For this reason, retaining $\mathrm{K}^{+}$under salinity has been described as a 'metabolic switch', in which a larger amount of ATP is redirected to adaptive traits to salt stress (Rubio et al. 2020). Our results indicate that Pasto allocated more resources towards this adaptation, but this may have come at a metabolic cost, reflected in the higher reduction of biomass under salt stress (Figure 2).

\subsection{Whole-plant adaptations to salt stress}

Transpiration was strongly reduced by salinity. Under the $200 \mathrm{mM} \mathrm{NaCl}$ treatment, cumulative transpiration was $60 \%$ reduced for both varieties. The 300 $\mathrm{mM} \mathrm{NaCl}$ treatment strengthened this reduction and the differences between 
varieties; cumulative transpiration was $66 \%$ and $80 \%$ reduced in Pasto and selRiobamba, respectively. Cumulative transpiration had a strong positive correlation with the fresh weights of the plants (Figure 9A). However, under $200 \mathrm{mM}$ $\mathrm{NaCl}$, Pasto had a stronger reduction in biomass than selRiobamba while transpiration was similarly reduced. It is possible that while the available resources (water, $\mathrm{CO}_{2}$ ) in both varieties were similar, assimilates were less allocated to biomass production in Pasto, and more directed towards salt tolerance responses (morphological adaptations like decrease in SLA, $\mathrm{Na}^{+}$and $\mathrm{Cl}^{-}$exclusion, $\mathrm{K}^{+}$ retention, among others). Transpiration was significantly correlated to $\left[\mathrm{Na}^{+}\right]$and $\left[\mathrm{Cl}^{-}\right]$ in the roots (Figure 9B) but not in young leaves. In addition, $\mathrm{Na}^{+}$and $\mathrm{Cl}^{-}$ concentrations in leaves of Pasto were lower than selRiobamba, while the transpiration rate in both varieties was similar. This indicates that the ion concentrations in young leaves may be more determined by ion exclusion mechanisms (mainly at xylem loading) than by the transpiration rate of the plants. We examined the effect of the reduction of transpiration on the growth rate and the RGR components. Despite the $60 \%$ reduction in transpiration, the $200 \mathrm{mM} \mathrm{NaCl}$ treatment did not affect NAR, which means the photosynthetic rate was not affected by this for quinoa mild salinity level. Transpiration had a positive correlation with SLA (Figure 9D). The morphological adaptation of reduced leaf expansion and thicker leaves reduced the total surface available for water loss, which agrees with the lower transpiration of Pasto, especially under $300 \mathrm{mM} \mathrm{NaCl}$. 

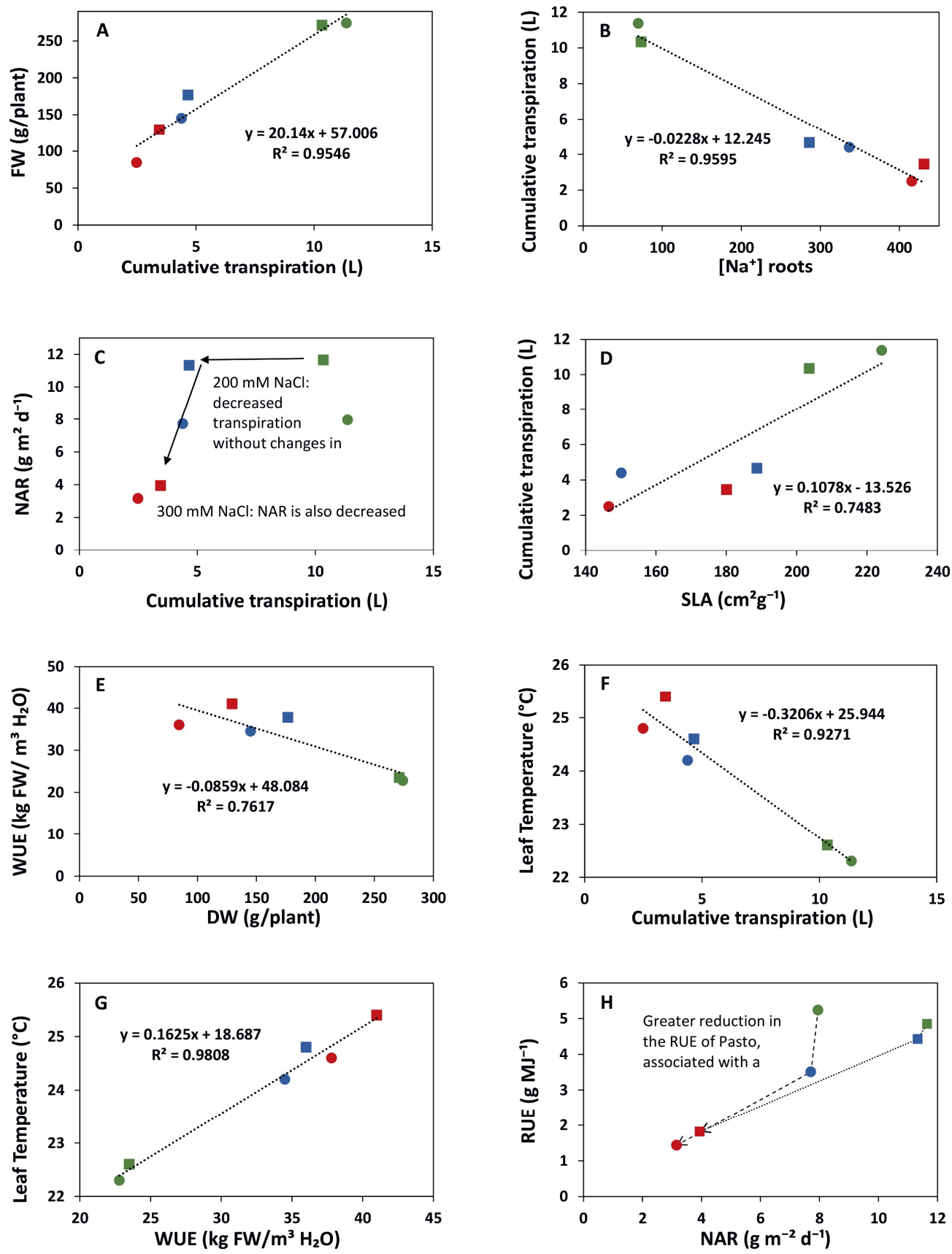

Figure 9. Relations between some of the physiological traits analysed in this study. The values of selRiobamba are indicated by squares and of Pasto by circles. Level of stress is indicated by colour; green: no-salt, blue: $200 \mathrm{mM} \mathrm{NaCl}$, red: $300 \mathrm{mM} \mathrm{NaCl}$. 
Interestingly, selRiobamba had a smaller reduction in total plant transpiration than Pasto, but its stomatal conductance (transpiration per unit leaf area, standardized for VPD) was more reduced than that of Pasto. This may be explained by the stronger reduction in leaf expansion of Pasto. We argue that this might be an important difference between the salt stress response of these quinoa varieties. Pasto lowers total transpiration by a decreased leaf area (without strong control of stomata) while selRiobamba appears to control stomatal aperture to minimize water loss and optimize transpiration.

The effect of salinity on the stomatal conductance calculated from Plantarray data $\left(g s_{\text {system }}\right)$ was comparable to the effect on the stomatal conductance measured with a porometer ( $g s_{\text {porometer }}$ (Figure 4$)$, validating the $g s_{\text {system }}$ calculations. However, the $g s_{\text {system }}$ values were approximately $46 \%$ lower than the $g s_{\text {porometer }}$ values under all treatments. This seems counterintuitive as the $g s_{\text {system }}$ was derived from the total leaf transpiration (transpiration from both the abaxial and adaxial side of leaves),

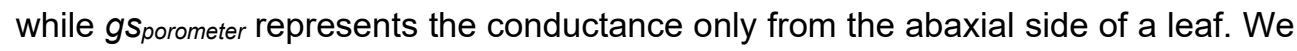
tested the relative contribution of abaxial and adaxial stomatal conductance to the total stomatal conductance with plants grown at 0 and $300 \mathrm{mM} \mathrm{NaCl}$ in a separate experiment. At $0 \mathrm{mM}$, the adaxial gs was not significantly different from the abaxial gs; the ratio of adaxial to abaxial gs was 1.02 (Supplementary Table 1). At $300 \mathrm{mM}$, however, this ratio was much lower than $1(0.76)$. We used this information to correct the whole plant $g s$ calculated from porometer $g s$ values for the relative contribution of the abaxial and adaxial sides of the leaves. Another parameter that needs to be considered when comparing $g s_{\text {porometer }}$ and $g s_{\text {system }}$ is the boundary layer resistance. The $g s_{\text {porometer }}$ data is not affected by the boundary layer resistance (McDermitt 1990). Yet the influence of boundary layer resistance in plants growing in the greenhouse might considerably decrease whole-plant conductance (Katsoulas et al. 2007), and the effect of the boundary layer resistance $(g b)$ is not considered in the calculation of the $g s_{s y s t e m}$. The $g s_{s y s t e m}$, which in fact is the total conductance, includes $g s$ as well as $g b$, and is equal to $1 /(1 / g s+1 / g b)$. Here, $g s$ is the stomatal resistance from both sides of the leaves combined and $g b$ is the boundary layer resistance. Therefore, the whole plant gs was used to estimate a single boundary layer resistance for this experiment $\left(230 \mathrm{mmol} / \mathrm{m}^{2} / \mathrm{s}\right)$. The boundary layer resistance in greenhouses varies from $200-2000 \mathrm{mmol} / \mathrm{m}^{2} / \mathrm{s}$ depending on the wind speed and the size of leaves (Kimura et al. 2020). The $g b$ calculated in our experiment corresponds to a very low wind speed of $0.05 \mathrm{~m} / \mathrm{s}$, which is agreement with the conditions in our greenhouse. The $g s_{s y s t e m}$ values corrected for the boundary layer resistance 
( $\left.g s_{\text {sysyem_corr }}\right)$ are highly comparable to $g s_{\text {porometer }}$ values corrected for both sides of the leaf (gsporometer_corr) (Figure 10).

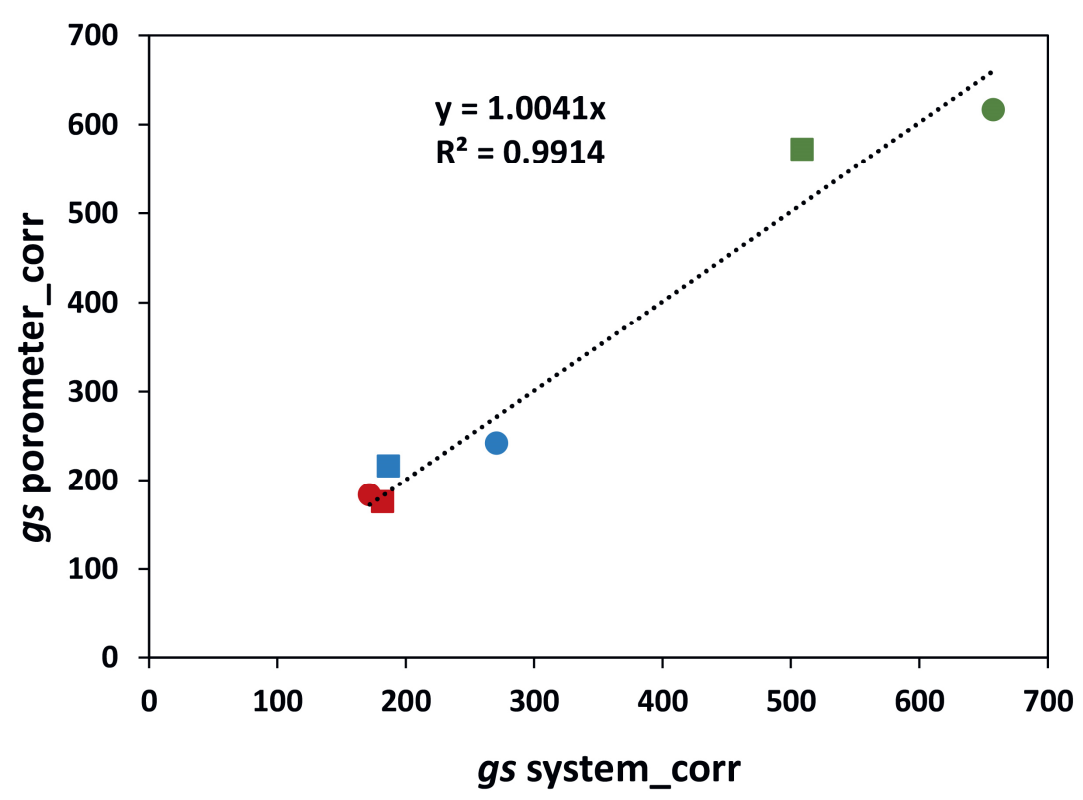

Figure 10. Correlation between gs porometer measurements corrected for the abaxial and adaxial sides of the leaves ( $g s_{\text {porometer_corr) }}$ and the $g s$ from the system after correcting for the effect of the boundary layer resistance ( $g s_{\text {sysyem_corr }) \text {. }}$

WUE can be defined and measured in different ways; it can be an instantaneous measurement of the ratio of the photosynthetic rate and the transpiration rate, or a productivity measurement of the ratio of biomass accumulation and water use over a period of time (Leakey et al. 2019). The Plantarray system provides a platform to continuously monitor changes in WUE that result from dynamic interactions between water use and biomass gain by the plants. Thus, WUE can be studied as a dynamic process more than a productivity indicator. In our study, salinity significantly increased the WUE of quinoa. It should be noted that the WUE calculated from Plantarray data is expressed as $\mathrm{kg}$ Fresh Weight/ $\mathrm{m}^{3} \mathrm{H}_{2} \mathrm{O}$, and not dry weight. However, the differences in WUE caused by the salt treatment in quinoa are not a result of a lowered water content in the leaves of salt stressed plants, since the differences in the dry matter content between treatments are negligible. A main goal of breeding for salt or drought stress tolerance is to improve the WUE of plants, but only as long as this also supports greater productivity under the stress conditions (Leakey et al. 2019). High values of 
WUE are typically observed when stomatal conductance is lower that the potential maximum for a genotype, which also results in reduced growth (Yoo et al. 2009). Therefore, higher values of WUE are often associated with smaller plants, lowered growth and low crop production (Blum 2009). By continuously monitoring WUE and parameters that might affect the WUE (evaporation, transpiration rate, biomass gain, stomatal conductance, leaf area, and environmental parameters such as VPD) we might be able to identify whether the increase of WUE in a particular genotype is mostly associated with lower water loss, or whether certain adaptations contribute to increase productivity and maximizing the efficiency of water use. Both Pasto and selRiobamba showed higher WUE under salinity; however, with similar amounts of water transpired, more biomass was produced by selRiobamba (Figure 9E). The increased WUE might therefore be a favourable trait for the productivity of selRiobamba, and more of a water-saving strategy for Pasto; the causes of the increase of WUE in both varieties might be associated with different physiological mechanisms that should be further explored.

One of the greatest advantages of the Plantarray system is the temporal resolution of the measurements that enables to monitor water use responses not only to environmental variation throughout the growing cycle, but also to diurnal variations of environmental parameters like light irradiance and atmospheric VPD. The day-to-day patterns of transpiration, gs and WUE were compared between two consecutive days that showed different levels of VPD and light irradiance (Figure 6). The relative differences in the transpiration rate between days were higher under control than under salt stress. Reducing transpiration is a common adaptation to saline conditions. Photosynthesis is primarily limited by the $\mathrm{CO}_{2}$ uptake, while it is affected by light availability to a much lower extent (Flexas et al. 2004). Assuming the photosynthetic machinery is saturated, the additional water transpired under control conditions in a day with higher irradiation will be either wasted or used for canopy cooling purposes, but will not be associated with higher biomass synthesis. Under saline conditions, the plant cannot afford to waste water. Therefore, a tighter control of transpiration rate by quinoa under salt stress is necessary. Stomatal conductance was influenced by the fluctuations in VPD during the day, which has been identified as a "patchy" stomatal behaviour (Buckley 2005). The daily gs is depressed at maximal VPD (midday), and a high gs is observed in the early morning, when light irradiance increases and VPD is still low (Gosa et al. 2019). When VPD is high, evaporation from the leaves is high as well, so a strict control of stomatal opening at high VPD might be an additional strategy to enhance $\mathrm{CO}_{2}$ uptake without excessive loss of water. The continuous monitoring of $g s$ indicated that quinoa has 
a strict control in stomatal opening, which might be even increased under salinity. However, the total amount of water that could be saved throughout the growth cycle by the temporal control of stomatal opening needs to be estimated.

Infrared thermography was used to monitor salinity-induced changes in leaf temperature. Leaf temperature has been considered a proxy for gs (Hackl et al. 2012; Ivushkin et al. 2018), and canopy thermography was also used as an indicator of salinity stress in quinoa (Ivushkin et al. 2019a). The surface of a leaf is cooled by evaporation, so a strong correlation exists between the cooling of the leaves with transpiration rate and stomatal opening. In our study, canopy temperature had a strong negative correlation with gs, transpiration, biomass, and growth, and a positive correlation with water use efficiency, $\mathrm{Na}^{+}$and $\mathrm{Cl}^{-}$content in young leaves (Figures $10 \mathrm{~F}-\mathrm{G}$ ). Salinity significantly increased the leaf temperature by $2{ }^{\circ} \mathrm{C}$ at 200 $\mathrm{mM} \mathrm{NaCl}$ and $2.7^{\circ} \mathrm{C}$ at $300 \mathrm{mM} \mathrm{NaCl}$. Salinity significantly increased the leaf temperature by $2{ }^{\circ} \mathrm{C}$ at $200 \mathrm{mM} \mathrm{NaCl}$ and $2.7^{\circ} \mathrm{C}$ at $300 \mathrm{mM} \mathrm{NaCl}$. Genotype-specific responses could also be identified using infrared thermography (Figure 5). The leaf temperatures of selRiobamba were slightly but not significantly higher than Pasto. Previously, we pointed out that Pasto had a lower transpiration rate than selRiobamba, and suggested that this was achieved by decreasing SLA rather than decreasing gs. An additional advantage of this adaptation could be that water loss is reduced without compromising the cooling system of leaves. In this way, significant differences were found in the transpiration rate and gs of Pasto an selRiobamba, without significant differences in leaf temperatures. Based on our measurements, leaf temperature has the potential to be used as a proxy to gs, also to WUE; the diurnal pattern of WUE was very well followed by the leaf temperature pattern, and both traits were highly correlated $(r=0.99)$.

Reduced stomatal conductance under salinity stress is an important determinant for reduced photosynthetic activity. However, other non-stomatal photosynthesis-limiting factors might also play a role when plants face salt stress. Chlorophyll fluorescence was used to measure the response of photosynthetic parameters to salinity. Rapid light curves provide information on the saturation characteristics of electron transport, as well as the overall photosynthetic performance of a plant over a wide range of ambient light intensities (Ralph and Gademann 2005). It was previously reported that net apparent photosynthesis activity $\left(A_{N}\right)$ and the internal $\mathrm{CO}_{2}$ concentration at PAR levels higher than $500 \mu \mathrm{mol}$ photons $\mathrm{m}^{-2} \mathrm{~s}^{-1}$ were significantly reduced in quinoa by a salt treatment of $250 \mathrm{mM}$ $\mathrm{NaCl}$, while photochemical parameters, light compensation point and maximum apparent photosynthetic quantum yield were not affected (Becker et al. 2017). In our 
experiment, $\varphi$ PSII and rETR as a function of irradiance were more impacted by salt treatment than the Fv/Fm ratio of dark-adapted leaves, but no significant differences were found in these parameters between treatments. However, at lower light levels in the range of 183 to $965 \mu \mathrm{mol}$ photons $\mathrm{m}^{-2} \mathrm{~s}^{-1}$ the maximum rate of photosynthesis was lowered (similar to the LICOR measurements by Becker et al. (2017)). In a crop situation, most leaves are exposed to medium light levels, which means that a stronger effect of salt (at 200 and $300 \mathrm{mM} \mathrm{NaCl}$ ) on maximal the photosynthesis rate may be experienced. Even though the $\varphi P S I I$ was not significantly different between treatments and varieties, it was slightly lower for selRiobamba than Pasto, and Pasto had a higher $\varphi$ PSII under $300 \mathrm{mM} \mathrm{NaCl}$ than $200 \mathrm{mM} \mathrm{NaCl}$. This suggests and additional adaption of Pasto to salt stress to reduce non-photochemical quenching at the higher salinity level. It is possible that the higher $\varphi$ PSII of Pasto is also related to its lower SLA. Thicker leaves likely have a higher density of Rubisco and chlorophyll per unit of leaf area, thus their photosynthesis rate might increase (Terashima et al. 2011).

\subsection{Varietal differences in responses to salt stress from a resource use perspective}

The differences in morphological and physiological traits associated with the use of water, energy and assimilates of Past and selRiobamba in response to salinity indicate that these varieties have different strategies to cope with salt stress.

The adaptations to stress of Pasto point to a "conservative-growth" strategy (Sade et al. 2012). Pasto had a stronger decline in SLA ( 35\%), and a higher reduction in transpiration rate than selRiobamba. The higher WUE was associated with less water loss, but resulted in higher reduction in biomass relative to selRiobamba that had higher RUE and RGR. Pasto also had a high rate of $\mathrm{Na}^{+}$ion exclusion from photosynthetically active leaves, accompanied by high $\mathrm{K}^{+}$levels. These adaptations were more pronounced and clearly different from selRiobamba at $300 \mathrm{mM} \mathrm{NaCl}$. SelRiobamba on the other hand appeared to follow a more "acquisitive-growth" strategy (Reich et al. 2003). Transpiration was reduced by salinity but less than Pasto, while it had higher $\mathrm{Na}^{+}$and $\mathrm{Cl}^{-}$contents in the shoots. The conservative growth response of Pasto serves to protect tissues to prolonged and severe salinity and Pasto would therefore be better able to survive such conditions than selRiobamba. However, under the conditions used in this study, selRiobamba outperformed Pasto in terms of growth and yield at both salt concentrations. SelRiobamba thus appears to be able to maintain its growth rate better under mild or relatively short-term salt stress. 
In chapter 2, we evaluated quinoa varieties including Pasto and selRiobamba under mild as well as severe salinity, and our results suggested that the choice of a conservative or acquisitive growth strategy of a variety is influenced by the severity and duration of the stress in addition to a genetic component (Jaramillo Roman et al. 2020). The ability to adapt strategies is to some extent also reflected in the results in this study; while at $200 \mathrm{mM} \mathrm{NaCl}$ Pasto's transpiration rate was similar to selRiobamba, the more severe stress changed its behaviour to a more conservative growth strategy. Possibly, quinoa can be a facultative halophyte because of the ability to switch from an acquisitive growth to a conservative growth strategy when stress becomes severe and less resources are available, and Pasto and selRiobamba differ in the salinity threshold that flips this switch. Accurate and high-throughput phenotyping platforms like the Plantarray system are highly useful tools to distinguish physiological differences between both strategies, and to identify genetic variation that can be used to improve quinoa yields in a broad range of saline environments.

\subsection{Future perspectives of functional phenotyping in abiotic stress tolerance research}

Several reports have examined the influence of salt stress on physiological parameters related to water and carbon fluxes, photosynthesis and ion contents in quinoa. However, extrapolating data from limited time-point measurements on single leaves (e.g. gas exchange rate, rate of net photosynthesis) to the growth cycle of a crop is not straightforward (Harris et al. 2010; Morton et al. 2019). This study shows the potential of the implementation of high throughput functional phenotyping in the understanding of complex physiological responses to salt stress. The Plantarray system is scalable, which means that a high number of plants and genotypes could potentially be screened simultaneously. The possibility to scan entire mapping populations opens up the possibility of identifying genetic determinants underpinning differences in traits such as water use efficiency or the diurnal control of stomatal conductance. The system can for instance be complemented with digital imaging systems that monitor leaf expansion and estimate leaf area, which will produce even more accurate measurements of stomatal conductance. In addition, the highresolution continuous monitoring of growth and transpiration provides valuable data for the development and improvement of crop growth models. We used the mechanistic crop model LINTUL to integrate several physiological processes for an estimation of the radiation use efficiency (RUE) and to analyse the impact of salinity on the RUE of quinoa (Sinclair and Muchow 1999; Spitters and Schapendonk 1990). We estimated average RUE values of $5 \mathrm{gDW} / \mathrm{MJ}$ under control conditions. Previous 
studies in quinoa reported a significantly lower RUE (1.4- 1.75 gDW/MJ) (Razzaghi et al. 2012; Ruiz and Bertero 2008). This difference may be attributed to different growing conditions and the time of the measurements. High RUE values (3-5 gDW/MJ) are often reported in plants growing under controlled conditions due to the high proportion of diffuse radiation inherent to glasshouses and the lower daily incident radiation that might induce higher photosynthetic efficiency (CabreraBosquet et al. 2016). In our conditions, RUE was significantly reduced by salt stress, which is in contrast with previous studies in which no differences in RUE were found even with a salt treatment of $40 \mathrm{dS} / \mathrm{m}$ (Razzaghi et al. 2012). Razzaghi et al. (2012) estimated RUE in a field trial where the average light level experienced by the leaves is much lower. In those conditions the initial light use efficiency determines RUE. In our conditions, where the LAI remained much lower than in a crop situation, most leaves experienced higher light levels than in a crop. This will explain the lower overall RUE estimated under stress conditions. Although genetic improvement of RUE has been suggested as a way to increase yield, few studies have explored its genetic variation, probably due to technical difficulties in the estimation of this parameter (Cabrera-Bosquet et al. 2016). Our results suggest that using the Plantarray system data as input for growth models may be a viable strategy for crop improvement based on RUE. High throughput and high-resolution technologies thus enable the dissection of plant growth and water consumption into very specific parameters that could constitute novel targets for the improvement of abiotic stress tolerance of crops.

\section{Acknowledgements}

This study was conducted as a pilot experiment during the development phase of the Netherlands Plant Eco-phenotyping Centre (NPEC). We acknowledge Tom P.J.M. Theeuwen and Ferdinand Diesman for their expert advice in the measurements with the Robin PSI PlantScreen ${ }^{T M}$. We also gratefully acknowledge the assistance of Sean Geurts and Andre Maassen in the greenhouse. Viviana Jaramillo is financially supported by a scholarship from the Secretariat for Higher Education, Science, Technology and Innovation of the Republic of Ecuador (Contract Number 2015-AR2Q8878). 


\section{Supplementary material}
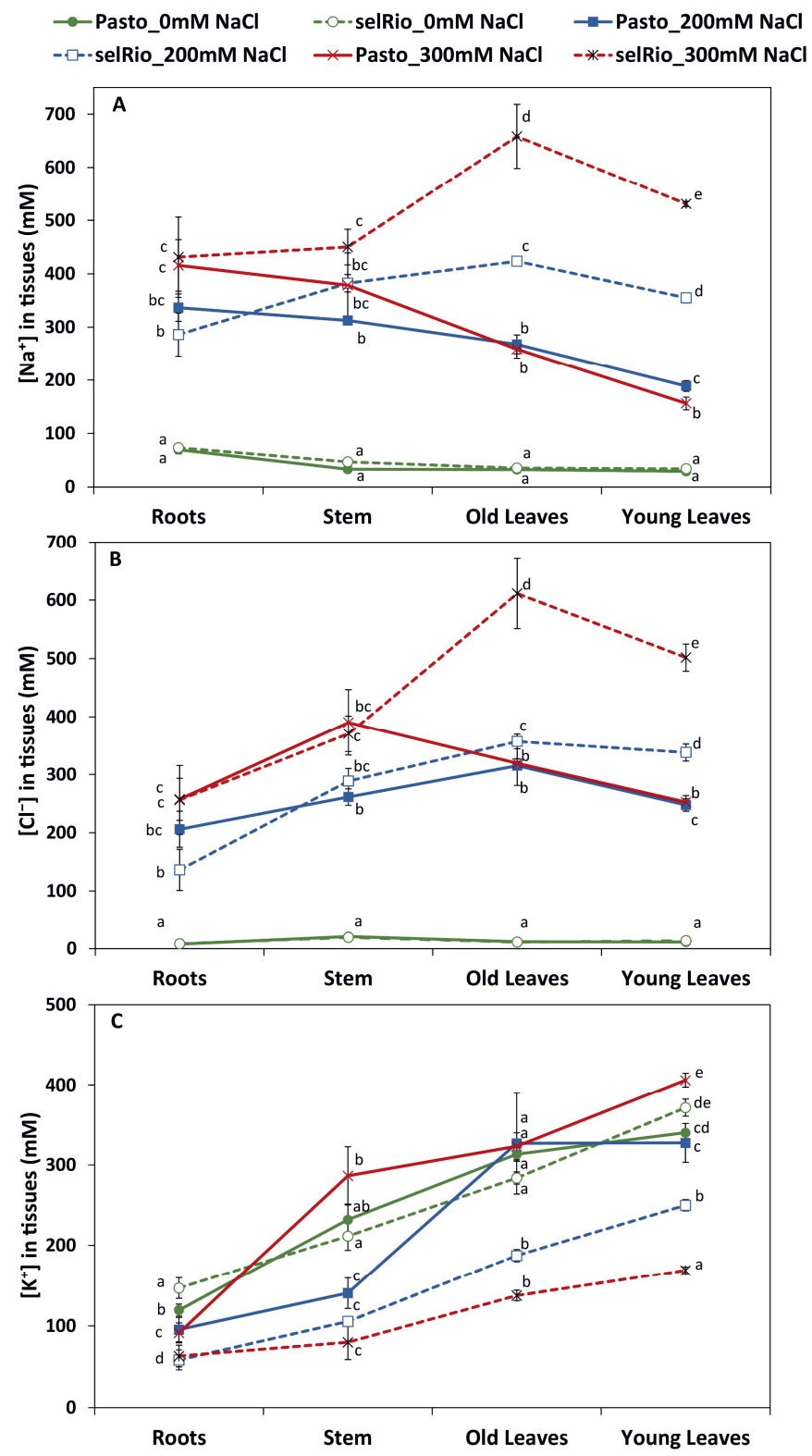

Figure S1. lon contents in tissues of quinoa at different levels of salinity 77 DAS (41 days after the start of salt stress). A) $\left[\mathrm{Na}^{+}\right]$in different tissues of quinoa at different levels of salinity. $\mathrm{B}$ ) $\left[\mathrm{Cl}^{-}\right]$in different tissues of quinoa at different levels of salinity. C) $\left[\mathrm{K}^{+}\right]$in different tissues of quinoa at different levels of salinity.Means of 4 plants. Error bars indicate SE of individual means. Statistically significant differences $(p \leq 0.05)$ between any variety and salt treatment combination (within each tissue) are shown with different letters. 
Table S1. Comparison between gs measured with a porometer in the abaxial and the adaxial side of leaves of quinoa plants growing under control conditions $(0 \mathrm{mM} \mathrm{NaCl})$ or under salt treatment $(300 \mathrm{mM}$ $\mathrm{NaCl}$ ) at 64 DAS (35 days after the start of salt treatment). Means of 6 plants.

\begin{tabular}{|c|c|c|c|c|}
\hline \multirow{2}{*}{$\begin{array}{l}\text { Variety } \\
\text { Pasto }\end{array}$} & \multicolumn{2}{|c|}{ Salt treatment gs abaxial $\left(\mathrm{mmol} \mathrm{m}^{-2} \mathrm{~s}^{-1}\right)$} & \multicolumn{2}{|c|}{ gs adaxial $\left(\mathrm{mmol} \mathrm{m}^{-2} \mathrm{~s}^{-1}\right)$ ratio gs adaxial/gs abaxial } \\
\hline & $0 \mathrm{mM} \mathrm{NaCl}$ & 185 & 189 & 1.02 \\
\hline & $300 \mathrm{mM} \mathrm{NaCl}$ & 84.6 & 63.3 & 0.75 \\
\hline selRiobamba & $0 \mathrm{mM} \mathrm{NaCl}$ & 188 & 192 & 1.02 \\
\hline & $300 \mathrm{mM} \mathrm{NaCl}$ & 93.3 & 72 & 0.77 \\
\hline
\end{tabular}





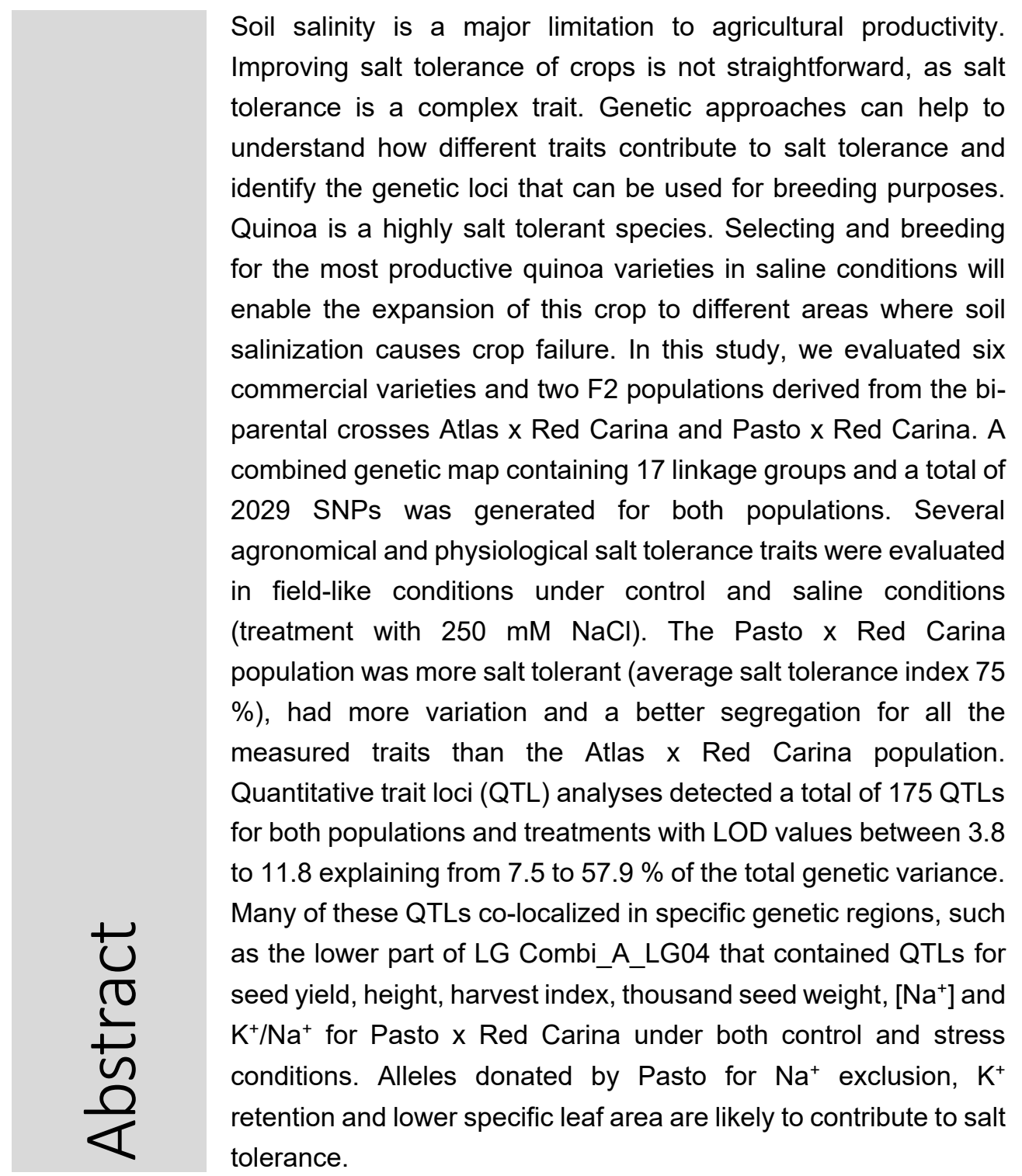




\section{Introduction}

Quinoa (Chenopodium quinoa) is a highly nutritious food crop that has gained international attention due to the nutritional properties of its seeds, its high genetic diversity and its adaptability to a wide range of harsh environments (ZuritaSilva et al. 2014). Originating from the Andean region of South America, quinoa has adapted to grow in diverse soils and environmental conditions, which explains its resilience to frost, drought, a wide range of temperatures and soil salinity (LópezMarqués et al. 2020). Soil salinity is a major abiotic threat to agriculture that affects more than $6 \%$ of the world surface and is expected to increase as a result of climate change and poor agricultural practices (Okur and Örçen 2020). To meet this challenge, agricultural production can benefit from the incorporation of more resilient crops, such as quinoa, or from the improvement of the salt tolerance of the actual major crops (Roy et al. 2014).

Quinoa can tolerate a wide range of soil salinity. It grows well at salt levels that are detrimental for most other crops (100-200 $\mathrm{mM} \mathrm{NaCl})$, while it can also survive salt levels equal or even higher than those of seawater (>400 $\mathrm{mM} \mathrm{NaCl})$ (Hinojosa et al. 2018). Previously, we demonstrated that quinoa utilizes salt exclusion strategies (from roots and shoots) to grow under mild salinity (100-200 mM $\mathrm{NaCl}$ ) or short-duration stress, while accumulating and tolerating ions in tissues enables the plants to survive severe $(400 \mathrm{mM} \mathrm{NaCl})$ and prolonged salinity (Jaramillo Roman et al. 2020). Few studies have also evaluated the salt tolerance of quinoa under field conditions and found that the reduction of seed yield due to salinity (EC $20 \mathrm{dS} / \mathrm{m}$ ) varied between genotypes and locations and ranged from 15 to $48 \%$ (Hussain et al. 2018; Pulvento et al. 2012). However, the genetics underlying the salt tolerance mechanisms employed by quinoa remain largely unknown.

The genetic underpinning of salt tolerance traits is essential to increase the ability of crops to maintain growth and productivity in saline soils (Roy et al. 2014). By using genetic mapping of quantitative trait loci (QTLs) associated with desired traits, favourable alleles can be incorporated in breeding programs through MarkerAssisted Selection (MAS) (Yamaguchi and Blumwald 2005). While many successful examples are reported on the use of marker-assisted breeding for traits determined by one or only few major gene(s), much less progress is mentioned on the application of MAS to improve abiotic stress tolerance, including salt tolerance (Gilliham et al. 2017). Research on the physiology of salt tolerance has demonstrated that it is a complex trait determined by a number of sub-traits and a number of genes (Flowers 2004). Several QTLs have been reported for salinity 
tolerance and related traits in most of the major crops. Selected traits include plant survival under saline conditions, relative water content, proline content (Fan et al. 2015) and salinity-induced leaf injury (Zhou et al. 2012) in barley; shoot growth, $\mathrm{Na}^{+}$ exclusion and $\mathrm{K}^{+}$accumulation in bread wheat (Asif et al. 2018); $\mathrm{Na}^{+}$exclusion in durum wheat (Munns et al. 2012); plant height in maize (Luo et al. 2017), and survival, $\mathrm{Na}^{+}$and $\mathrm{K}^{+}$content in leaves and roots in rice (Lin et al. 2004). However, very few of these QTLs have been introduced in breeding programs to improve the salt tolerance of these crops. Some successful exemptions are the incorporation of $\mathrm{Na}^{+}$-exclusion related $\mathrm{TmHKT1;5}$ alleles through marker assisted selection that improved the salinity tolerance of durum wheat lines (Munns et al. 2012) and the selection of the Saltol QTL that enhanced the salt tolerance in rice through unloading $\mathrm{Na}^{+}$from the xylem and possibly encodes for an HKT1;5 allele (Thomson et al. 2010).

Despite the agronomic potential and resilience of quinoa, it can still be considered an underutilized and understudied crop, with relatively few active breeding programs. Breeding efforts and the development of genetic tools are needed to enhance our knowledge of the genetics behind the physiological variation for salt tolerance in quinoa, and to improve the crop for several agronomical traits, which is needed to expand and strengthen its expansion to a global scale (GomezPando et al. 2019). Genetic mapping of quinoa has been developed using AFLP markers, array-platform markers and SNPs (Matanguihan et al. 2015). In recent years, several drafts of its genome have been published (Jarvis et al. 2017; Yasui et al. 2016; Zou et al. 2017), which facilitate the identification of molecular markers and the development of molecular breeding tools towards the unravelling of quinoa's genetic diversity.

Here, we screened two quinoa F2 mapping populations plus six European commercial varieties under control conditions and a salt treatment of $250 \mathrm{mM} \mathrm{NaCl}$ (half strength seawater). The objectives of this study were to: i) investigate salt tolerance mechanisms of commercial quinoa varieties cultivated under field-like conditions, ii) evaluate the genetic diversity and segregation of salt tolerance traits in the quinoa mapping populations, iii) explore the genetics underpinning salt tolerance traits in quinoa, and iv) identify QTLs for agronomical traits and salt tolerance traits that might be incorporated into breeding programs of quinoa and possibly allow the discovery of new genes to be used for the improvement of salt tolerance in other species. 


\section{Materials and Methods}

\subsection{Plant materials}

Six European commercial quinoa varieties were evaluated in this study. The varieties Jessie, Dutchess, Pasto, Atlas and Red Carina were developed at Plant Breeding, Wageningen University \& Research (The Netherlands), and the variety Titicaca was developed in the University of Copenhagen (Denmark). In addition, two F2 mapping populations were included. The Atlas $x$ Red Carina mapping population (hereafter referred to as $\mathrm{AxRC}$ ) was evaluated previously for salt tolerance traits (Ivushkin et al. 2019a). Forty progeny lines were selected from this trial based on contrasting responses for the salt tolerance traits $\mathrm{Na}^{+}$and $\mathrm{K}^{+}$content in the shoot, stomatal conductance and seed yield. The second mapping population consisted of 80 F2 progeny lines from a cross between Pasto $x$ Red Carina (hereafter referred to as PxRC).

\subsection{Experimental conditions}

The experiment was conducted under a shelter with open sides at the experimental site Nergena $\left(51^{\circ} 59^{\prime} 46.4 " \mathrm{~N} 5^{\circ} 39^{\prime} 29.4 " \mathrm{E}\right.$, Bennekom) of Unifarm, Wageningen University \& Research from April- August 2018. Light transmission of the transparent cover was $80 \%$. Seeds were sown in potting soil and transplanted to polythene bags filled with cocopeat two weeks after sowing. Each bag contained 10 plants and two bags together were considered one experimental unit (20 plants). The plant density was 54 plants $/ \mathrm{m}^{2}$, which is the density that produces a maximal crop yield in the field. The polythene bags were placed on gutters to facilitate drainage. The experiment consisted of 360 experimental plots and the trial was surrounded by 120 extra plots to avoid border effects. The two mapping populations (40 AxRC F2 lines and 80 PxRC F2 lines) were planted without replicates in two treatments (control (without extra salt) and a salt treatment of $250 \mathrm{mM} \mathrm{NaCl}$ ). In addition, ten replicates per treatment of the six commercial varieties (Atlas, Pasto, Red Carina, Jessie, Dutchess and Titicaca) were included. The plants were irrigated with half-concentrated Hoagland's nutrient solution. Salt treatment started five weeks after sowing ( 3 weeks after transplanting). Salt was applied by incremental increases of $75 \mathrm{mM} \mathrm{NaCl}$ per day until the desired salt concentration was reached, and the soil salt concentration was monitored continuously by measuring the electrical conductivity in the drainage collected from the gutters with a conductivity meter (Profline Cond 315i, Xylem Analytics, Germany). Irradiance, air temperature, water 
content and Electrical Conductivity (EC) in the pots was monitored via wireless sensors (Flower Power ${ }^{\mathrm{TM}}$ ).

\subsection{Assessment of morphological and physiological traits}

Plants were harvested at maturity; plants growing under stress conditions were harvested 135 days after sowing (DAS) and plants growing under control conditions 145 DAS. Seed yield and biomass of stems were measured per plot and reported as $\mathrm{g}$ per $\mathrm{m}^{2}$. Thousand seed weight (TSW) was recorded using a seed counter (Contador, Pfeuffer $\mathrm{GmbH}$, Jefferson, OR, USA) and harvest index (HI) was calculated as the ratio of dry seed weight to dry stem biomass per plot. The average plant height per plot was measured in $\mathrm{cm}$. The salt tolerance index (STI) was calculated as the ratio of seed yield of salt-treated plants and the seed yield of control plants. The developmental stage of the plants was monitored every three days and the flowering time was defined as the number of DAS at which the glomeruli of at least $50 \%$ of the plants in a plot showed anthers.

Several physiological traits were measured at the average onset of flowering (70 DAS), 5 weeks after the start of the salt treatment: stomatal conductance ( $g s)$, relative water content (RWC), specific leaf area (SLA) and ion contents in leaves. Stomatal conductance $(g s)$ was measured on the abaxial side of the second fully developed non-shadowed leaf using a portable leaf porometer (Decagon Devices Inc., WA, Australia) between 9:00 and 15:00 hours on a sunny day. Relative water

content was calculated as $R W C=\frac{(F W-D W)}{(T W-D W)} * 100 \%$, where $\mathrm{FW}$ is the fresh weight, DW is the dry weight and TW is the turgid weight of an entire single young leaf. Turgid weight was determined after the leaf was imbibed in ultrapure water (Milli$Q \circledast$ ) in the dark for 12 hours. The RWC reported per plot was calculated as the average measurement of three young leaves (from three different plants). From the same young leaves, the specific leaf area (SLA) was calculated as the leaf area per unit dry weight $\left(\mathrm{cm}^{2} / \mathrm{g}\right)$.

lon contents in young leaves were measured 5 and 11 weeks after the start of the salt treatment for all the plots and two more measurements were done at 2 and 8 weeks after the start of the salt treatment for the commercial varieties. Ion contents were measured using lon Chromatography as described before (Jaramillo Roman et al. 2020). Briefly, $25 \mathrm{mg}$ of dry sample were turn into ashes in a furnace at $550{ }^{\circ} \mathrm{C}$ for $5 \mathrm{~h}$. Ashes were dissolved in $1 \mathrm{~mL} 3 \mathrm{M}$ formic acid at constant shaking of $500 \mathrm{rpm}$ for $15 \mathrm{~min}$ at $99^{\circ} \mathrm{C}$. The dissolved ashes were $400 \mathrm{x}$ diluted with Milli$Q \circledast$ water and injected on the IC column. Ion contents were calculated as the amount of ions per unit of dry weight ( $\mathrm{mg}$ ion/ $\mathrm{g}$ dry mass) and the ion concentrations were 
estimated considering the water content of the tissue based on the difference between fresh and dry weights.

\subsection{Genotyping of mapping populations and genetic linkage map construction}

A genetic DNA marker map was previously constructed for the F2 population AxRC. The DNA markers are bi-allelic "context" markers, using read counts of the phased alleles of the parents on a consecutive stretch on the assembly of up to 500 SNVs (usually SNPs, but INDELS are also used) (Jarvis et al. 2017).

For the PxRC population, total genomic DNA was extracted from $30 \mathrm{mg}$ of freeze-dried leaf tissue, collected from a mixture of $20 \mathrm{~F} 3$ plantlets per genotype using a CTAB based protocol as previously described (Doyle 1991). The quality of the extracted DNA from the 80 PxRC lines was evaluated with agarose gel electrophoresis and quantified using NanoDrop ${ }^{\mathrm{TM}}$ One (Thermo Scientific, Madison, USA) and Qubit ${ }^{\mathrm{TM}}$ Fluorometric Quantitation (Invitrogen, Carlsbad, USA).

The genotyping of the mapping population PxRC was performed using Single primer enrichment technology (SPET) (Tecan Genomics, San Carlos, CA, USA). Genome assemblies and sequence data from Chenopodium quinoa deposited at NCBI under the BioProject code PRJNA306026 (Jarvis et al. 2017) and the polymorphisms found from $40 \mathrm{X}$ resequencing of Atlas and Red Carina were used to define a panel of 6357 unique SNP targets. For each potential target SNP position, two SPET primers were designed of $40 \mathrm{nt}$ that were unique in the genome using the customized probe design by Tecan Genomics (San Carlos, USA). An overview of the main steps of the SPET is shown in Figure 1. The sequencing of the libraries was performed with Illumina MiSeq ${ }^{\mathrm{TM}}$ (2x300 bp read lengths) (Illumina Inc., San Diego, CA, USA). The average read size was $430 \mathrm{bp}$, and the adaptor sequences were removed using Trimmomatic (Bolger et al. 2014). Sequencing reads were mapped to the reference quinoa genome (Jarvis et al. 2017) using the BurrowsWheeler Aligner (BWMA-MEM algorithm) (Li and Durbin 2009). SNPs were called in the parents from a file containing the probe target sequences of the 80 PxRC lines merged using the SAMtools software package (Li 2011; Li et al. 2009). SNPs were selected when homozygous and polymorphic in the parents (allele 1 frequency $>95$ $\%$ and $<5 \%$ for allele 2) and when the hypothetical heterozygous F1, constructed by merging all mapped reads of the 80 F2-genotypes, had allelic frequency between 20 and $80 \%$. Allele frequencies were calculated per genotype and genotype marker scores were assigned. An allele frequency $>0.9$ was assigned as a (homozygous Pasto), <0.1 as $\boldsymbol{b}$ (homozygous Red Carina), and in between as $\boldsymbol{h}$ (heterozygous). 
A genetic map of the PxRC population was constructed using regression mapping and the Kosambi's mapping function of JoinMap ${ }^{\circledR} 5.0$ (Kyazma, Wageningen, The Netherlands). Regression mapping was performed using a recombination frequency of less than 0.49 and a LOD value larger than 1.0. The chisquare goodness of fit threshold $\left(X^{2}\right)$ was set at 12. A total of 1359 SNPs distributed over 227 scaffolds (from the PacBio/Bionano/Dovetail assembly in Jarvis et al. 2017) were identified for the PxRC population, from which 1231 markers were mapped into 32 linkage groups. Finally, a novel joined map combining the maps of the AxRC and PxRC populations was constructed using JoinMap ${ }^{2} .0$ (Kyazma, Wageningen, The Netherlands). For this purpose, anchor markers were selected (markers present on both maps and found in the same scaffold and maximally $200 \mathrm{kbp}$ apart on the reference genome). The novel combined map contained 17 linkage groups (while 18 chromosomes are present) and some residual parts of the two maps that could not be combined, and a total of 2029 SNPs. 


\section{DNA enzymatic fragmentation}

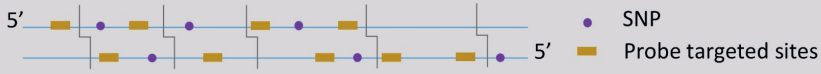

Fragmentation program: $25^{\circ} \mathrm{C}: 15 \mathrm{~min} \rightarrow 70^{\circ} \mathrm{C}: 10 \mathrm{~min}$

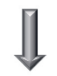

\section{Indexed adaptors ligation}
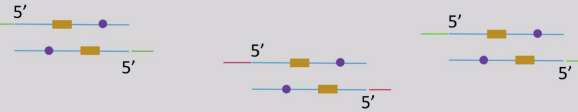

Ligation program: $25^{\circ} \mathrm{C}: 30 \mathrm{~min} \rightarrow 70^{\circ} \mathrm{C}: 10 \mathrm{~min}$

\section{$\prod$}

3. Probes hybridization, enrichment and purification
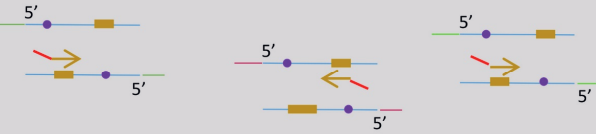

Hybridization and extension program: $95^{\circ} \mathrm{C}: 5 \mathrm{~min} \rightarrow 200$ cycles $\left(80^{\circ} \mathrm{C}-10 \mathrm{~s}\right.$, decrease $0.1^{\circ} \mathrm{C}$ each cycle) $\rightarrow 60^{\circ} \mathrm{C}: 12 \mathrm{~h} \rightarrow 72^{\circ} \mathrm{C}: 10 \mathrm{~min}$

\section{$\mathbb{1}$}

\section{Library amplification}

$$
\begin{aligned}
& \mathrm{REV} \underset{5^{\prime}}{\rightarrow}=\leq \mathrm{FWD} \\
& \mathrm{FWD} \geq=\quad 5^{\prime}<\mathrm{REV}
\end{aligned}
$$

Amplification program: $95^{\circ} \mathrm{C}: 3 \mathrm{~min} \rightarrow 22$ cycles $\left(95^{\circ} \mathrm{C}-30 \mathrm{~s}, 62^{\circ} \mathrm{C}-15 \mathrm{~s}, 72^{\circ} \mathrm{C}-20 \mathrm{~s}\right) \rightarrow 72^{\circ} \mathrm{C}$ : $2 \mathrm{~min}$

\section{$\llbracket$}

\section{Sequencing \\ Nugene \\ Custom primer \\ $\rightarrow$ Illumina Index Primer \\ Probe SNP Library Insert Indexed \\ adaptor}

Miseq

Figure 1. Schematic representation of the Single Primer Enrichment Technology (SPET). 


\subsection{QTL mapping}

Quantitative trait locus analysis was performed with MapQTL® 6 (Kyazma, Wageningen, The Netherlands) using the Restricted MQM mapping function. The threshold for the detection of a QTL was a LOD value of 4.0 , which was determined based on a test of 10000 permutations (Van Ooijen 2009). Cofactors were added in the vicinity of the QTL to increase the power of detection of QTLs. To avoid overfitting, the percentage variance explained by each QTL model was not higher than the $h^{2}$ of the corresponding trait in the respective population. One-LOD and twoLOD support intervals were determined to indicate the uncertainty of the position of the QTL. The localization of the QTLs in the genetic maps were depicted using MapChart@ (Voorrips 2002).

\subsection{Statistical analysis}

The trial was performed following a standard augmented design. The mapping populations were sown in non-replicated plots, while the commercial varieties were sown in ten replicated plots per treatment. Equal variances were assumed for the whole trial, so the residual variance from the varieties was used as an estimate of the residual variance of the populations. General analyses of variance (ANOVA) were performed to determine the significance of genotypic differences, salt treatment differences and their interactions $(p<0.05)$. Narrow sense heritability $\left(h^{2}\right)$ was calculated as: $h^{2}=\frac{\sigma_{G}{ }^{2}}{\sigma_{P}{ }^{2}}=\frac{\sigma_{P}{ }^{2}-\sigma_{E}{ }^{2}}{\sigma_{P}{ }^{2}} \times 100 \%$, where $\sigma_{G}{ }^{2}$ is the genetic variance, $\sigma_{P}{ }^{2}$ is the total phenotypic variance in each mapping population and $\sigma_{E}{ }^{2}$ is the residual variance determined from the ANOVA for each trait. Multiple comparison analyses were performed using Fisher's protected least significant difference (LSD) test on varietal means. Inter-relationships between agronomical and physiological traits were analysed by means of Pearson correlations. All statistical analyses were performed using the software Genstat 19th Edition (VSN International Hemel Hempstead, UK). Principal component analysis (PCA) was used as a multivariate analysis tool to understand the interrelationship between genotypes and traits. PCAs were performed using the prcomp() function in $R(R 2019)$. 


\section{Results}

\subsection{Evaluation of salt tolerance traits in quinoa commercial varieties under field-grown conditions}

Six European quinoa commercial varieties were evaluated for agronomical and salt tolerance-related traits in field-grown conditions under a salt treatment of $250 \mathrm{mM} \mathrm{NaCl}$. The commercial varieties evaluated in this study displayed high diversity for seed yield (Figure 2A). Two main groups could be distinguished. Atlas, Dutchess and Red Carina were the tallest varieties in the experiment (Figure 2B), with an average high seed yield of $614 \mathrm{~g} / \mathrm{m}^{2}$; and Jessie, Pasto, Titicaca formed a second group of shorter varieties with a lower seed yield (average $347 \mathrm{~g} / \mathrm{m}^{2}$ ). The seed yield of Pasto and Titicaca was hardly affected by the $250 \mathrm{mM} \mathrm{NaCl}$ salt treatment. The yields of the other varieties were more affected by salt, with the highest salinity-induced seed yield reduction of $39 \%$ for Jessie (Figure 2A). The height of all the varieties was significantly reduced by salt, by on average $30 \%$ (Figure 2B). The least affected agronomical trait evaluated in this study was the harvest index that was reduced only on average by $7 \%$ under saline conditions, with no significant differences between treatments for Pasto and Titicaca (Figure 2C). The thousand seed weight (TSW) was also hardly affected by salinity; the mostaffected variety was Jessie (33\% reduction), while no salt effect was observed in the TSW of Red Carina and Titicaca (Figure 2D). The phenological development of the plants was monitored throughout the season; flowering time was only significantly delayed in the variety Atlas and not affected in the other varieties (Figure $3 A)$.

Several physiological traits that may contribute to salt tolerance were evaluated to better understand the responses of quinoa to salt stress and the variation between varieties. The stomatal conductance ( $g s)$, measured after five weeks of salt treatment, was reduced by on average $30 \%$ without significant differences between varieties (Figure 3B). Leaf relative water content (RWC) was also significantly reduced in all the varieties, with the lowest reduction observed in the most tolerant varieties Pasto (8\% reduction) and Titicaca (10\% reduction) (Figure 3C). The specific leaf area (SLA) was increased by salinity in Atlas, Jessie and Red Carina, but not significantly affected by salt in Dutchess, Pasto and Titicaca (Figure 3D). The chlorophyll content in leaves was similar for most of the varieties in both treatments, except for Pasto in which it was significantly increased by salt, and Jessie, in which it was significantly reduced (Figure 3E). The maximum quantum 
yield of PSII (Fv/Fm), a measure of the initial light use efficiency of leaves, was not affected by the salt treatment (Figure $3 \mathrm{~F}$ ).

\section{$\square$ OmM_NaCl $\square 250 \mathrm{mM} \_\mathrm{NaCl}$}
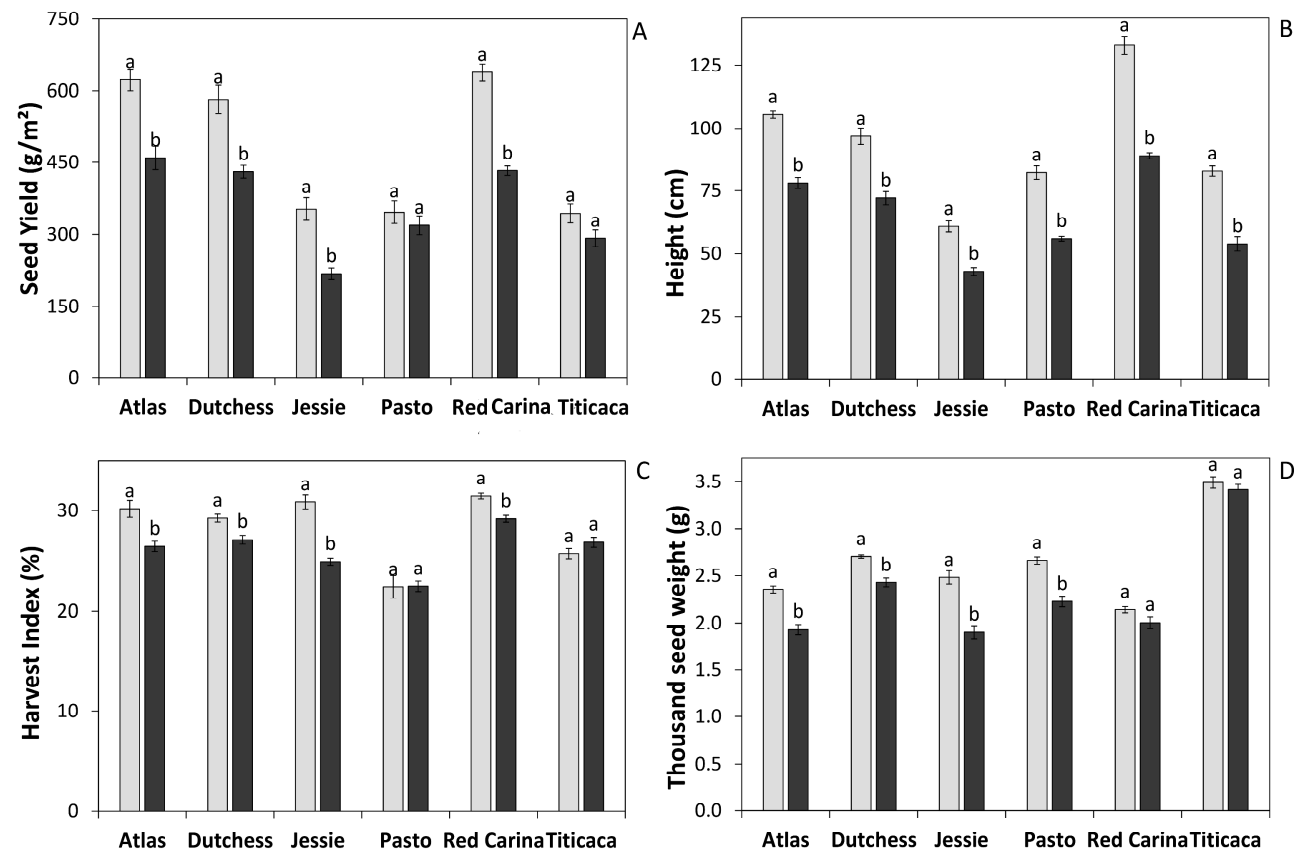

Figure 2. Agronomical traits of six commercial quinoa varieties growing at 0 and $250 \mathrm{mM} \mathrm{NaCl}$. A) Seed yield. B) Plant height. C) Harvest Index. D) Thousand seed weight. Means of 10 plots. Error bars indicate SE of individual means. Statistically significant differences $(p \leq 0.05)$ between treatments (for each variety) are shown with different letters. 
$\square$ OmM_NaCl $\square 250 \mathrm{mM} \_\mathrm{NaCl}$
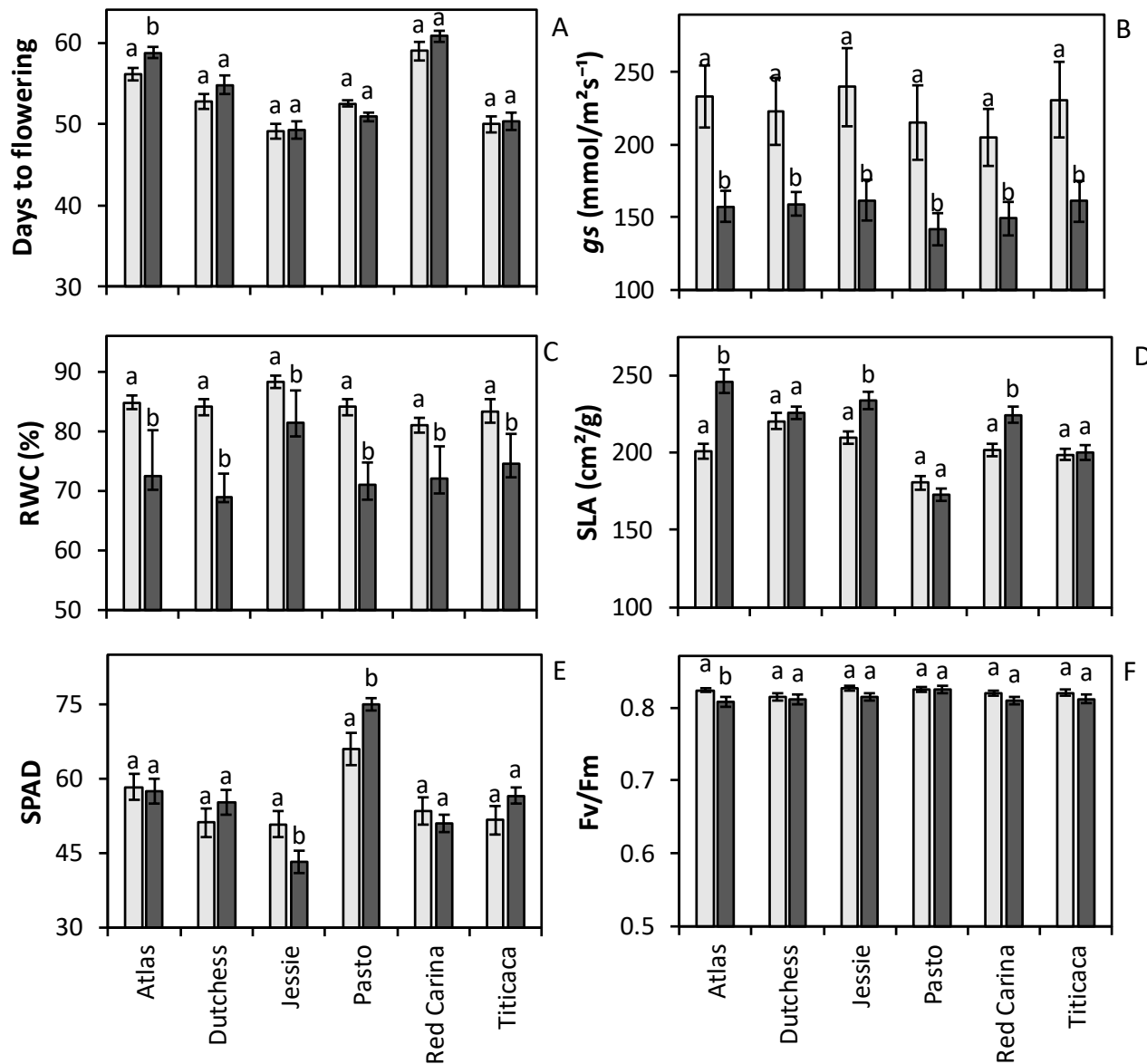

Figure 3. Physiological traits of six commercial quinoa varieties growing at 0 and $250 \mathrm{mM} \mathrm{NaCl}$ measured ten weeks after sowing (five weeks after the start of the salt treatment). A) Days to flowering. B) Stomatal conductance (gs). C) Relative water content of leaves (RWC). D) Specific leaf area (SLA). E) SPAD. F) $\mathrm{Fv} / \mathrm{Fm}$. Means of 10 plots. Error bars indicate SE of individual means. Statistically significant differences $(p \leq 0.05)$ between treatments (for each variety) are shown with different letters. 
Ion contents in young leaves were monitored throughout the growing season (Figure 4). Two weeks after the application of salt, $\mathrm{Na}^{+}$content in the leaves was increased in the salt-stressed plants compared to the controls but after five weeks of salinity it decreased compared to the first time point to $70 \mathrm{mM}$ on average, considerably lower than the level of $\mathrm{Na}^{+}$in the root medium $(250 \mathrm{mM})$. At this time point, which corresponds to the onset of flowering of the plants, $\left[\mathrm{Na}^{+}\right]$was only significantly higher under salt conditions compared to control in the leaves of Jessie and Titicaca, pointing to strong $\mathrm{Na}^{+}$exclusion. Eight weeks after stress, however, the average $\left[\mathrm{Na}^{+}\right]$in young leaves had strongly increased to $390 \mathrm{mM}$ on average, considerably higher than the concentration in the root medium. The last measuring time was 11 weeks after the start of the stress where $\left[\mathrm{Na}^{+}\right]$was higher than at the previous time point for Atlas, Dutchess and Red Carina; but not for Jessie, Pasto and Titicaca. Significant differences were found in the $\left[\mathrm{Na}^{+}\right]$between varieties at all time points, especially for Pasto. This variety had the lowest $\left[\mathrm{Na}^{+}\right]$throughout the whole season; during flowering time the concentration of $\mathrm{Na}^{+}$was of $47 \mathrm{mM}$, compared to a concentration of $41 \mathrm{mM}$ under control conditions. The highest concentration measured for Pasto was $230 \mathrm{mM}$, eight weeks after the start of the salt treatment, still lower than the $\left[\mathrm{Na}^{+}\right]$in the root medium. The concentration of $\mathrm{K}^{+}$ in young leaves was not significantly different between salt-treated and control plants in most of the varieties and measured time points. The general pattern observed for this ion was an increase in its concentration through the time until the last time point (close to maturity) when the concentration dropped, possibly due to a re-localization of assimilates and $\mathrm{K}^{+}$towards seed filling.

$\left[\mathrm{Cl}^{-}\right]$in young leaves was significantly higher in stressed plants compared to the controls. Two weeks after the start of the treatment, the average $\left[\mathrm{Cl}^{-}\right]$was 220 $\mathrm{mM}$. The lowest concentration in the season was also measured at the onset of flowering, but it was still considerably higher than the controls, and higher than $\left[\mathrm{Na}^{+}\right]$. At the last measured timepoint (11 weeks after the start of the stress) the $\mathrm{Cl}^{-}$content was lower than at eight weeks after stress in all the varieties, except Dutchess.

The $\mathrm{K}^{+} \mathrm{Na}^{+}$ratio in young leaves was reduced by salinity in most timepoints for most varieties, except for Atlas, Dutchess, Pasto and Red Carina at the onset of flowering (5 weeks after stress), where not significant differences were found between treatments. Pasto had the highest $\mathrm{K}^{+} \mathrm{Na}^{+}$in salt stressed plants throughout the whole growing period. 

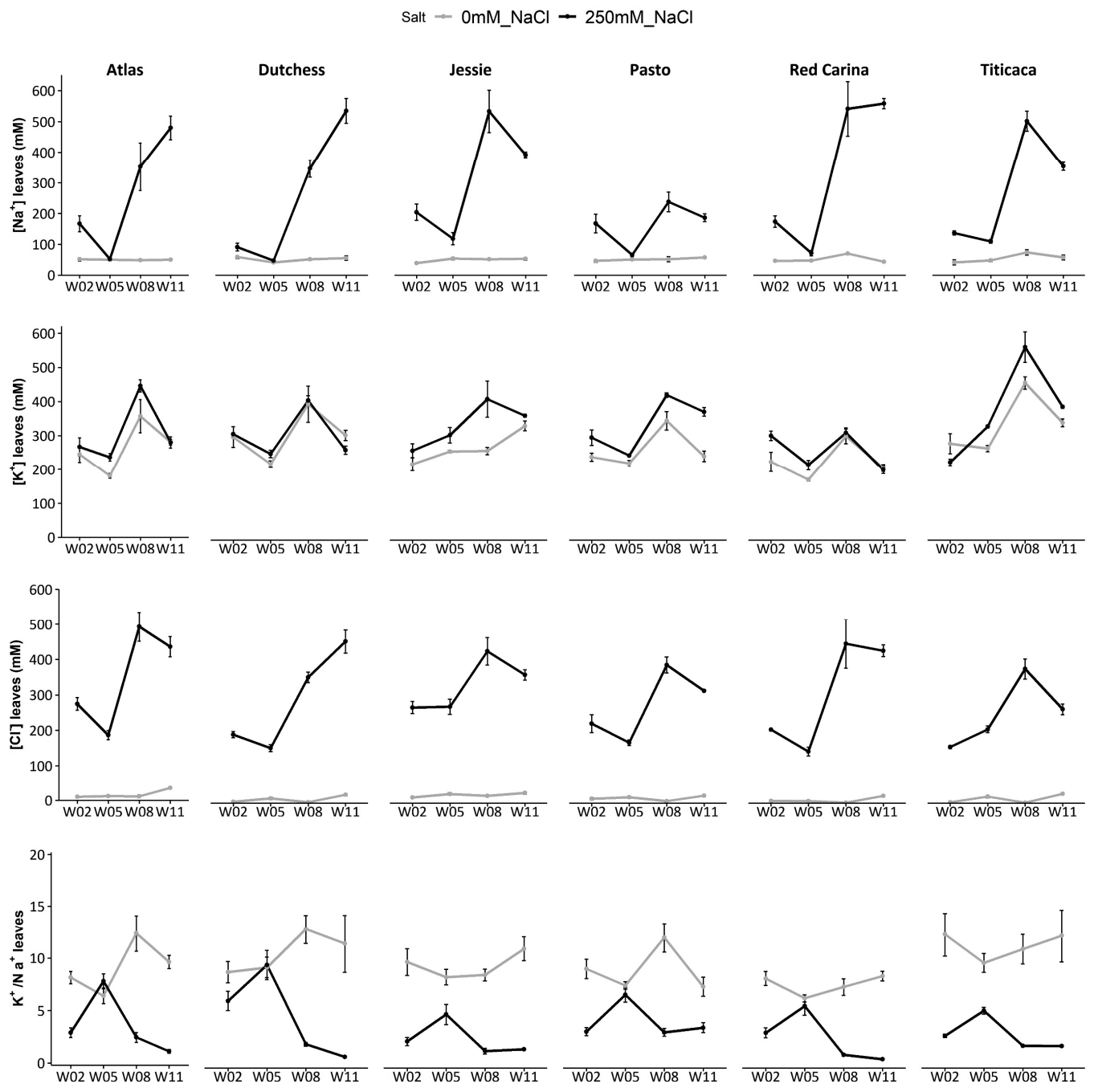

Figure 4. $\mathrm{Na}^{+}, \mathrm{K}^{+}, \mathrm{Cl}^{-}$contents and $\mathrm{K}^{+} / \mathrm{Na}^{+}$ratio in young leaves measured throughout the growing season $(2,5,8$ and 11 weeks after the start of salt treatment) in the six commercial varieties growing at 0 and $250 \mathrm{mM} \mathrm{NaCl}$. Means of 6 plots. Error bars indicate SE of individual means. 


\subsection{Genetic diversity for salt tolerance traits in the mapping populations}

High variation in seed yields was observed for all the evaluated genotypes of the mapping population (Figure 5). Seed yields ranged from 136 to $889 \mathrm{~g} / \mathrm{m}^{2}$ under control conditions and from 120 to $660 \mathrm{~g} / \mathrm{m}^{2}$ under saline conditions (Table 1). Clear differences were observed between the two mapping populations (AxRC and PxRC). Higher yields were measured in genotypes from the AxRC population, while the PxRC population had lower yields but higher salt tolerance. In fact, some PxRC genotypes (above the dotted line in Figure 5) even performed better under salt conditions compared to control.

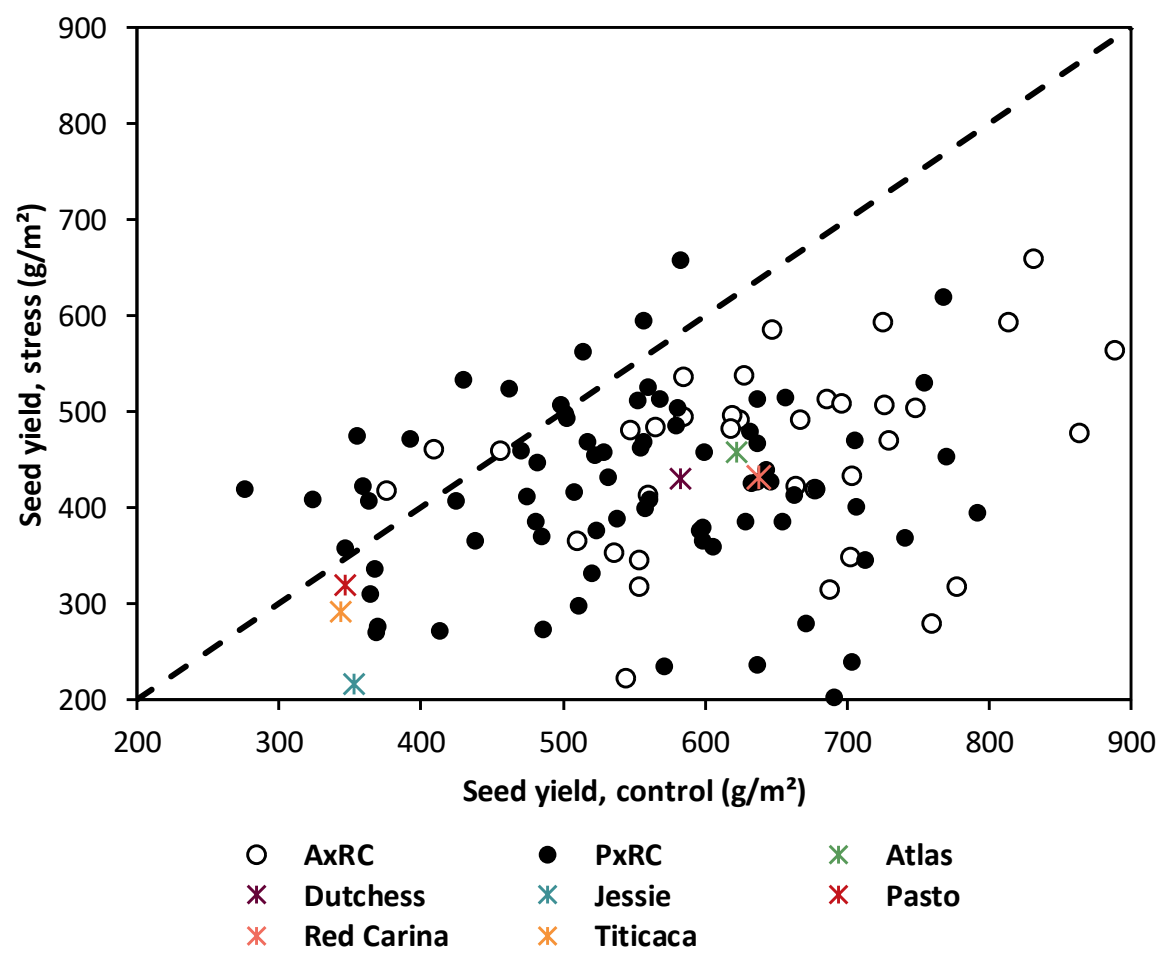

Figure 5. Overall distribution of genotypes based on their seed yields under control $(0 \mathrm{mM} \mathrm{NaCl})$ and stress $(250 \mathrm{mM} \mathrm{NaCl})$ conditions. On the $x$-axis seed yields under control conditions are depicted, and on the $y$-axis the seed yields under stress conditions. Genotypes above the dotted line had a better performance under stress conditions compared to control. 
For both populations, we evaluated several agronomical parameters and traits that may contribute to salt tolerance. The parental mean values, summary statistics for the mapping populations and narrow sense heritability for each trait are presented in Table 1. Frequency distributions are displayed in Figures 6, 7 and 8. For all the analysed traits, the populations followed a continuous distribution representing a normal phenotypic segregation suitable for QTL mapping. For all the traits the segregation was transgressive, meaning that a number of genotypes exceeded the trait parental values.

The frequency distributions for agronomical traits are depicted in Figure 6A$\mathrm{H}$. In general, a wider segregation of most of the agronomical traits was observed in the progeny of PxRC compared to $A x R C$, in line with the more contrasting parental values between Pasto and Red Carina compared to Atlas and Red Carina. Seed yield and plant height of the populations partially overlapped for both treatments, and higher yields and heights were observed for AxRC (Figure 6A-D). Harvest index was the least affected agronomical trait by salinity for both populations; on average $27 \%$ and $25 \%$ of assimilates were relocated into seed under control and stress conditions, respectively (Figure E, F). Thousand seed weight showed a higher variation in the PxRC than the AxRC progeny, but on average, the salt treatment reduced TSW by $20 \%$ in both populations (Figure $6 \mathrm{G}, \mathrm{H}$ ). The impact of salinity on flowering time was more noticeable in the populations compared to the varieties; some genotypes of the PxRC population even needed up to 75 days to start flowering (from an average of 55 days in this population) (Figure 7A, D). The specific leaf area was not significantly affected by salinity in most of the PxRC genotypes, while in the AxRC population salinity increased SLA (Figure 7B, E). In both populations, relative water content decreased from $80 \%$ on average under control conditions to $70 \%$ on average under salt treatment (Figure 7C, F). Stomatal conductance showed a wide variation in both treatments and populations, but it was reduced by salinity by on average $30 \%$ in AxRC and $40 \%$ in PxRC (Figure 7G, J). Chlorophyll content was slightly increased by salinity in PxRC and hardly affected in AxRC (Figure $7 \mathrm{H}, \mathrm{K}$ ). The maximum quantum yield of PSII (Fv/Fm) was not affected by salinity in both populations (Figure 7I, L).

The ion contents in young leaves were measured in both mapping populations at two time points: at the onset of flowering (5 weeks after the start of salt stress) and towards maturity (11 weeks after the start of the treatment) (Figure $8 \mathrm{~A}-\mathrm{P})$. At the first timepoint, $\left[\mathrm{Na}^{+}\right]$in leaves was just slightly increased by salinity from on average $40 \mathrm{mM}$ under control to $55 \mathrm{mM}$ under stress. However, 11 weeks after salinity, $\left[\mathrm{Na}^{+}\right]$in all the genotypes was considerably higher in stressed plants 
compared to controls and higher in the AxRC population (average $531 \mathrm{mM}$ ) compared to PxRC (average $390 \mathrm{mM}$ ) (Figure 8A-D). Five weeks after stress, [K+] in young leaves was higher under stress conditions compared to control, while its concentration was not significantly affected by salinity after 11 weeks of stress. Differences between populations were clearer at the second timepoint than at the first one; the average $\left[\mathrm{K}^{+}\right]$was higher in PxRC (260 mM) than in AxRC (220 mM) (Figure 8E-H). No significant differences were found at flowering time for the $\mathrm{K}^{+} / \mathrm{Na}^{+}$ ratios between treatments for both mapping populations (Figure 8I-J). However, after 11 weeks of stress $\mathrm{K}^{+} / \mathrm{Na}^{+}$under salinity was significantly lower than under control conditions (Figure $8 \mathrm{~K}-\mathrm{L}$ ). The leaf $\left[\mathrm{Cl}^{-}\right]$was already significantly increased after 5 weeks of salt treatment in stressed plants of both populations (157 mM on average) (Figure 8M-N). Towards maturity, $\left[\mathrm{Cl}^{-}\right]$reached on average $491 \mathrm{mM}$ in AxRC and $386 \mathrm{mM}$ in PxRC (Figure 8O, P).

Narrow sense heritability estimates $\left(h^{2}\right)$ for all the measured traits in both populations and salt concentrations are presented in Table 1. In general, heritabilities for the progeny of PxRC were higher than for AxRC, which is likely caused by the larger size of the first population. The $h^{2}$ for agronomical traits were high, especially for PxRC $\left(63 \%<h^{2}<89 \%\right)$. In contrast, physiological traits had lower $h^{2}$, and for some traits, such as chlorophyll content, gs and Fv/Fm, it was not possible to estimate a genetic component, possibly due to the limited genetic variation of these traits within the populations and, particularly for gs, due to the sensitivity of this measurement to environmental variation. While the $h^{2}$ for the ion contents at the onset of flowering were low, higher $h^{2}$ were found for ion contents at maturity, particularly for the progeny of PxRC under salinity $\left(53 \%<h^{2}<71 \%\right)$. 
Table 1. Parental mean values, summary statistics and narrow-sense heritability for all the traits measured in the parents and mapping populations growing at 0 and $250 \mathrm{mM} \mathrm{NaCl}$.

\section{Control (0 mM NaCl)}

\begin{tabular}{|c|c|c|c|c|c|c|c|c|c|c|}
\hline \multirow[b]{2}{*}{ Trait } & \multirow[b]{2}{*}{ Abbreviation } & \multicolumn{5}{|c|}{ Mean } & \multicolumn{2}{|c|}{ Range } & \multicolumn{2}{|c|}{$h^{2}$} \\
\hline & & Atlas & Pasto & $\mathbf{R C}$ & AxRC & PxRC & AxRC & PxRC & AxRC & PxRC \\
\hline Seed yield $\left(\mathrm{g} / \mathrm{m}^{2}\right)$ & Y_C & 622 & 347 & 638 & 657 & 546 & $375-888$ & $136-792$ & 63.6 & 65.5 \\
\hline Harvest Index (\%) & HI_C & 30.2 & 22.4 & 31.5 & 29.7 & 27.3 & $20.6-34.2$ & $7.6-34.0$ & 3.9 & 68.3 \\
\hline Thousand-seed weight (g) & TSW_C & 2.4 & 2.7 & 2.1 & 2.5 & 2.5 & $1.9-2.8$ & $1.7-3.2$ & 36.9 & 78.4 \\
\hline Height $(\mathrm{cm})$ & $\mathrm{H} \_\mathrm{C}$ & 106 & 82.3 & 133 & 117 & 109 & $95-135$ & $75-140$ & 19.0 & 70.1 \\
\hline Days to flowering & F_C & 56.2 & 52.5 & 59.0 & 55.7 & 53.4 & $49.2-60.8$ & $46-65$ & 34.4 & 38.7 \\
\hline Specific leaf area $\left(\mathrm{cm}^{2} / \mathrm{g}\right)$ & SLA_C & 201 & 181 & 202 & 185 & 179 & $144-228$ & $140-225$ & 42.4 & 36.0 \\
\hline Relative water content (\%) & RWC_C & 84.8 & 84.1 & 80.1 & 82.5 & 81.5 & $70.2-93.6$ & $68.2-94.7$ & 43.0 & 39.5 \\
\hline Chlorophyll content & SPAD_C & 58.3 & 65.9 & 53.4 & 53.7 & 59.2 & $41.4-69.4$ & $39.8-78.1$ & 0.0 & 0.0 \\
\hline Stomatal conductance $\left(\mathrm{mmol} / \mathrm{m}^{2} \mathrm{~s}\right)$ & gs_C & 233 & 215 & 205 & 217 & 242 & 99-399 & $106-406$ & 0.0 & 0.0 \\
\hline $\mathrm{Fv} / \mathrm{Fm}$ & FVM_C & 0.82 & 0.83 & 0.82 & 0.82 & 0.82 & $0.77-0.86$ & $0.78-0.85$ & 60.1 & 72.5 \\
\hline$\left[\mathrm{Na}^{+}\right]$leaves $(\mathrm{mM}) \mathrm{W} 5$ & $\mathrm{Na}$ _5_C & 50.0 & 50.6 & 47.5 & 41.6 & 42.6 & 29.3-54.7 & $24.3-61.3$ & 0.0 & 24.1 \\
\hline$\left[\mathrm{K}^{+}\right]$leaves $(\mathrm{mM}) \mathrm{W} 5$ & K_5_C & 181 & 223 & 176 & 178 & 191 & $140-218$ & $125-278$ & 14.6 & 71.3 \\
\hline$\left[\mathrm{Cl}^{-}\right]$leaves (mM) W5 & Cl_5_C & 12.1 & 12.6 & 8.0 & 13.4 & 8.9 & $5.4-24.6$ & $0.9-19.5$ & 43.6 & 26.9 \\
\hline $\mathrm{K}^{+} / \mathrm{Na}^{+}$leaves $\mathrm{W} 5$ & $\mathrm{KNa}$ 5__C & 6.4 & 7.5 & 6.3 & 7.5 & 7.9 & $5.0-12.0$ & $4.5-17.6$ & 33.2 & 55.9 \\
\hline$\left[\mathrm{Na}^{+}\right]$leaves $(\mathrm{mM}) \mathrm{W} 11$ & $\mathrm{Na}$ _11_C & 50.3 & 57.4 & 43.2 & 45.5 & 46.9 & $27.8-77.8$ & $17.2-77.7$ & 0.0 & 28.2 \\
\hline$\left[\mathrm{K}^{+}\right]$leaves $(\mathrm{mM}) \mathrm{W} 11$ & K_11_C & 281 & 244 & 210 & 214 & 224 & $121-288$ & $141-371$ & 24.5 & 48.0 \\
\hline$\left[\mathrm{Cl}^{-}\right]$leaves (mM) W11 & Cl_11_C & 35.7 & 21.2 & 20.3 & 27.3 & 18.6 & $12.8-50.4$ & $7.3-38.8$ & 42.7 & 0.0 \\
\hline $\mathrm{K}^{+} / \mathrm{Na}^{+}$leaves $\mathrm{W} 11$ & $\mathrm{KNa} 11 \mathrm{C}$ & 9.6 & 7.4 & 8.4 & 8.3 & 8.5 & $4.8-12.1$ & $4.3-16.4$ & 0.0 & 25.4 \\
\hline
\end{tabular}

\section{Stress (250 $\mathrm{mM} \mathrm{NaCl})$}

\begin{tabular}{|c|c|c|c|c|c|c|c|c|c|c|}
\hline \multirow[b]{2}{*}{ Trait } & \multirow[b]{2}{*}{ Abbreviation } & \multicolumn{5}{|c|}{ Mean } & \multicolumn{2}{|c|}{ Range } & \multicolumn{2}{|c|}{$h^{2}$} \\
\hline & & Atlas & Pasto & RC & AxRC & PxRC & AxRC & PxRC & AxRC & PxRC \\
\hline Seed yield $\left(\mathrm{g} / \mathrm{m}^{2}\right)$ & Y_S & 459 & 319 & 433 & 453 & 407 & $187-660$ & $120-659$ & 69.5 & 70.5 \\
\hline Harvest Index (\%) & HI_S & 26.5 & 22.5 & 29.2 & 25.9 & 25.6 & $17.8-61.5$ & $14.0-34.1$ & 75.9 & 89.5 \\
\hline Thousand-seed weight (g) & TSW_S & 1.9 & 2.2 & 2.0 & 2.0 & 2.1 & $1.4-2.7$ & $1.2-2.7$ & 47.4 & 63.1 \\
\hline Height $(\mathrm{cm})$ & H_S & 78.1 & 56.1 & 89 & 81.4 & 76.7 & $64-98$ & $40-115$ & 30.2 & 77.9 \\
\hline Days to flowering & F_S & 58.8 & 50.9 & 60.8 & 60.3 & 55.6 & $49.2-68.7$ & $45.7-74.6$ & 54.5 & 80.6 \\
\hline Specific leaf area $\left(\mathrm{cm}^{2} / \mathrm{g}\right)$ & SLA_S & 246 & 173 & 224 & 210 & 178 & $160-266$ & $137-226$ & 26.3 & 8.2 \\
\hline Relative water content (\%) & RWC_S & 72.4 & 70.9 & 72.1 & 72.3 & 71.9 & $56.1-92.8$ & $54.9-93.3$ & 0.0 & 7.4 \\
\hline Chlorophyll content & SPAD_S & 57.5 & 75 & 50.9 & 60.2 & 66.4 & $46.7-72.8$ & $39.9-83.3$ & 0.0 & 41.9 \\
\hline Stomatal conductance $\left(\mathrm{mmol} / \mathrm{m}^{2} \mathrm{~s}\right)$ & gs_S & 158 & 142 & 149 & 147 & 148 & $77.9-248$ & $91.4-220$ & 9.9 & 0.0 \\
\hline $\mathrm{Fv} / \mathrm{Fm}$ & FVM_S & 0.81 & 0.82 & 0.81 & 0.81 & 0.82 & $0.76-0.84$ & $0.76-0.86$ & 0.0 & 0.0 \\
\hline$\left[\mathrm{Na}^{+}\right]$leaves $(\mathrm{mM}) \mathrm{W} 5$ & Na_5_S & 52.6 & 65.6 & 71.7 & 57.9 & 66.7 & $37.8-92.5$ & $25.0-111$ & 0.0 & 0.0 \\
\hline$\left[\mathrm{K}^{+}\right]$leaves (mM) W5 & K_5_S & 237 & 247 & 219 & 227 & 214 & $183-288$ & $158-300$ & 0.0 & 29.8 \\
\hline$\left[\mathrm{Cl}^{-}\right]$leaves (mM) W5 & Cl_5_S & 187 & 171 & 146 & 168 & 146 & $102-229$ & $87.7-229$ & 0.0 & 23.3 \\
\hline $\mathrm{K}^{+} / \mathrm{Na}^{+}$leaves W5 & $\mathrm{KNa}$ _5_S & 7.8 & 6.7 & 5.6 & 6.9 & 5.9 & $3.8-12.0$ & $2.9-16.4$ & 0.0 & 31.6 \\
\hline$\left[\mathrm{Na}^{+}\right]$leaves (mM) W11 & $\mathrm{Na}$ 11__S & 480 & 186 & 558 & 531 & 391 & $270-875$ & $84.8-699$ & 63.4 & 71.4 \\
\hline$\left[\mathrm{K}^{+}\right]$leaves $(\mathrm{mM}) \mathrm{W} 11$ & K_11_S & 280 & 373 & 205 & 221 & 263 & $153-323$ & $173-438$ & 44.1 & 60.6 \\
\hline$\left[\mathrm{Cl}^{-}\right]$leaves (mM) W11 & Cl_11_S & 436 & 315 & 427 & 491 & 386 & $275-789$ & $180-627$ & 66.3 & 72.6 \\
\hline $\mathrm{K}^{+} / \mathrm{Na}^{+}$leaves $\mathrm{W} 11$ & $\mathrm{KNa}$ 11_S & 1.1 & 3.6 & 0.6 & 0.7 & 1.3 & $0.3-1.4$ & $0.4-4.5$ & 0.0 & 53.7 \\
\hline
\end{tabular}


A
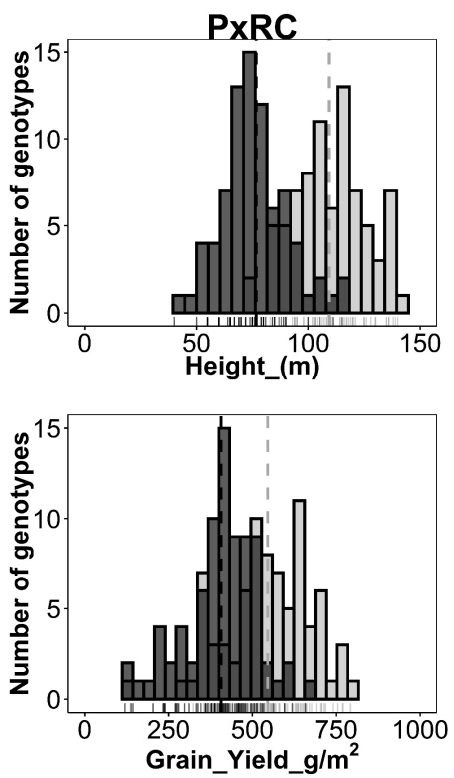

E

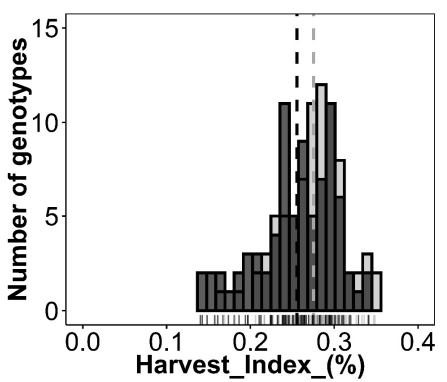

G

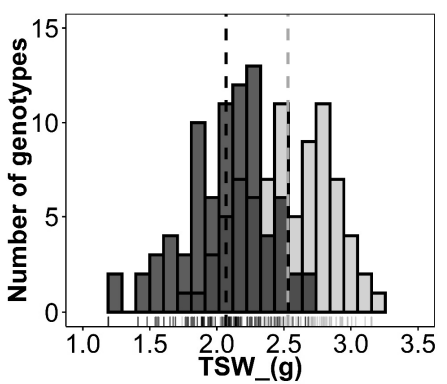

B

$250 \mathrm{mM} \mathrm{NaCl}$

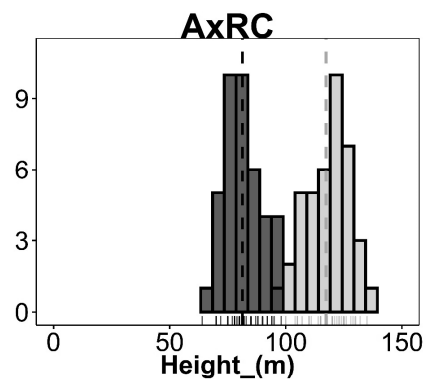

D

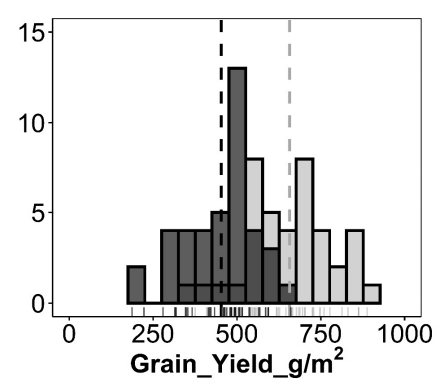

$\mathbf{F}$

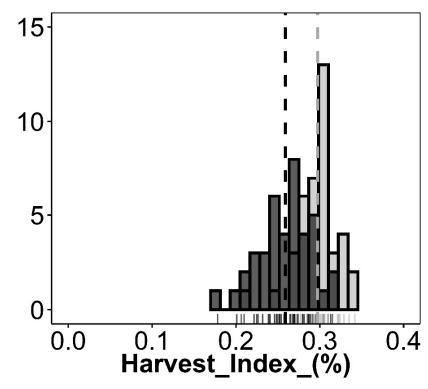

H

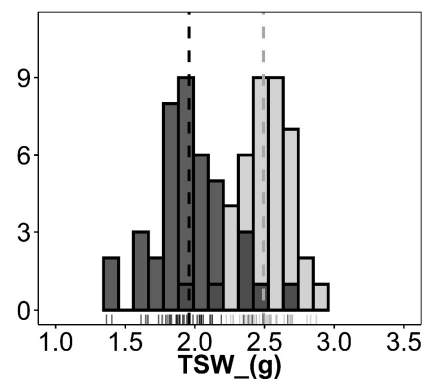

Figure 6. Frequency distributions of genotype values for agronomical traits of both mapping populations growing at 0 and $250 \mathrm{mM} \mathrm{NaCl}$. Units of the $y$-axis denote the number of genotypes. Dotted lines represent the average values of the populations in both treatments. A) Plant height population PxRC. B) Plant height population AxRC. C) Seed yield PxRC. D) Seed yield AxRC. E) Harvest Index PxRC. F) Harvest Index AxRC. G) Thousand seed weight PxRC. H) Thousand seed weight AxRC. 


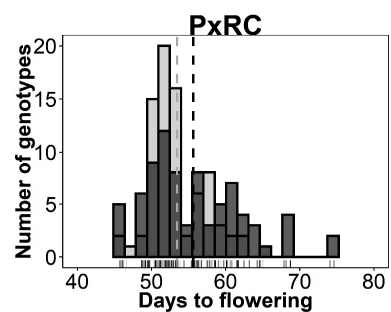

D
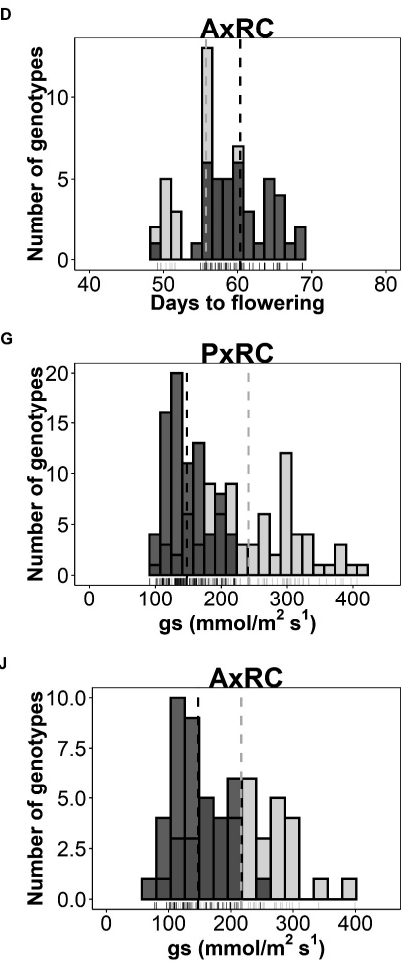

Salt $\square 0 \mathrm{mM} \mathrm{NaCl}$ 回 $250 \mathrm{mM} \mathrm{NaCl}$

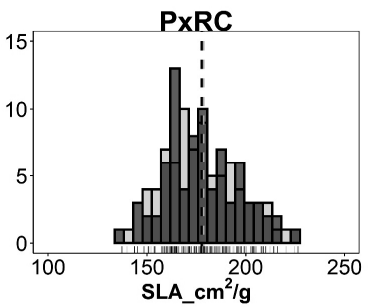

E

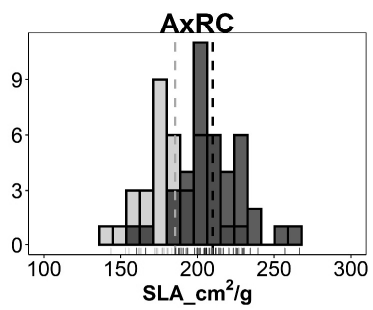

H

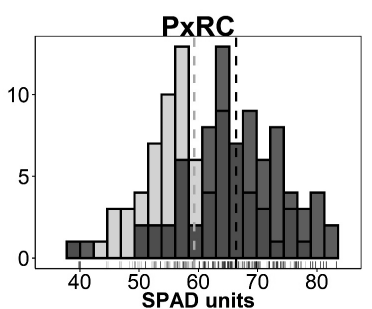

K

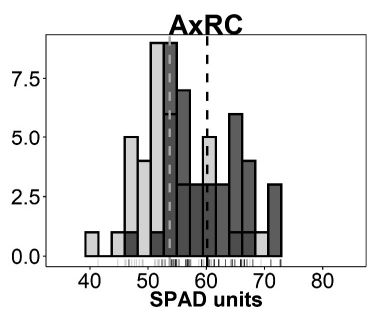

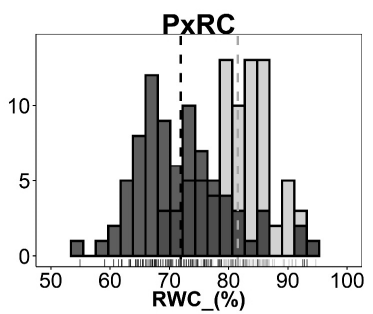

$\mathbf{F}$
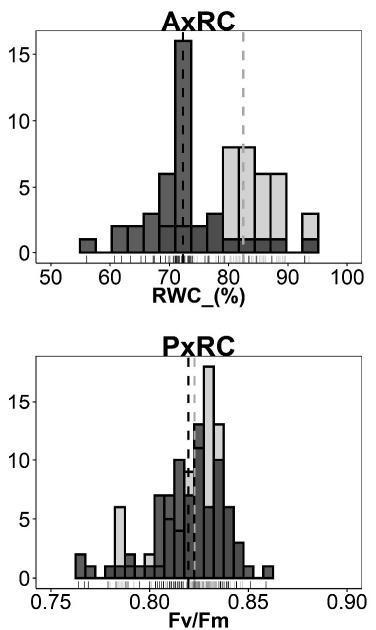

L

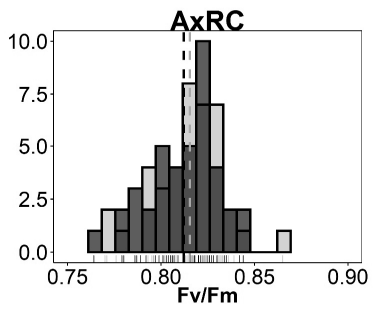

Figure 7. Frequency distributions of genotype values for physiological traits of both mapping populations growing at 0 and $250 \mathrm{mM} \mathrm{NaCl}$. Units of the $y$-axis denote the number of genotypes. Dotted lines represent the average values of the populations in both treatments. A) Flowering time population PxRC. B) Specific leaf area PxRC. C) Relative water content PxRC. D) Flowering time AxRC. E) Specific leaf area AxRC. F) Relative water time AxRC. G) Stomatal conductance PxRC. H) Chlorophyll content PxRC. I) Fv/Fm PxRC. J) Stomatal conductance AxRC. K) Chlorophyll content AxRC. L) Fv/Fm AxRC. 

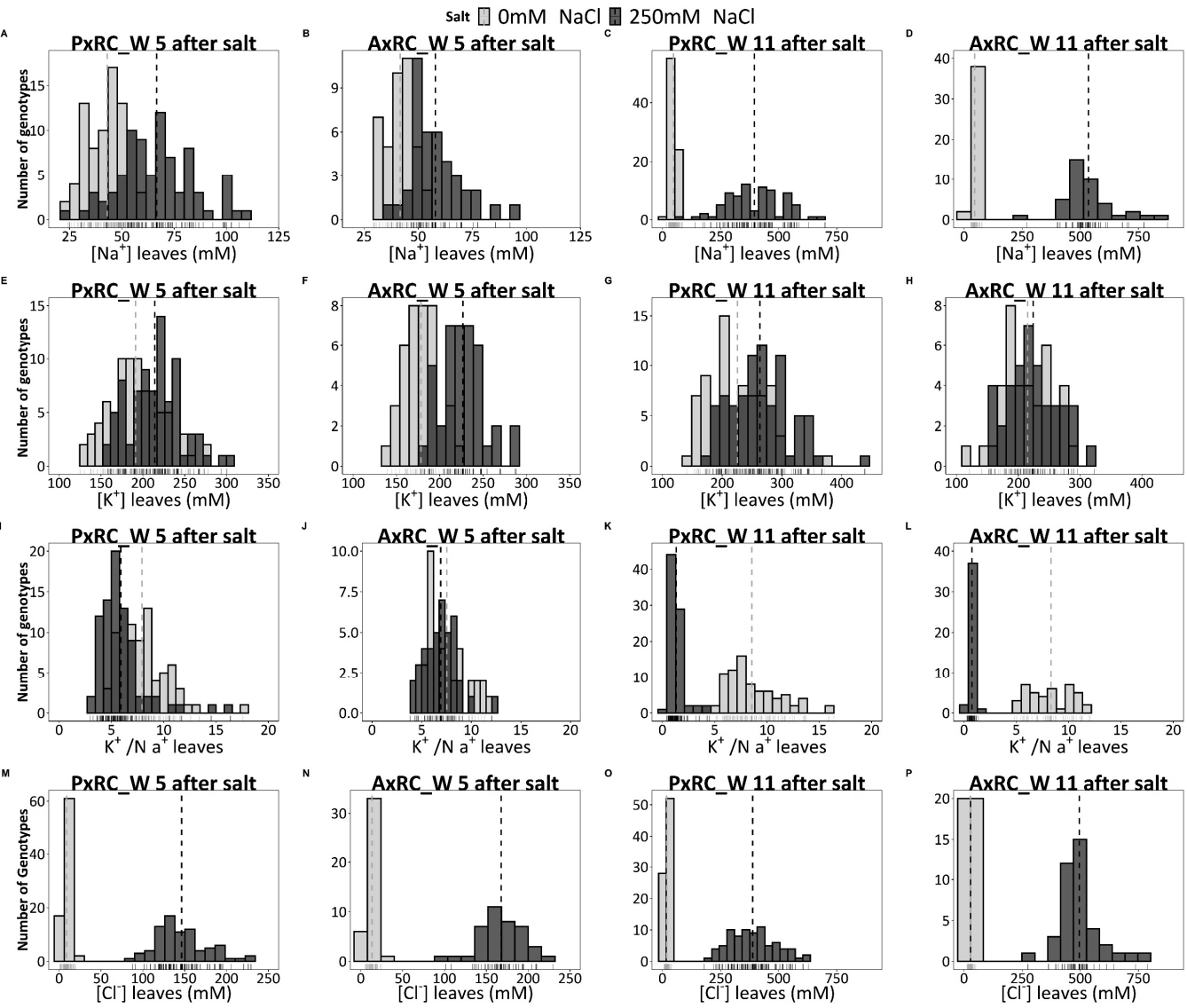

Figure 8. Frequency distributions of genotype values for the ion contents in young leaves of both mapping populations growing at 0 and $250 \mathrm{mM} \mathrm{NaCl}$, measured 10 and 16 weeks after sowing (5 and 11 weeks after the start of salt treatment). Units of the $y$-axis denote the number of genotypes. Dotted lines represent the average values of the populations in both salt treatments. A-D) $\left[\mathrm{Na}^{+}\right]$in leaves. E-H) $\left[\mathrm{K}^{+}\right]$in leaves. I$\mathrm{L}) \mathrm{K}^{+} / \mathrm{Na}^{+}$ratio in leaves. $\left.\mathrm{M}-\mathrm{P}\right)\left[\mathrm{Cl}^{-}\right]$in leaves. 


\subsection{Relationships between traits and salt tolerance in quinoa}

A multivariate analysis (PCA) was done to further characterize genotypic differences for salt tolerance within the populations and to discover the relationships between the analysed traits (Figure 9). The first principal component accounted for $32 \%$ of the total variation and the traits that contributed the most to $\mathrm{PC} 1$ were $\left[\mathrm{Na}^{+}\right]$ and $\left[\mathrm{Cl}^{-}\right]$with an opposite direction to $\mathrm{K}^{+} / \mathrm{Na}^{+}$and $\left[\mathrm{K}^{+}\right]$. The second component, accounting for $15 \%$ of the total variation, resolved genotypes based on seed yield, STI, SLA and SPAD. The PCA distinguished the two mapping populations from each other. The population AxRC was grouped on the left side of the plot with higher $\mathrm{Na}^{+}$ and $\mathrm{Cl}^{-}$contents and a higher SLA; the parents Atlas and Red Carina were included in this group. Pasto, which was positioned on the right side of the biplot pulled the PxRC population more to the right side of the biplot, and these genotypes were grouped with a higher STI, higher chlorophyll content and $\mathrm{K}^{+}$content. The positioning of the parents in the biplot, Atlas and Red Carina on the opposite side of Pasto, might be associated with contrasting salt tolerance strategies between these varieties, and the distinct distribution of the progeny of the populations AxRC and PxRC in the biplot might be explained by the segregation of the traits that relate to these strategies. 


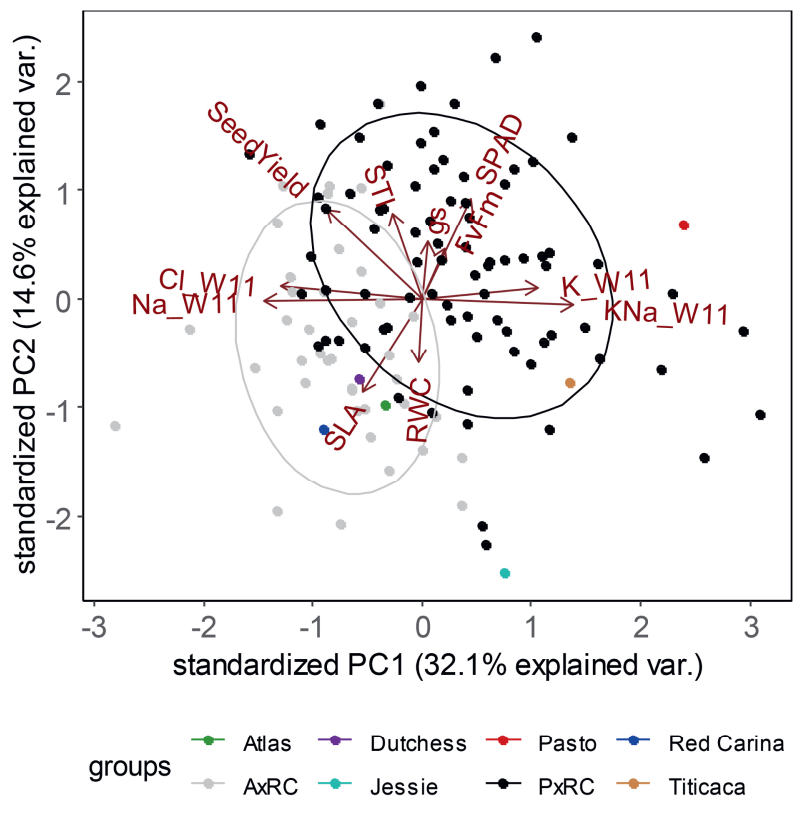

Figure 9. Principal component biplot displaying the variation in performance under stress conditions and salt tolerance sub-traits within the mapping populations and the commercial varieties. Genotypes are indicated as dots and traits as red vectors. 
Correlation analyses were performed to understand the relationships between traits within each mapping population (Figures 10 and 11). For PxRC, the agronomical traits measured under salt stress (seed yield, TSW, HI and STI) had a significant positive correlation with the $\mathrm{Na}^{+}$and $\mathrm{Cl}^{-}$contents and a negative correlation with the $\mathrm{K}^{+} / \mathrm{Na}^{+}$ratio measured at maturity (11 weeks after stress). Flowering time was negatively correlated with the $\mathrm{Na}^{+}, \mathrm{K}^{+}$and $\mathrm{Cl}^{-}$contents. In addition, the SLA had a positive correlation with RWC, $\mathrm{Fv} / \mathrm{Fm}, \mathrm{K}^{+}$and $\mathrm{Cl}^{-}$at the onset of flowering. The ion concentrations at the onset of flowering did not correlate with the ion contents at maturity (Figure 10).

Correlations were different in the progeny of AxRC. The ion contents at maturity were strongly positive correlated with those at the onset of flowering. The SLA was positively correlated with plant height and the $\mathrm{K}^{+} / \mathrm{Na}^{+}$ratio, and negatively correlated with [ $\left.\mathrm{Cl}^{-}\right],\left[\mathrm{Na}^{+}\right]$, gs, RWC and SPAD. For this population, the correlations between agronomical traits and ion contents were weaker: STI and TSW were negatively correlated with $\left[\mathrm{Cl}^{-}\right]$, and seed yield had a negative correlation with $\left[\mathrm{K}^{+}\right]$ and the $\mathrm{K}^{+} / \mathrm{Na}^{+}$ratio (Figure 11 ). 


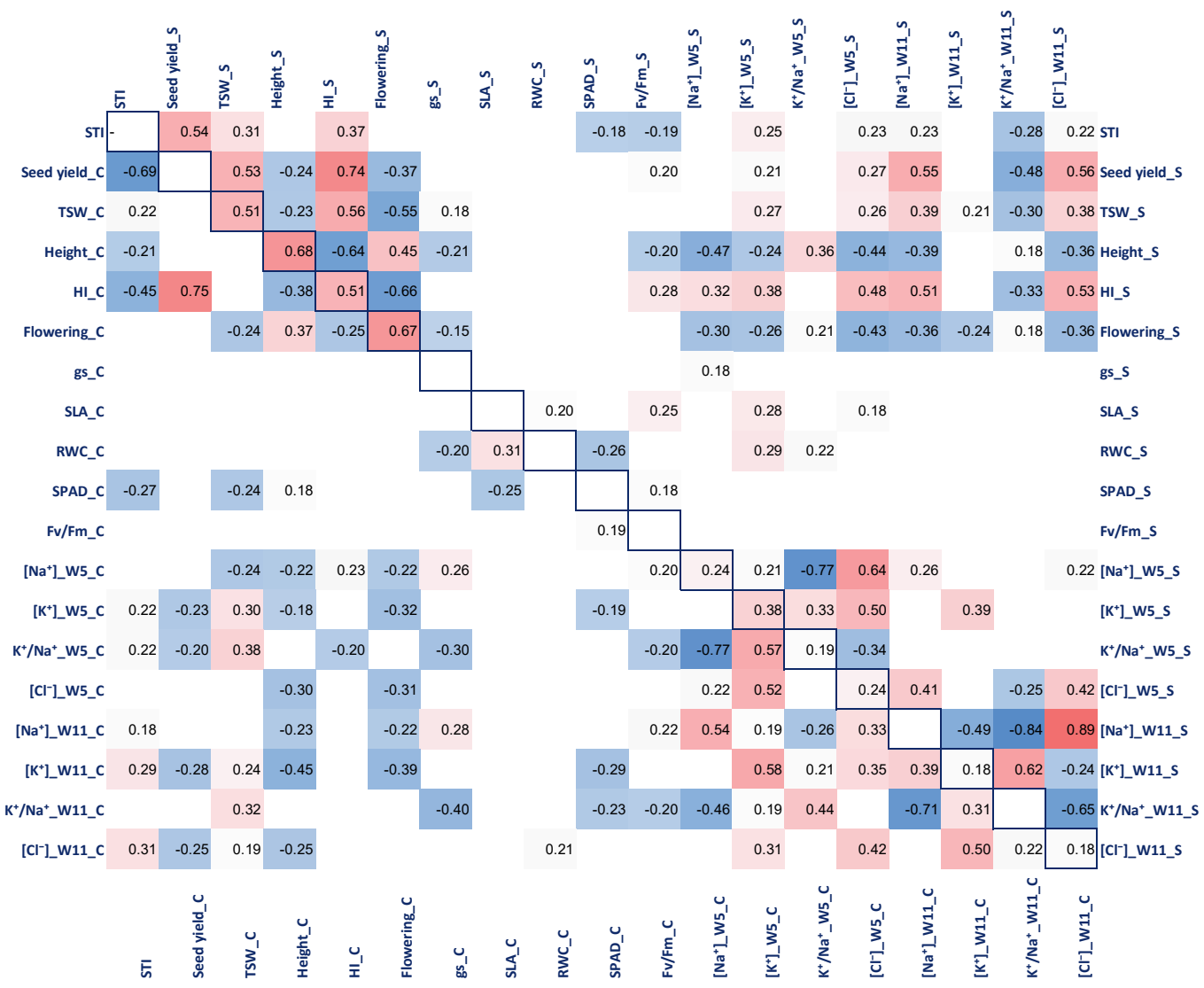

Figure 10. Correlations -of genotypic means- of agronomical and physiological traits in control and stress conditions for the mapping population PxRC. Only Pearson correlation coefficients that differed significantly from zero $(p<0.001)$ are presented. Values in blue indicate a negative correlation and in red a positive correlation between traits. Coefficients in the diagonal indicate the relation of the same trait under control and stress conditions. Below the diagonal the correlations between traits under control conditions are presented and above the diagonal the correlations under stress conditions. 


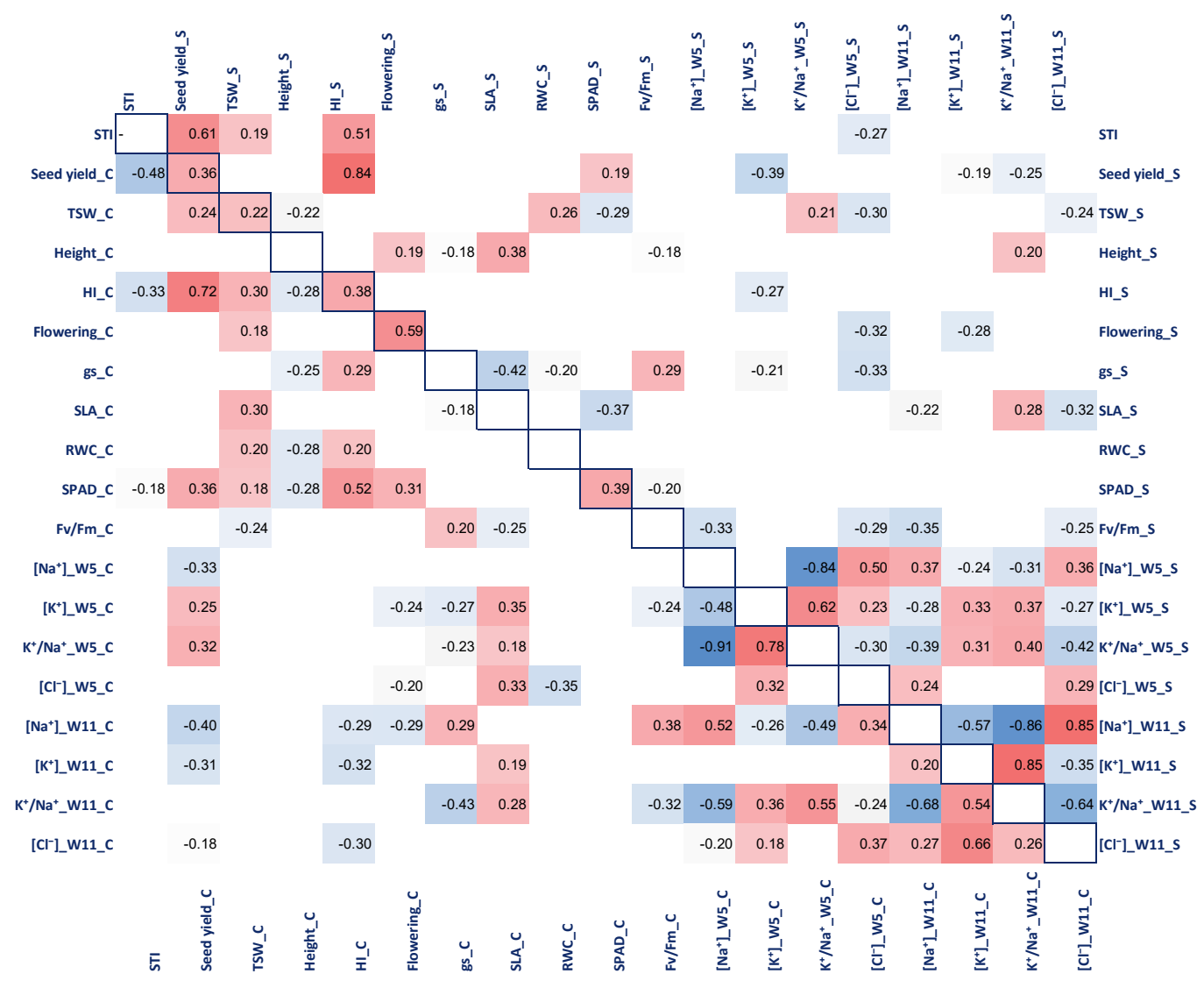

Figure 11. Correlations -of genotypic means- of agronomical and physiological traits under control and stress conditions for the mapping population AxRC. Only Pearson correlation coefficients that differed significantly from zero $(p<0.001)$ are presented. Values in blue indicate a negative correlation and in red a positive correlation between traits. Coefficients in the diagonal indicate the relation of the same trait in control and stress conditions. Below the diagonal the correlations between traits under control conditions are given and above the diagonal the correlations under stress conditions. 


\subsection{Quantitative trait loci (QTL) mapping of salt tolerance traits}

QTL analysis was performed to explore the genetic background of the main agronomical and salt tolerance traits measured in this study. Putative QTLs were found for all the evaluated traits, except chlorophyll content in PxRC, for both salt treatments and are shown in Figures 12 and 13 and listed in Supplementary Tables 1 and 2. Most of the traits showed a polygenic inheritance; 2-6 QTLs were identified for each trait. QTLs were identified with LOD values from 3.8-11.8 explaining from 7.5 to 57.9 of the total genetic variance. On average, the 1-LOD and 2-LOD supporting intervals spanned genetic distances of 5 and $8 \mathrm{cM}$, respectively.

Two QTLs for seed yield were identified under control conditions and three under $250 \mathrm{mM} \mathrm{NaCl}$ for each mapping population in the linkage groups 1, 2, 3, 4, 8, $10,11,16$. These QTLs had LOD values from 3.8 to 7.4 and explained from 11.8 to $37.1 \%$ of the total genetic variance. Most of the additive effects for this trait were donated by Atlas or Pasto, not Red Carina. For thousand seed weight, one QTL was found in AxRC for each treatment (on LG 3 under control conditions and LG 10 under salinity) with a LOD value of 5 and 4.3 and explaining 36 and $31 \%$ of the total variance, respectively. For PxRC TSW, 2 QTLs were found under control conditions and 5 under salinity. Several QTLs were found for plant height in both populations and treatments with high LOD values (4.0-11.3) and explaining a substantial amount of the total genetic variance (10.9-40\%). Similarly, multiple QTLs were found for $\mathrm{HI}$ and flowering time. For salt tolerance index, one QTL was found for AxRC explaining $33 \%$ of the total genotypic variance with a favourable allele from Atlas and three QTLs for PxRC with alleles donated by Pasto. For the rest of the traits (gs, SLA, RWC and chlorophyll content), alleles from both parents contributed to the observed genetic variation. Less QTLs were found in AxRC compared to PxRC, but most of them explained higher genotypic variances. A comparison of the heritabilities with the total genetic variances explained by the QTL model for each trait is depicted in Supplementary Figure 1. Stomatal conductance is a trait highly affected by the environment (VPD, humidity, light). The effect of environmental noise in gs measurements prevented the calculation of a genetic component for this trait $\left(h^{2}\right.$ was 0 for both populations and treatments). Nevertheless, a few QTLs were identified for gs, which could reflect genetic determinants found by the QTL model that could not be accounted for in the $h^{2}$ calculation, or could be one of the cases in which overfitting could not be prevented. 


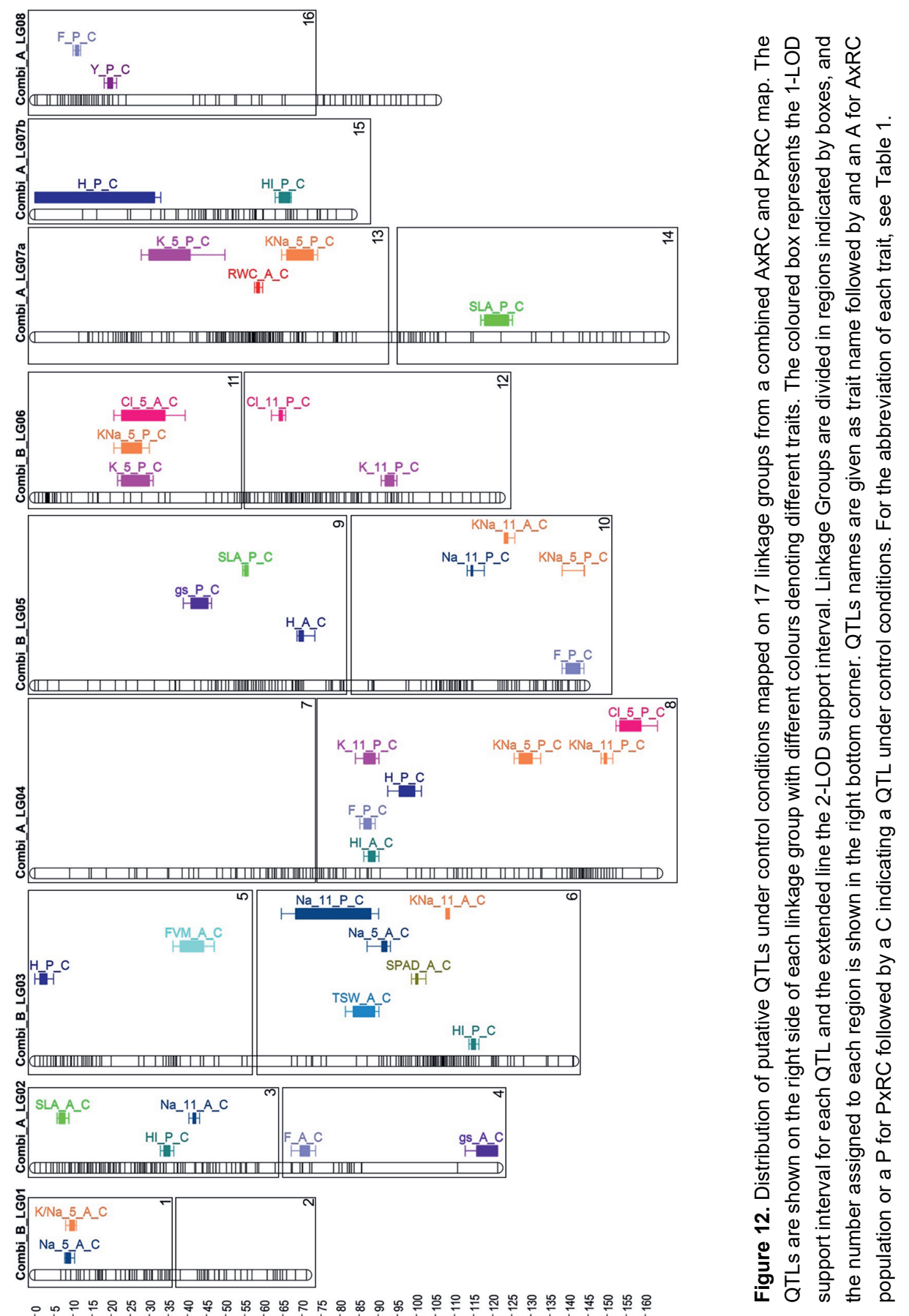



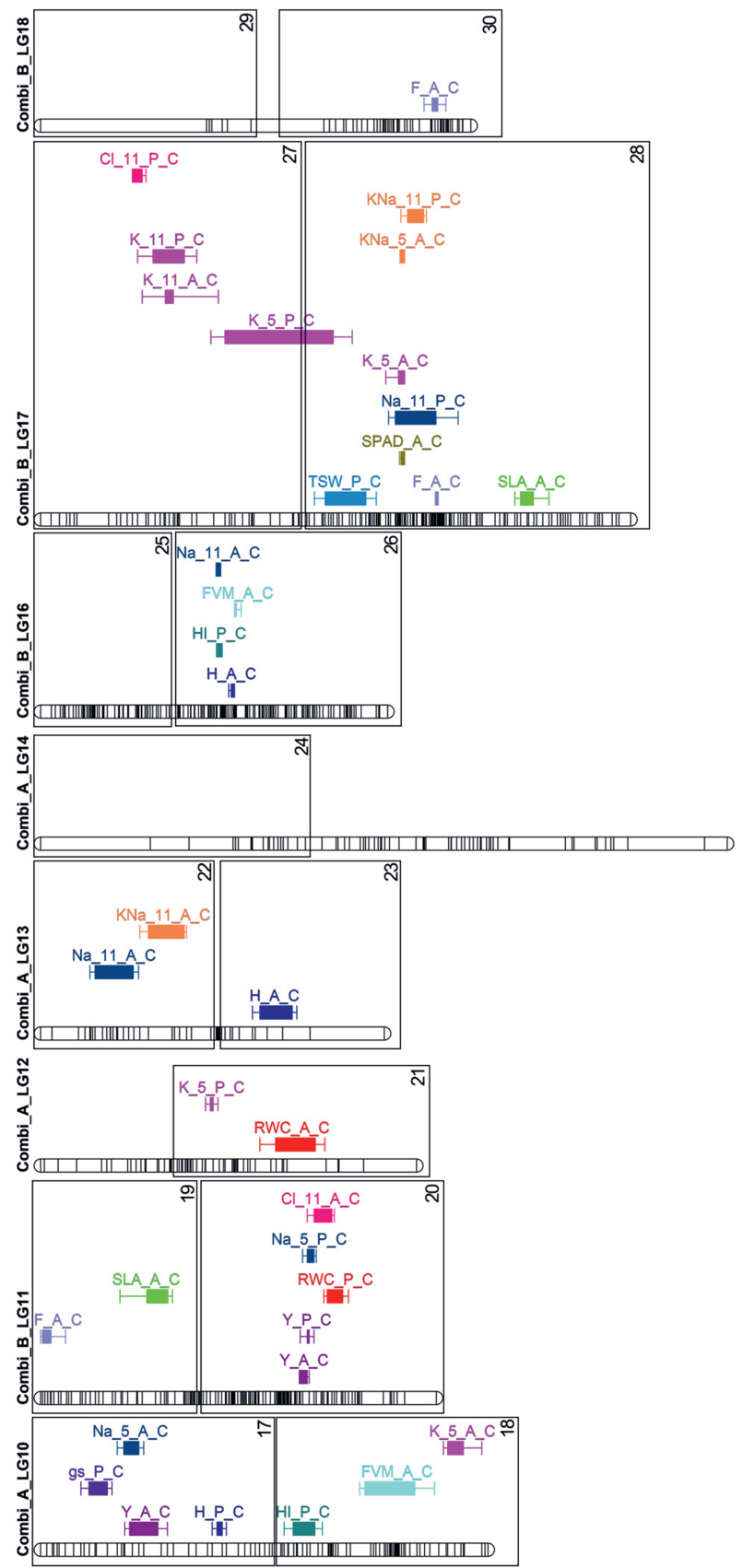

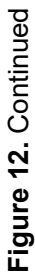

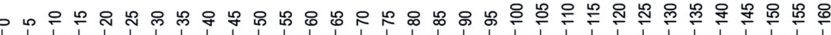

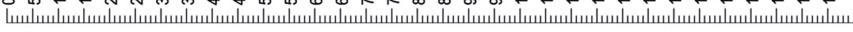



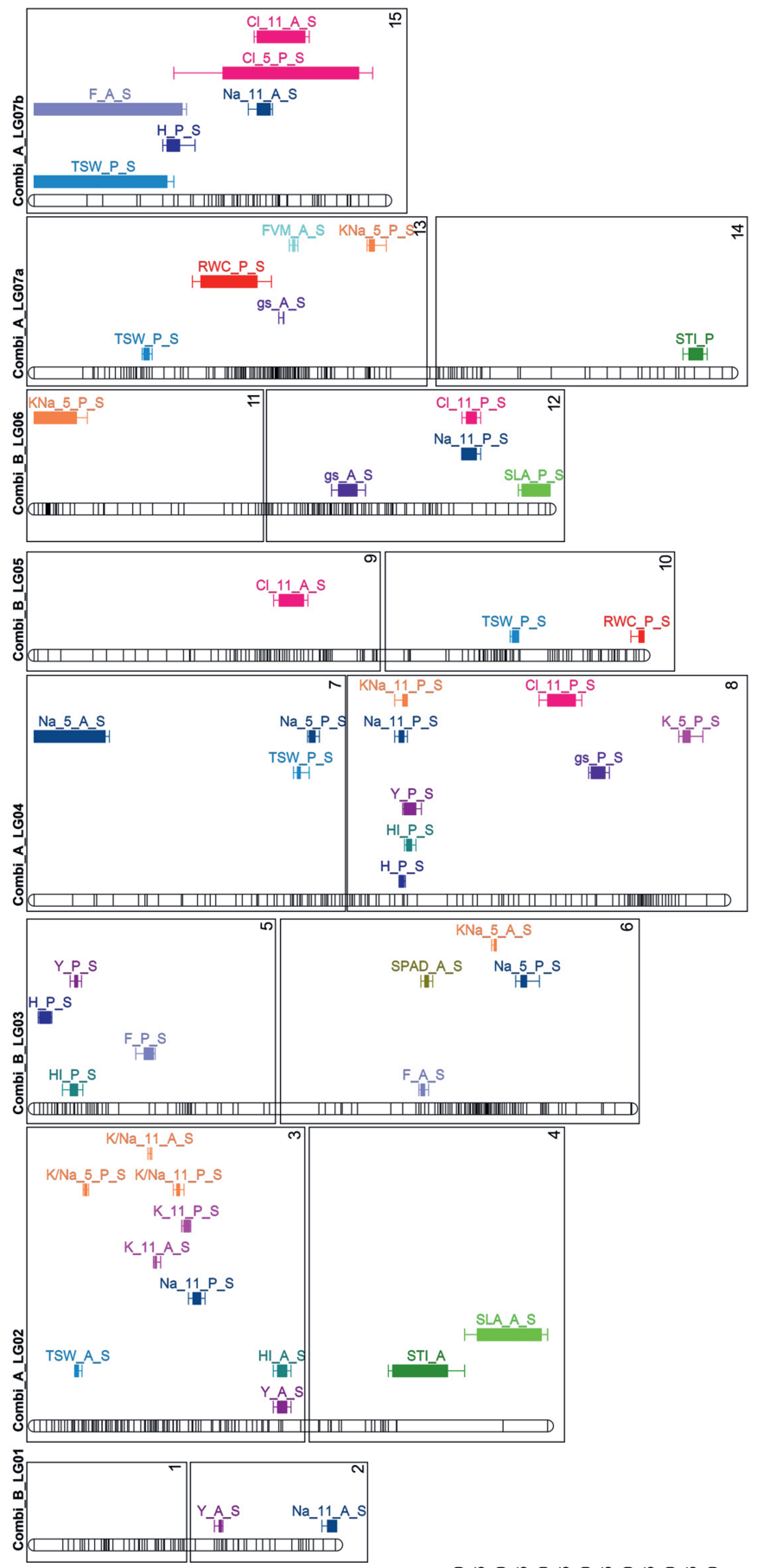

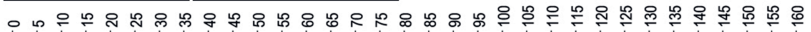

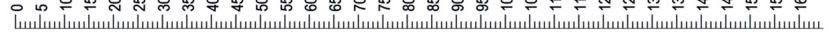

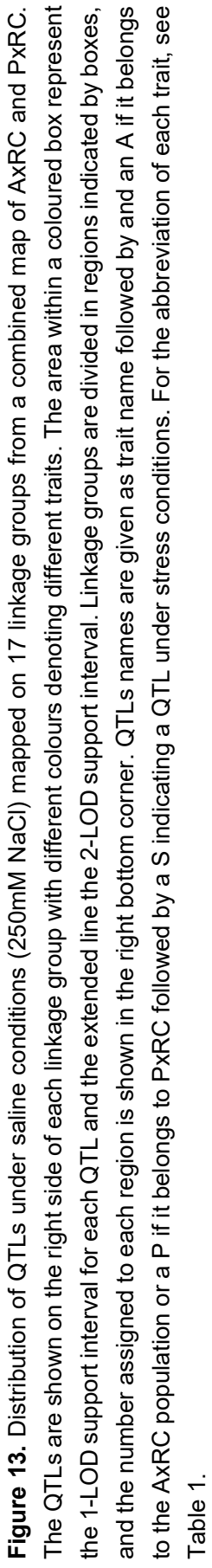



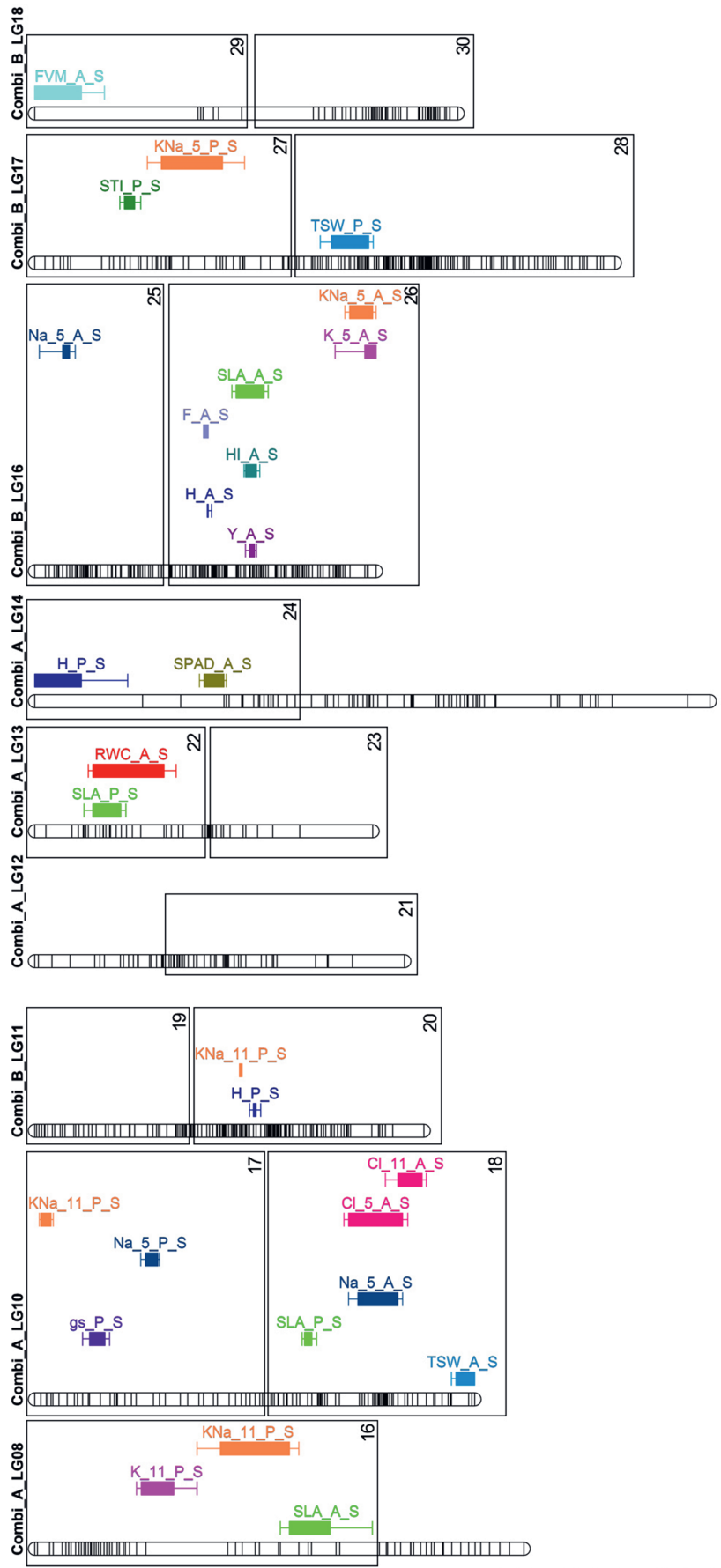

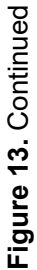

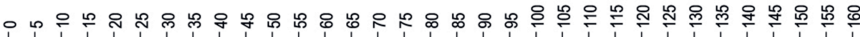

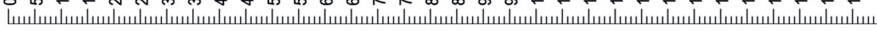


Several QTLs were identified for all the ion content measurements. For most of the ion contents, alleles from both parents contributed to the genetic variance. Of particular interest are the QTLs identified for leaf-[ $\left.\mathrm{Na}^{+}\right] 11$ weeks after start of stress, for which the large negative additive effects of the QTLs found in PxRC point to alleles related to $\mathrm{Na}^{+}$exclusion donated by Pasto. Similarly, alleles coming from Pasto appeared to contribute to the genetic variation explained by QTLs for $\mathrm{K}^{+}$and leaf $\mathrm{K}^{+} / \mathrm{Na}^{+}$found in PxRC.

To further analyse the distribution of the QTLs on the genome of quinoa, we identified several genetic regions that are of special interest (squares in Figures 12 and 13). Some of these regions accumulated QTLs for several traits, and might constitute interesting breeding targets that warrant further exploration. Under salt stress conditions, genetic regions of interest were: 1 ) region 3 , located in the upper part of linkage group Combi_A_LG02; this region contained QTLs for yield, HI, TSW, $\left[\mathrm{K}^{+}\right]$and $\mathrm{K}^{+} / \mathrm{Na}^{+}$in $\mathrm{AxRC}$; 2) region 8, located in the lower part of linkage group Combi_A_LG04, containing QTLs for yield, height, $\mathrm{HI}, \mathrm{TSW},\left[\mathrm{Na}^{+}\right]$and $\mathrm{K}^{+} / \mathrm{Na}^{+}$in PxRC; 3) region 17 in the upper part of linkage group Combi_A_LG10, containing QTLs for $g s,\left[\mathrm{Na}^{+}\right]$and $\mathrm{K}^{+} / \mathrm{Na}^{+}$in PxRC; 4) region 26 located in the lower part of linkage group Combi_B_LG16 containing QTLs for yield, height, HI, flowering, SLA, $\left[\mathrm{K}^{+}\right]$and $\mathrm{K}^{+} / \mathrm{Na}^{+}$in AxRC.. Regions accumulating multi-trait $\mathrm{QTL}$ were also identified under control conditions; for instance, region 8 contained QTLs for height, flowering, $\mathrm{HI}$ and $\left[\mathrm{K}^{+}\right]$in PxRC, and region 28 (lower part LG Combi_B_LG17) contained QTLs for flowering, SLA, chlorophyll content, $\left[\mathrm{K}^{+}\right]$and $\mathrm{K}^{+} / \mathrm{Na}^{+}$in $\mathrm{AxRC}$ and $\mathrm{TSW}$ and $\mathrm{K}^{+} / \mathrm{Na}^{+}$in PxRC.

\section{Discussion}

Quinoa is known for its high salt tolerance (Adolf et al. 2013; Hariadi et al. 2011; Jaramillo Roman et al. 2020). By evaluating the growth and yield performance of quinoa varieties and mapping populations in field-like conditions under a salt treatment of $250 \mathrm{mM} \mathrm{NaCl}$ (half strength seawater, which is considered severe salt stress level for most crops, but is only a moderate stress level for quinoa), we were able to characterize traits that contribute to the salt tolerance of quinoa under conditions that are comparable to field cultivation. Genetic variation was found for agronomical performance and for most of the assessed salt tolerance traits. The salt tolerance of the varieties based on seed yield ranged from 68 to $92 \%$. The salt tolerance of the parents of the populations were 74, 92 and $69 \%$ for Atlas, Pasto and Red Carina, respectively. The average salt tolerance was $67 \%$ for the mapping 
population AxRC and $75 \%$ for the PxRC. This indeed confirms the high salt tolerance of quinoa in general, and of the material used in this study in particular.

For a wide range of (mostly glycophytic) species, salt tolerance to moderate saline environments is linked to the ability of the plant to exclude $\mathrm{Na}^{+}$from photosynthetically active tissues (Munns 2005). High concentrations of $\mathrm{Na}^{+}$in the tissues (especially leaves) can cause a range of osmotic and metabolic disturbances in plants (Tester and Davenport 2003). In this study, the $\mathrm{Na}^{+}$content in the leaves was barely increased after 5 weeks of salinity compared to control conditions (average $44 \mathrm{mM}$ and $67 \mathrm{mM}$ at 0 and $250 \mathrm{mM} \mathrm{NaCl}$ treatment, respectively). The low concentrations of $\mathrm{Na}^{+}$measured at this time point to an extraordinary capacity of quinoa to exclude $\mathrm{Na}^{+}$from the leaves. Based on recent calculations of the amount of ions that need to be excluded to maintain osmotic adjustment (Munns et al. 2020 b), the exclusion of $\mathrm{Na}^{+}$observed in this study should be higher than $99 \%$, which is much higher than in many other reported plant species. Towards the end of the growing cycle, [ $\left.\mathrm{Na}^{+}\right]$accumulated in the leaves (average $45 \mathrm{mM}$ and $422 \mathrm{mM}$, at 0 and $250 \mathrm{mM} \mathrm{NaCl}$, respectively). Pasto had the lowest levels of $\mathrm{Na}^{+}$compared to the other varieties throughout the entire cycle. This was also reflected in the populations: $\left[\mathrm{Na}^{+}\right]$in the leaves of PxRC was on average lower than in AxRC (391 $\mathrm{mM}$ in PxRC and $520 \mathrm{mM}$ in $\mathrm{AxRC}$ ). In a number of other species, $\mathrm{Na}^{+}$accumulation in the shoot was shown to be under strict genetic control with most of its genetic variation attributable to additive effects (Flowers 2004). Quinoa in our experiment was no exception; $\mathrm{Na}^{+}$content in the leaves at 11 weeks after start of the stress showed rather high levels of heritability $(63 \%$ and $71 \%$ for AxRC and PxRC, respectively), and several QTLs were identified for $\left[\mathrm{Na}^{+}\right]$that explained $60 \%$ of the genetic variation of each population. In particular, the QTLs identified in PxRC had large negative additive effects, pointing to alleles donated from Pasto possibly underpinning transporters of regulators associated to the high $\mathrm{Na}^{+}$exclusion from the shoot measured in this variety.

After 5 weeks of stress, the average $\left[\mathrm{K}^{+}\right]$in salt stressed plants was $20 \%$ higher than in control plants. $\left[\mathrm{K}^{+}\right]$in leaves was not significantly different between treatments for most of the evaluated genotypes, with the exception of Pasto, for which $\left[\mathrm{K}^{+}\right]$concentration was $53 \%$ increased by the salt treatment. Differences in $\left[\mathrm{K}^{+}\right]$were also found between the populations; PxRC had a higher leaf $\left[\mathrm{K}^{+}\right]$than AxRC. It has been reported that a distinctive response of quinoa to saline environments is its unique capacity to maintain or even increase $\mathrm{K}^{+}$levels in the shoots (Hariadi et al. 2011; Jaramillo Roman et al. 2020; Schmöckel et al. 2017). For 
the same quinoa varieties, we previously observed that severe salt stress $(400 \mathrm{mM}$ $\mathrm{NaCl}$ or higher) actively increased the $\left[\mathrm{K}^{+}\right]$in the shoot to levels considerably higher than the controls (Jaramillo Roman et al. 2020). The active increase of $\mathrm{K}^{+}$seems to be a survival mechanism of quinoa to severe and prolonged stress, while $\mathrm{K}^{+}$ retention (as observed in this experiment) might be the preferred mechanism at mild or short-duration stress. These differences might be related with the high metabolic costs of selecting and transporting $\mathrm{K}^{+}$in the plants from a growing medium that has a high concentration of $\mathrm{Na}^{+}$ions (Rubio et al. 2020). Despite the ability of quinoa to retain $\mathrm{K}^{+}$, leaf $\left[\mathrm{K}^{+}\right]$segregated in both populations and wide genetic diversity was observed for this trait to which multiple QTLs contributed. In PxRC, [K+] had a positive correlation with seed yield and salt tolerance, while in AxRC it was negatively correlated with seed yield. This demonstrates that the populations used different strategies in response to salinity. The QTLs identified for PxRC had large positive additive effects pointing to alleles donated by Pasto, possibly associated with the higher $\left[\mathrm{K}^{+}\right]$and a strategy that prioritizes survival that characterizes this variety (Chapter 2 and 4).

The $\mathrm{K}^{+} / \mathrm{Na}^{+}$ratio in young leaves was not significantly reduced for the commercial varieties or the mapping populations in the first five weeks of salt treatment. Towards maturity, the accumulation of $\mathrm{Na}^{+}$in the shoot with no further increase of $\mathrm{K}^{+}$resulted in a significantly reduced $\mathrm{K}^{+} / \mathrm{Na}^{+}$ratio in salt-stressed plants, except for Pasto, which maintained a relatively high $\mathrm{K}^{+} / \mathrm{Na}^{+}$ratio (3.6 after 11 weeks of salt stress). Reports in several species demonstrate that the $\mathrm{K}^{+} / \mathrm{Na}^{+}$ratio is heritable, and might be controlled by several genes (Cuartero et al. 2006; Maathuis and Amtmann 1999). In our study, the average $h^{2}$ for the leaf $\mathrm{K}^{+} / \mathrm{Na}^{+}$ratio under stress for both timepoints and populations was low (30\%). In spite of this, several QTLs were found for $\mathrm{K}^{+} / \mathrm{Na}^{+}$under salinity that explained up to $40 \%$ of the genetic variance. As can be expected, most of these QTLs co-localized with QTLs for $\mathrm{K}^{+}$ (genetic region 16 for $\mathrm{PxRC}$, and 26 for $\mathrm{AxRC}$ ), $\mathrm{Na}^{+}$(genetic regions 8 and 17 for $\mathrm{PxRC}$, and 6 for AxRC) or both ions (genetic region 3 for PxRC). QTLs for $\mathrm{Na}^{+}, \mathrm{K}^{+}$ and/or $\mathrm{K}^{+} / \mathrm{Na}^{+}$ratio were also found in the same genetic regions for both populations (genetic regions 3 and 6 ) and in both treatments (regions 6 and 8). The colocalization of these QTLs indicates that $\mathrm{Na}^{+}$and $\mathrm{K}^{+}$homeostasis is linked. In most plant species, $\mathrm{Na}^{+}$and $\mathrm{K}^{+}$are negatively correlated. In quinoa, especially at high salt concentrations, $\mathrm{K}^{+}$is positively correlated with $\mathrm{Na}^{+}$, which makes this an exceptional trait that may contribute to its resilience. We identified genetic regions in the genome with one or more genes controlling ion transport or ion homeostasis in quinoa. As an example, genetic region 3 (upper part LG Combi_A_LG02) contains a QTL with a 
strong negative additive value for $\mathrm{Na}^{+}$and QTLs with positive additive values for $\mathrm{K}^{+}$ and $\mathrm{K}^{+} / \mathrm{Na}^{+}$in PxRC. It is possible that this region contains one or more alleles donated by Pasto that might contribute to a phenotype with low $\mathrm{Na}^{+}$content, high $\mathrm{K}^{+}$ retention, and consequently high $\mathrm{K}^{+} / \mathrm{Na}^{+}$ratio.

In contrast to $\mathrm{Na}^{+}, \mathrm{Cl}^{-}$concentration in leaves increased strongly from early stages in the growth cycle in salt-stressed quinoa plants. At the onset of flowering, the average $\left[\mathrm{Cl}^{-}\right]$was $189 \mathrm{mM}$ under salinity and it reached $313 \mathrm{mM}$ towards maturity. $\mathrm{Cl}^{-}$could serve as a cheap osmolyte for osmotic adjustment and later in the season, when the concentration of $\mathrm{Na}^{+}$rises, it might have an additional function to balance electrical charge of monovalent positive ions $\left(\mathrm{Na}^{+}\right.$and $\mathrm{K}^{+}$) (Teakle and Tyerman 2010). Some genotypes of the populations reached much higher levels of $\mathrm{Cl}^{-}$compared to the varieties, and the average ${ }^{\left[\mathrm{Cl}^{-}\right]}$was higher in the progeny of AxRC (490 mM) compared to PxRC (380 mM). The [ $\left.\mathrm{Cl}^{-}\right]$in PxRC was positively correlated to seed yield, HI, TSW, suggesting that some genotypes from PxRC with higher $\mathrm{Cl}^{-}$contents in the shoots may have allocated resources more effectively to seeds (higher seed yield, $\mathrm{HI}, \mathrm{TSW}$ ). AxRC showed higher concentrations of $\mathrm{Cl}^{-}$in the leaves, but for this population [ $\left.\mathrm{Cl}^{-}\right]$was negatively correlated to STI, TSW, flowering and gs. It is possible, that accumulating $\mathrm{Cl}^{-}$might be a useful salt stress response for quinoa until a certain threshold, while above that level the toxicity of $\mathrm{Cl}^{-}$ or the metabolic cost of compartmentalization of this ion might restrict the growth and performance of the plants. Although $\mathrm{Cl}^{-}$was strongly correlated to $\mathrm{Na}^{+}$, the latter did not correlate with agronomical traits in AxRC; possibly the impact of $\mathrm{Cl}^{-}$on the plant performance of AxRC is independent of the accumulation of $\mathrm{Na}^{+}$. Similar to reports from other species (Flowers 2004), we found high heritabilities for [ $\left.\mathrm{Cl}^{-}\right]$under salinity (66 \% and $72 \%$ for AxRC and PxRC, respectively) and we identified several QTLs explaining from 17 to $31 \%$ of the total genetic variance. In agreement with the correlation patterns found in this study, QTLs for $\mathrm{Cl}^{-}$under salinity colocalized with QTLs for other traits. The genetic region 8 (Figure13) appears to be a hotspot for QTLs in PxRC that deserves further exploration. This region contains QTLs for $\mathrm{Na}^{+}$, $\mathrm{Cl}^{-}, \mathrm{K}^{+} / \mathrm{Na}^{+}$, gs, plant height, yield and harvest index. Alleles donated by Pasto in this region appear to contribute to lower $\mathrm{Na}^{+}$and $\mathrm{Cl}^{-}$contents, and higher $\mathrm{K}^{+} / \mathrm{Na}^{+}$, gs and plant height while alleles donated by Red Carina might point to higher yield and harvest index. This region might be an interesting breeding target to for improvement of salt tolerance. It remains to be established whether the colocalization of these QTLs point to several tightly-linked genes clustering in the same genetic regions, or 
pleiotropic effects of a gene influencing several traits that results in a particular salt stress response phenotype.

Stomatal closure is a common water management strategy under salinity that at the same time decreases the transport rate of ions towards the leaves (Shabala 2013). Up to a certain threshold, a reduction in stomatal conductance may have an adaptive advantage under saline conditions, saving water, thus improving plant water use efficiency (Chaves et al. 2009). Research has been done to define the threshold below which gs limits photosynthesis; even though this parameter is highly influenced by environmental factors (e.g. VPD) and varies considerably between species, a gs close to $150 \mathrm{mmol} / \mathrm{m}^{2} \mathrm{~s}$ has been proposed as the minimum gs that does not impair C3 photosynthesis (Medrano et al. 2002). The average gs under salinity in this study was $148 \mathrm{mmol} / \mathrm{m}^{2} \mathrm{~s}$ (33\% reduction from the control) while the average yield reduction was $25 \%$. This might indicate that partial closure of stomata in quinoa is an important trait that enables quinoa to grow and yield well under what is considered for most crops high salinity. Other parameters associated with photosynthesis (chlorophyll content and the initial light use efficiency of PSII (Fv/Fm)) were also unaffected by the salt treatment, indicating that photosynthetic efficiency was hardly affected in the plants.

In chapter 2, we identified that a reduction of SLA is one of the main adaptations of quinoa to severe salt stress ( $\geq 400 \mathrm{mM} \mathrm{NaCl}$ ) (Jaramillo Roman et al. 2020). The formation of thicker and smaller leaves has several advantages for the plants: it reduces the transpiration area and improves WUE. In addition, thicker leaves have a higher water content which helps to dilute the concentration of ions accumulated under severe salt exposure (Jaramillo Roman et al. 2020) (see also Chapter 4). Under moderate salinity, the SLA of the commercial varieties Atlas, Jessie and Red Carina was slightly increased. For the mapping populations, SLA was not significantly different between treatments for the progeny of PxRC and was slightly increased by salt for AxRC. As depicted in the PCA (Figure 9), AxRC, together with Atlas and Red Carina, were grouped in one cluster based on their higher SLA that was negatively correlated to gs, chlorophyll content, Fv/Fm and salt tolerance index, variables that contributed to the clustering of PxRC and Pasto in a separate group. The interaction between these physiological traits suggest that the regulation of SLA is also a response of quinoa to moderate salt stress; but seems to be less crucial than under high salinity. Two of the putative QTLs identified for SLA in PxRC had large negative additive values, which points to alleles donated by Pasto possibly associated with lowered SLA. It is possible that these QTLs might play an 
even more important role for the tolerance of quinoa under high salt stress levels (see Chapter 2).

By studying two biparental populations and six commercial varieties we were able to compare salt tolerance mechanisms in a wide range of genotypes and the inheritance of salt tolerance traits between and within mapping populations. All the varieties were highly salt tolerant but Pasto showed the highest salt tolerance index. The main physiological adaptations of Pasto to salt stress were the formation of slightly thicker leaves (lower SLA), higher RWC, and higher SPAD. In addition, this variety excluded more $\mathrm{Na}^{+}$from leaves throughout the whole season, retained more $\mathrm{K}^{+}$, and maintained a high $\mathrm{K}^{+} / \mathrm{Na}^{+}$ratio. Most of these traits were also observed in the progeny of PxRC, and, in comparison with AxRC, it showed a higher salt tolerance. We found QTLs for all the analysed traits and identified putative alleles donated by Pasto that contributed to lower $\mathrm{Na}^{+}$and $\mathrm{Cl}^{-}$contents, higher $\mathrm{K}^{+}$retention and lower SLA of the PxRC population. From this study, PxRC looks like a promising population to study several salt tolerance traits. Finally, despite of the superior STI of Pasto and PxRC, it is important to note that Atlas and AxRC showed higher yields under control and salt stress conditions. Several QTLs for yield and yield-related traits were found for this population in both treatments and many alleles donated by Atlas could be identified. Incorporating these alleles together with salt tolerance traits alleles donated by Pasto might be a possibility to breed for superior quinoa genotypes for yield and salt tolerance.

\section{Implications for breeding}

In addition to salt tolerance traits, the genetic variation for some of the most important breeding targets of quinoa was evaluated in this study: seed yield, TSW, flowering time and harvest index (López-Marqués et al. 2020). For both populations, flowering time was positively correlated with plant height which in turn had a negative correlation with harvest index: late varieties tend to grow more and become taller, while they allocate less resources into seed production. The parents of both populations showed contrasting phenotypes for these traits and transgressive segregation was found for all of them. RedCarina is a late flowering variety compared to Pasto and Atlas and it is also the tallest one; Pasto is the lowest yielding variety while Atlas is the highest one. Some of the QTLs identified in this study could be potentially useful breeding targets. For example, QTLs related to flowering time were identified in genetic regions 4, 19 and 30 for AxRC with alleles donated by Atlas that might relate to early flowering. In region 16, QTLs for yield and flowering time were 
identified in PxRC, possibly an allele donated by Pasto contributed to early flowering and an allele donated by RedCarina contributed to higher yield in the progeny.

Developing breeding tools and identifying new breeding targets are essential steps to optimize and accelerate the breeding of quinoa. The markers developed with the novel single primer enrichment technology (SPET) (NUGEN Technologies, San Carlos, CA, USA) used in this paper for genotyping a quinoa mapping population, together with the combined genetic map for PxRC and AxRC provide important tools for the dissection of the genetic architecture of agronomical and abiotic stress tolerance traits and the advancement of quinoa as a food crop with high potential.

\section{Acknowledgments}

We gratefully acknowledge the expert assistance of Rohan van Genderen and Rinnie Verwoert in the tunnel experiment. Viviana Jaramillo is financially supported by a scholarship from the Secretariat for Higher Education, Science, Technology and Innovation of the Republic of Ecuador (Contract Number 2015AR2Q8878). 


\section{Supplementary material}

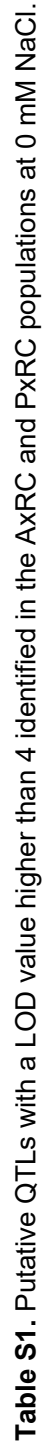

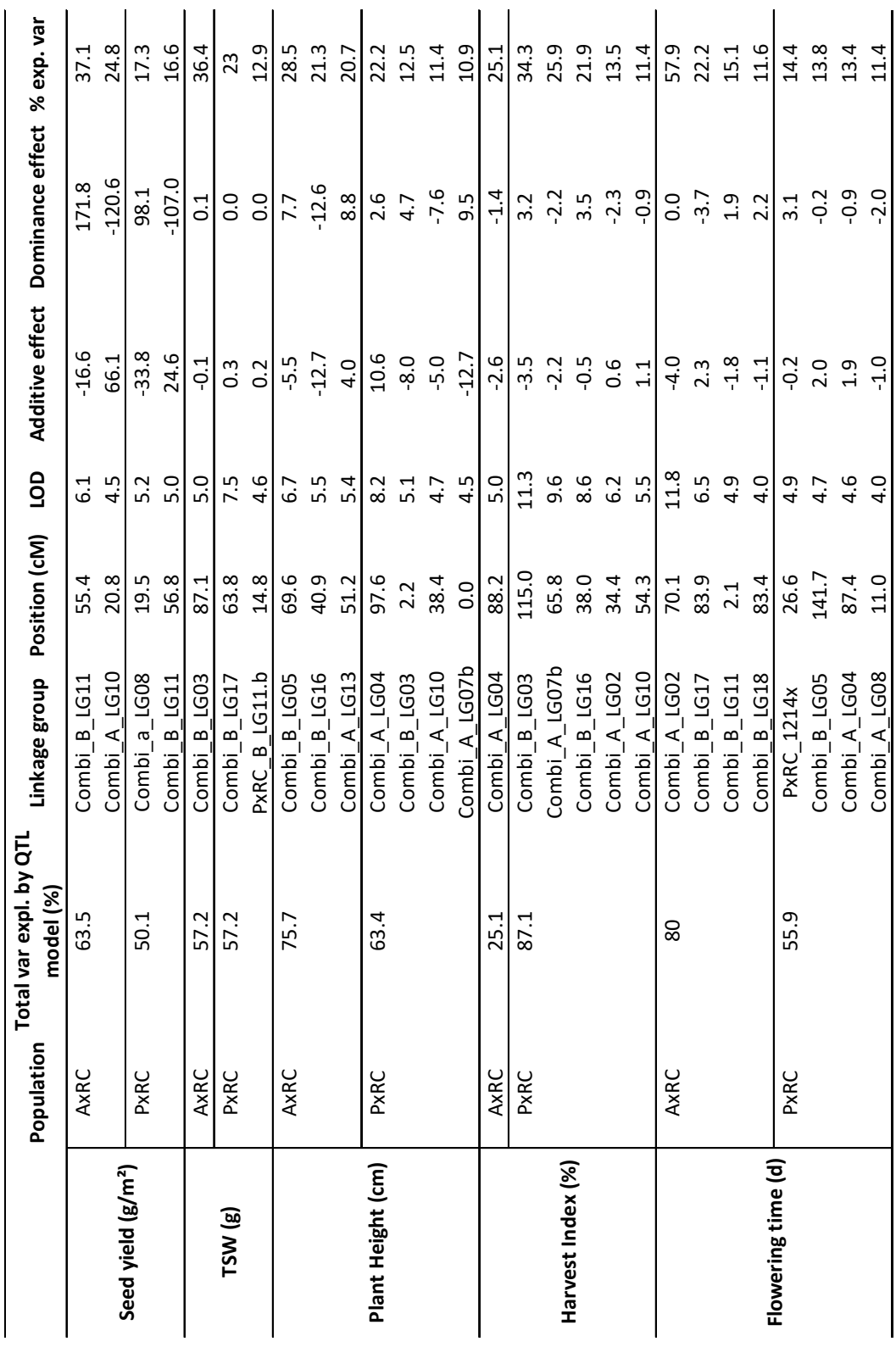




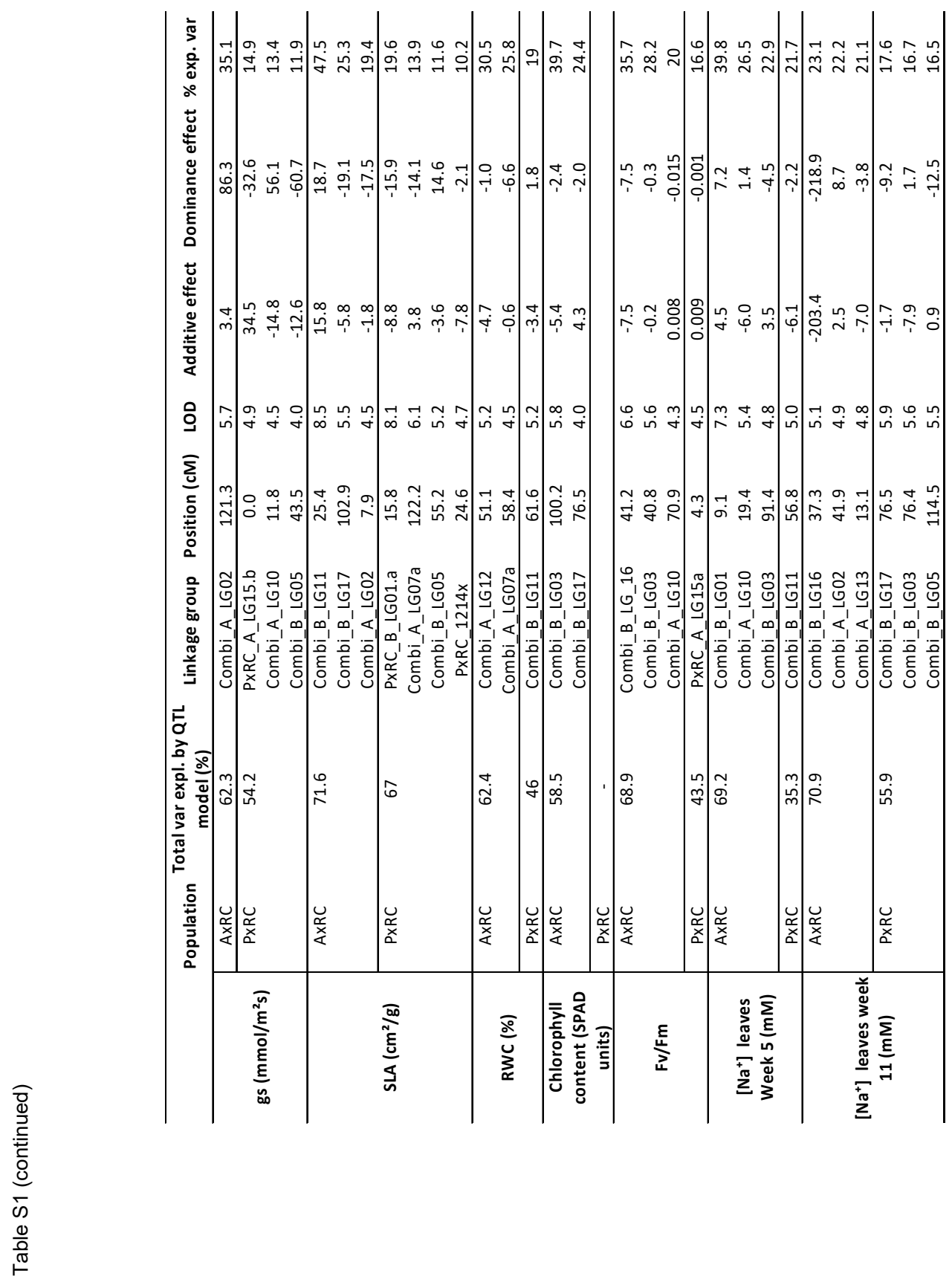




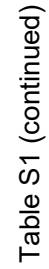

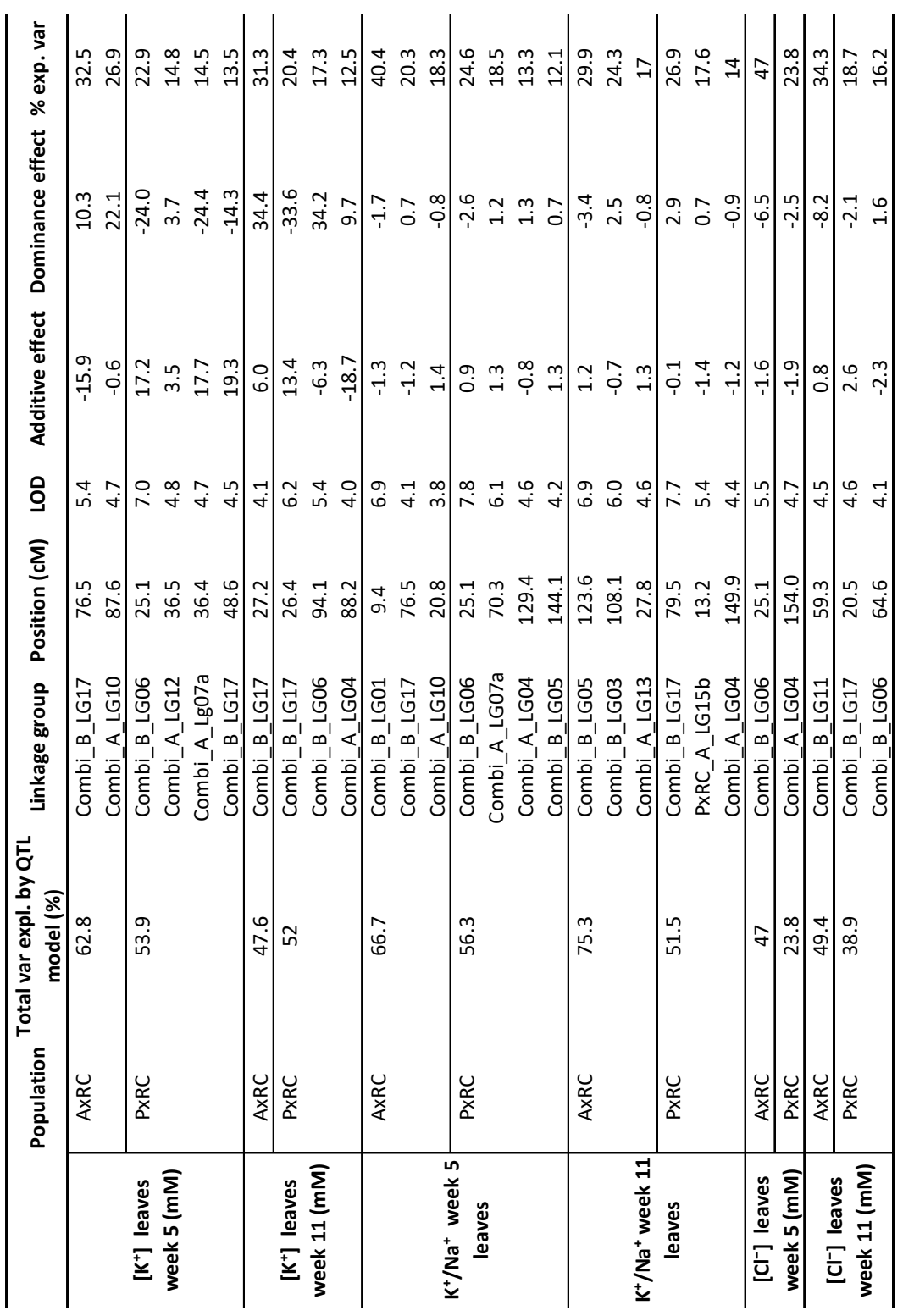




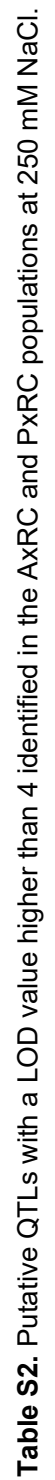

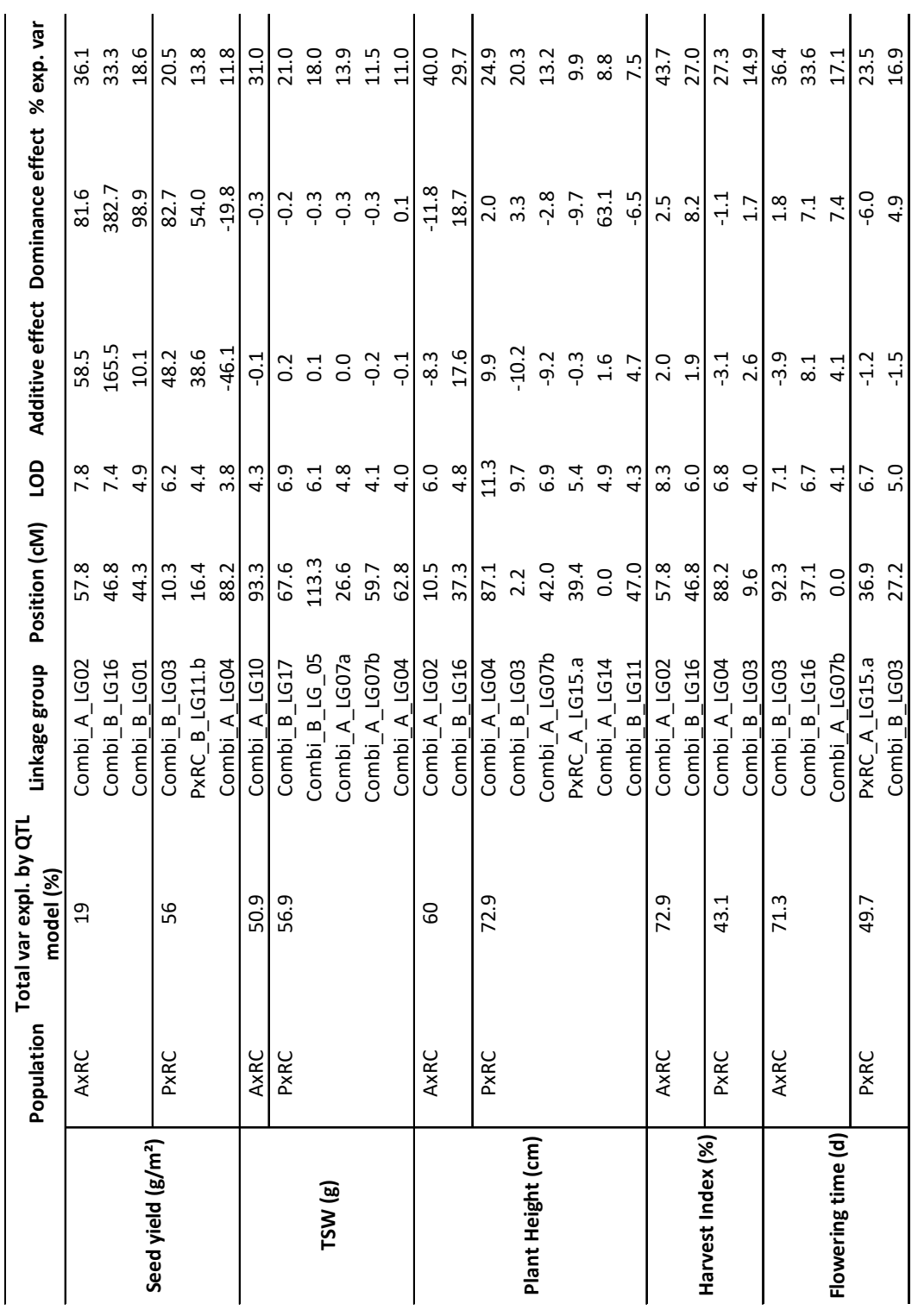




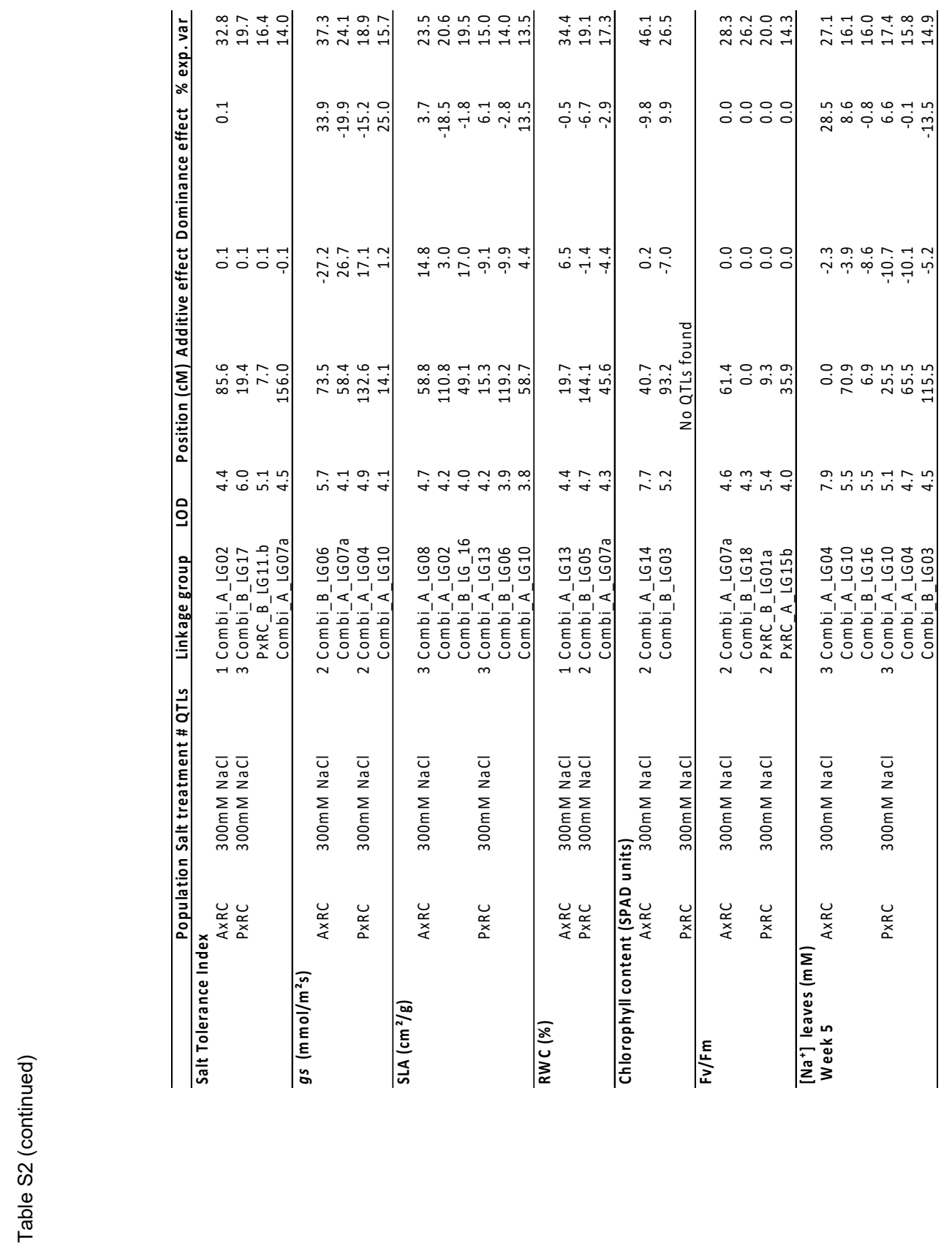




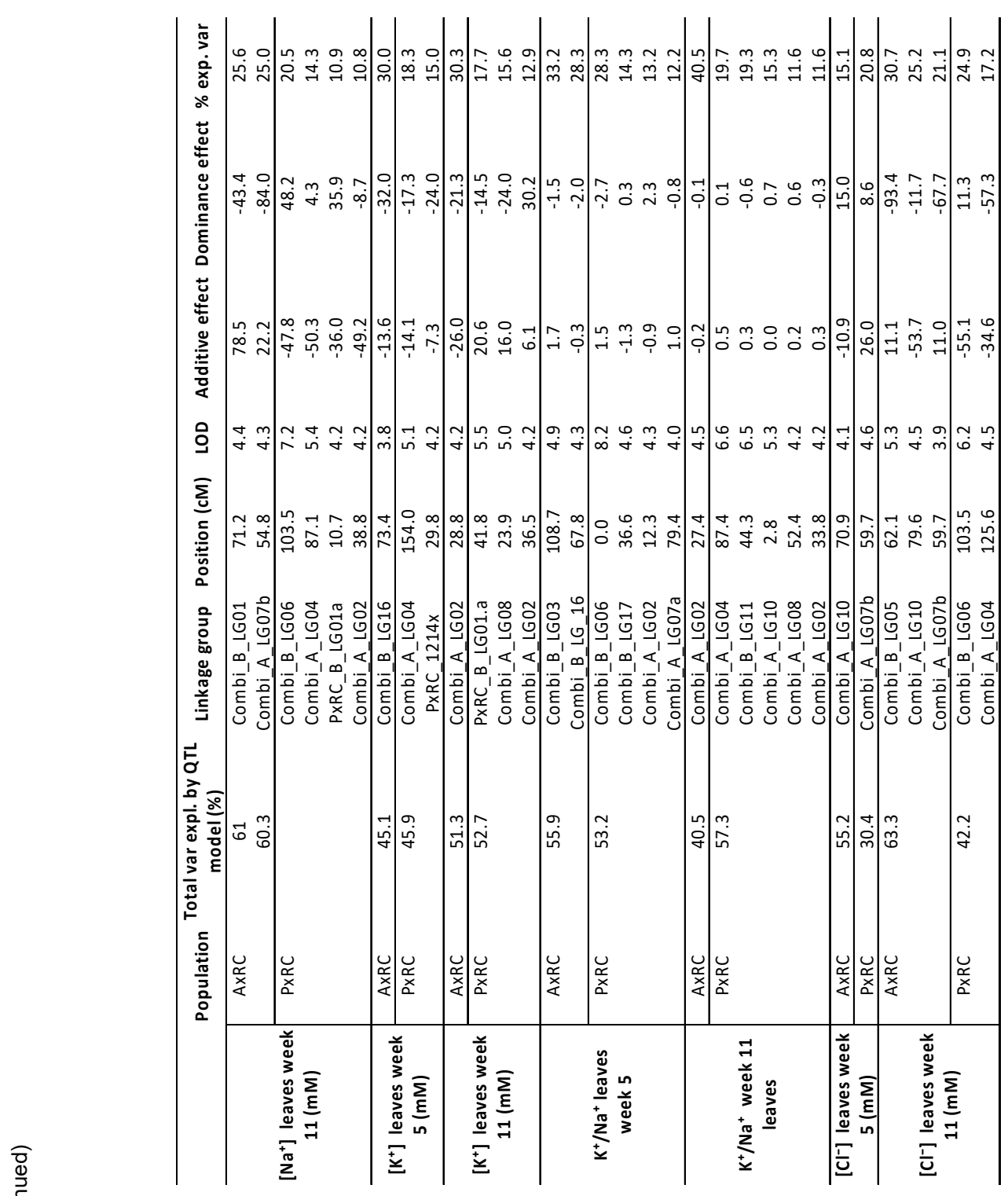




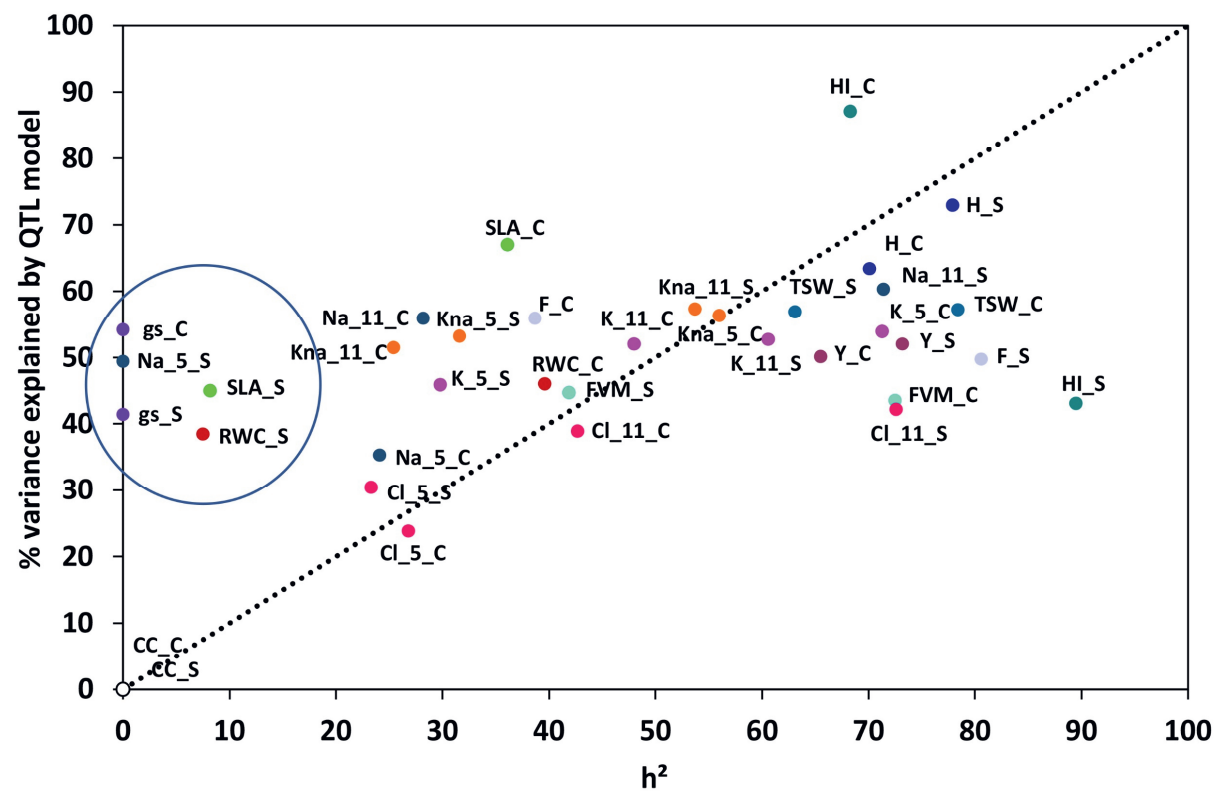

Figure S1. Comparison between $h^{2}$ and the total genetic variance explained by the QTL model of each trait measured for PxRC under control and stress conditions. The blue circle indicates traits for which it was not possible to avoid overfitting 


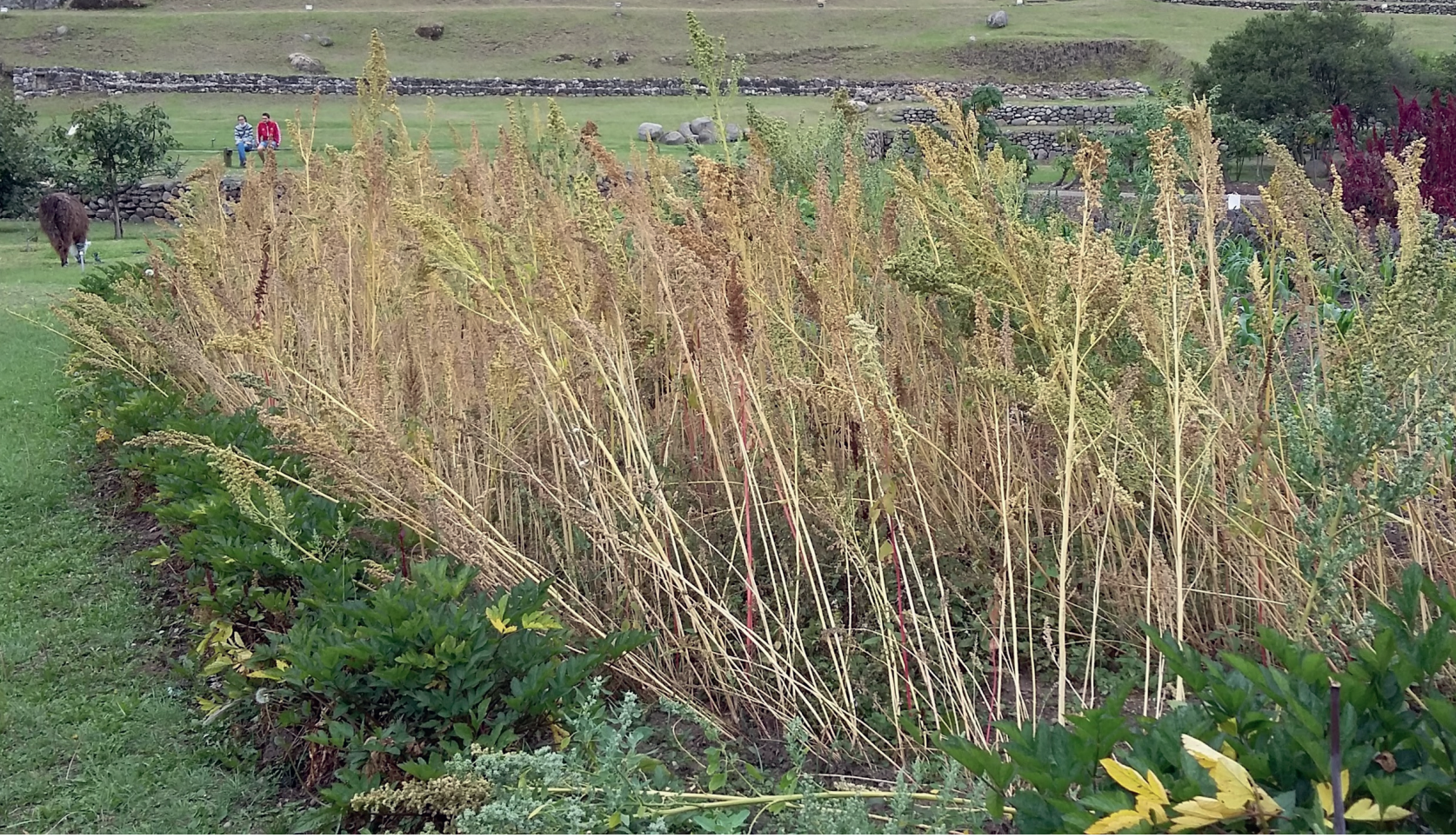

\section{Chapter 6}

Genetic diversity of Inter-Andean Valley Ecuadorian quinoa for salt tolerance

Viviana Jaramillo Roman, Yuxiao Zeng, Hanneke van der Schoot, Andres F. Torres, Maria de Lourdes Torres, Richard G.F. Visser, Eibertus N. van Loo, C. Gerard van der Linden 


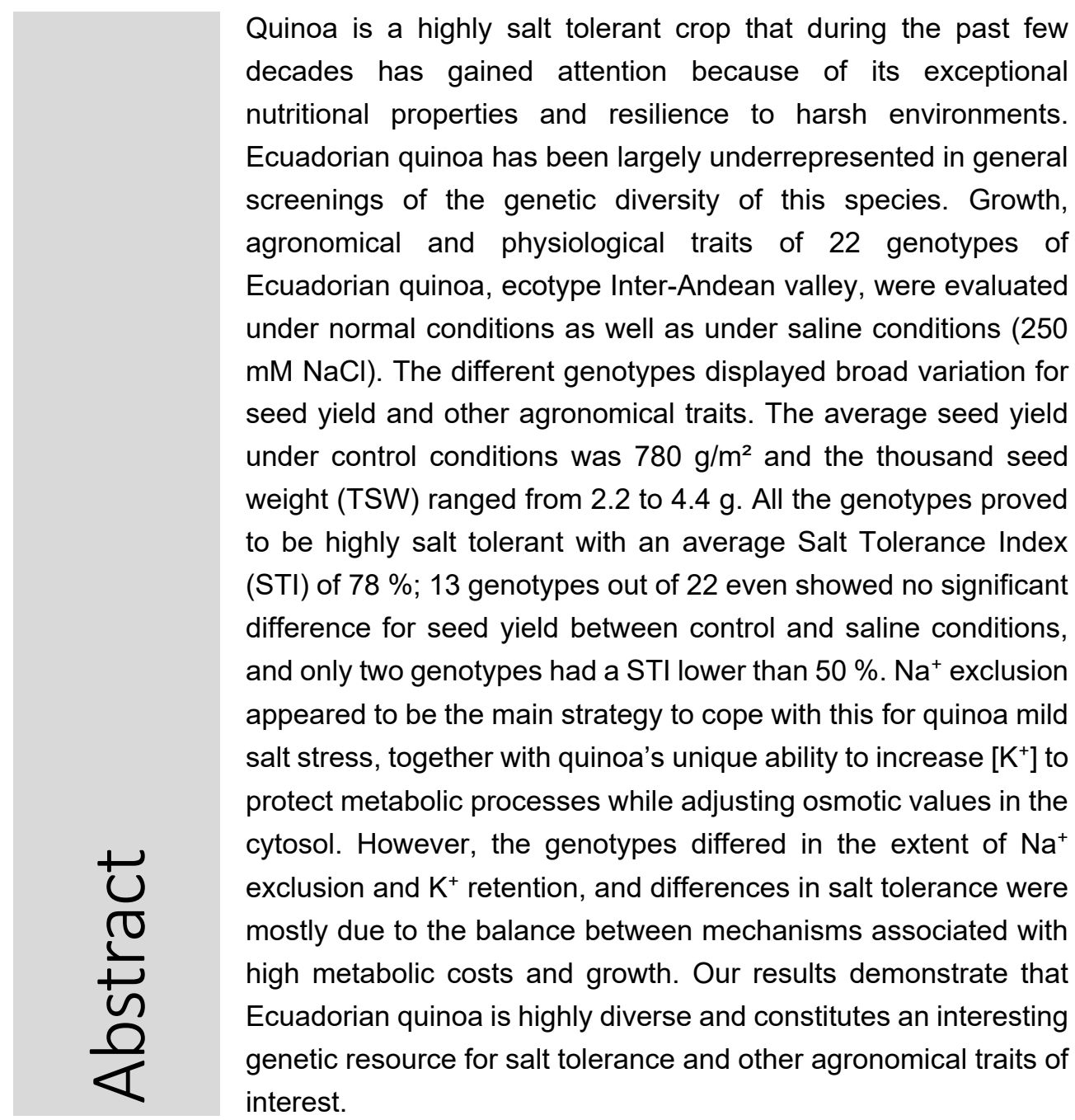




\section{Introduction}

Soil salinity is a major constraint for agricultural production. Almost $20 \%$ of the global arable land and more than 75 countries are adversely affected by soil salinization, and the productivity losses caused by salt stress are high (Qadir et al. 2014). Improving salt tolerance of crops s a sustainable strategy to counteract the negative effects of salinity on agricultural production. In addition, this would open up the possibility to use brackish or saline water for irrigation to relieve the growing pressure on freshwater resources (Morton et al. 2019).

Salt tolerance can be defined as the ability of plants to grow and produce yield in soils affected by salinity. The degree of salinity that plants can tolerate depends on the species, duration, and stage of crop development when exposed to salinity (Munns et al. 2019). Plants vary widely in their response to salt stress, but key traits contributing to salt tolerance have been defined: the ability to restrict the rate of entry of potentially toxic $\mathrm{Na}^{+}$and $\mathrm{Cl}^{-}$into the shoot ('ion exclusion') (Tester and Davenport 2003), the maintenance of $\mathrm{K}^{+}$uptake (' $\mathrm{K}^{+}$retention') (Wu et al. 2018), the accumulation of solutes in the cytosol to decrease water potential in the external media and maintain cell turgor ('osmotic adjustment') (Flowers et al. 2015), the compartmentalization of toxic ions in the vacuole or accumulation in older tissues rather than in photosynthetically active leaves ('tissue tolerance') (Deinlein et al. 2014), and the ability to increase water use efficiency ('transpiration efficiency') (Shabala 2013).

As such, salt tolerance is dependent on a number of traits and presumably controlled by many genes. It has been argued that the best way to improve and breed for salt tolerance should rely on the screening of the diversity within crop species, the identification of the underlying traits associated with salt tolerance and the 'pyramiding' of these traits as a breeding strategy, rather than the selection of 'salt tolerance' as a trait per se (Colmer et al. 2005).

Often, little variation for salt tolerance is available in commercial varieties of crops and therefore these may not be the best-suited germplasm to breed for varieties that can be cultivated in challenging environments (Cheng 2018). For this reason, new sources of variation, including the use of non-elite varieties, landraces and wild relatives, should be considered as genetic resources and donors for salt tolerance traits to improve salinity tolerance of crops (Roy et al. 2011).

A further alternative to expand our knowledge about salt tolerance and breed for sustainable agriculture is to increase crop diversification. Many of the world's underutilised crops possess resilience traits and have the ability to withstand abiotic 
and biotic stresses to a greater extent than the current major staple crops (Cheng 2018). A potential new crop that has gained ground in the last few decades is quinoa (Chenopodium quinoa). Quinoa is a pseudo-cereal considered to be an ideal food source, being gluten-free, protein-rich and containing all nine essential amino acids. It has high genetic and phenotypic diversity and is unique in its ability to grow well under normal conditions, while also displaying remarkably high tolerance to several abiotic stresses, including salinity (Bazile et al. 2016a); quinoa is considered a facultative halophyte. Quinoa originated in the Andean region near Lake Titicaca in Peru and Bolivia. Based on its diversification from the centre of origin and its adaptation to specific environments, five ecotypes were classified: Highlands (Peru and Bolivia), Inter-Andean valley (Colombia, Ecuador and Peru), Salares (salt flats on the Andean High plateau in Bolivia, Chile and Argentina), Yungas (a warm, rainy and humid stretch of forest on the slope of the Andes mountains, Bolivia) and Costal/Lowlands (Chile) (Bazile et al. 2016a; Fuentes et al. 2012).

Despite the growing attention for quinoa as a food crop, the genetic diversity of the species remains largely unexplored. The main issue limiting this exploration is the lack of facilitated access to genetic resources for both research and commercialization, added to the difficulty of access to quality seed (Bazile et al. 2016a). Ecuadorian germplasm is particularly underrepresented in general screenings of the molecular and agronomical diversity of quinoa, and in our previous research we aimed to construct a core collection of Ecuadorian quinoa germplasm that is cultivated by small farmers from the Andean provinces of Ecuador (Salazar et al. 2019).

In this study we explore the genetic diversity of agronomical and physiological traits in 22 genotypes from our collection of Ecuadorian quinoa, and evaluate their strategies to cope with salinity. Our results show that Ecuadorian germplasm is highly diverse and different genotypes rely on different mechanisms to tolerate salinity, offering opportunities for a better understanding of the remarkable salt tolerance of this facultative halophyte species. 


\section{Materials and Methods}

\subsection{Plant Material}

Seeds of 84 quinoa genotypes were collected from small farmers throughout the highlands of Ecuador (See also (Salazar et al. 2019)). The material was propagated in The Netherlands, but viable seeds were obtained only from 40 genotypes. The low propagation success was attributed to low quality seed or late maturity of photoperiod-sensitive cultivars (Christiansen et al. 2010). From the genotypes from which viable seed was obtained, 22 were selected that represent most of the genetic diversity as determined (Salazar et al. 2019). Detailed information about these genotypes is presented in Table 1 and their collection-sites in the Ecuadorian highlands is shown in Figure 1. Two Dutch quinoa varieties (Pasto and Red Carina) from Plant Breeding, Wageningen University \& Research (The Netherlands), were included as reference varieties.

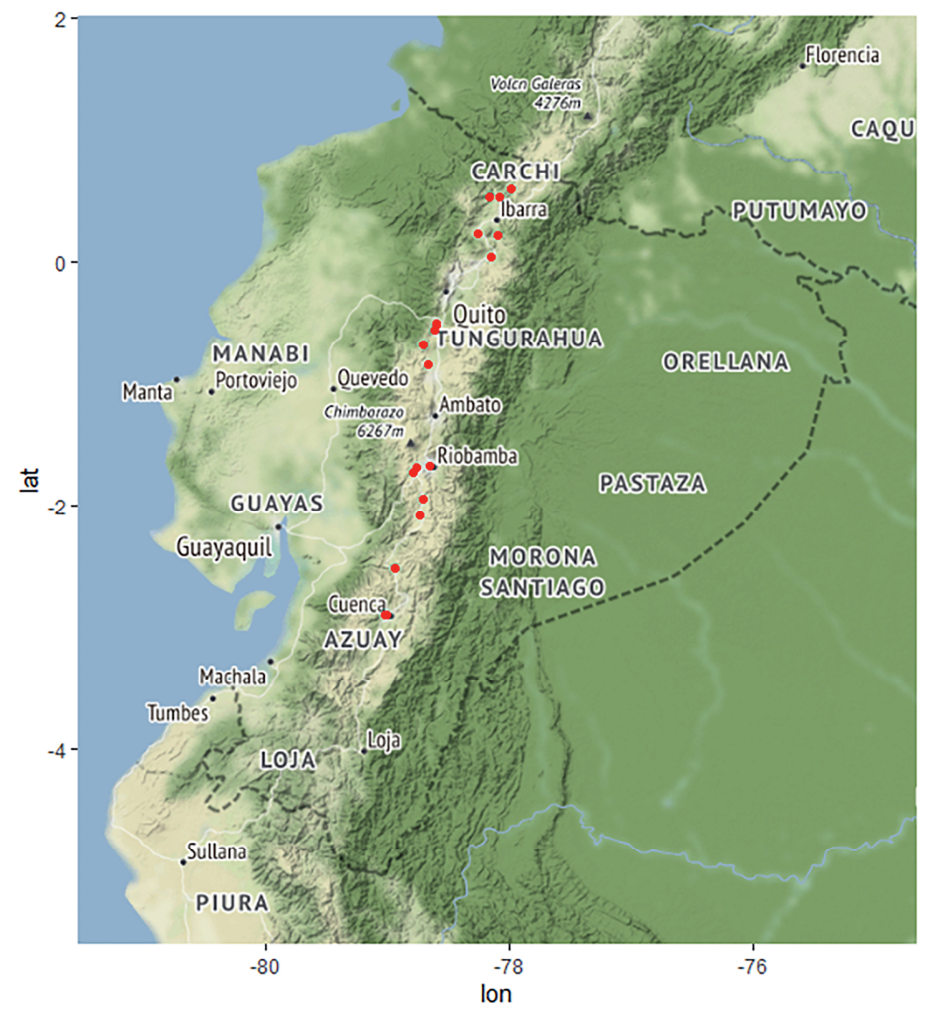

Figure 1. Geo-localization of the quinoa accessions collected in Ecuador. 
Table 1. Description of the genotypes screened in this study.

\begin{tabular}{|c|c|c|c|c|c|c|}
\hline ID cultivar & Origin* & $\begin{array}{l}\text { Altitude } \\
\text { (m.a.s.I) }\end{array}$ & Bitterness** & \begin{tabular}{|l|} 
Photoperiod \\
sensitivity***
\end{tabular} & Seed yield $\left(\mathrm{g} / \mathrm{m}^{2}\right)^{* * * *}$ & TSW (g)**** \\
\hline Pasto & Dutch variety, WUR & & Sweet & Daylenght neutral & 168 & 2.79 \\
\hline RedCarina & Dutch variety, WUR & & Very bitter & Daylenght neutral & 605 & 2.89 \\
\hline Tunkahuan & Ecuadorian variety, INIAP & 2815 & Sweet & Short day required & 677 & 2.97 \\
\hline 1_Carchi & ECU, Carchi, Espejo & 3021 & Sweet & Short day required & 769 & 3.22 \\
\hline 2_Carchi & ECU, Carchi, Mira & 2604 & Slightly bitter & Short day required & 938 & 2.88 \\
\hline 3_Carchi & ECU, Carchi, Espejo & 3024 & Sweet & Daylenght neutral & 728 & 4.38 \\
\hline 1_Imbabura & ECU, Imbabura, Angochagua & 2877 & Slightly bitter & Short day required & 842 & 3.05 \\
\hline 2_Imbabura & ECU, Imbabura, La Esperanza & 2708 & Slightly bitter & Short day required & 255 & 3.43 \\
\hline 3_Imbabura & ECU, Imbabura, Otavalo & 2530 & Slightly bitter & Short day required & 1127 & 2.84 \\
\hline 1_Pichincha & ECU, Pichincha, Mejia & 3265 & Bitter & Short day required & Didn't reach seed maturity & NA \\
\hline 2_Pichincha & ECU, Pichincha, Mejia & 3059 & Slightly bitter & Daylenght neutral & 699 & 3.8 \\
\hline 3_Pichincha & ECU, Pichincha, Aloasi & 3063 & Sweet & Short day required & 657 & 4.16 \\
\hline 1_Cotopaxi & ECU, Cotopaxi, Toacazo & 3311 & Slightly bitter & Short day required & 795 & 2.94 \\
\hline 2_Cotopaxi & ECU, Cotopaxi, Saquisili & 2924 & Bitter & Short day required & 972 & 3.13 \\
\hline 1_Chimborazo & ECU, Chimborazo, Colta & 3511 & Very bitter & Short day required & 825 & 2.82 \\
\hline 2_Chimborazo & ECU, Chimborazo, Guamote & 3080 & Very bitter & Short day required & Didn't reach seed maturity & NA \\
\hline 3_Chimborazo & ECU, Chimborazo, Guamote & 3305 & Very bitter & Short day required & 917 & 2.92 \\
\hline 4_Chimborazo & ECU, Chimborazo, Riobamba & 2756 & Slightly bitter & Short day required & 890 & 2.16 \\
\hline 5_Chimborazo & ECU, Chimborazo, Colta & 3165 & Bitter & Short day required & 1145 & 2.54 \\
\hline 1_Canar & ECU, Canar, Cachi & 2960 & Very bitter & Short day required & 853 & 2.47 \\
\hline 1_Azuay & ECU, Azuay, Cuenca, Museo Banco Central & 2590 & Very bitter & Short day required & 1246 & 3.82 \\
\hline 2_Azuay & ECU, Azuay, Cuenca, Mercado 10 de Agosto & 2561 & Bitter & Short day required & 802 & 4.16 \\
\hline 3_Azuay & ECU, Azuay, Cuenca, Mercado Feria Libre & 2590 & Bitter & Short day required & 625 & 2.84 \\
\hline 4_Azuay & ECU, Azuay, Cuenca, Mercado 3 de Noviembre & 2617 & Sweet & Short day required & 664 & 2.55 \\
\hline
\end{tabular}

* Origin: variety description or, Country, Province, Location

**Bitterness of the seeds was determined by foam test, based on the following height scale: sweet (foam height $<0.2 \mathrm{~cm}$ ), slightly bitter (foam height $0.2-2 \mathrm{~cm}$ ), bitter (foam height $2.5-4 \mathrm{~cm}$ ), very bitter (foam height $>4 \mathrm{~cm}$ )

***Based on the propagation of the seeds in long-day photoperiod

**** Yield traits based on cultivation in the Netherlands, with a simulated short day photoperiod ( $12 \mathrm{~h}$ light), greenhouse conditions, crop density, nonstress conditions

\subsection{Experimental conditions}

Plants were grown in crates $(40 \times 60 \mathrm{~cm})$ using potting soil as a substrate. A plant density of 100 plants $/ \mathrm{m}^{2}$ (24 plants per crate) was utilized to simulate crop density under commercial cultivation conditions. The growth of the 24 genotypes was evaluated under two salt concentrations (control and $250 \mathrm{mM} \mathrm{NaCl}$ ) in duplicates following a split plot design. The experiment was conducted in the greenhouse (Unifarm, Wageningen University \& Research, The Netherlands) between January and June 2019. A photoperiod of 12-hour light was imposed on the plants. The 12 hours of darkness were applied following sunset so the plants were exposed to a natural end-of-day signal. The greenhouse air humidity was set to a minimum of 80 $\%$. When the incoming shortwave radiation was below $200 \mathrm{Wm}^{-2}$, additional lighting was supplied $\left(100 \mathrm{Wm}^{-2}\right)$. The average day/night temperatures were set at $20^{\circ} \mathrm{C} /$ $12{ }^{\circ} \mathrm{C}$, respectively. The plants were irrigated with half-concentrated Hoagland's nutrient solution. Salt treatment started five weeks after sowing (2 weeks after transplanting). Salt was applied by incremental increases of $75 \mathrm{mM} \mathrm{NaCl}$ per day until the desired salt concentration was reached, and the soil salt concentration was monitored continuously by measuring the electrical conductivity in the leakage from 
the crates with a conductivity meter (Profline Cond 315i, Xylem Analytics, Germany). Irradiance, air temperature, water content and Electrical Conductivity (EC) in the crates was monitored with wireless sensors (Flower Power ${ }^{\mathrm{TM}}$ ).

\subsection{Assessment of growth and agronomical traits}

Plant height was measured weekly. Plant developmental stages were scored weekly according to a cardinal scale described elsewhere (Jaramillo Roman et al. 2020). Plants were harvested at maturity, 166 Days After Sowing (DAS). Seed yield and biomass of stems were measured per crate and reported as $\mathrm{g} / \mathrm{m}^{2}$. Thousand seed weight (TSW) was recorded using a seed counter (Contador, Pfeuffer $\mathrm{GmbH}$, Jefferson, OR, USA) and harvest index (HI) was calculated as the ratio of dry seed weight to dry stem biomass. The salt tolerance index (STI) was calculated as the ratio of seed yield of salt-treated plants to the seed yield of control $(0 \mathrm{mM} \mathrm{NaCl})$ plants.

\subsection{Assessment of physiological traits}

Several physiological traits were measured one, two and three months after the start of the salt treatment. Stomatal conductance $(g s)$ was measured on the abaxial side of the second fully developed non-shadowed leaf using a portable leaf porometer (Decagon Devices Inc., WA, Australia) between 9:00 and 15:00 hours on a sunny day. Relative water content (RWC) was calculated as $R W C=\frac{(F W-D W)}{(T W-D W)}$ * $100 \%$, were $\mathrm{FW}$ is the fresh weight, DW is the dry weight and TW is the turgid weight of an entire single young leaf. Turgid weight was determined after the leaf was imbibed in ultrapure water (Milli-Q®) in the dark for 12 hours. The RWC of each experimental unit was evaluated in triplicate. From the same young leaves, the specific leaf area (SLA) was calculated as the amount of leaf area per unit of dry weight $\left(\mathrm{cm}^{2} / \mathrm{g}\right)$. The ion contents in young leaves were measured using lon Chromatography as described in (Jaramillo Roman et al. 2020). Briefly, $25 \mathrm{mg}$ of dry leaves was ashed in a furnace at $550{ }^{\circ} \mathrm{C}$ for $5 \mathrm{~h}$. Ash was dissolved in $1 \mathrm{~mL} 3 \mathrm{M}$ formic acid at constant shaking of $500 \mathrm{rpm}$ for $15 \mathrm{~min}$ at $99^{\circ} \mathrm{C}$. The dissolved ashes were $400 \times$ diluted with Milli-Q尺 water and injected to the IC column. Ion contents

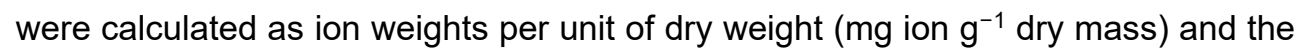
ion concentrations were estimated considering the water content of the tissue based on the difference between fresh and dry weights. The ratio $\mathrm{K}^{+} / \mathrm{Na}^{+}$was calculated as $\mathrm{mg} \mathrm{K}^{+} / \mathrm{mg} \mathrm{Na}^{+}$content. 


\subsection{Afrosimetric estimation of the saponin content in seeds}

The saponin content in quinoa seeds was estimated using a standard afrosimetric method as described in (Koziol 1991). In brief, $500 \mathrm{mg}$ of seeds were weighed in a $10 \mathrm{~mL}$ Falcon tube ${ }^{\circledR}$. After adding $5 \mathrm{~mL}$ of milli-q water, the tube was vigorously shaken for $2 \mathrm{~min}$. The height of the foam layer was measured 5-10 s after shaking. The seeds of the bitter variety Red Carina were used for an internal calibration of the test. The bitterness of the seeds was determined based on the following scale: sweet (foam height $<0.2 \mathrm{~cm}$ ), slightly bitter (foam height $0.2-2 \mathrm{~cm}$ ), bitter (foam height 2- $4 \mathrm{~cm}$ ), and very bitter (foam height $>4 \mathrm{~cm}$ ) (Mastebroek et al. 2000).

\subsection{Data analysis}

General analyses of variance (ANOVA) were performed to determine the significance of genotypic differences, salt treatment differences and their interactions $(p<0.05)$. The analyses were performed following a standard procedure for a linear mixed model, for which genotype and salt treatment were considered fixed effects and blocks random effects. Multiple comparison analyses were performed using Fisher's protected least significant difference (LSD) test on genotype means. Interrelationships between agronomical and physiological traits were analysed by means of Pearson correlations. All statistical analyses were performed using the software Genstat 19th Edition (VSN International Hemel Hempstead, UK). Principal component analysis (PCA) was used as a multivariate analysis tool to understand the interrelationship between genotypes and traits. PCAs were performed using the prcomp() function in $R$ ( $R$ 2019). A mantel test was run to compare a genetic distance matrix based on 15 SSR markers described in our previous publication (Salazar et al. 2019), and a Euclidean matrix based on the agronomical traits measured in this study, between all pairs of genotypes. The mantel test was performed using the mantel.randtest() function of the $\mathrm{R}$ package adegenet (Jombart 2008). 


\section{Results}

\subsection{Ecuadorian quinoa displayed large variation for morphological traits and salt tolerance}

The 22 Ecuadorian genotypes selected in this study displayed high diversity for agronomical traits. The seed yield in control conditions ranged from 255 to 1245 $\mathrm{g} / \mathrm{m}^{2}$ (average $820 \mathrm{~g} / \mathrm{m}^{2}$ ) (Figure 2A). Two genotypes (1-Pichincha and 2Chimborazo) did not reach seed maturity during the time of the experiment. These genotypes were the last to flower, and at the end of the experiment they were still producing pollen. In addition, they had the highest stem biomass and height, which suggests that vegetative growth in these two lines continued until the end of the trial. Possibly, the artificial 12 hrs dark-light cycle in the greenhouse did not properly induce the switch from the vegetative to the reproductive stage. The dry biomass of the stems of the 22 genotypes ranged from $350 \mathrm{~g} / \mathrm{m}^{2}$ to $1810 \mathrm{~g} / \mathrm{m}^{2}$ with an average of $804 \mathrm{~g} / \mathrm{m}^{2}$ (Figure 2B). A strong negative correlation $(-0.91, p<0.001)$ was observed between the stem biomass and the harvest index (in this study defined as the ratio between seed yield and stem biomass), and no correlation was found between seed yield and harvest index. On average, the seed biomass was $56 \%$ of the stem biomass (Figure $2 \mathrm{C}$ ). Large variation was also observed for the thousand seed weight, ranging from 2.4 to $4.4 \mathrm{~g}$ (Figure 2D). This is one of the prioritized traits in quinoa breeding programs. The large variation and big seed size observed in some of the genotypes of this study indicate the potential of this collection and Ecuadorian germplasm for future breeding purposes. 

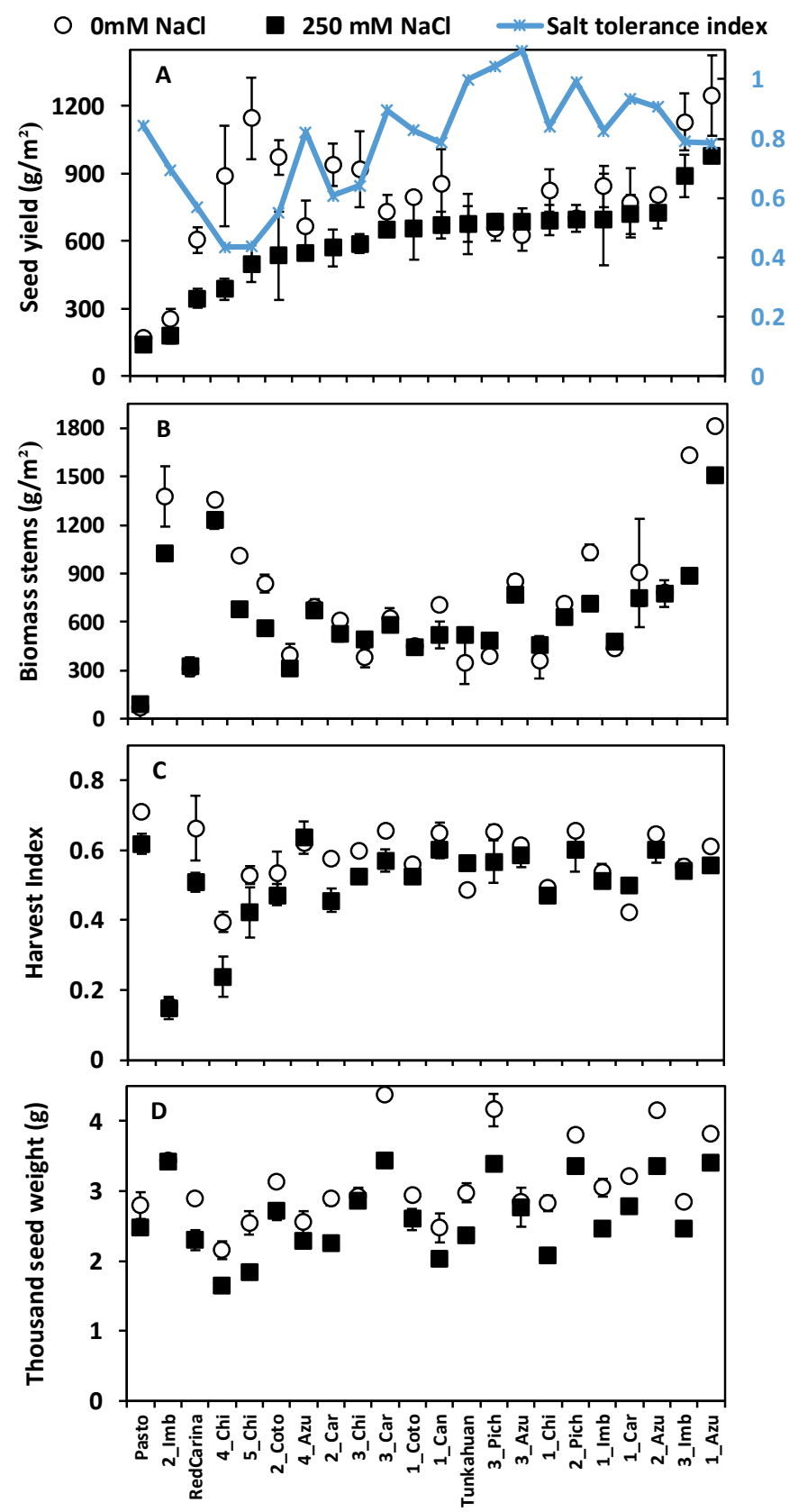

Figure 2. Agronomical traits of quinoa accessions growing under control and salt stress conditions (250 $\mathrm{mM} \mathrm{NaCl}$ ). A) Seed yield B) Stems biomass C) Harvest index (defined as the ratio of seeds to stem biomass) D) Thousand seed weight. Means of 2 replicates. Error bars indicate SE of individual means. 
The response to salinity of the assessed genotypes was in line with the high salt tolerance that characterizes quinoa (Adolf et al. 2013; Hinojosa et al. 2018). Despite the high salinity applied $(250 \mathrm{mM} \mathrm{NaCl}, 25 \mathrm{dS} / \mathrm{m}$, equivalent with half strength seawater) the average yield reduction was only $22 \%$. Out of the 20 Ecuadorian genotypes that produced seeds, 10 had a reduction in yield of $20 \%$ or less, and the yield of Tunkahuan, 3-Pichincha, 3-Azuay and 2-Pichincha was even not significantly affected by the salt treatment. Others genotypes were less tolerant to salinity, with the highest yield reduction of $56 \%$ observed for 4- Chimborazo and 5- Chimborazo (Figure 2A). The maximum reduction in stem biomass was $33 \%$ in the genotypes 2-Cotopaxi and 5-Chimborazo while 6 genotypes showed no significant differences between the control and salt treated plants (Figure 2B). Thousand seed weight was reduced by $15 \%$ on average. Only in 3 genotypes (1Azuay, 3-Chimborazo and 3-Azuay), this trait was not affected by salinity (Figure 2D). Significant variation was observed for height at the end of the season, both between cultivars and in the effect of salt stress (Figure 3 and Supplementary Table 1). On average, the height of the Ecuadorian genotypes was $170 \mathrm{~cm}$ under control conditions, and the average reduction caused by the salt treatment was $20 \%$. Height significantly correlated to stem biomass, both in control $(r=0.92)$ and stress conditions ( $r=0.7)$ (Figure 4).

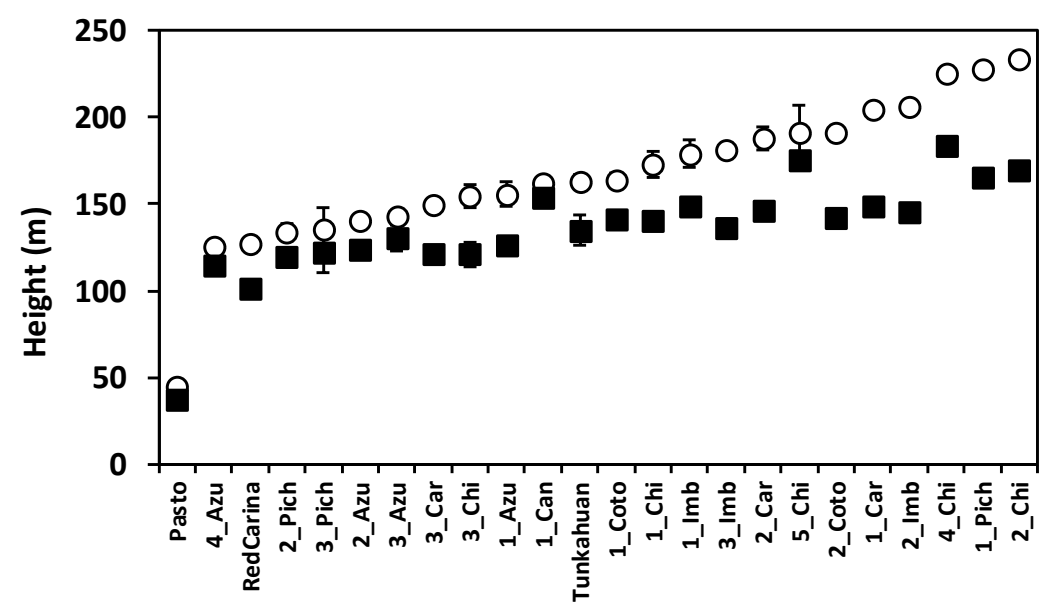

Figure 3. Height of quinoa accessions growing under control and salt stress conditions ( $250 \mathrm{mM} \mathrm{NaCl})$. Means of 2 replicates. Error bars indicate SE of individual means. 


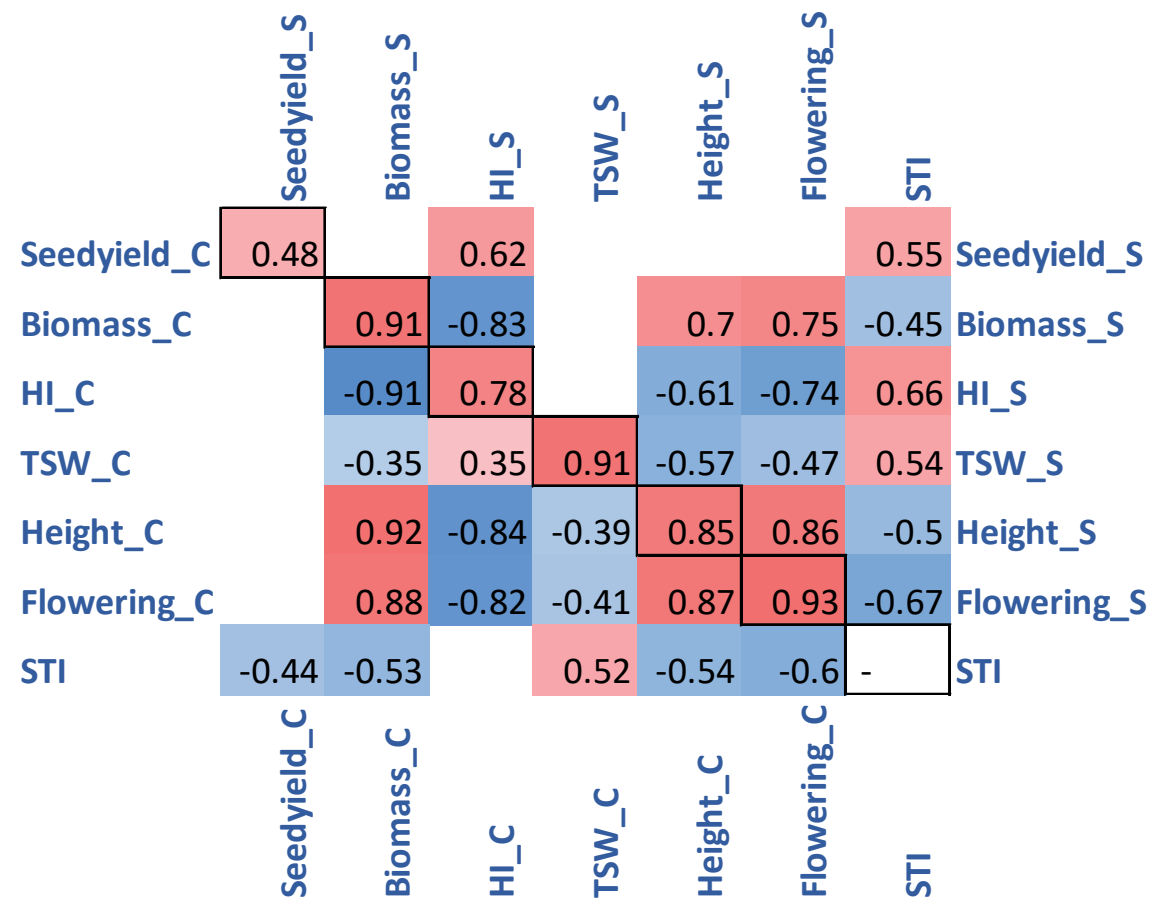

Figure 4. Correlations -of genotypic means- between the agronomical traits evaluated in this study. Only significant Pearson correlations are shown $(p<0.001)$. Red values indicate positive correlation coefficients and blue values indicate negative correlation coefficients. Coefficients in the diagonal indicate the relation of the same trait between control and stress conditions. Below the diagonal the correlations between traits under control conditions are given and above the diagonal the correlations under stress conditions.

The development of the genotypes was monitored throughout the season. On average, the Ecuadorian genotypes started flowering 77 days after sowing (DAS). Early genotypes flowered around 58 DAS and late genotypes required up to 96 days. The two genotypes that did not reach seed maturity in the course of the experiment (1-Pichincha and 2-Chimborazo) were the last to flower (96 DAS). In most of the genotypes, flowering time was delayed by the salt treatment, while other developmental phases such as seed filling and seed maturity were not influenced by salt, resulting in shorter time between flowering and maturation (Table 2). Both in control and stress conditions, flowering time (in days after sowing) positively correlated with stem biomass and negatively correlated with Harvest Index, indicating that the longer it takes to switch between the vegetative and reproductive phase, the less assimilates were allocated to seeds (Figure 4). 
Table 2. Developmental stages of cultivars under control and stress conditions. Values represent days after sowing (DAS). Three main physiological stages are presented (days to flowering, days to seed filling and days to seed maturity).

\begin{tabular}{l|c|c|c|c|c|c} 
Cultivar & \multicolumn{2}{c}{ Flowering (DAS) } & \multicolumn{2}{c}{ Seed filling (DAS) } & \multicolumn{2}{c}{ Seed maturity (DAS) } \\
\hline & Control & Stress & Control & Stress & Control & Stress \\
\hline Pasto & 54 & 49 & 75 & 75 & 96 & 96 \\
3_Azuay & 58 & 58 & 90 & 90 & 138 & 138 \\
3_Carchi & 61 & 61 & 96 & 96 & 138 & 125 \\
2_Pichincha & 61 & 61 & 90 & 90 & 125 & 125 \\
3_Pichincha & $\mathbf{6 1}$ & $\mathbf{6 5}$ & 96 & 96 & 138 & 138 \\
4_Azuay & 61 & 61 & 90 & 90 & 138 & 138 \\
RedCarina & 65 & 61 & 90 & 90 & 110 & 110 \\
2_Azuay & $\mathbf{6 1}$ & $\mathbf{6 4}$ & 110 & 110 & 166 & 138 \\
1_Chimborazo & $\mathbf{7 5}$ & $\mathbf{8 2}$ & 110 & 110 & 166 & 166 \\
3_Chimborazo & $\mathbf{7 5}$ & $\mathbf{8 2}$ & 110 & 110 & 166 & 166 \\
1_Canar & $\mathbf{7 5}$ & $\mathbf{8 2}$ & 110 & 110 & 166 & 166 \\
3_Imbabura & $\mathbf{7 5}$ & $\mathbf{8 2}$ & 110 & 110 & 166 & 166 \\
1_Imbabura & $\mathbf{7 8}$ & $\mathbf{9 0}$ & $\mathbf{1 0 5}$ & $\mathbf{1 1 0}$ & 166 & 166 \\
1_Carchi & $\mathbf{8 4}$ & $\mathbf{9 0}$ & $\mathbf{1 0 0}$ & $\mathbf{1 0 5}$ & 166 & 166 \\
Tunkahuan & $\mathbf{8 2}$ & $\mathbf{8 2}$ & 110 & 110 & 166 & 166 \\
1_Cotopaxi & $\mathbf{8 2}$ & $\mathbf{8 4}$ & 110 & 110 & 166 & 166 \\
1_Azuay & $\mathbf{8 2}$ & $\mathbf{9 0}$ & 110 & 110 & 166 & 166 \\
2_Carchi & $\mathbf{8 4}$ & $\mathbf{8 8}$ & 110 & 110 & 166 & 166 \\
2_Cotopaxi & $\mathbf{8 4}$ & $\mathbf{9 1}$ & 110 & 110 & 166 & 166 \\
5_Chimborazo & $\mathbf{9 0}$ & $\mathbf{1 1 0}$ & 120 & 120 & 166 & 166 \\
1_Pichincha & $\mathbf{9 0}$ & $\mathbf{9 6}$ & 138 & 130 & $*$ & $*$ \\
4_Chimborazo & $\mathbf{9 0}$ & $\mathbf{1 1 0}$ & 138 & 138 & 166 & 166 \\
2_Imbabura & $\mathbf{9 6}$ & $\mathbf{9 3}$ & 120 & 120 & 166 & 166 \\
2_Chimborazo & $\mathbf{9 6}$ & $\mathbf{1 3 6}$ & 138 & $*$ & $*$ & $*$
\end{tabular}

*Seed maturity was not reached within the timeframe of the experiment 
Figure 5 depicts the seed yield of the evaluated genotypes under control and stress conditions. Genotypes were classified in four groups; those with higher yield than the average of all genotypes under stress as well as control conditions (green in Figure 5), genotypes that yield less than average under both conditions (grey), genotypes that yield higher than average only under control conditions (red) or only under stress conditions (blue). Several genotypes displayed GxE interaction, like 4_Chimborazo and 5_Chimborazo. Under control conditions, these genotypes are high-yielding lines with an average seed yield of 890 and $1145 \mathrm{~g} / \mathrm{m}^{2}$ respectively, while under stress conditions, their yields were reduced by $56 \%$ to 386 and 499 $\mathrm{g} / \mathrm{m}^{2}$, respectively.

Two Dutch varieties (Pasto and Red Carina) were included in this study for further comparison. Seed yields of these varieties in control conditions were 168 and $605 \mathrm{~g} / \mathrm{m}^{2}$ for Pasto and Red Carina, respectively. Under salt stress, the yield of Pasto was reduced by $15 \%$ and the yield of Red Carina by $43 \%$.

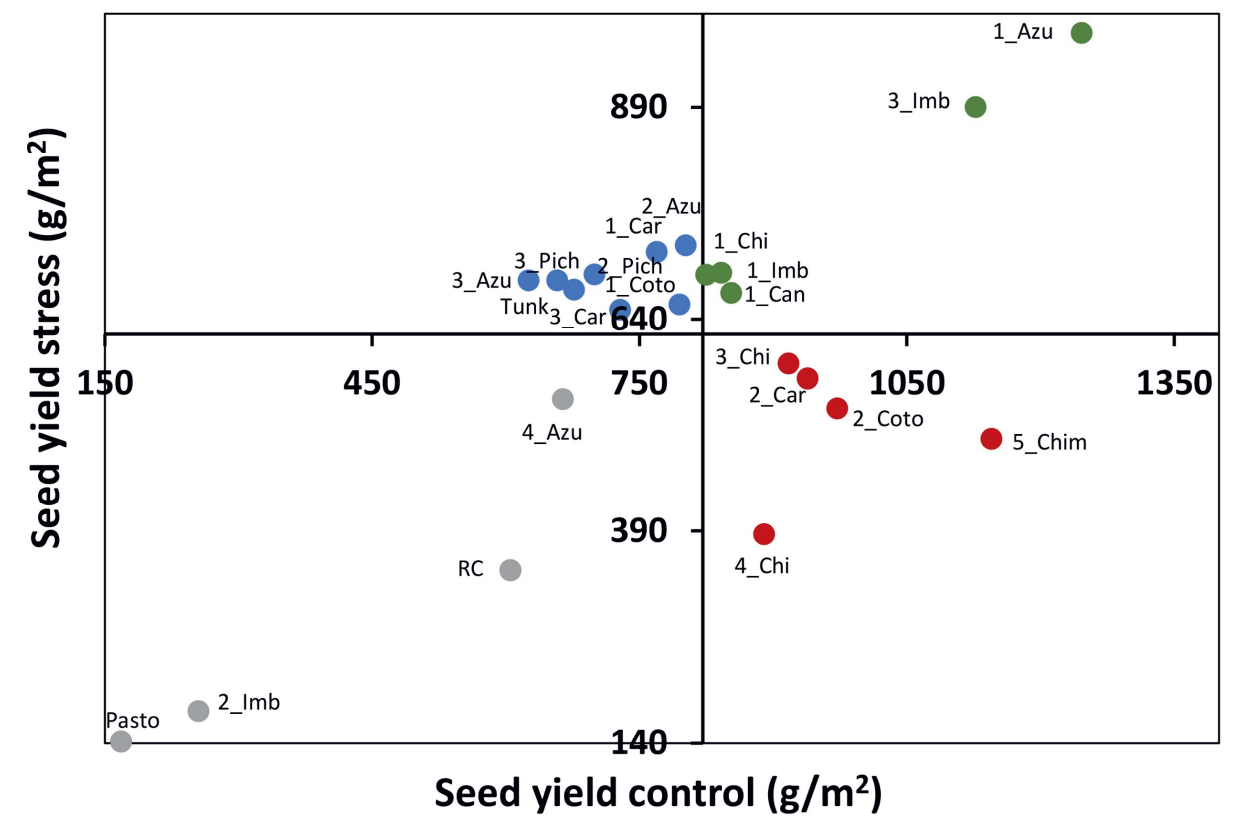

Figure 5. Overall classification of genotypes based on their yield in control and stress conditions. The $x$ axis is set to the average seed yield under control conditions and $y$-axis to the average seed yield under stress conditions. Genotypes that yield better than average in both conditions are presented in green, those that yield less than average in both conditions in grey, those than perform better than the average just in control conditions are shown in red and just in stress conditions in blue. 


\subsection{Genetic diversity on the ion dynamics of Ecuadorian quinoa}

The concentrations of $\mathrm{Na}^{+}, \mathrm{K}^{+}, \mathrm{Ca}^{2+}, \mathrm{Mg}^{2+}$, and $\mathrm{Cl}^{-}$ions were measured in young leaves of the 24 genotypes after one, two and three months of salt stress application.

After one month of stress, all cation concentrations were higher in the leaves of plants growing under stress conditions compared to control (Figure 6 and Supplementary Figure 1). The average $\left[\mathrm{Na}^{+}\right]$in young leaves after one and two months of salt stress was $70 \mathrm{mM}$ (Figure 6A), which is considerably lower than the $250 \mathrm{mM} \mathrm{NaCl}$ in the root media and indicates that quinoa plants were very well able to exclude $\mathrm{Na}^{+}$from young leaves. Towards the end of the season (three months after start of stress application) the levels of $\mathrm{Na}^{+}$in young leaves increased substantially reaching an average concentration of $203 \mathrm{mM}$. While the $\left[\mathrm{Na}^{+}\right]$in young leaves remained below the $250 \mathrm{mM} \mathrm{NaCl}$ in the root media for most of the genotypes, concentrations exceeding $400 \mathrm{mM}$ were measured in some genotypes including 2Pichincha, 1-Azuay and Red Carina. Genotypic differences in the $\mathrm{Na}^{+}$accumulation in young leaves were also observed throughout the season (Supplementary Figure $1 \mathrm{~A}-\mathrm{C}$ ). For some genotypes, like Pasto and 3-Azuay, the concentration of $\mathrm{Na}^{+}$was not significantly different between treatments, and $\left[\mathrm{Na}^{+}\right]$was lower than $50 \mathrm{mM}$ after 1 month of stress. Pasto had the lowest ion accumulation after one month of stress, and this increased to $104 \mathrm{mM}$ after 2 months and to $253 \mathrm{mM}$ at the latest time point. Other genotypes had relatively high levels of $\mathrm{Na}^{+}$at all-time points, like 1_Azuay and 2_Pichincha, although only after three months of salt stress the concentration was higher than in the root medium. The leaf $\left[\mathrm{Na}^{+}\right]$at the third sampling point had a positive significant correlation with seed yield and TSW under stress conditions (Figure 7).

In line with previous reports using different quinoa germplasm, $\left[\mathrm{K}^{+}\right]$significantly increased in young leaves of Ecuadorian quinoa plants growing under salt stress compared to control (Hariadi et al. 2011; Jaramillo Roman et al. 2020). After 1 month of salt stress, all the genotypes had a higher $\left[\mathrm{K}^{+}\right]$under salt stress compared to control. The average $\left[\mathrm{K}^{+}\right]$was $357 \mathrm{mM}$ under salt stress compared to $267 \mathrm{mM}$ under control conditions, with a further increase after two months of stress to $458 \mathrm{mM}$ under stress compared to $339 \mathrm{mM}$ under control conditions. After three months of treatment, the average $\left[\mathrm{K}^{+}\right]$decreased to $397 \mathrm{mM}$ under stress conditions. For most of the genotypes, the $\left[\mathrm{K}^{+}\right]$was higher under stress conditions, while for some genotypes there was no significant difference in the concentration between the two treatments (Supplementary Figure $1 \mathrm{D}-\mathrm{F}$ ). The $\left[\mathrm{K}^{+}\right]$at all-time points had a significant negative correlation with stem biomass and height (Figure 7). 
The average $\mathrm{K}^{+} / \mathrm{Na}^{+}$ratio was 13.7 in control conditions and it decreased to 8.9 in stress conditions one month after the stress treatment was started, but after two months, the average $\mathrm{K}^{+} / \mathrm{Na}^{+}$ratio was restored to 11.5 in the salt-stressed plants. For most genotypes, no significant difference was found between treatments at this stage. However, at the last sampling point, the average $\mathrm{K}^{+} / \mathrm{Na}^{+}$ratio decreased considerably to 5 under stress conditions (Figure 6D). The ratio was more influenced by the capacity of quinoa to exclude or accumulate $\mathrm{Na}^{+}$in leaves than the retention of $\mathrm{K}^{+}$, with a strong negative correlation with leaf $\left[\mathrm{Na}^{+}\right]$in all harvests (Figure 7).

The concentration of $\mathrm{Mg}^{2+}$ was hardly affected by salt during the two first months of salt application, but it dropped significantly from $170 \mathrm{mM}$ under control conditions to $90 \mathrm{mM}$ under stress conditions after three months of the application of the salt treatment (Figure $6 \mathrm{E}$ ). During the first sampling point, $\left[\mathrm{Ca}^{2+}\right]$ was increased in the salt treatment compared to the control, but it was significantly reduced for all genotypes after two and three months of the start of salt treatment. At the last sampling point, the average concentration was $88 \mathrm{mM}$ under control conditions compared to $26 \mathrm{mM}$ under stress conditions (Figure 6F). However, based on [ $\left.\mathrm{Ca}^{2+}\right]$ values previously reported for plants, these genotypes are very likely not suffering from $\mathrm{Ca}^{2+}$ deficiency under these conditions (Genc et al. 2010).

$\mathrm{Cl}^{-}$progressively accumulated to higher levels than $\mathrm{Na}^{+}$in young leaves. After one month of salt stress, the average $\left[\mathrm{Cl}^{-}\right]$in young leaves was $176 \mathrm{mM}$ in plants exposed to the salt stress compared to $9 \mathrm{mM}$ in control plants. After two months of salt stress, $\left[\mathrm{Cl}^{-}\right]$further increased to $246 \mathrm{mM}$, equalizing the $\left[\mathrm{Cl}^{-}\right]$in the root medium, and after three months of salt stress the average $\left[\mathrm{Cl}^{-}\right]$was $315 \mathrm{mM}$ (Figure $6 \mathrm{C}$ ), with variation between the varieties (Supplementary Figure $1 \mathrm{G}-\mathrm{I}$ ). $\left[\mathrm{Cl}^{-}\right]$had a significant negative correlation with most of the agronomical traits (seed yield, stem biomass, and plant height), a positive correlation with the SLA and $\left[\mathrm{Na}^{+}\right]$, and a negative correlation with the $\left[\mathrm{K}^{+}\right]$after two and three months of stress (Figure 7). 


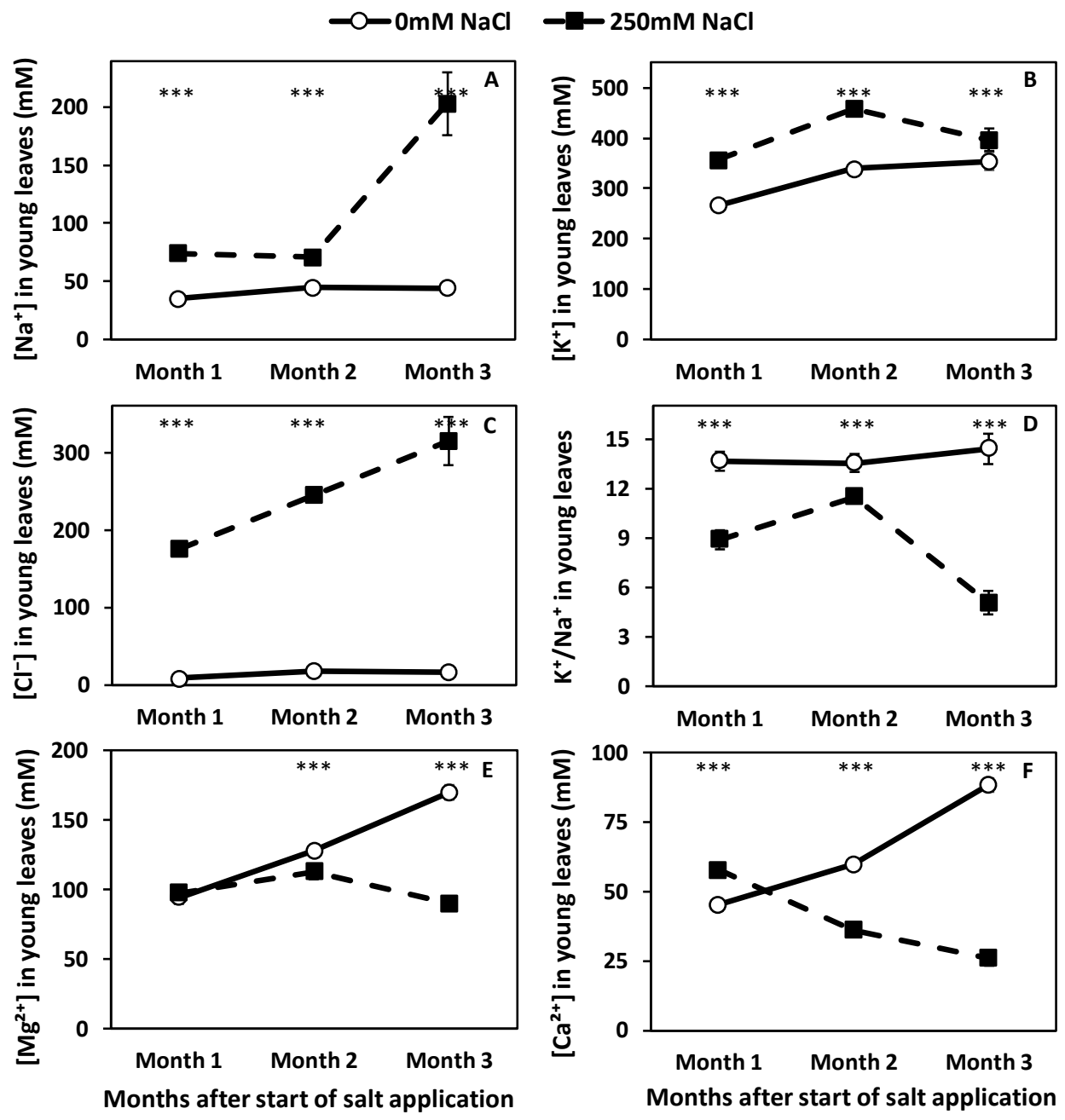

Figure 6. Ion contents in young leaves measured after one, two and three months of the start of the salt treatment. Means of the 24 genotypes are presented. Error bars indicate SE of individual means. Asterisks denote statistically significant differences $\left({ }^{*} p \leq 0.05,{ }^{* *} p \leq 0.01,{ }^{* *} p \leq 0.001\right)$ between the salt treatment and the control. 


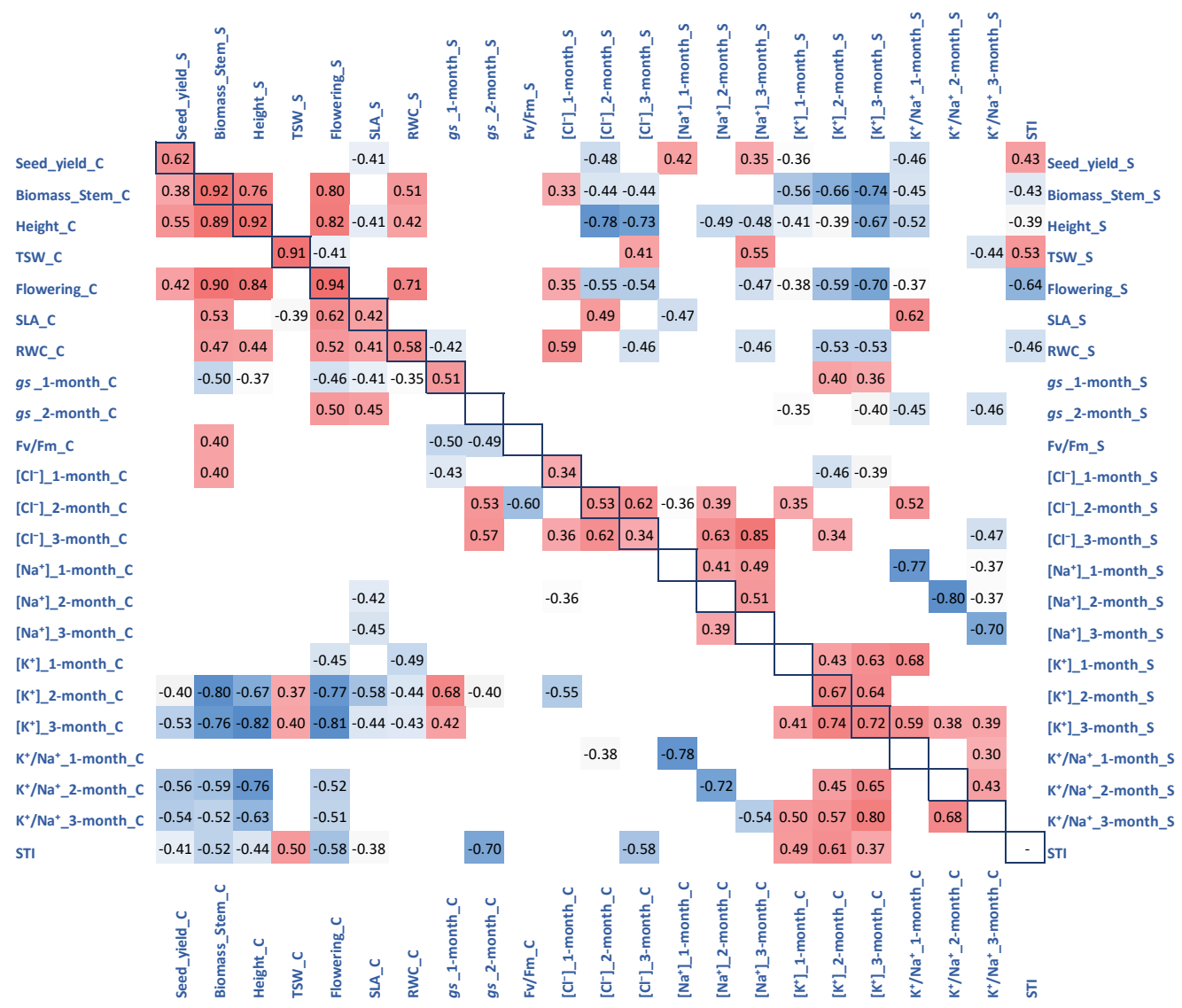

Figure 7. Correlations -of genotypic means- among agronomical and physiological traits in control and stress conditions. Only Pearson correlation coefficients that differed significantly from zero $(p<0.001)$ are presented. Values in blue indicate a negative correlation and in red a positive correlation between traits. Coefficients in the diagonal indicate the relation of the same trait between control and stress conditions. Below the diagonal the correlations between traits under control conditions are given and above the diagonal the correlations under stress conditions. 


\subsection{Physiological traits in response to salt stress}

The effect of the salt treatment on stomatal conductance ( $g s$ ) was significant only after several weeks of salt stress, in line with what we previously reported (Jaramillo Roman et al. 2020). The effect of salinity on stomatal conductance was barely significant after one month, with an average conductance of $105 \mathrm{mmol} / \mathrm{m}^{2} \mathrm{~s}$ for plants in control conditions and $86 \mathrm{mmol} / \mathrm{m}^{2} \mathrm{~s}$ for stressed plants (Figure $8 \mathrm{~A}$ ). No significant differences were found between genotypes or for the salt stress $\mathrm{x}$ genotype interaction (Supplementary Table 1). After two months of stress, the average reduction in stomatal conductance due to the salt treatment was $30 \%$. For some genotypes the reduction due to salinity was as much as $50 \%$ (5-Chimborazo), while other genotypes had a mild response with less than $20 \%$ reduction (Figure $8 \mathrm{~B})$. The decrease in stomatal conductance appeared to be linked to the salt tolerance of the analysed genotypes: it had a negative correlation with seed yield $(r=-0.46), S T I(r=-0.61)$ and a positive correlation with $\mathrm{Cl}^{-}$accumulation in young leaves $(r=0.32)$, SLA $(r=0.55)$ and RWC ( $r=0.42)$ (Figure 7).

Relative water content was measured in young leaves after 2 months of salt treatment (Figure 9A). For most of the genotypes, RWC was not significantly different between stress and control. On average, the leaf RWC decreased slightly from 77 $\%$ in control conditions to $73 \%$ under stress. No significant differences were found for the Specific Leaf Area (SLA) between genotypes or salt treatments (Figure 9B). 

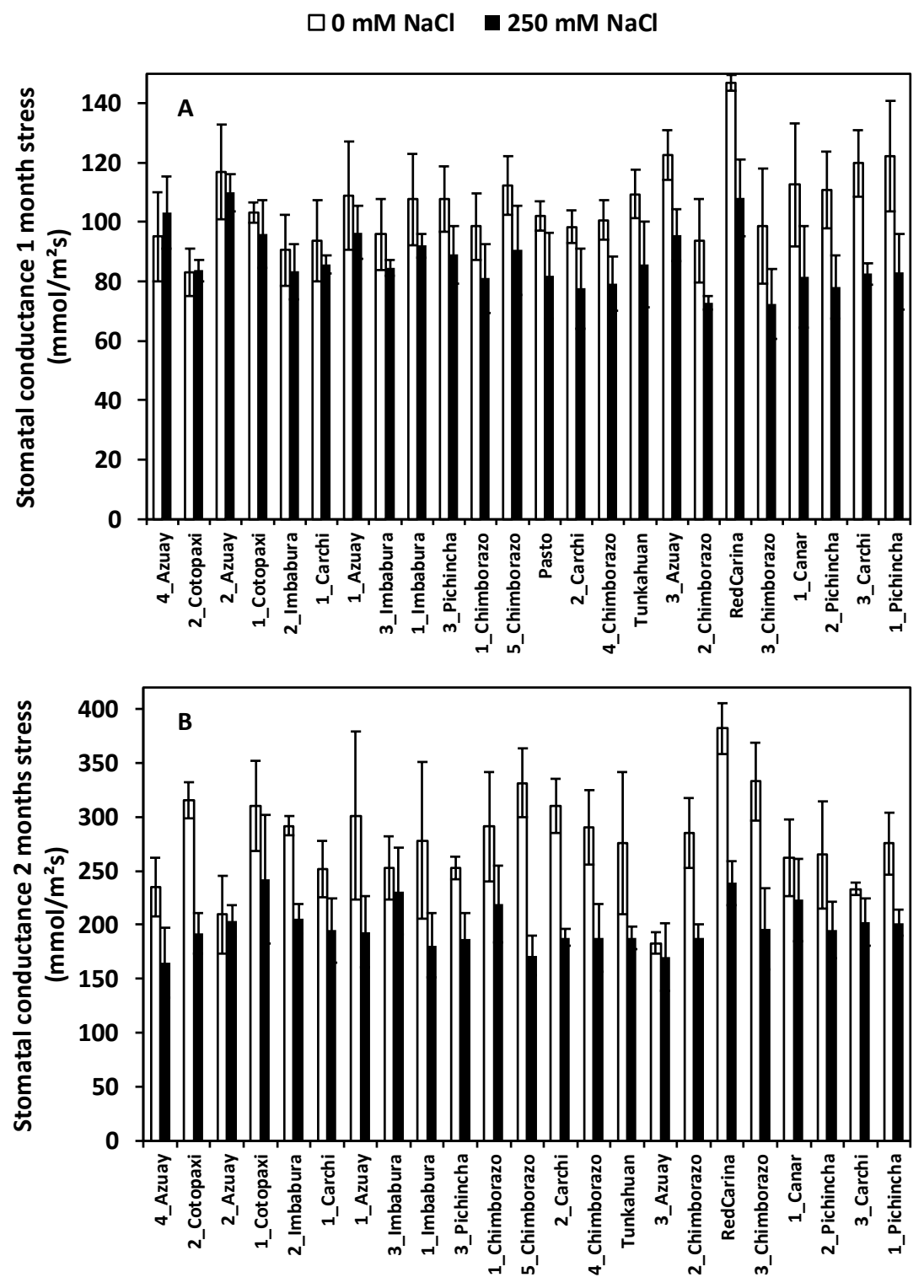

Figure 8. Stomatal conductance ( $g s)$ of quinoa accessions growing under control and salt stress conditions $(250 \mathrm{mM} \mathrm{NaCl})$. A) Stomatal conductance one month after the start of salt stress; B) Stomatal conductance two months after the start of salt stress. Means of two plots per genotype and two measurements per plot. Error bars indicate SE of individual means. 

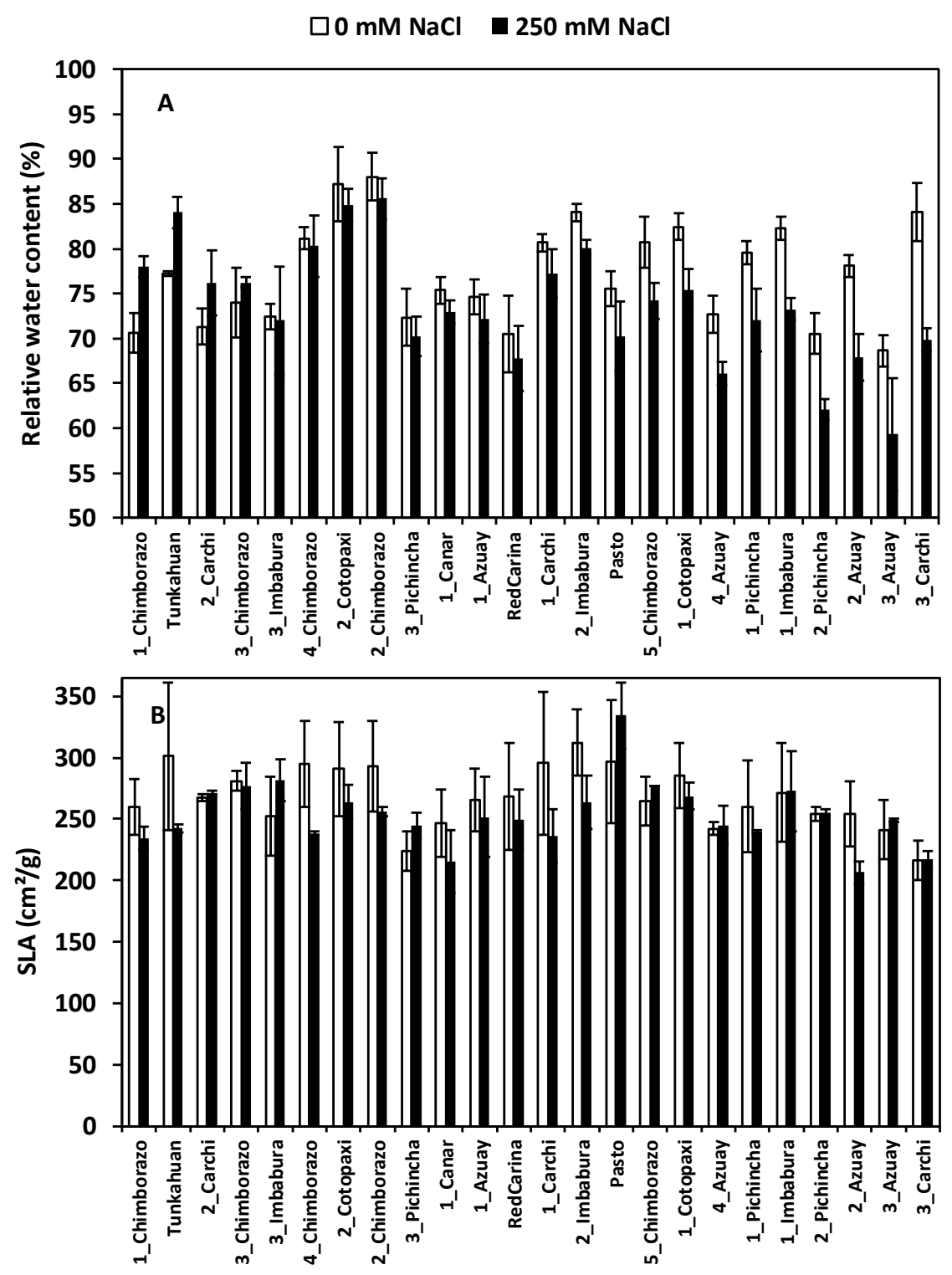

Figure 9. Physiological traits of quinoa accessions growing under control and salt stress conditions (250 $\mathrm{mM} \mathrm{NaCl}$ ). A) Relative water content two months after the start of salt stress B) Specific leaf area two months after the start of salt stress. Means of two plots per genotype and two measurements per plot. Error bars indicate SE of individual means. 


\subsection{Genetic diversity of Ecuadorian quinoa}

The genetic diversity of the collection was further assessed by identifying the traits that contribute most to the salt tolerance variation and examining their relationships with a principal component analysis (PCA). The following variables were included: STI based on seed yield, $\left[\mathrm{Cl}^{-}\right],\left[\mathrm{Na}^{+}\right],\left[\mathrm{K}^{+}\right]$and the ratio $\mathrm{K}^{+} / \mathrm{Na}^{+}$in young leaves of stressed plants after one and two months of salt stress treatment, salinity-induced decrease in gs after one and two months of the start of the salt treatment, RWC and SLA in young leaves of salt stressed plants (Figure 10). The first four principal components accounted for $76 \%$ of the variability amongst the 20 Ecuadorian lines that produced seeds under control as well as stress conditions, and had eigenvalues $>1.5$ (Supplementary Table 2). The first principal component accounted for $27.4 \%$ of the total variation. The variables that contributed most to $\mathrm{PC} 1$ were $\left[\mathrm{K}^{+}\right]$and $\mathrm{K}^{+} / \mathrm{Na}^{+}$ratio at the two sampling points and these were positively correlated with STI. The genotypes on the right side of the PCA had a high $\left[\mathrm{K}^{+}\right]$in young leaves, high $\mathrm{K}^{+} / \mathrm{Na}^{+}$ratio and high salt tolerance (seed yield reduction $<20$ $\%)$. A second group of genotypes on the left side of the plot were grouped based on high $\left[\mathrm{Cl}^{-}\right]$during the first month of stress and high RWC supported by a high decrease in the stomatal conductance. These genotypes had a lower STI (more than $40 \%$ yield reduction) (2_Cotopaxi, 2_Imbabura, 2_Carchi, 3_Chimborazo and 5_Chimborazo). The second PC accounted for $23.1 \%$ of the total variation. The main variables that contributed to this $\mathrm{PC}$ were the $\left[\mathrm{Na}^{+}\right]$at both sampling points, and in the opposite direction the $\mathrm{K}^{+} / \mathrm{Na}^{+}$ratio. This $\mathrm{PC}$ clustered the genotypes with higher accumulation of $\mathrm{Na}^{+}$(1_Canar, 1_Carchi, 1_Imbabura, 4_Chimborazo, 1_Chimborazo), with only a limited contribution to the STI of the genotypes.

A Mantel-test comparing the genetic diversity of our collection of Ecuadorian quinoa based on SSR markers as presented previously (Salazar et al. 2019) and some of the phenotypic traits evaluated in this study indicated a low and not significant correlation between both matrices $(r=0.14, p=0.81)$. This is an indication that the measured traits were not confined to specific genetic material clustered in a specific phylogenetic clade. A larger set of genotypes and markers would be required to understand the genetic diversity of this collection. 


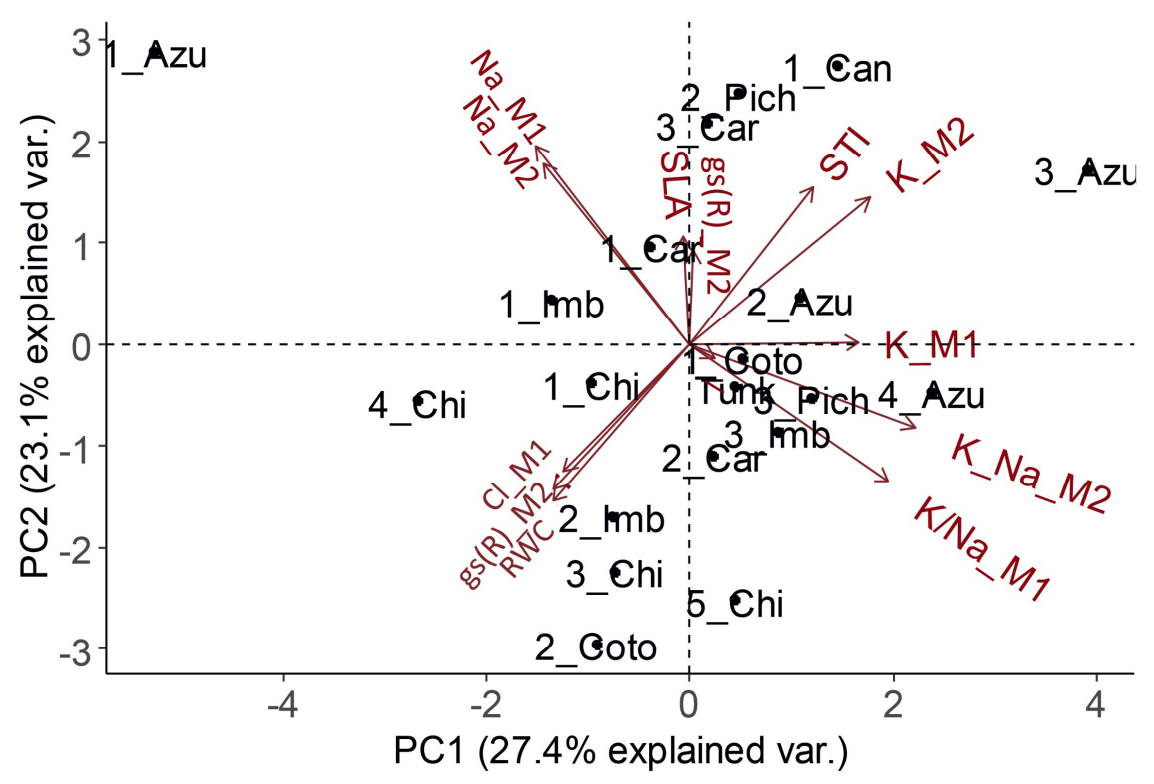

Figure 10. Principal component biplot displaying the variation in the collection of Ecuadorian quinoa genotypes. Genotypes are indicated as black dots and traits as red vectors. Abbreviations: Na_M1/ $\mathrm{Na} \_\mathrm{M} 2\left(\left[\mathrm{Na}^{+}\right]\right.$: young leaves stressed plants after 1 month/2 months treatment); K_M1/ K_M2 ([K+ $]$ : young leaves stressed plants after 1 month/2 months treatment); Cl_M1/ Cl_M2 ([Cl-]: young leaves stressed plants after 1 month/2 months treatment); $\mathrm{K} \_\mathrm{Na} \_\mathrm{M} 1 / \mathrm{K} \_\mathrm{Na} \_\mathrm{M} 2\left(\mathrm{~K}^{+} / \mathrm{Na}^{+}\right.$: young leaves stressed plants after 1 month/2 months treatment); SLA: specific leaf area stressed plants; STI: salt tolerance index based on seed yield); $g s(\mathrm{R}) \_\mathrm{M} 1 / \mathrm{gs}(\mathrm{R}) \_\mathrm{M} 2$ : reduction of $g s$ due to salinity after 1 month/ 2 months treatment. 


\section{Discussion}

Quinoa is known to be a highly salt tolerant crop, and is considered a facultative halophyte due to its ability to grow optimally in normal cultivation conditions, but also tolerate high salt levels well and even survive at salinity levels above that of seawater. A considerable amount of literature is available on salt tolerance of quinoa (Hinojosa et al. 2018; Ruiz et al. 2016b), but only a fraction of the genetic diversity of quinoa germplasm has been studied. Thus far Ecuadorian quinoa has not been included in salt tolerance studies, and very little is known about its agronomic or breeding value. In this study, we show that Ecuadorian quinoa adds valuable agronomical and salt tolerance traits to quinoa germplasm that can support breeding programs towards an even more resilient and nutritional crop with stable high yields.

\subsection{Diversity of Ecuadorian quinoa for agronomical traits}

The broad genetic diversity of quinoa has been associated with the environmental variation at its centre of origin, the agricultural practices and the exchange of seeds by local communities of the Andean Region (Planella 2015). According to the classification of quinoa in ecotypes, Ecuador is the centre of origin for Inter-Andean valley quinoa; an ecotype that grows in areas between 2300 and 3500 m.a.s.l., characterized by annual rainfall between 700 and $1500 \mathrm{~mm}$ (Bazile et al. 2016b). The Inter-Andean valley ecotype is described as a large and diverse group formed by late-maturing, short-day landraces that are typically branched, tall, have large panicles, large seeds and high yields in terms of both green biomass and grain (Risi and Galwey 1989). The collection of Ecuadorian quinoa genotypes evaluated in this study displayed high variation for seed yield and other agronomical traits. The average worldwide farm yield reported for quinoa is 2 ton/ha (Mastebroek et al. 2002), while the current potential yield on the field can be as high as $4-5$ ton/ha (Zurita-Silva et al. 2014). In the controlled environment used in this study average seed yield under non-stress conditions was $780 \mathrm{~g} / \mathrm{m}^{2}$ (=7.8 ton/ha), with some genotypes even as high as $1200 \mathrm{~g} / \mathrm{m}^{2}$ (12 ton/ha). Although these high yields may be indicative for the high potential of quinoa germplasm from the tropical areas, it should be noted that the plants in this study were grown using optimal crop density, and inside a greenhouse with controlled light conditions, temperature, optimal nutrient availability and irrigation. The performance of these lines should also be evaluated in the field under short-day conditions to validate their yield potential under farm conditions. In addition to yield, seed size (estimated by the TSW), varied strongly. TSW of the tested Ecuadorian quinoa genotypes had a broad range (2.2$4.4 \mathrm{~g} / 1000$ seeds), and for some genotypes it was significantly higher than the large 
seed sizes of the current EU commercial varieties of quinoa (up to $3 \mathrm{~g} / 1000$ seeds) (Jacobsen and Christiansen 2016). Seed size is an important breeding target because of consumer preference for larger seeds and because it increases the biomass of the crop at the same plant density. A higher TSW, therefore, increases productivity. Another important seed quality trait is saponin content. Seeds of quinoa usually contain saponins in the seed coat, resulting in a bitter taste. During the processing of quinoa seeds, saponins need to be removed from the grain, which is laborious and expensive, and requires fresh water. High diversity in quinoa seed saponin content has been reported, so the development of low or free-saponin seed is a main target for the breeding of the crop (Mastebroek et al. 2000). As reported in Table 1, our collection shows broad diversity in the saponin content, and many lines had little or no saponins in their seeds based on the afrosimetric test. Evidence suggest that seed bitterness is controlled by a single dominant gene (TSARL1) (Jarvis et al. 2017), and can therefore easily be selected in a breeding program. Further research is required to test for possible allelic variants of the gene that might explain the diversity for this trait in the Ecuadorian material evaluated in this study.

\subsection{Diversity of Ecuadorian quinoa for salt tolerance}

The mean STI for the Ecuadorian genotypes was $78 \%$, and 13 genotypes out of 22 even showed no significant difference for seed yield between control and 250 $\mathrm{mM} \mathrm{NaCl}$ stress conditions. Only two genotypes had an STI lower than $50 \%$. This high salt tolerance may be linked to the nature of Ecuadorian soils. While the impact of soil salinization for agriculture in the Andean region of Ecuador has not been assessed, it is known that soils in this area have a volcanic origin, and suffer from natural pyroclastic salinity as well as artificial salinity caused by intense irrigation in agricultural areas. It is reported that about $20 \%$ of the soils of the Andean region of Ecuador are indurated soils with high contents of salts (mainly carbonates), commonly affected by erosion, and constituting about $10 \%$ of the total agricultural area (Zebrowski 1996). No association was found between the salt tolerance and the geographical locations where these genotypes were collected, which may be explained by the dissemination of genetic material throughout the Andean provinces in Ecuador via informal seed networks.

Even though the seed yield under control conditions was positively correlated with the seed yield under stress conditions $(r=0.48, p<0.05)$, a significant genotype $x$ salt stress interaction was found for this trait. Some of the high yielding genotypes under control conditions, like 5-Chimborazo and 4-Chimborazo, had very low yields under stress conditions. This means that for breeding purposes, the selection of 
genotypes with high salt tolerance cannot be done by evaluating the material under control conditions only. Nevertheless, several genotypes had stable yields under both conditions as well as favorable agronomical properties such as $\mathrm{HI}$ and TSW. Specifically, the genotypes 1-Azuay, 3-Imbabura, 1-Chimborazo, 1-Imbabura and 1Canar had high yields under control conditions and were able to maintain a similar yield under saline conditions. Whether these genotypes are also the best performers under field conditions and soils affected by salinity remains to be tested.

The genetic diversity for salt tolerance of other quinoa ecotypes has been explored in other studies. Adolf et al. (2012) studied the growth of quinoa genotypes from Bolivia, Peru and breeding material from Denmark under a salt treatment of 400 $\mathrm{mM} \mathrm{NaCl}$ (Adolf et al. 2012). They concluded that higher salt tolerance was found in some but not all accessions related to the Real type, a group of cultivars grown and adapted to the Bolivian salt flats. In a different study, the diversity of quinoa from Peru, Bolivia and Denmark was evaluated under $400 \mathrm{mM} \mathrm{NaCl}$ and it was concluded that the most tolerant varieties were originating also from saline areas from Peru and Bolivia (Shabala et al. 2013). Schmöckel and collaborators reported the salt tolerance of highland and coastal accessions from Chile, Argentina, Peru and Bolivia at $300 \mathrm{mM} \mathrm{NaCl}$ and no clear association between the salt tolerance and geographical location of the genotypes was found (Schmöckel et al. 2017). The performance of different quinoa genotypes under moderate levels of salinity (150$250 \mathrm{mM} \mathrm{NaCl}$ ) has also been explored. A $50 \%$ seed yield reduction was reported for the Danish variety Titicaca under field conditions (Razzaghi et al. 2011). The seed yield reduction of Chilean lowland quinoa ranged from 34 to $74 \%$ under $300 \mathrm{mM}$ $\mathrm{NaCl}$ (Peterson and Murphy 2015a). In summary, compared to other ecotypes previously studied, the Inter-Andean valley ecotype seems to be highly salt tolerant. The national germplasm bank (INIAP) maintains an unexplored collection of Ecuadorian genotypes collected in the 1980s' (Peralta E 2015). A further characterization of this collection will be very interesting as we now have shown large genetic variation in a limited set of Ecuadorian accessions, which gives high promise for the more extensive INIAP collection.

Two Dutch cultivars adapted to grow under long day, temperate conditions (Pasto and Red Carina) were included in our evaluation to be compared with Ecuadorian genotypes. The seed yield of Pasto was considerably lower than that of the Ecuadorian genotypes, but also than yields obtained for this variety in other experiments (Supplementary Table 3). The competition for light may have contributed to the lower yield of Pasto measured here, as Pasto is a small variety with a measured height of $45 \mathrm{~cm}$ while the average height of the Ecuadorian 
genotypes was $172 \mathrm{~cm}$, resulting in shading of the Pasto plots by the Ecuadorian plots. In addition, Pasto is adapted to a long-day photoperiod. Under the artificial short-day photoperiod used in this experiment, Pasto started flowering only 54 days after sowing and completed its crop cycle in 96 days, while under a long-day photoperiod, Pasto completes its cycle in approximately 5 months (Jaramillo Roman et al. 2020) (See also Chapter 5). This shortened crop cycle has likely further contributed to the low yield of Pasto in this study.

\subsection{Salt tolerance mechanisms in Ecuadorian quinoa}

All the Ecuadorian genotypes proved to be salt tolerant, but considerable variation in the level of tolerance was found, as well as Genotype $x$ Treatment interactions.

All the genotypes evaluated in this study relied at least partly on $\mathrm{Na}^{+}$exclusion from leaves as the main strategy to cope with salinity. For the first two months of salt stress, the $\mathrm{Na}^{+}$concentration in young leaves was considerably lower than that in the root medium. It has been reported that the exclusion of $\mathrm{Na}^{+}$has a high energetic cost for plants (Munns and Gilliham 2015), but at the salt stress level applied in this study it seems to be an effective strategy of quinoa to cope with salinity while maintaining growth, at least for a large part of the growth cycle. After three months of stress, some genotypes accumulated very high concentration of $\mathrm{Na}^{+}$in leaves, indicating that these genotypes change strategies to tissue tolerance after prolonged stress. Previously, we reported that tissue tolerance is a favoured strategy of quinoa to survive severe salt stress (Jaramillo Roman et al. 2020). Our results here confirm the suggestion from other chapters that $\mathrm{Na}^{+}$exclusion enables quinoa to grow effectively under mild (for quinoa) salt stress, but that under severe or prolonged stress growth is no longer prioritized, and a strategy aimed at survival (tissue tolerance) at the cost of growth is preferred.

In most of the tested genotypes $\mathrm{Na}^{+}$appeared to be actively excluded from the leaves throughout the season, while the concentration of $\mathrm{Cl}^{-}$in leaves was high from the beginning of the season. Plants typically accumulate higher levels of $\mathrm{Cl}^{-}$than $\mathrm{Na}^{+}$under saline conditions, which may be related to a lower toxicity of $\mathrm{Cl}^{-}$compared to $\mathrm{Na}^{+}$(Flowers and Colmer 2008). $\mathrm{Cl}^{-}$may serve as an osmoticum in the tissues and also balances charge and voltage for the changes in $\mathrm{Na}^{+}$and $\mathrm{K}^{+}$cation concentrations (Teakle and Tyerman 2010).

When exposed to high levels of salinity, quinoa must meet the challenge of osmotic adjustment to a lower external water potential (Flowers and Colmer 2008). Osmotic adjustment by the accumulation of inorganic solutes has been reported as 
the main salt tolerance strategy in halophytes and salt tolerant crops like barley (Munns and Gilliham 2015). Considering the high $\mathrm{Na}^{+}$exclusion activity of the Ecuadorian genotypes, $\mathrm{Na}^{+}$probably only contributed to the osmotic adjustment of quinoa after prolonged salt stress. $\mathrm{K}^{+}$may also play an important role in osmotic adjustment of quinoa. While most halophytes, like species from the genus Suaeda, Atriplex and Inuda, show a decrease in the $\left[\mathrm{K}^{+}\right]$in leaves as a consequence of salt stress, quinoa appears to be unique in its ability to increase $\left[\mathrm{K}^{+}\right]$under highly saline conditions, maintaining a high $\mathrm{K}^{+} / \mathrm{Na}^{+}$ratio. This remarkable ability is likely to protect metabolic processes that require $\mathrm{K}^{+}$from competition by $\mathrm{Na}^{+}$while also adjusting osmotic values in the cytosol to match accumulated of ions in the vacuole. Osmotic adjustment in quinoa may occur mainly through the accumulation of $\mathrm{Cl}^{-}$and $\mathrm{K}^{+}$; however, especially after short exposure to salt stress organic solutes might still be needed to adjust to the lower external water potential caused by the salt treatment.

Salt stress has a strong negative effect on the transpiration of plants (Hedrich and Shabala 2018). While stomatal conductance was reduced in all genotypes, we did observe variation in the degree of reduction of stomatal conductance ( $g s$ ) (Figure 9). It has been suggested that a slight reduction in gs can enhance photosynthetic efficiency and increase WUE, reducing water loss while $\mathrm{CO}_{2}$ uptake is hardly compromised (Yoo et al. 2009). The reduction of $g s$ did was correlated with many agronomical and physiological traits especially at the second measuring point and the genotypes with the strongest gs reduction had the lowest STI (Figure 10), suggesting that the degree of stomatal closure impacts salt tolerance.

Our results demonstrate that the Ecuadorian quinoa genotypes utilise different strategies to grow under saline conditions and to regulate ion dynamics throughout the growing cycle. 4_Azuay and 1_Canar were both highly salt tolerant genotypes, with the same STI of 0.8. 4_Azuay had a relatively high reduction of gs (30\%), low leaf $\mathrm{Na}^{+}$and $\mathrm{Cl}^{-}$concentrations and high leaf $\left[\mathrm{K}^{+}\right]$; similar adaptations as those reported in Pasto (see chapters 2 and 5). On the other hand, 1_Canar had a low reduction of $g s(15 \%)$, high leaf $\mathrm{Na}^{+}$and $\mathrm{Cl}^{-}$concentrations and relatively low leaf $\left[\mathrm{K}^{+}\right]$. Previous reports on genetic diversity of salt tolerance traits in quinoa concluded that the most salt tolerant genotypes had a lower $\mathrm{Na}^{+}$loading to the xylem (Adolf et al. 2012; Shabala et al. 2013). While our data also point to $\mathrm{Na}^{+}$exclusion as an essential salt tolerant mechanism in quinoa, salt tolerance of this species does not only rely on the ability of the genotypes to exclude $\mathrm{Na}^{+}$. The best performing genotypes (in terms of yield) under both control and saline conditions (1-Azu, 3-Imb, 1-Chi, 1-Imb, 1-Can) were mainly characterized by a lower gs reduction, lower $\mathrm{K}^{+}$ 
retention and higher $\mathrm{Na}^{+}$content in leaves. This appeared to be the most effective strategy at this relatively mild (for quinoa) level of stress. The most preferred mechanisms of quinoa to thrive under saline conditions may be dependent on the salinity level and an optimal balance between conservation and growth.

\subsection{Breeding potential of Ecuadorian quinoa}

Despite the interesting properties of the Inter-Andean valley ecotype, Ecuadorian quinoa has hardly been included in scientific research, and its commercial expansion is limited. The strict photoperiod dependence of this ecotype has limited its adaptation to grow at latitudes with long days. The Inter-Andean valley quinoa requires short days (11-13 h light) to induce anthesis. Bolivian quinoa, on the other hand, is more flexible and flowers in regions with a broader range of photoperiods. Cultivars from southern Chile are the least sensitive to photoperiod (Jacobsen 2003). Most of the commercial varieties developed to grow in the northern hemisphere have inherited insensitivity to photoperiod from Chilean quinoa (Bazile 2015), and these can be used to confer this insensitivity to Ecuadorian genotypes as well. While other quinoa ecotypes (Salares, Coastal/lowland) have been broadly recognized for their high salt tolerance attributed to the extreme conditions of their native areas (Ruiz et al. 2016a), this study showed the potential of the Inter-Andean valley ecotype as an alternative source for salt tolerance. Despite the limited number of genotypes, high variation was found for all the examined traits. Several strategies to cope with salinity were identified and, in comparison to European commercial varieties, the genotypes showed high diversity for yield, yield stability and other agronomical traits of interest. Low quality seed, low degree of germination and restricted access to seeds have been identified as limiting factors for the experimentation and worldwide expansion of quinoa in general (Bazile et al. 2016b) and the Inter-Andean valley ecotype in particular. The strengthening of research consortiums, local and international seed reservoirs and participatory breeding programs are needed to facilitate a broader distribution of this crop and a proper characterization of all of its diversity and potential (McElhinny et al. 2007; Murphy et al. 2016). 


\section{Acknowledgments}

The authors gratefully acknowledge the expert assistance of Geurt Versteeg in the greenhouse experiments. The support of Carlos Cobo and Antonio Riofrio during the germplasm collection in Ecuador is also acknowledged. Viviana Jaramillo is financially supported by a scholarship from the Secretariat for Higher Education, Science, Technology and Innovation of the Republic of Ecuador (Contract Number 2015-AR2Q8878). Germplasm access and research permit was granted by the Ministry of Environment of Ecuador (MAE-DNB-CM-2016-0044). The export and cultivation of the Ecuadorian germplasm in the Netherlands was authorized by the export permit No.011-2017-EXP-IC-FLO-DNB/MA with the corresponding MTA. 


\section{Supplementary material}
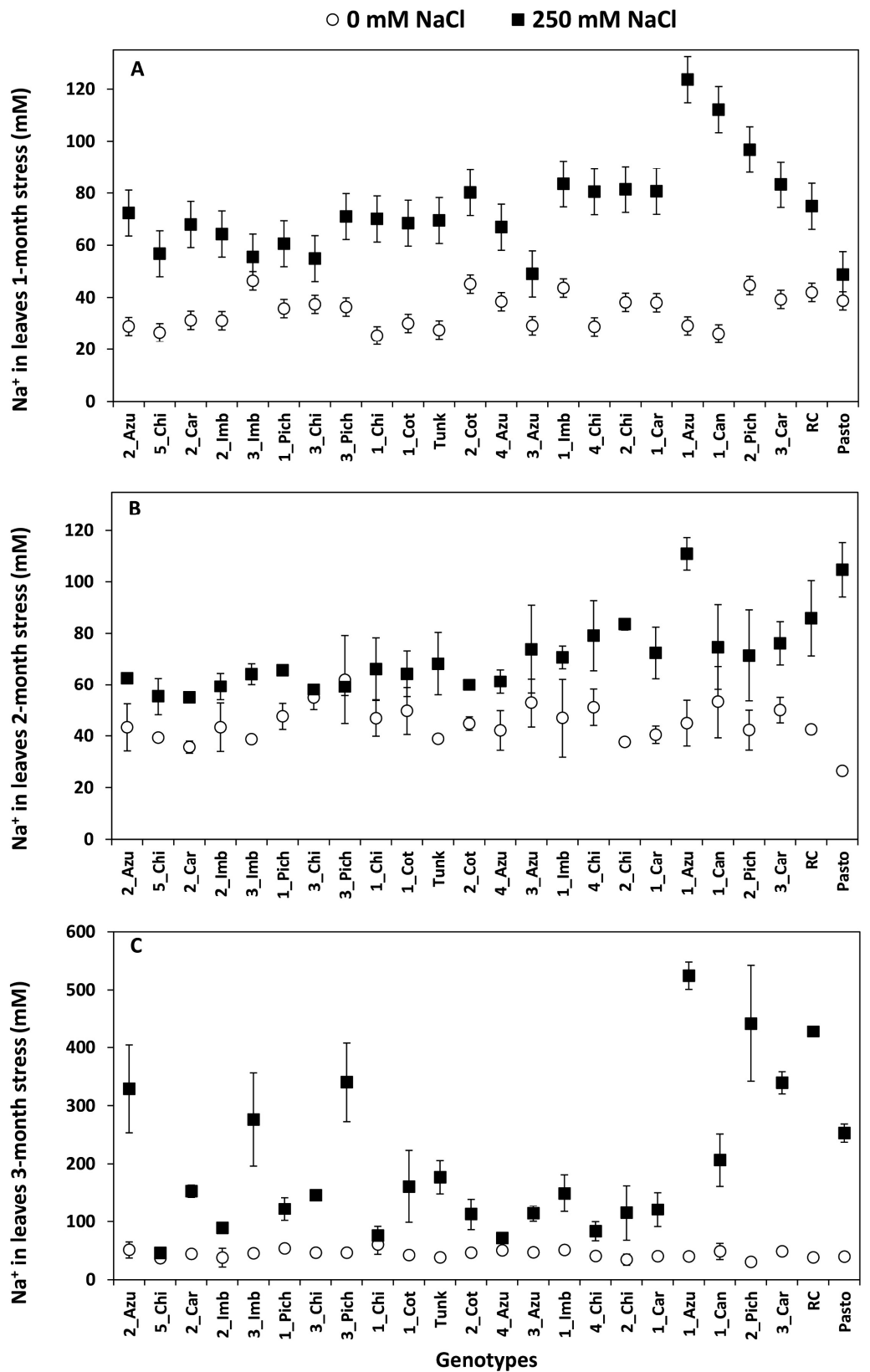

Figure S1. lon contents $\left(\mathrm{Na}^{+}, \mathrm{K}^{+}\right.$and $\left.\mathrm{Cl}^{-}\right)$in young leaves one, two and three months after the start of the salt treatment. Means of two plots per genotype and two measurements per plot. Error bars indicate SE of individual means. 

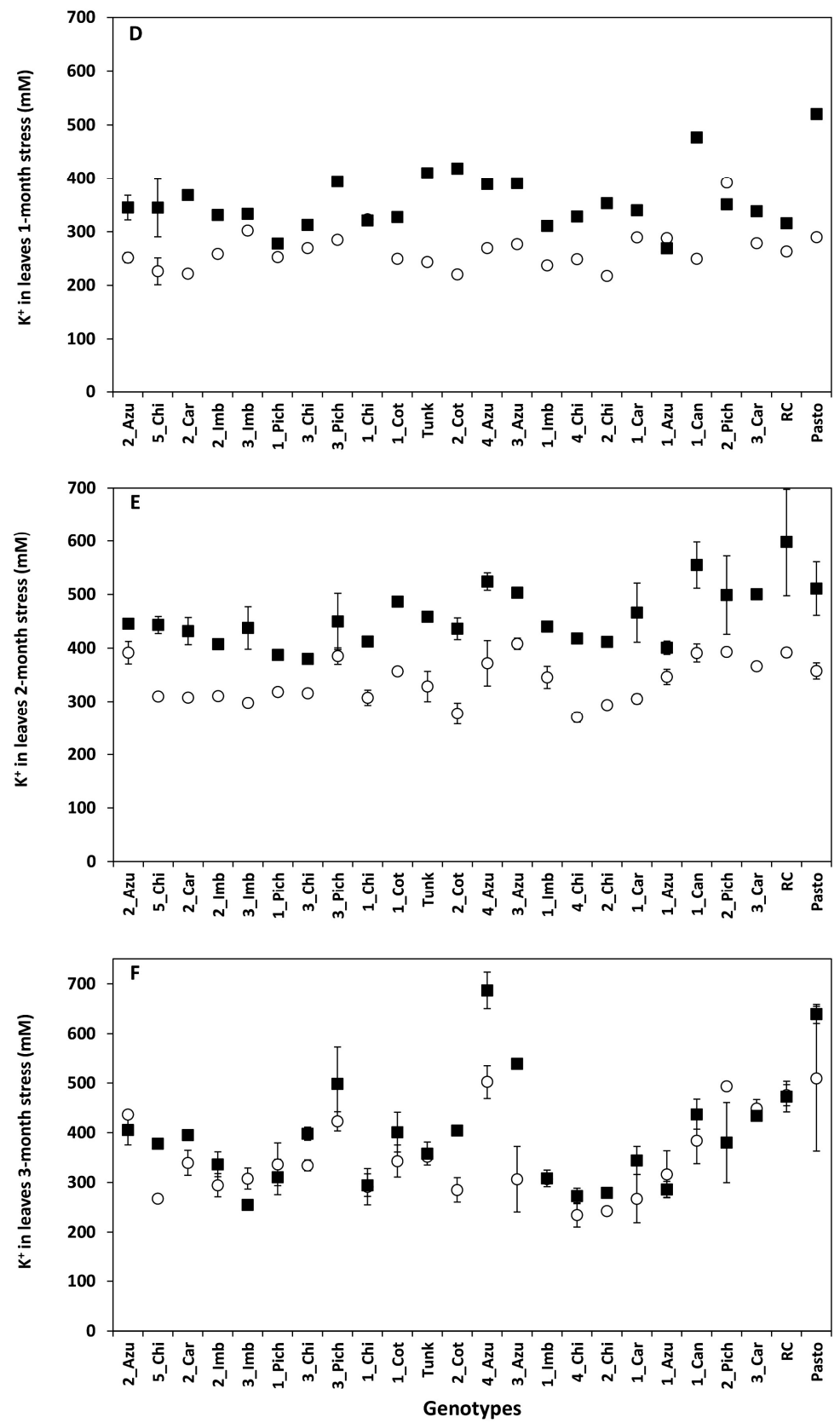

Figure S1 (continued) 

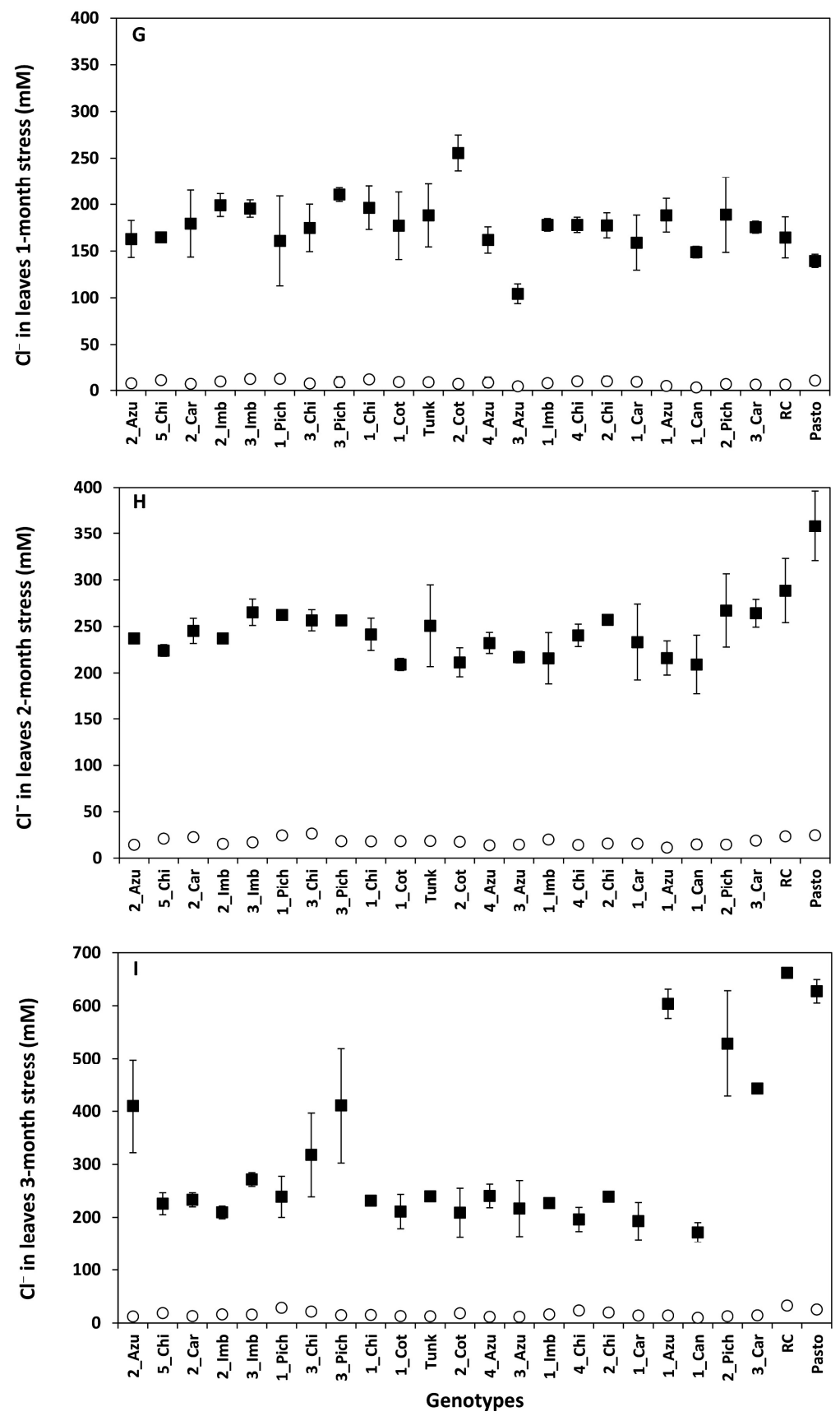

Figure S1 (continued) 


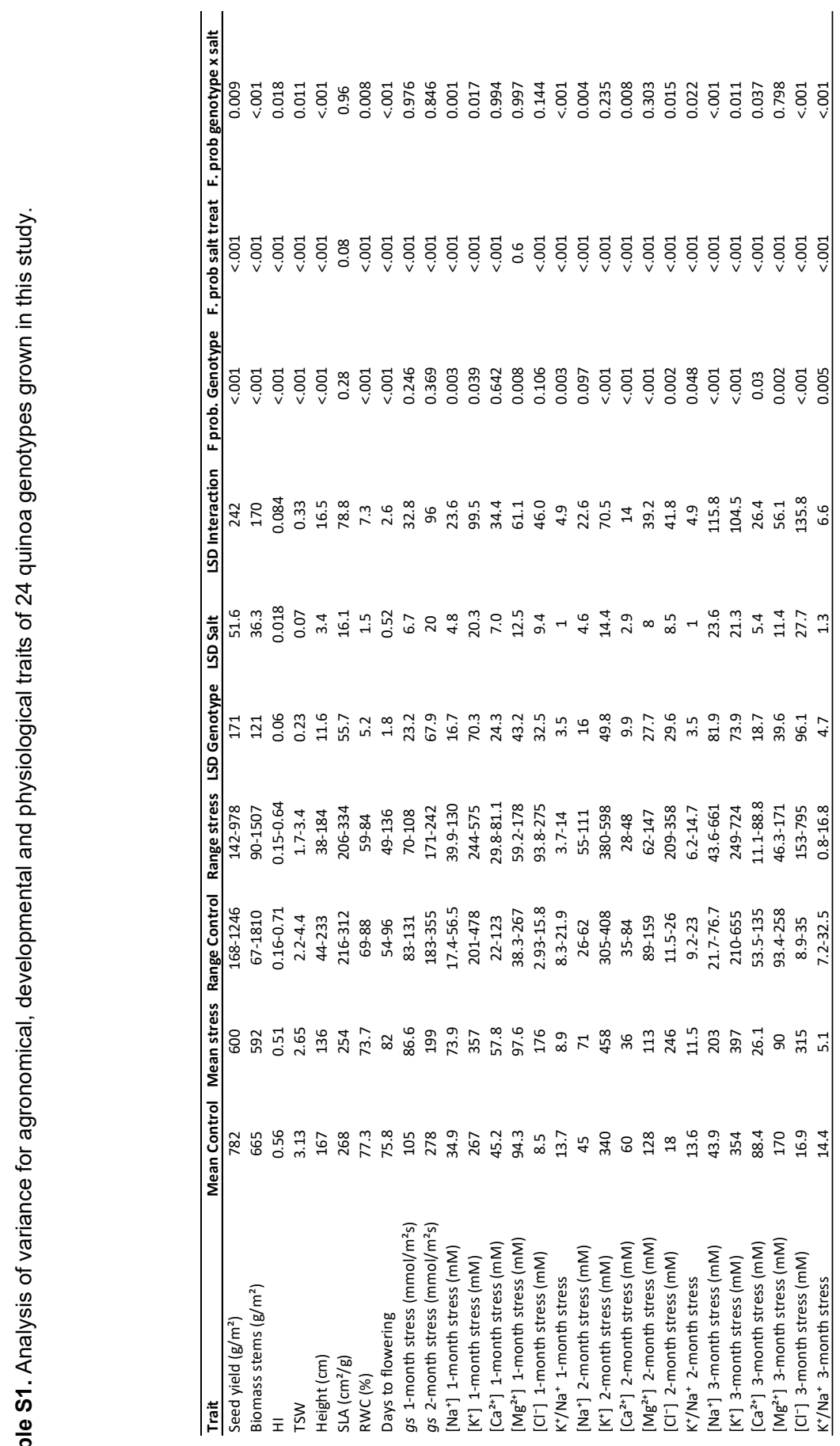


Table S2. Information of the eigenvalues, variance and contribution of variables for the PCA for salt tolerance traits in the collection of Ecuadorian quinoa.

\begin{tabular}{|c|c|c|c|c|}
\hline & Eigenvalue & Variance\% & Cumulative variance $\%$ & Order of contributing variables \\
\hline PC1 & 3.56 & 27.4 & 27.4 & $\begin{array}{l}\mathrm{K}^{+} / \mathrm{Na}^{+} \_2 \text { month_S, } \mathrm{K}^{+} / \mathrm{Na}^{+} \_1 \text { month_S, }\left[\mathrm{K}^{+}\right] \_2 \text { month_S, }\left[\mathrm{K}^{+}\right] \text {1 } \\
\text { month_S, }\left[\mathrm{Na}^{+}\right] \_1 \text { month_S, }\left[\mathrm{Na}^{+}\right] \_1 \text { month_S, }\end{array}$ \\
\hline PC2 & 3 & 23.13 & 50.53 & {$\left[\mathrm{Na}^{+}\right]$_2 month_S, $\left[\mathrm{Na}^{+}\right] \_1$ month_S, RWC_S, STI } \\
\hline PC3 & 1.67 & 12.9 & 63.43 & {$\left[\mathrm{~K}^{+}\right] \_1$ month_S, $\left[\mathrm{Cl}^{-}\right] \_2$ month_S, $\left[\mathrm{Na}^{+}\right] \_1$ month_S, $\left[\mathrm{K}^{+}\right] \_2$ month_S } \\
\hline PC4 & 1.6 & 12.34 & 75.77 & {$\left[\mathrm{Cl}^{-}\right] \_2$ month_S, Decrease gs_1 month, SLA_S } \\
\hline PC5 & 1.01 & 7.8 & 83.57 & \\
\hline PC6 & 0.65 & 5.03 & 88.6 & \\
\hline PC7 & 0.56 & 4.29 & 92.89 & \\
\hline PC8 & 0.38 & 2.95 & 95.84 & \\
\hline PC9 & 0.22 & 1.71 & 97.55 & \\
\hline PC10 & 0.18 & 1.43 & 98.98 & \\
\hline PC11 & 0.11 & 0.86 & 99.84 & \\
\hline PC12 & 0.01 & 0.09 & 99.93 & \\
\hline PC13 & 0.005 & 0.04 & 99.97 & \\
\hline
\end{tabular}

Table S3. Comparison of yield of Dutch varieties Pasto and Red Carina in different field and greenhouse experiments carried out in the last few years.

\section{Seed yield in $\mathrm{g} / \mathrm{m}^{2}$}

\begin{tabular}{l|r|r|r|r} 
& $\mathbf{2 0 1 6}$ & $\mathbf{2 0 1 7}$ & $\mathbf{2 0 1 8}$ & $\mathbf{2 0 1 9}$ \\
\cline { 2 - 5 } Pasto & 363.7 & 306.7 & 324.8 & 168.20 \\
Red Carina & 334.7 & 225.5 & 649.3 & 604.60
\end{tabular}

2016 and 2017: field trials in Wageningen, The Netherlands

2018: experiment in a tunnel, semi-controlled conditions, plant-density 50 plants $/ \mathrm{m}^{2}$

2019: (this experiment) greenhouse experiment, short-day conditions, plant-density 100 plants $/ \mathrm{m}^{2}$ 



\section{Chapter 7}

General discussion 
Every year up to 10 Mha of arable land are abandoned due to soil salinization (Srivastava et al. 2019). This loss of arable land poses a threat to food security in many areas and comes at a great economic loss. Therefore, investments in more salt tolerant crops is not only economically worthwhile but also a necessity to secure food supply. Improvement of salt tolerance of existing crops might not be sufficient under increasing salinization and exploration of new, more salt-tolerant or even halophyte species might be needed. Among potential candidates for such new crops, quinoa (Chenopodium quinoa) is especially interesting. Native to the harsh landscapes of the Andes, quinoa is a highly resilient crop that has been proposed as a model species for salt tolerance. In addition, quinoa is a highly nutritious food crop, thus an interesting target for breeders and farmers to be cultivated in salinized areas around the world. In this thesis I aim to provide novel insights into the salt tolerance of quinoa, genetic differences in salt tolerance mechanisms and how growth and seed yield are balanced under different levels of salinity. In Chapters 2, 3 and 4 we compared physiological, biochemical and growth-related traits between several European commercial varieties exposed to a wide range of salt stress levels (EC in the root medium from 10-65 dS/m). All the evaluated varieties could produce grain even at the highest salt stress applied. However, clear differences in the salt tolerance index (harvestable yield under salinity relative to yield without salinity) and mechanisms of salt tolerance were observed between varieties. The experiments described in Chapters 2, 3, and 4 were performed under greenhouse conditions using single plants as experimental units, allowing a controlled and detailed examination of different complex traits related to salt tolerance. To evaluate the performance of the varieties in an environment more similar to field conditions, the plants were grown with an optimal crop-like plant density $\left(\sim 50\right.$ plants $\left.\mathrm{m}^{-2}\right)$ and the results of this trial are described in Chapter 5. However, to better manage the environmental conditions and control salt concentrations in the root medium, plants were grown in a tunnel using cocopeat as substrate. This approach allowed us to examine the agronomical impact of salt tolerance in addition to the physiological adaptations. In the same setup, two biparental crosses from varieties with contrasting salt tolerance responses were evaluated to perform a preliminary exploration of the genetic determinants for traits contributing to salt tolerance. Finally, in Chapter $\mathbf{6}$ we evaluated Ecuadorian genotypes to test the potential of the Inter-Andean valley ecotype as a new source of traits of interest for the improvement of quinoa. In this thesis I incorporated a wide range of tools and approaches to explore salt tolerance across different experimental spatial scales (from plant to plot) and different levels of salt stress. While a salinity level above $4 \mathrm{dS} / \mathrm{m}(\sim 100 \mathrm{mM}$ 
$\mathrm{NaCl}$ ) in the soil affects the yield of most of the current crops, in my studies described in this thesis a concentration of $200-250 \mathrm{mM} \mathrm{NaCl}$ was considered as moderate stress, and a concentration from $300-400 \mathrm{mM} \mathrm{NaCl}$ as high stress for quinoa.

Quinoa is an excellent crop to study salt tolerance mechanisms. In the following pages I intend to summarize what we have learned and my perspectives on the subject. This chapter is divided in three sections. First, I summarize the main responses of quinoa to salt stress. Next, I discuss the prospects of salt stress research and the advantages of using quinoa as a salt tolerant model. Finally, I analyse the current status of quinoa cultivation and how studying salt tolerance might contribute to the expansion and consolidation of this crop as a staple commodity.

\section{Part 1: Distinctive features that confer salt tolerance to quinoa}

\subsection{Ion dynamics}

The uptake of ions and their distribution within different tissues are essential processes in the response to salinity stress at whole plant level. Through the different experiments within this thesis we evidenced that the uptake, transport and accumulation of ions in quinoa are fine-tuned processes in time (throughout the growing cycle) and space (between plant tissues). In this section, I analyse and compare the distribution of $\mathrm{Na}^{+}, \mathrm{Cl}^{-}$and $\mathrm{K}^{+}$in the different plant tissues between plants under control conditions and plants exposed to moderate and high salt stress (Figure 1). While in Chapter 2 ion dynamics throughout the growing season of plants were compared, here I will examine the ion dynamics at one particular time point (10-12 weeks after sowing, 5-7 weeks after stress application, beginning of flowering in plants), and compare responses at different levels of salt stress (data from Chapters 2, 4 and 5). 


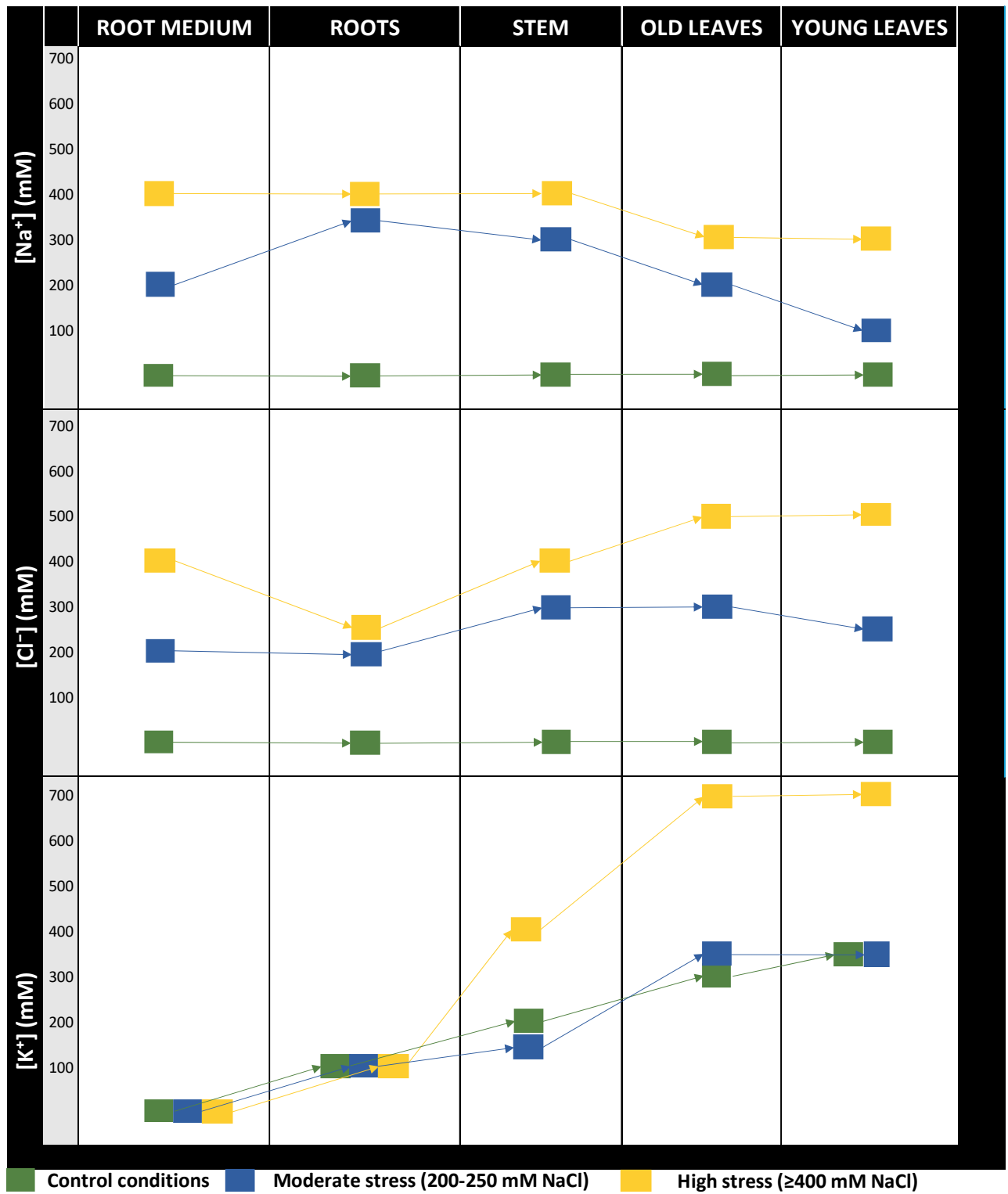

Figure 1. Schematic representation on $\mathrm{Na}^{+}, \mathrm{Cl}^{-}$and $\mathrm{K}^{+}$distribution in quinoa plants tissues under moderate (from Chapters 5, 6) and high (from Chapters 2, 4) salt stress at the onset of flowering (10-12 weeks after sowing, 5-7 weeks after stress application). 
In the upper panel of Figure 1, the $\mathrm{Na}^{+}$distribution in different tissues under moderate and high salt stress is shown. The concentration of $\mathrm{Na}^{+}$in the roots equalled that of the root medium in plants growing under high stress, and was slightly higher than the root medium in plants growing under moderate stress. At both salinity levels the $\left[\mathrm{Na}^{+}\right]$in roots was higher than in stems, and in stems higher than in leaves. Under moderate salt stress, old leaves accumulated more $\mathrm{Na}^{+}$than young leaves, while under high stress less differences were found between old and young leaves. The concentration of $\mathrm{Na}^{+}$in leaves (especially young leaves) was considerably lower than in the root medium even under severe salinity, which points to an extraordinary capacity of quinoa to exclude $\mathrm{Na}^{+}$from leaves (See section 1.2). While most of the genotypes kept leaf $\left[\mathrm{Na}^{+}\right]$lower than in the root medium, slightly higher levels of $\mathrm{Na}^{+}$ in the leaves correlated with higher stomatal conductance and higher yields under moderate salt stress: these genotypes were able to maintain their growth rate in spite of the salt stress. Other genotypes retained low levels of $\mathrm{Na}^{+}$in young leaves; among the varieties Pasto stands out for this trait that was also observed in some of the Ecuadorian genotypes evaluated in Chapter 6, such as 4_Azuay. The interaction between these different patterns of $\mathrm{Na}^{+}$accumulation and growth is discussed later (See section 1.6). The two mapping populations studied in Chapter 5 (Pasto $x$ Red Carina (PxRC) and Atlas $x$ Red Carina (AxRC)) segregated for $\left[\mathrm{Na}^{+}\right]$in the shoot and the lowest levels of $\mathrm{Na}^{+}$were measured in some genotypes of the progeny of PxRC. QTLs were found for this $\mathrm{Na}^{+}$exclusion trait and alleles inherited from Pasto were identified, which point to possible genetic determinants of the higher exclusion rate of Pasto that deserve further exploration.

The ability to exclude $\mathrm{Na}^{+}$from tissues is one of the most important features that allow halophytes to grow in saline environments (Flowers and Colmer 2008). Two important questions are pivotal to understand the mechanism of $\mathrm{Na}^{+}$exclusion: where in the plant does the exclusion occur, and how is it facilitated? $\mathrm{Na}^{+}$exclusion from leaves involves the ability of the plant to: 1) minimize $\mathrm{Na}^{+}$entry; 2) maximize efflux to the root media; 3 ) minimize loading to the xylem; 4) maximize retrieval from the xylem; and 5) maximize recirculation out of the shoot into the phloem (Tester and Davenport 2003). The $\mathrm{Na}^{+}$concentrations in the roots of quinoa were similar to the root media at high salinity, and remained constant throughout the growing cycle. When soil salinity is high, $\mathrm{Na}^{+}$influx into the roots is passive, favoured by differences in concentration and voltage (Munns et al. 2020a). It is possible that $\mathrm{Na}^{+}$ accumulated in the quinoa roots through passive transport until reaching an equilibrium with the root medium, which will also facilitate the uptake of water. The entry of $\mathrm{Na}^{+}$into the root cells is mediated by uniporters such as LCT and NSCC 
(Munns et al. 2020a). The root $\mathrm{Na}^{+}$concentration will not rise anymore when efflux of the additional $\mathrm{Na}^{+}$to the root medium balances the influx (Britto and Kronzucker 2015). The efflux of $\mathrm{Na}^{+}$to the root media is an energetically expensive process, probably mediated by a $\mathrm{Na}^{+} / \mathrm{H}^{+}$antiporter; until now, the only transporter known to be associated with $\mathrm{Na}^{+}$efflux is SOS1 (Britto and Kronzucker 2015). The lower levels of $\mathrm{Na}^{+}$in quinoa leaves compared to roots may at least partly be attributed to prevention of $\mathrm{Na}^{+}$entry into the xylem from the root cortex. The mechanisms of $\mathrm{Na}^{+}$ retrieval from the xylem are largely unknown; one of the most likely candidates involved in this process is the HKT-type 1 transporter that at elevated levels of $\mathrm{Na}^{+}$ works as a $\mathrm{Na}^{+}$uniporter at the root-xylem symplast interface (Peleg et al. 2011). A transcriptome analysis using RNA-seq was performed to identify candidate transmembrane proteins in quinoa roots and leaves, but based on expression profiles, no clear candidates for ion transporters were identified (Schmöckel et al. 2017) Therefore, the molecular mechanisms related to $\mathrm{Na}^{+}$uptake, efflux to the root medium and retrieval from the xylem in quinoa remain unknown.

The middle panel of Figure 1 illustrates the $\mathrm{Cl}^{-}$distribution in different tissues of quinoa at no, moderate and high salinity at the onset of flowering. The $\left[\mathrm{Cl}^{-}\right]$in the roots was similar to that of the root medium under moderate stress, but lower than the root medium under severe stress, which points to different strategies employed by quinoa at different levels of salinity. Under high salinity, $\mathrm{Cl}^{-}$was significantly lower than $\mathrm{Na}^{+}$in the roots, thus electrochemical balance might be achieved by the accumulation of organic acids. Under moderate salinity, the rate of $\mathrm{Cl}^{-}$exclusion was lower, which might also facilitate water uptake and a less important role for organic solutes to contribute to electrochemical balance. The distribution of $\mathrm{Cl}^{-}$within the plant tissues was opposite to that of $\mathrm{Na}^{+}: \mathrm{Cl}^{-}$concentration increased from roots, to stems to leaves, which indicates a lower rate of $\mathrm{Cl}^{-}$exclusion from leaves than $\mathrm{Na}^{+}$ exclusion (though still very high). The higher levels of $\mathrm{Cl}^{-}$than $\mathrm{Na}^{+}$measured in leaves plus the gradient in $\mathrm{Cl}^{-}$concentration from roots to leaves suggest a lower $\mathrm{Cl}^{-}$ toxicity than $\mathrm{Na}^{+}$in quinoa leaves. Under moderate stress, leaf $\left[\mathrm{Cl}^{-}\right]$had a positive correlation with transpiration and yield, implying that its accumulation in leaves without reaching toxic levels (most likely compartmentalized in vacuoles) contributes to higher growth rates (Chapter $\mathbf{5}$ ). It has been reported that high concentrations of $\mathrm{Cl}^{-}$in the shoot can affect plants in several ways. Principally, $\mathrm{Cl}^{-}$had a negative effect on photosynthesis by inhibiting gas exchange and causing a decline in chlorophyll content (Henderson et al. 2014). This kind of damage was not observed in quinoa, demonstrating its capacity to tolerate high $\mathrm{Cl}^{-}$levels in leaves. $\mathrm{Cl}^{-}$also 
affects plant growth by competing for the same ion transporters with $\mathrm{NO}_{3}{ }^{-}$, which might cause $\mathrm{NO}_{3}{ }^{-}$deficiency in the long-term (Teakle and Tyerman 2010). We explored the nitrate concentrations in quinoa leaves and found relevant differences between varieties: the nitrate concentration was not affected by salinity in Pasto, while it was strongly reduced (by $73 \%$ ) in selRiobamba (Chapter 2). Whether the ability of a genotype to maintain high levels of nitrate under salt stress contributes to salt tolerance in quinoa remains to be determined. Future research on $\mathrm{Cl}^{-} / \mathrm{NO}_{3}{ }^{-}$ transporters in quinoa might give insight in the differences in $\mathrm{Cl}^{-}$exclusion rate between salt treatments and its relation with $\mathrm{NO}_{3}{ }^{-}$retention between genotypes. $\mathrm{Cl}^{-}$ exclusion from roots might be controlled by regulating the activity of proton cotransporters (CCC), which are the most important transporters for $\mathrm{Cl}^{-}$uptake ( $\mathrm{Li}$ et al. 2017). Differences in the selectivity for $\mathrm{Cl}^{-}$and $\mathrm{NO}_{3}{ }^{-}$have been suggested for SLAC (Slow-type Anion Channel) and NPF (Nitrate transporter proteins) (Henderson et al. 2014; Li et al. 2017; Munns et al. 2020a).

A major constraint of salt stress for plants is the reduced availability of $\mathrm{K}^{+}$in saline soils due to the abundance of $\mathrm{Na}^{+}$, and the competition of both ions for the same transporters and enzyme binding sites (Shabala and Cuin 2008). The lower panel of Figure 1 shows the distribution of $\mathrm{K}^{+}$in quinoa tissues under control conditions, moderate and high salt stress at the onset of flowering. In all the conditions, an increasing gradient of $\mathrm{K}^{+}$concentration was seen from roots to stems to leaves. It is known that quinoa has the unique feature to maintain a high $\mathrm{K}^{+}$in the shoots under saline conditions, even when $\mathrm{Na}^{+}$concentrations are high (Schmöckel et al. 2017; Shabala et al. 2013). In our experiments, leaf $\left[\mathrm{K}^{+}\right]$was not significantly affected or slightly increased by moderate salinity, and significantly increased by high salinity. In Chapter 2 we reported that under salt levels higher than seawater (55-65 dS/m) the leaf $\left[\mathrm{K}^{+}\right]$increased almost 3-fold. Orsini et al. (2011) reported the highest increase of $\mathrm{K}^{+}$in leaves under a salt treatment of $750 \mathrm{mM} \mathrm{NaCl}$. The ability to maintain $\left[\mathrm{K}^{+}\right]$in leaves seems to be a response of quinoa to moderate stress, while the active accumulation of $\mathrm{K}^{+}$is a strategy to survive extreme saline conditions. $\mathrm{K}^{+}$might contribute to the osmotic adjustment in leaves, and at the same time compensate for the increased levels of $\mathrm{Na}^{+}$under high stress. However, $\mathrm{K}^{+}$retention in leaves under salinity comes at a high metabolic cost. $\mathrm{Na}^{+}$causes a depolarization of plasma membranes, which reduces $\mathrm{K}^{+}$electrochemical gradient (Rubio et al. 2020). In these conditions, it has been estimated that 1-2 mol ATP are required to accumulate $1 \mathrm{~mol}$ of $\mathrm{K}^{+}$under saline conditions (Munns et al. 2020a). We also reported genotypic differences for $\mathrm{K}^{+}$accumulation; the variety Pasto had the highest 
levels of $\mathrm{K}^{+}$at all salt concentrations. The relation between varietal differences in $\mathrm{K}^{+}$ retention and growth is discussed in section 1.6.

\subsection{Estimating the rate of $\mathrm{Na}^{+}$exclusion from leaves}

We have established in this thesis that an important response of quinoa to salt stress is its extraordinary capacity to exclude $\mathrm{Na}^{+}$, especially at (for quinoa) mild salinity. Plants need to exclude almost all the $\mathrm{Na}^{+}$present in the root medium to prevent rapid accumulation of $\mathrm{Na}^{+}$in transpiring leaves to toxic levels. Munns et al. (2020a) argues that even by excluding $98 \%$ of $\mathrm{Na}^{+}$(from entering to the roots or loading into the xylem), the $\mathrm{Na}^{+}$concentration in the shoot will equal that of the root medium within a few weeks. The rate of $\mathrm{Na}^{+}$exclusion from shoots can be estimated using a model for the $\mathrm{Na}^{+}$concentration and the transpiration rate (Munns et al. 2020b). Box 1 describes the strategy and considerations that are taken into account for the estimation of $\mathrm{Na}^{+}$exclusion in quinoa. 


\section{Box 1. Calculation of $\mathrm{Na}^{+}$exclusion from leaves}

The rate of $\mathrm{Na}^{+}$exclusion from leaves was estimated based on measurements of $\left[\mathrm{Na}^{+}\right]$in leaves throughout the season and the transpiration rate of the plants following the ideas from (Munns et al., 2020b). These considerations were taken into account:

- Data from transpiration and plant growth in leaves were obtained from Chapter 4

- Salt stress started five weeks after sowing

- After six weeks of salt stress (11 weeks after sowing) no further daily increase in transpiration was assumed (plant growth reaches a plateau)

- Stress modelled for this example: $200 \mathrm{mM} \mathrm{NaCl}$

- Two varieties with different $\mathrm{Na}^{+}$accumulation patterns were analyzed: Pasto and selRiobamba

- $\left[\mathrm{Na}^{+}\right]$in young leaves were obtained from measurements in Chapter 4 ( 2 and 6 weeks after stress) and Chapters 2 and $\mathbf{5}$ (8, 10 weeks after stress)

Following these considerations, a model for the accumulation of $\mathrm{Na}^{+}$in young leaves based on the exclusion of $\mathrm{Na}^{+}$from the transpiration stream is presented below. Dots indicate the measurements of $\left[\mathrm{Na}^{+}\right]$in young leaves: in grey from Chapter 4 and in black from Chapter 2 and 5 . A blue straight line depicts the modelled course for $\mathrm{Na}^{+}$accumulation in Pasto and a blue dotted line the modelled course for $\mathrm{Na}^{+}$accumulation in selRiobamba based on the estimated rates of exclusion. Following this model, young leaves would equal the $\left[\mathrm{Na}^{+}\right]$in the root media after three weeks of salt stress for selRiobamba and six weeks of salt stress for Pasto.

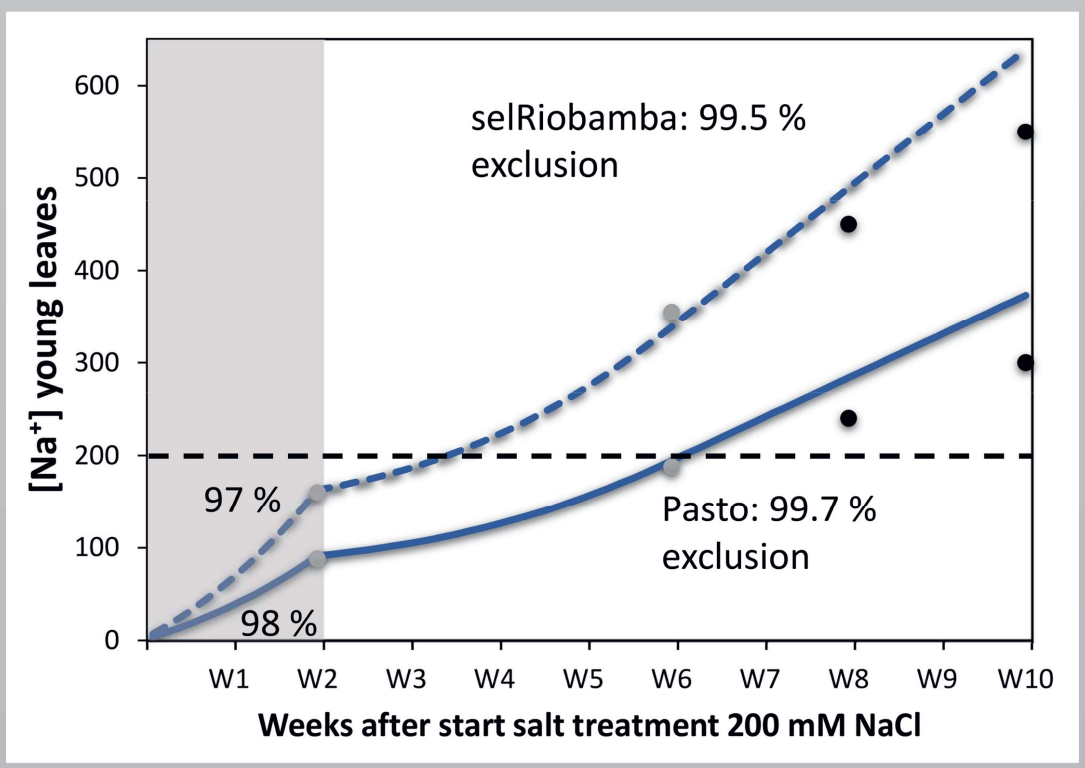


Our data indicate that the uptake rate of $\mathrm{Na}^{+}$in quinoa is slightly higher at the beginning of salt exposure (two weeks of stress) than after the third week of stress (Box 1). This pattern was observed in all the measurements described in Chapters 4 and 6. A higher uptake rate of $\mathrm{Na}^{+}$as a relatively early response to salt stress has been reported for other species, like barley (Munns et al. 2020b). This higher uptake of ions as an early response to salt stress may support osmotic adjustment, thus countering the effects of osmotic stress. After this initial phase, the plants might increase the $\mathrm{Na}^{+}$exclusion rate to prevent toxic concentrations in the leaves. Based on our data, keeping the level of $\mathrm{Na}^{+}$as low as possible appeared to be especially important during flowering; this was observed in all the experiments and genotypes examined.

The predicted $\mathrm{Na}^{+}$exclusion rates (depicted as lines in Box 1) fits well with the $\mathrm{Na}^{+}$contents measured in leaves (depicted as dots). By using the measured variations in daily transpiration for both varieties (Chapter 4), we determined that the differences in $\left[\mathrm{Na}^{+}\right]$in the leaves from Pasto and selRiobamba are caused by differences in the $\mathrm{Na}^{+}$exclusion rate, more than differences in transpiration rate. According to our predictions, during the first two weeks Pasto excluded $98 \%$ of the $\mathrm{Na}^{+}$in the root medium and selRiobamba $97 \%$. After two weeks, the exclusion rate increased to $99.7 \%$ for Pasto and $99.5 \%$ for selRiobamba. Even at the high exclusion rates estimated in this model, the observed exclusion rate appeared to be even higher (lower $\mathrm{Na}^{+}$content) by the end of the growing season. It is important to notice that the model was made from measurements after two and six weeks of salt stress (Chapter 4), while $\left[\mathrm{Na}^{+}\right]$after eight and ten weeks of stress were obtained from a different experiment (Chapters 2 and $\mathbf{5}$ ). The deviation of the predicted from the measured data could be due to differences between experimental conditions. Other factors, such as a lower transpiration rate at the end of the season, or the accumulation of ions in other tissues (e.g. older leaves) could have also contributed to the differences between predicted and measured $\left[\mathrm{Na}^{+}\right]$.

Osmotic adjustment (OA) is one of the main responses of plants to salt stress or drought (Turner 2018). OA can be achieved by synthesizing organic compounds or by the uptake of solutes from the surrounding medium. Halophytes are characterized by their capacity to use ions for this purpose (Flowers and Colmer 2008). Despite the high exclusion rates of quinoa, $\mathrm{Na}^{+}$and $\mathrm{Cl}^{-}$accumulate in the tissues and this can contribute considerably to $\mathrm{OA}$. In addition, $\mathrm{K}^{+}$also increases, so it can also contribute to osmotic adjustment, especially under high salinity. However, it has been reported that organic osmolytes (especially proline and betaine) also 
contribute to the osmotic adjustment of quinoa (Orsini et al. 2011; Shabala et al. 2012). The contribution of organic osmolytes might be important especially during the first weeks of exposure to salt stress when the concentration of $\mathrm{Na}^{+}$and $\mathrm{Cl}^{-}$in the roots is lower than the root medium (Chapters 2 and 4) and, depending on the exclusion rate, is even lower in the leaves. OA achieved by synthesizing de novo organic solutes has been reported as a energetically expensive process (Munns et al. 2020b). A possibility to avoid or lower this cost would be to make use of solutes already available in the plant. In addition to the ions taken up from the soil, it is suggested that quinoa may use solutes that are stored in epidermal bladder cells (EBCs) (Kiani-Pouya et al. 2017) (See section 1.5). Until now, the content of EBCs has not been measured directly, only by comparing leaves before and after brushing the bladders (Kiani-Pouya et al. 2017; Orsini et al. 2011). Based on this comparison and knowledge from other species, it is known that EBCs contain a broad range of organic solutes, including sugars, alcohols and amino acids (Barkla and VeraEstrella 2015). The ability of quinoa to use solutes stored in bladders for early osmotic adjustment under salt stress would be another example of the adaptive capacity of quinoa to face the environmental challenges of salinity or drought.

\subsection{Exploring the unique machinery of $\mathrm{K}^{+}$selectivity and accumulation in quinoa}

The remarkable capacity to maintain $\mathrm{K}^{+}$under salt stress is one of the most distinctive responses to salinity of quinoa. Revealing the mechanisms and transporters involved in the uptake and distribution of $\mathrm{K}^{+}$in different tissues might enhance our understanding of the role of $\mathrm{K}^{+}$in salt tolerance, and give insight in the (metabolic) trade-offs of increasing $\mathrm{K}^{+}$concentrations in leaves under saline conditions.

We performed an in-silico exploration of $\mathrm{K}^{+}$channels and transporters in the genome of quinoa (Jarvis et al. 2017) and found a remarkably high abundance and diversity of $\mathrm{K}^{+}$transporters compared to other species reported in literature (Lebaudy et al. 2007; Véry et al. 2014). Potassium-ion transport across plant membranes is mediated by several families of transporters and channels. In brief, potassium channels include the Shaker-type channels (9 genes in Arabidopsis) and the Tandem-Pore channels (TPK) (6 genes in Arabidopsis). Potassium transporters include the KUP/HAK/KT transporters (13 genes in Arabidopsis) and the HKT transporters (1 gene in Arabidopsis) (Lebaudy et al. 2007; Shabala and Cuin 2008). The Shaker-type potassium channels are low-affinity $\mathrm{K}^{+}$-selective voltage-gated transporters located at the plasma membranes while TPK channels mediate the 
transport of $\mathrm{K}^{+}$into and out from vacuoles (Lebaudy et al. 2007). KUP/HAK/KT transporters are ubiquitously present in tonoplast and plasma membranes of all plant tissues. They are $\mathrm{K}^{+} / \mathrm{H}^{+}$active symporters involved in high-affinity $\mathrm{K}^{+}$transport and have a pivotal role especially in conditions of $\mathrm{K}^{+}$starvation (Szczerba et al. 2009; Véry et al. 2014). Finally, HKT transporters might have a dual function as $\mathrm{K}^{+} / \mathrm{Na}^{+}$ symporters at low $\left[\mathrm{Na}^{+}\right]$and as $\mathrm{Na}^{+}$specific transporters at higher $\left[\mathrm{Na}^{+}\right]$(Véry et al. 2014).

Table 1 summarizes the $\mathrm{K}^{+}$transporters annotated in the quinoa genome and the potential function inferred from homologues of these transporters in other species (Arabidopsis, barley, rice, pepper, tomato, Mesembryathemum crystallinum, among others). The abundance of $\mathrm{K}^{+}$transporters in the quinoa genome is intriguing. As an example, 9 genes have been annotated as Shaker-type channels in Arabidopsis (Lebaudy et al. 2007), and 11 genes in poplar (Zhang et al. 2010) while in quinoa 17 genes have been annotated with multiple copies for some of these transporters: AKT1 (5 copies), KAT1 (4 copies). This suggests a redundancy of transporters for $\mathrm{K}^{+}$homeostasis. We performed a preliminary evaluation of the expression of these transporters in leaves of quinoa plants growing under high salt stress $(400 \mathrm{mM} \mathrm{NaCl})$, also summarized in Table 1 (unpublished results). Interestingly, the majority of the identified transporters were constitutively expressed in leaves under control and stress conditions. One of the differentially expressed $\mathrm{K}^{+}$ transporters in quinoa leaves was AKT2. This transporter is thought to be involved in $\mathrm{K}^{+}$loading/ unloading into/from phloem sap and is associated with long-distance transport of photo-assimilates (Gajdanowicz et al. 2011). In our exploration, AKT2 was downregulated in young leaves under high salinity. Whether this contributes to avoid $\mathrm{K}^{+}$leaking through the phloem, or influences the source-to-sink balance under salinity remains to be explored. In addition, studying the expression of these transporters in different tissues and different time points throughout the season might clarify their role in salt tolerance of quinoa. In Chapter $\mathbf{5}$, we found QTLs for $\left[\mathrm{K}^{+}\right]$in young leaves. In the PxRC population, some of the identified alleles were inherited from Pasto and possibly relate to the higher leaf $\mathrm{K}^{+}$content of this variety. We explored the position of $\mathrm{K}^{+}$transporters in the genome of quinoa and some of them where located in the vicinity of QTL regions (less than $10 \mathrm{cM}$ apart). As an example, genetic region 3 (upper part of linkage group Combi_A_LG02) contained several QTLs for $\left[\mathrm{K}^{+}\right]$and $\mathrm{K}^{+} / \mathrm{Na}^{+}$under salinity (Chapter 5 ), and also harboured several of the $\mathrm{K}^{+}$transporters listed in Table 1. A closer look to this genomic region might give insight in the contribution of these transporters and possible regulators involved in the $\mathrm{K}^{+}$homeostasis of quinoa under salinity. 
Table 1. Summary of $\mathrm{K}^{+}$transporters annotated in quinoa genome, their reported roles in other species and expression in quinoa leaves under control and salt stress $(400 \mathrm{mM} \mathrm{NaCl})$. Information from other species was summarized from (Lebaudy et al. 2007; Szczerba et al. 2009; Véry et al. 2014). Gene copies annotated in the quinoa genome for each transporter are identified between parenthesis with the prefix AUR).

\begin{tabular}{|c|c|c|c|}
\hline $\mathrm{K}^{+}$transporters annotated in quinoa & Homologues in other species & Role reported in other species & Expression in quinoa leaves \\
\hline \multicolumn{4}{|l|}{ Shaker- type channels } \\
\hline $\begin{array}{l}\text { AKT1 (AUR62033444; AUR62041838; } \\
\text { AUR62030683; AUR62011066; AUR62032344) }\end{array}$ & AKT1 & $\mathrm{K}^{+}$uptake in roots & Constitutive \\
\hline $\begin{array}{l}\text { KAT1 (AUR62030205; AUR62039778; } \\
\text { AUR62021243; AUR62016037) }\end{array}$ & KAT1 & Stomata aperture & Downregulated by salinity \\
\hline AKT2 (AUR62042717; AUR62014210) & AKT2 & Phloem loading-unloading & Downregulated by salinity \\
\hline $\begin{array}{l}\text { SKOR (AUR62042218; AUR62038878; } \\
\text { AUR62018137; AUR62037748; AUR62034475) }\end{array}$ & SKOR & $\mathrm{K}^{+}$translocation to the shoot & Constitutive \\
\hline KAB1 (AUR62020272; AUR62037730) & KAB1 & Not defined & Constitutive \\
\hline \multicolumn{4}{|l|}{ TPK channels } \\
\hline $\begin{array}{l}\text { TPK1 (AUR62021824; AUR62036413, } \\
\text { AUR62036440; AUR62012835; AUR62017082) }\end{array}$ & TPK1 & Vacuolar K $\mathrm{K}^{+}$channel & Constitutive \\
\hline \multicolumn{4}{|l|}{ HAK/KUP/KT transporters } \\
\hline CqPOT5 (AUR62033186; AUR62028032) & ATHAK5 & $\begin{array}{l}\mathrm{K}^{+} \text {uptake in roots from low external } \\
\text { concentrations }\end{array}$ & Constitutive \\
\hline CqHAK19 (AUR62010943; AUR62010943) & OSHAK19 & $\begin{array}{l}\mathrm{K}^{+} \text {uptake in roots from low external } \\
\text { concentrations }\end{array}$ & Constitutive; not expressed \\
\hline CqHAK13 (AUR62040746; AUR62026895) & OSHAK13 & Not defined & Constitutive \\
\hline CqPOT4 (AUR62042858; AUR62017474) & ATKUP3=ATKT4 & $\begin{array}{l}\text { Developmental processes: Turgor- } \\
\text { driven cell expansion }\end{array}$ & Downregulated by salt stress \\
\hline CqHAK7 (AUR62010772; AUR62019774) & OSHAK7 & $\begin{array}{l}\text { Developmental processes: Turgor- } \\
\text { driven cell expansion }\end{array}$ & Not expressed \\
\hline CqPOT2 (AUR62031491; AUR62012363) & ATKUP/HAK/KT2 & $\begin{array}{l}\text { Developmental processes: Turgor- } \\
\text { driven cell expansion }\end{array}$ & Constitutive \\
\hline CqPOT6 (AUR62005353; AUR62014006) & ATKUP/HAK/KT6 & Developmental processes & Upregulated by salt stress \\
\hline CqPOT8 (AUR62042411; AUR62035954) & ATKUP/HAK/KT8 & Developmental processes & Constitutive \\
\hline CqPOT1 (AUR62037170; AUR62025122) & ATKUP/HAK/KT1 & Developmental processes & Not expressed \\
\hline CqHAK12 (AUR62026619; AUR62034910) & OSHAK12 & Developmental processes & Constitutive \\
\hline CqPOT7 (AUR62020155; AUR62001622) & ATKUP/HAK/KT7 & $\begin{array}{l}\mathrm{K}^{+} \text {uptake (available in high } \\
\text { concentrations)/ long distance } \\
\text { transport }\end{array}$ & Constitutive \\
\hline CqPOT12 (AUR62005354; AUR62014007) & ATKUP/HAK/KT12 & Not defined & Not expressed \\
\hline CqHAK17 (AUR62003489; AUR62017798) & OSHAK17 & Not defined & Constitutive \\
\hline \multicolumn{4}{|l|}{ HKT transporters } \\
\hline HKT1 (AUR62027136; AUR62012937) & HKT1 & $\mathrm{Na}^{+}$selective & Not expressed \\
\hline
\end{tabular}




\subsection{The role of Epidermal Bladder Cells (EBCs) revised}

A distinctive anatomical feature of quinoa is the presence of Epidermal Bladder Cells (EBCs) especially in young tissues (seedlings, expanding leaves, buds, meristems, young stems, etc.) (Figure 2). It has been proposed that EBCs function as salt bladders and are able to sequester 1000 -fold more $\mathrm{Na}^{+}$than cellular vacuoles, and can accumulate up to $40 \%$ of the total $\mathrm{Na}^{+}$and $\mathrm{Cl}^{-}$content in leaves (Orsini et al. 2011; Shabala et al. 2014; Kiani-Pouya et al. 2017). Some halophytes (recreto-halophytes) have the capacity to store and secrete salt through salt bladders or salt glands (Dassanayake and Larkin 2017; Yuan et al. 2016). Salt bladders should not be confused with salt glands. Salt bladders are modified trichomes composed of one bladder cell that can be surrounded by stalk cells, while salt glands are more complex multi-cellular structures composed by collecting and secretory cells. Salt secretion is also different between bladders and glands: in bladders salt is sequestered in the vacuoles of these specialized cells; eventually, the bladders break and the salt is deposited in the surface of the leaves where it can be washed out by rain or wind. (Dassanayake and Larkin 2017). The proportion of salt excreted through bladders depends on the species. For example, the salt marsh Spartina anglica secretes up to $60 \%$ of the absorbed salt, while Glaux maritima can secrete just $20 \%$ of the total salt (Dassanayake and Larkin 2017). Mesembryanthemum crystallinum (ice plant) is considered a model for salt secretion through bladders. EBCs in ice plant contribute to salt sequestration and are also reservoirs of water, inorganic and organic compounds. Following salt stress, EBCs of ice plant can sequester up to $30 \%$ of the total $\mathrm{Na}^{+}$and $\mathrm{Cl}^{-}$in the leaves (Agarie et al. 2007).

The reports of EBCs as salt dumps in quinoa are based on the indirect estimation of the ion content in these structures by comparing ion contents of brushed and un-brushed leaves. In Chapter 2 we directly measured the ion content in EBCs from young leaves. From the total amount of ions in the leaves, EBCs accumulated $5 \%$ of $\mathrm{Na}^{+}, 6 \%$ of $\mathrm{Cl}^{-}$and $15 \%$ of $\mathrm{K}^{+}$. Based on these measurements, storage of salts in EBCs is not likely to contribute much to reducing $\mathrm{Na}^{+}$and $\mathrm{Cl}^{-}$ levels in the leaves as has been suggested, at least not in the genotypes included in this thesis. Kiana-Pouya et al (2017) reported that removing the EBCs in quinoa resulted in a salt-sensitive phenotype, which would indicate that EBCs are important for salt tolerance. Based on our results, we cannot exclude a possible contribution of EBCs to the salt tolerance of quinoa, but at least in our conditions an important role for EBCs in ion homeostasis seems unlikely. Other functions than salt dumps have been postulated for these cells. They could be water reservoirs and function as a secondary epidermis; this will be beneficial for the water balance in the leaves 
allowing the opening of stomata while minimizing water loss through cuticular evaporation. It has also been proposed that EBCs accumulate several compounds such as malate, flavonoids, betacyanin, pinitol, inositol and $\mathrm{Ca}^{2+}$ oxalate that can participate in several roles such as UV protection, stress signalling and ROS detoxification (Shabala and Munns 2017).

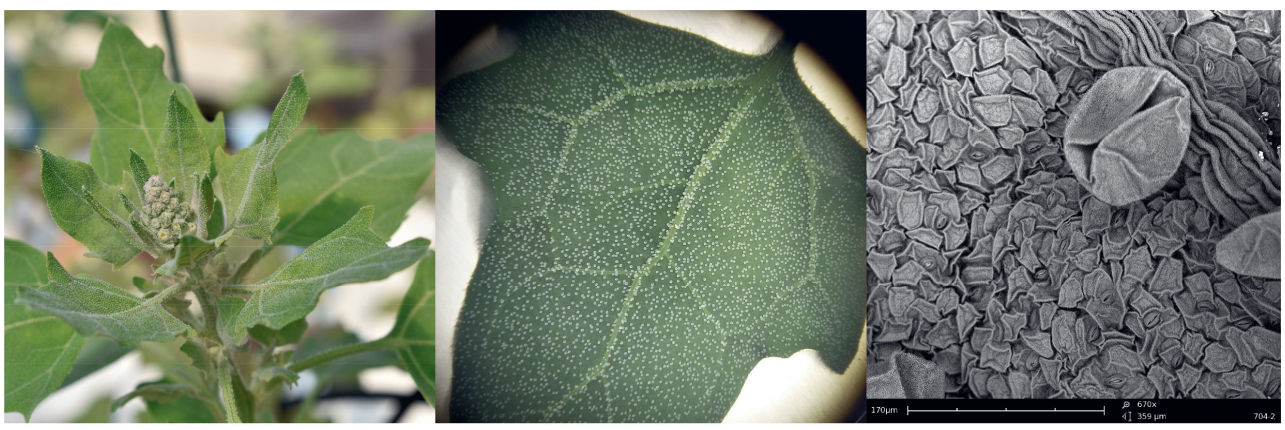

Figure 2. Epidermal bladder cells (EBCs) in quinoa in young tissues (left), under a light microscope (middle), and under a scanning electron microscope (right).

\subsection{Physiological adaptations to increase resource use efficiency}

The abundance of ions in salinized soils reduces water availability for roots. Ultimately, the adaptations in ion homeostasis of the plants discussed above contribute to salt tolerance and enable growth under stress conditions as long as they facilitate water uptake and balance within plant tissues. In this section we examine additional physiological features in quinoa related to the dynamics of plant water status and its relation to plant growth.

When water availability in the root media decreases and the water supply to transpiring leaves is insufficient, a common response of plants is to close their stomata to avoid loss of turgor, which leads to a decline in transpiration (Vadez et al. 2014). Moderate salt stress reduced the average stomatal conductance ( $g s$ ) in quinoa by $35 \%$ (Chapters 4, 5, 6), while under high salinity, gs was reduced by 60 $\%$ (Chapter 2, 4). Stomatal closure also limits $\mathrm{CO}_{2}$ uptake, which is why it constitutes a major limitation to photosynthesis under salinity (Chaves et al. 2011). However, the impact of stomatal closure on $\mathrm{CO}_{2}$ conductance is less than for water due to differences in the size and diffusion properties of these molecules (Condon et al. 2004). As a result, closing stomata up to a certain threshold can reduce water loss while maintaining sufficient $\mathrm{CO}_{2}$ uptake to sustain biomass gain (Peleg et al. 2011). To further understand the balance between water loss and carbon gain in quinoa, we used a high-resolution phenotyping platform (Plantarray ${ }^{\circledR}$ ) in Chapter 4 and analysed how water relations are affected by salt stress. 
Salinity strongly increased the water use efficiency (WUE) of quinoa plants. At the whole-plant scale, WUE is the ratio of biomass accumulation to water use over a period of time. An increased WUE has the potential to support greater carbon gain and productivity relative to the amount of water used by the crop. However, an increased WUE driven by lower water use is generally associated with conservative growth and can be an undesirable trait for productivity (Leakey et al. 2019). It has been pointed out that the benefits of an increased WUE are higher under severe stress, whereas under moderate stress it can be associated with too high yield penalties (Vadez et al. 2014). WUE is influenced by many traits including photosynthesis, stomatal and mesophyll conductance ( $\mathrm{gm}$ ), canopy structure as well as environmental factors such as water availability in the soil, temperature, atmospheric vapour-pressure deficit (VPD) (Leakey et al. 2019). Below we discuss different traits in quinoa that influence WUE and that might be modulated to increase WUE while simultaneously achieve higher productivity under salinity.

A reduction in gs reduces the loss of water through stomata, but plant adjustments in the regulation of stomatal closure and sensitivity to environmental changes can minimize the drawbacks from this reduction. An example is the sensitivity of stomata to VPD; closing stomata when VPD is high (midday) and keep stomata open at the beginning of the morning when VPD is low favours $\mathrm{CO}_{2}$ intake and limiting water loss (Sinclair 2018). Variations in stomata behaviour throughout the day and influences of the environmental conditions were more accentuated under salinity in quinoa plants than under control conditions (Chapter 4, figure 6). As a result, WUE under control conditions was stable during the day, but fluctuated under salinity due to the diurnal control of stomatal opening. How much water might be saved by quinoa plants in the long term by the diurnal control of stomatal opening remains to be estimated. We also observed a spatial control of stomatal opening under salinity. The gs reduction under salinity compared to control conditions was higher on the adaxial than the abaxial side of the leaves, possibly because the lower side of the leaves is less prone to water loss due to evaporation. The differential regulation of adaxial vs abaxial $g s$ under salinity has not been reported before and may contribute considerably to improved WUE. In addition, the regulation of the adaxial/abaxial gs ratio evidences the limitations of standard porometer measurements of $g s$ on only one side of the leaves. In summary, quinoa appears to have an adapted stomatal behaviour to maximize $\mathrm{CO}_{2}$ intake while preventing water loss. Genotypic differences and plasticity for these regulations might be additional strategies to improve quinoa productivity under salinity. 
WUE is also influenced by anatomical adaptations in the leaves. WUE is negatively correlated with specific leaf area (SLA): genotypes with thicker leaves show a higher WUE (Vadez et al. 2014). SLA can be used as a proxy for the carboxylation efficiency of the mesophyll; higher chlorophyll content from more packed mesophyll cells (lower SLA) leads to more active removal of intercellular $\mathrm{CO}_{2}$ (Ci) from the stomatal chamber, thus reducing the intercellular/ atmospheric $\mathrm{CO}_{2}$ (Ci/Ca) ratio and increasing WUE (Vadez et al. 2014). In Chapters 2 and 4 we established that the most-affected growth parameter at high salinity in quinoa is SLA. Under moderate salinity, genotypes with lower SLA and relatively high gs show a higher salt tolerance index (STI); the genotypes had a slightly lower surface area for water loss and possibly a higher $\mathrm{gm} / \mathrm{gs}$, both favourable traits to grow under salinity. QTLs for SLA have been considered to be contributing to WUE in several species (Vadez et al. 2014). It will be interesting to explore if the QTLs for SLA found in Chapter 5 under salinity point to genetic determinants of anatomical changes that influence water management in quinoa.

A decrease in SLA can also negatively affect WUE by limiting mesophyll conductance $(\mathrm{gm})$ (Flexas et al. 2008; Moshelion et al. 2015). However, this will depend on the anatomical changes linked to the reduction of SLA. For example, a thicker mesophyll (more layers and packed cells) will increase $\mathrm{gm}$ and positively affect WUE, while a lowered SLA due to the thickening of mesophyll cell walls will decrease gm and negatively affect WUE (Ouyang et al. 2017). Our exploration of the remodelling of quinoa cell walls under salinity (Chapter 3 ) did not point to the thickening of cell walls through lignification; on the contrary, the changes in the composition of the cell walls point to a more flexible and hydrated structure that may possibly enhance $\mathrm{gm}$. Understanding the structural changes that influence $\mathrm{gm}$ and possibly other physiological adaptations related to this trait (such as the expression and activity of aquaporins) (Ouyang et al. 2017) will help to be able to increase the $\mathrm{gm} / \mathrm{gs}$ ratio, which has been proposed as cost-efficient way to improve and breed for WUE (Condon 2020).

\subsection{Plant economics and salt tolerance}

All the quinoa genotypes examined in this thesis displayed a relatively high level of salt tolerance compared to other food crops. However, clear differences were found in individual genotypes for salt tolerance traits that affected their performance. Among the varieties, Pasto was noticeably different from the others in many of the measured traits. Pasto had the lowest $\left[\mathrm{Na}^{+}\right]$and $\left[\mathrm{Cl}^{-}\right]$in the leaves and the highest $\left[\mathrm{K}^{+}\right]$. In addition, it had the highest reduction in transpiration and the lowest growth 
rate, which was mostly associated with a decreased SLA. These traits point to a "conservative growth" strategy aimed at survival that is reflected in reduced growth and a higher investment in metabolically expensive stress tolerance traits $\left(\mathrm{K}^{+}\right.$ retention, cell wall elasticity). In other varieties like selRiobamba, Atlas and Red Carina transpiration was less reduced by saline conditions, $\mathrm{Na}^{+}$and $\mathrm{Cl}^{-}$accumulated to higher levels in the shoots and $\left[\mathrm{K}^{+}\right]$in leaves was maintained, but did not increase as much as in Pasto. These varieties appeared to follow an "acquisitive growth" strategy aimed at continued growth especially under moderate salt stress (Figure 3A). The relation between allocation of resources, functional traits and stress tolerance has been extensively studied (Balachowski and Volaire 2018; Reich et al. 2003). Fast growers or "acquisitive-growth" plants are normally more productive under moderate stress, but under prolonged or severe stress they might exhaust the limitedly available resources, risking plant failure and death. On the other hand, slow growers or "conservative growth" plants are typically more penalized under moderate stress, but better survivors under more severe conditions.

Quinoa is characterized by its rich genetic diversity (Ruiz et al. 2014). In this thesis we explored European commercial varieties, mapping populations resulting from crosses of these varieties and a collection of 22 genotypes of the Inter-Andean valley ecotype collected from Ecuador. The Ecuadorian material showed interesting properties for several agronomical traits (seed yield, yield stability, thousand seed weight (TSW)) and high salt tolerance similar to the European varieties. We also found genetic variation for salt tolerance traits, for instance with respect to the energy allocated to growth or tolerance adaptations. For this material, the best performers under moderate salinity also showed an "acquisitive-growth: higher transpiration and higher $\left[\mathrm{Na}^{+}\right]$and $\left[\mathrm{Cl}^{-}\right]$in leaves. It will be interesting to see whether the most salt tolerant quinoa genotypes reported in literature (e.g. originated from the Salares of Bolivia) (Hinojosa et al. 2018) contribute with different traits or balance of resources than the material evaluated in this thesis.

Plants growing in saline conditions experience major changes in energy metabolism, many of them associated with the strategy they use to distribute resources devoted to growth and the mitigation of stress (Sanders 2020). Salt tolerance can be defined in terms of energy consumption by the plants (Munns and Gilliham 2015; Zörb et al. 2019). Plant resources (carbon and energy) can be allocated to two main purposes: growth and maintenance (Amthor 2000). The allocation of photosynthates is strongly dependent on plant species, developmental stage and environmental conditions, but a conservative estimate for allocation to maintenance respiration of 45 to $60 \%$ was proposed (Amthor 2000). For the purpose 
of this analysis we will assume that under control conditions $50 \%$ of the available resources is allocated to maintenance respiration and the remaining $50 \%$ to growth. Based on the reduction in the radiation use efficiency (RUE) estimated in Chapter 4 we can calculate the reduction in the percentage of assimilates allocated to growth due to salinity; consequently, these resources might be invested in specific adaptations to salt stress (Figure 3B). Even though this analysis is a simplified alternative to examining the cost of salt tolerance that was proposed before (Munns and Gilliham 2015; Zörb et al. 2019), it allows a comparison of the amount of assimilates invested by different genotypes for salt tolerance. It also allows to compare the growth responses of "conservative" and "acquisitive" backgrounds. According to this estimation, under moderate salinity, Pasto allocated $20 \%$ less assimilates to growth that were used for salt tolerance, while selRiobamba allocated just $5 \%$ less assimilates to growth. Under high salinity, Pasto allocated $35 \%$ less assimilates to growth, and selRiobamba $30 \%$. From a crop-productivity perspective, "acquisitive-growth" varieties will be preferred since they allocate more assimilates into biomass and seed yield. However, "acquisitive-growth" can be risky if the stress becomes more severe or prolonged, which would possibly exhaust all the available resources for the plant and promote plant failure. It is also interesting to point out that under field-like conditions (Chapter 5) Pasto had higher salt tolerance than "acquisitive" varieties like Red Carina or Atlas. Under moderate salt stress and field conditions, the "energy-expensive" tolerance traits use by Pasto could be compensated by other parameters, such as a higher harvest index and TSW than the other varieties under salinity. The interesting conclusion from this comparison is that flexibility or plasticity in plant metabolism and functional traits in quinoa can provide the most cost-effective adaptations to perform well in saline environments (See section 2). 


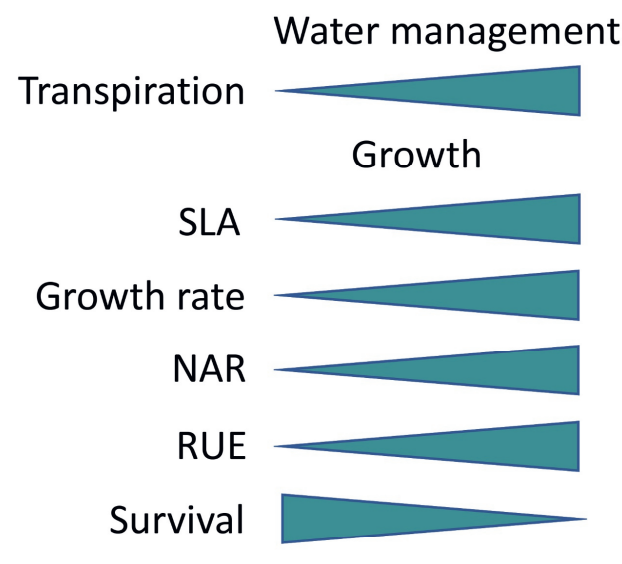

\title{
Ion/nutrient balance
}

$\left[\mathrm{NO}_{3}^{-}\right]$, $\left[\mathrm{K}^{+}\right]$in leaves

\author{
$\left[\mathrm{Cl}^{-}\right],\left[\mathrm{Na}^{+}\right]$in leaves
}

\section{Plant development}

Days to flowering

\section{Days to maturity}

B
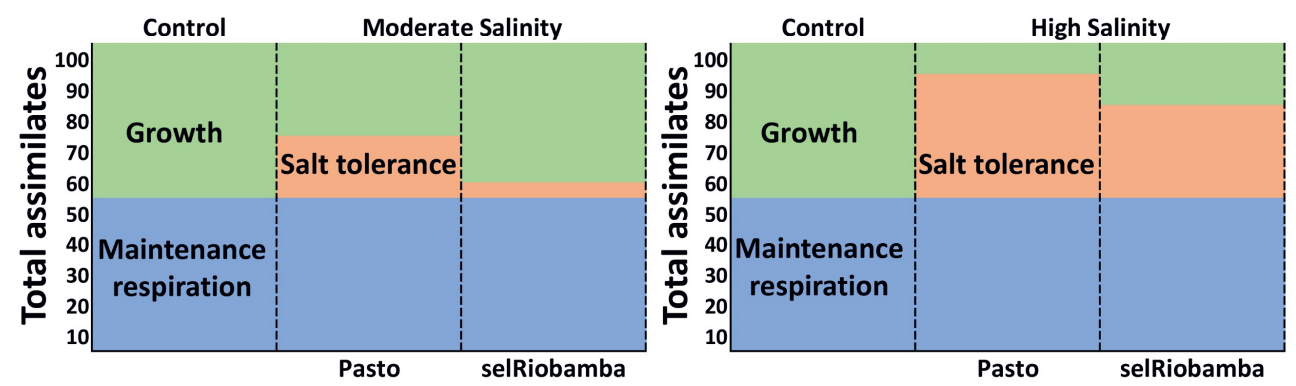

Figure 3. A) Comparison of growth strategies of two quinoa varieties (Pasto and selRiobamba). The thickness of the arrows indicates the differences in functional traits between "conservative" and "acquisitive" genotypes under salinity. B) Assimilates distribution in quinoa under control, moderate and high salt stress. The reduction in RUE under salinity was used to calculate the amount of assimilates that are allocated into salt tolerance (in orange) instead of growth (in green). Based on the estimations from (Amthor 2000), it was assumed that $50 \%$ of assimilates are allocated into maintenance respiration under control conditions. 


\section{Part II: Prospects of salt tolerance research: the role of quinoa}

The Amaranthaceae family includes a remarkably high number of halophytes, and several species from this family, for instance Suaeda and Salicornia have been explored as models to study the salt tolerance response of halophytes (Song and Wang 2014). While research in these species can provide novel insights into adaptations of plants to extreme environments, it is less likely that these mechanisms can be directly translated to crops; the low growth rates of most obligatory halophytes is not compatible with crop productivity (Cheeseman 2015). Quinoa, however, is a good alternative. The main advantage of using quinoa to understand salt tolerance in plants is its ability to withstand a broad range of salinity levels: it is considered a facultative halophyte. With some exceptions (Ruiz et al. $2016 \mathrm{~b}$ ), most of the quinoa genotypes grow better in the absence of salt (the main difference with obligatory halophytes). This means that is has adaptive mechanisms that can be activated to withstand salinity rather than constitutive salt tolerance processes in their metabolism that need to be adapted to non-saline conditions. In addition, by comparing the growth of quinoa at different levels of stress, we can differentiate cost-effective adaptations and traits that favour growth under mild stress conditions from conservative or survival adaptations that support survival under severe stress at the cost of growth. This approach could also facilitate the calculation of the energy costs of salt tolerance, an important topic in the current research of salt tolerance (Munns et al. 2020a).

Besides utilizing alternative crop species as models for tolerance mechanisms, salt tolerance research has benefited and will continue to benefit from biotechnological tools, automatized phenotyping technologies and the integration of plant traits into mechanistic crop models. For instance, crop models can help to understand the interaction of $g s, g m, S L A$ and the contribution of this interaction to an increased WUE and water productivity. The input data for such models might be obtained from high-throughput automatized phenotypic technologies based on hyperspectral reflectance, thermal and chlorophyll fluorescence, gas exchange, magnetic resonance imaging (Pieruschka and Schurr 2019). In Chapter 4 we used one of these platforms (Plantarray $囚$ ) and demonstrated the advantages of obtaining high temporal-resolution data that give insight in plant responses throughout the whole season. The integration of high-resolution data into crop models will set the pace for future salt tolerance research, and ideally will facilitate the construction of a plant ideotype for salt tolerance. Based on the knowledge acquired from this thesis, I present below a few features of a first draft on what I consider a quinoa ideotype will look like (Figure 4). 
- Ion exclusion: This ideotype will have a high rate of ion exclusion. Based on our measurements, at early stages of salt stress the exclusion rate will be similar to other tolerant plants ( $98 \%$ ), which indicates that quinoa is able to tolerate $\mathrm{Na}^{+}$and $\mathrm{Cl}^{-}$concentrations in tissues up to a certain level. The intake of ions during these weeks will support osmotic adjustment and growth in a cost-effective way. If the stress remains for longer periods and ion concentrations rise in the shoots, the plant will be flexible and fast in strengthening ion exclusion (>99\%). This will require additional energy but protect tissues from potential ionic damage.

- $\mathrm{K}^{+}$maintenance: The plant requires transporters that are highly selective for $\mathrm{K}^{+}$despite the abundance of $\mathrm{Na}^{+}$. In all the assessed genotypes the $\mathrm{K}^{+} / \mathrm{Na}^{+}$ was never lower than one, while in most plant species under saline conditions it drops to well below 1 . Shoot $\left[\mathrm{K}^{+}\right]$should follow $\left[\mathrm{Na}^{+}\right]$. In spite of the metabolic cost, if $\left[\mathrm{Na}^{+}\right]$increases, $\mathrm{K}^{+}$should be the main contributor to the tissue tolerance of the plant. Possibly, the ability of quinoa to raise $\left[\mathrm{K}^{+}\right]$ (especially when $\left[\mathrm{Na}^{+}\right]$rises) is the main "facultative" trait of this species.

- Decrease of specific leaf area (SLA): In this hypothetical phenotype, SLA should be negatively correlated to the severity of the stress. SLA reduction will be caused by a thicker mesophyll (more cell layers) and not by an increased amount of cell wall (lignification). This reduced SLA will have several benefits for the plant: increase in photosynthetic efficiency by a higher concentration of Rubisco and chlorophyll, increase in $\mathrm{gm}$, slight increase in succulence that might dilute ion concentrations, and decrease transpiration with less impact on gs, which will not compromise the cooling of the canopy.

- Responsive stomata: This plant requires a responsive control of stomatal opening to maximize water saving. Some of the responses assessed in this thesis have the highest peaks of gs in the morning and lowest $g s$ at the peaks of VPD, and a higher stomatal reduction in the adaxial than the abaxial side of the leaves. This responsive control of stomatal opening will contribute to a "smart" increase in WUE that will not be associated with a lower growth of plants.

- Increase in elasticity and hydration capacity of cell walls: This adaptation will create plastic cell walls capable to protect cell integrity in spite of changes in turgor pressure. In this way, cell wall remodelling under stress will not be just a consequence of the stress, but an adaptive response to a changing environment. 
- Epidermal bladder cells: Based on our results, it is unlikely that EBCs contribute to the ion homeostasis of quinoa. However, this anatomical feature might contribute to reduce cuticular evaporation from leaves.

- Redistribution of assimilates: Changes in the allocation of assimilates in the plant might play a pivotal role for quinoa productivity under salinity. An increase in harvest index reduced the impact of salt in yield under field-like conditions. We also measured an increase in the biomass allocation into roots; the contribution of this trait to the tolerance of quinoa deserves further exploration.

- Growth plasticity: This plant might have the flexibility to switch from "acquisitive" to more "conservative" growth traits depending on the severity/ duration of the stress. Under short-term/ mild stress, "acquisitive" traits might be advantageous for crop productivity, while at high salinity, "conservative" traits seem to be essential for plant survival.

This described ideotype can be made or even improved with the help of the tools mentioned above: high-resolution phenotyping to discover more traits, biotechnological tools to discover the genetic determinants of these traits and mechanistic models to estimate the energy budgets they require. The successfulness of this hypothetical cultivar will rely on how it can balance energy allocated to grow and to mitigate stress.

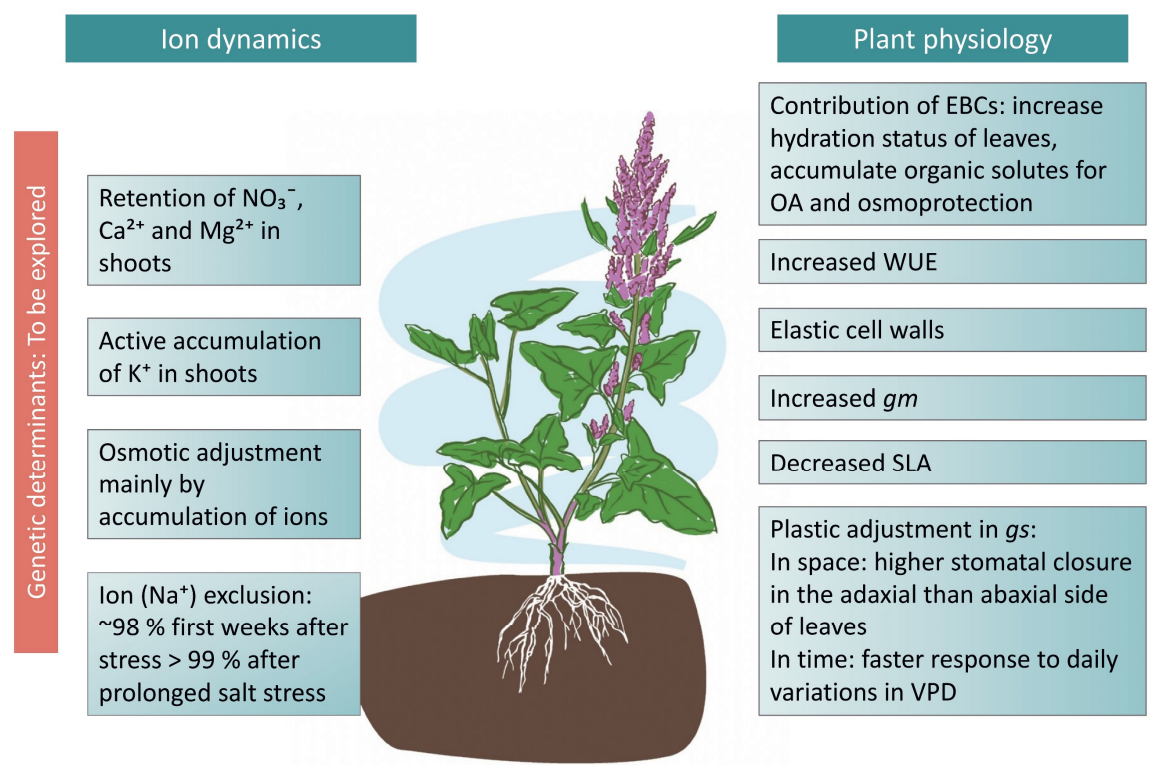

Figure 4. Representative ideotype for salt tolerance in quinoa based on the salt tolerance traits discussed in this thesis. 


\section{Part III: The future of quinoa as a consolidated staple crop}

Currently, the main targets in breeding programs of quinoa are increasing yield and yield stability to close the existing gap between potential and actual farm yields (López-Marqués et al. 2020). The worldwide average yield of quinoa is 2-2.5 ton/ha while the maximum potential yield has been estimated as high as $8-10$ ton/ha (Choukr-Allah et al. 2016). In our trials in the Netherlands we have obtained yields of 5 ton/ha and, under controlled conditions, we even measured yields of 12 ton/ha for some of the Ecuadorian genotypes (Chapter 6). The yield gap questions the competitiveness of quinoa with other crops, cereals for instance. Nevertheless, the high market value and worldwide demand make quinoa an attractive commodity. As an example, it was recently calculated that despite the low yield of quinoa in field trials in the Palouse region the United States (average 1.04 ton/ha), quinoa production was still profitable due to the price and position of the grain in the market (Wieme et al. 2020a).

As has been stated throughout this thesis, one of the biggest incentives to expand the cultivation of quinoa is its high resilience to abiotic stress, salinity being arguably the main one. An evaluation of wheat and maize production in the last 20 years calculated that abiotic stress (mainly drought) reduces wheat yield by $20 \%$ and maize by $40 \%$ (Daryanto et al. 2016). In these adverse conditions, quinoa can potentially compete with major crops. With quinoa being inherently highly salt tolerant, a relevant question is whether improving salt tolerance is a target worth exploring in quinoa breeding programs. I believe it certainly is. Recent studies show that salt tolerance varies significantly between quinoa genotypes (Jaikishun et al. 2019; Peterson and Murphy 2015b). Several field trials have examined the potential of quinoa to grow in areas affected by salinity in the Middle East and North Africa (MENA) and Central Asia, amongst others in Vietnam, China, Turkey, Algeria, Egypt, Iran, Iraq, Saudi Arabia, Lebanon, Mauritania, Sudan Yemen and Morocco (ChoukrAllah et al. 2016). The main conclusions of these trials are that quinoa yields are still low and unpredictable (1.2-1.4 ton/ha), and there is high variation in the performance of different varieties across locations. The identification and breeding of the bestyielding varieties with good local adaptation and high nutritional quality is crucial to move forward from experimental and field trials to a commercial production scale of quinoa in marginal environments. Our exploration of salt tolerance traits in two biparental populations (Chapter 5) showed that all the traits segregated in the populations, which means that desired alleles for these traits can be identified and incorporated in breeding programs through marker assisted selection, and improving salt tolerance in these varieties is possible. 
It is important to consider that marginal lands often possess a wide range of undesirable properties in addition to salinity (poor nutrition quality, dry spells, extreme temperatures, etc.) (Kang et al. 2013). The success of cultivating quinoa in these areas depends not only on the adaptability of salt tolerant genotypes but also on screening and breeding of very resilient varieties capable of adapting to combinations of these stress factors. Research on synergistic responses to these environmental factors is being developed (Hinojosa et al. 2018) and will determine whether quinoa can flourish in marginal areas. The screening and selection of the most salt tolerant varieties will also broaden the irrigation options for quinoa cultivation. Until now, a deficit irrigation strategy is considered a valuable and sustainable production strategy in regions where intra-seasonal dry spells are occurring (Choukr-Allah et al. 2016). Based on our results we consider that irrigation with seawater would not be economically profitable; but certainly, cultivation of quinoa irrigated with brackish water in for instance saline deltas of the world may be possible. Another potential use of quinoa is as a rotation crop in dry or salinized areas in the United States and Europe (Wieme et al. 2020b). The adoption of quinoa as a rotation crop will depend on several factors, including the selection of locally adapted profitable varieties, and the improvement and automatization of agronomical practices. To sum up, salt tolerance is definitely a breeding target of quinoa, scientifically relevant, and a key determinant of the economic profitability of the cultivation of the crop around the globe.

Quinoa production has been increasing over the past few years. Around 2013, the so-called "quinoa boom" was characterized by a peak in its production, and fluctuating prices (Bedoya-Perales et al. 2018). However, in the last years, the market and the price have reached an equilibrium: the demand of the crop is still projected to grow but in a more gradual pattern (Angeli et al. 2020). The projected growth of quinoa cultivation for the future decades has two main drivers. The first one, mentioned above, the potential of quinoa as an alternative to grow in marginal lands where food insecurity and malnutrition are socio-economic ongoing problems. In addition, the current consumer requirements put quinoa in a very strategic position to succeed. The current market is characterized by consumer awareness and demand of healthy and environmentally friendly products. Quinoa fits well in these contemporary requirements: the expansion of "plant-based" diets as a strategy to reduce land clearing by animal farming, the re-valorisation of plant-origin proteins, the demand for functional products as well as gluten-free products, etc. There is a general consensus that these demands will continue to rise (Angeli et al. 2020), laying the foundations for a bright future for quinoa. 
The drivers for the expansion of quinoa are clear. Now, how can breeding contribute to strengthen the expansion of the crop? Quinoa's research will benefit from the development of tools to assist the breeding of the crop at all levels. From the sequence of the first quinoa genome (Jarvis et al. 2017), several tools are being developed to assist breeding programs. In this thesis we implemented Allegro genotyping ${ }^{\circledR}$, a novel technology to identify single nucleotide polymorphisms (SNPs) in a cost-effective manner (Scaglione et al. 2019). The markers as well as the genome sequence are part of a molecular toolbox that will facilitate marker-assisted quinoa breeding. Other breeding tools such as experimental populations for QTL mapping, TILLING or mutagenesis protocols for trait detection, or the development of strategies to shorten the seed generation time will further accelerate the improvement of quinoa and contribute to close the existing yield gap. The implementation of phenotyping techniques will also facilitate the screening of big populations and the identification of desirable traits. The ultimate goal of breeding programs is the development of varieties that combine high yield potential, tolerance to abiotic and biotic stresses, adaptability to diverse agroclimatic zones and suitable grain quality for food and industry.

The use of quinoa is connected to the historical and cultural cultivation by indigenous communities in the Andean region. It has been argued that the increasing production of quinoa in different areas in the world could threaten the growers from native production areas, particularly smallholder farmers (Angeli et al. 2020). However, considering the growing demand on the crop, this could rather be a unique opportunity to strengthen the production systems of small growers and increase their competitiveness in this growing market. As an example, the development of new varieties with improved productivity for the harsh landscapes of the Andes (including salinized lands) could increase the revenue of small growers. The development of international consortia, and participatory breeding programs are potential strategies to protect the rural economies of growers in local communities and, simultaneously, provide access to valuable germplasm with the potential to face food insecurity in other areas of the world (Murphy et al. 2016; Nunez de Arco 2015). It is my opinion that protecting the smallholder farmers in the Andes is a responsibility of all the stakeholders involved in quinoa production chains, and this can already be achieved by the cooperation between local and international research institutes, corporate participation and the willingness to do so from local governments and communities. Quinoa breeding and quinoa research have the potential to benefit all the involved actors, which will consolidate the transition of this crop from a forgotten pseudocereal in the Andes to a well-established food staple commodity. 



\section{References}

Abedon BG, Hatfield RD, Tracy WF (2006) Cell Wall Composition in Juvenile and Adult Leaves of Maize (Zea mays L.). Journal of Agricultural and Food Chemistry 54 (11):3896-3900. doi:10.1021/jf052872w

Adolf V, Jacobsen S-E, Shabala S (2013) Salt tolerance mechanisms in quinoa (Chenopodium quinoa Willd.). Environmental and Experimental Botany 92:43-54. doi:10.1016/j.envexpbot.2012.07.004

Adolf VI, Shabala S, Andersen MN, Razzaghi F, Jacobsen SE (2012) Varietal differences of quinoa's tolerance to saline conditions. Plant and Soil 357 (1):117-129. doi:10.1007/s11104-012-1133-7

Agarie S, Shimoda T, Shimizu Y, Baumann K, Sunagawa H, Kondo A, Ueno O, Nakahara T, Nose A, Cushman JC (2007) Salt tolerance, salt accumulation, and ionic homeostasis in an epidermal bladder-cell-less mutant of the common ice plant Mesembryanthemum crystallinum. Journal of Experimental Botany 58 (8):1957-1967. doi:10.1093/jxb/erm057

Ahmad I, Maathuis FJM (2014) Cellular and tissue distribution of potassium: Physiological relevance, mechanisms and regulation. Journal of Plant Physiology 171 (9):708-714. doi:https://doi.org/10.1016/j.jplph.2013.10.016 Alqahtani M, Roy SJ, Tester M (2019) Increasing Salinity Tolerance of Crops. In: Savin R, Slafer GA (eds) Crop Science. Springer New York, New York, NY, pp 245-267. doi:10.1007/978-1-4939-8621-7_429

Amthor JS (2000) The McCree-de Wit-Penning de Vries-Thornley Respiration Paradigms: 30 Years Later. Annals of Botany 86 (1):1-20. doi:10.1006/anbo.2000.1175

An P, Li X, Zheng Y, Matsuura A, Abe J, Eneji AE, Tanimoto E, Inanaga S (2014) Effects of $\mathrm{NaCl}$ on Root Growth and Cell Wall Composition of Two Soya bean Cultivars with Contrasting Salt Tolerance. Journal of Agronomy and Crop Science 200 (3):212-218. doi:10.1111/jac.12060

Angeli V, Miguel Silva P, Crispim Massuela D, Khan MW, Hamar A, Khajehei F, Graeff-Hönninger S, Piatti C (2020) Quinoa (Chenopodium quinoa Willd.): An Overview of the Potentials of the "Golden Grain" and Socio-Economic and Environmental Aspects of Its Cultivation and Marketization. Foods 9 (2). doi:10.3390/foods9020216

Aquino RS, Grativol C, Mourão PAS (2011) Rising from the sea: correlations between sulfated polysaccharides and salinity in plants. PLoS One 6 (4):e18862-e18862. doi:10.1371/journal.pone.0018862

Ashraf M, Khanum A (1997) Relationship Between Ion Accumulation and Growth in Two Spring Wheat Lines Differing in Salt Tolerance at Different Growth Stages. Journal of Agronomy and Crop Science 178 (1):39-51. doi:10.1111/j.1439-037X.1997.tb00349.x

Asif MA, Schilling RK, Tilbrook J, Brien C, Dowling K, Rabie H, Short L, Trittermann C, Garcia A, Barrett-Lennard EG, Berger B, Mather DE, Gilliham M, Fleury D, Tester M, Roy SJ, Pearson AS (2018) Mapping of novel salt tolerance QTL in an Excalibur $\times$ Kukri doubled haploid wheat population. Theor Appl Genet 131 (10):2179-2196. doi:10.1007/s00122-018-3146-y

Balachowski JA, Volaire FA (2018) Implications of plant functional traits and drought survival strategies for ecological restoration. Journal of Applied Ecology 55 (2):631-640. doi:10.1111/1365-2664.12979 
Barkla BJ, Vera-Estrella R (2015) Single cell-type comparative metabolomics of epidermal bladder cells from the halophyte Mesembryanthemum crystallinum. Frontiers in Plant Science 6 (435). doi:10.3389/fpls.2015.00435

Bazile D, Fuentes F, Mujica A (2013) Historical perspectives and domestication of quinoa. In.

Bazile D, Jacobsen S-E, Verniau A (2016a) The Global Expansion of Quinoa: Trends and Limits. Frontiers in Plant Science 7 (622). doi:10.3389/fpls.2016.00622

Bazile D, Pulvento C, Verniau A, Al-Nusairi MS, Ba D, Breidy J, Hassan L, Mohammed MI, Mambetov O, Otambekova M, Sepahvand NA, Shams A, Souici D, Miri K, Padulosi S (2016b) Worldwide evaluations of quinoa: Preliminary results from post international year of quinoa FAO projects in nine countries. Frontiers in Plant Science 7. doi:10.3389/fpls.2016.00850

Bazile DB, F. (2015) The dynamics of the global expansion of quinoa growing in view of its high biodiversity. In: D. Bazile HD, Bertero, and C. Nieto (ed) State of the Art Report on Quinoa Around the World in 2013. FAO\&CIRAD, Roma, pp 42-55

Becker VI, Goessling JW, Duarte B, Caçador I, Liu F, Rosenqvist E, Jacobsen S-E (2017) Combined effects of soil salinity and high temperature on photosynthesis and growth of quinoa plants (Chenopodium quinoa). Functional Plant Biology 44 (7):665-678. doi:https://doi.org/10.1071/FP16370

Bedoya-Perales NS, Pumi G, Mujica A, Talamini E, Domingos Padula A (2018) Quinoa Expansion in Peru and Its Implications for Land Use Management. Sustainability 10 (2). doi:10.3390/su10020532

Ben Hamed K, Dabbous A, El Shaer H, Abdely C (2018) Salinity Responses and Adaptive Mechanisms in Halophytes and Their Exploitation for Producing Salinity Tolerant Crops. In: Kumar V, Wani SH, Suprasanna P, Tran L-SP (eds) Salinity Responses and Tolerance in Plants, Volume 2: Exploring RNAi, Genome Editing and Systems Biology. Springer International Publishing, Cham, pp 1-19. doi:10.1007/978-3-319-90318-7_1

Benito B, Haro R, Amtmann A, Cuin TA, Dreyer I (2014) The twins $\overline{\mathrm{K}}+$ and $\mathrm{Na}+$ in plants. Journal of Plant Physiology 171 (9):723-731. doi:https://doi.org/10.1016/j.jplph.2013.10.014

Blum A (2009) Effective use of water (EUW) and not water-use efficiency (WUE) is the target of crop yield improvement under drought stress. Field Crops Research 112 (2):119-123. doi:https://doi.org/10.1016/i.fcr.2009.03.009

Bolger AM, Lohse M, Usadel B (2014) Trimmomatic: a flexible trimmer for Illumina sequence data. Bioinformatics 30 (15):2114-2120. doi:10.1093/bioinformatics/btu170

Britto DT, Kronzucker HJ (2015) Sodium efflux in plant roots: What do we really know? Journal of Plant Physiology 186-187:1-12. doi:https://doi.org/10.1016/j.jplph.2015.08.002

Bromham L (2014) Macroevolutionary patterns of salt tolerance in angiosperms. Annals of Botany 115 (3):333-341. doi:10.1093/aob/mcu229

Buckley TN (2005) The control of stomata by water balance. New Phytologist 168 (2):275-292. doi:10.1111/j.1469-8137.2005.01543.x 
Burton RA, Gidley MJ, Fincher GB (2010) Heterogeneity in the chemistry, structure and function of plant cell walls. Nature Chemical Biology 6 (10):724-732. doi:10.1038/nchembio.439

Byrt CS, Munns R, Burton RA, Gilliham M, Wege S (2018) Root cell wall solutions for crop plants in saline soils. Plant Science 269:47-55. doi:https://doi.org/10.1016/j.plantsci.2017.12.012

Cabane M, Afif D, Hawkins S (2012) Chapter 7 - Lignins and Abiotic Stresses. In: Jouanin L, Lapierre C (eds) Advances in Botanical Research, vol 61. Academic Press, pp 219-262. doi:https://doi.org/10.1016/B978-0-12416023-1.00007-0

Cabrera-Bosquet L, Fournier C, Brichet N, Welcker C, Suard B, Tardieu F (2016) High-throughput estimation of incident light, light interception and radiationuse efficiency of thousands of plants in a phenotyping platform. New Phytologist 212 (1):269-281. doi:10.1111/nph.14027

Caffall KH, Mohnen D (2009) The structure, function, and biosynthesis of plant cell wall pectic polysaccharides. Carbohydrate Research 344 (14):1879-1900. doi:https://doi.org/10.1016/j.carres.2009.05.021

Cankar K, Kortstee A, Toonen MA, Wolters-Arts M, Houbein R, Mariani C, Ulvskov P, Jorgensen B, Schols HA, Visser RG, Trindade LM (2014) Pectic arabinan side chains are essential for pollen cell wall integrity during pollen development. Plant Biotechnol J 12 (4):492-502. doi:10.1111/pbi.12156

Chaves MM, Flexas J, Pinheiro C (2009) Photosynthesis under drought and salt stress: regulation mechanisms from whole plant to cell. Annals of botany 103 (4):551-560. doi:10.1093/aob/mcn125

Chaves MM, Miguel Costa J, Madeira Saibo NJ (2011) Chapter 3 - Recent Advances in Photosynthesis Under Drought and Salinity. In: Turkan I (ed) Advances in Botanical Research, vol 57. Academic Press, pp 49-104. doi:https://doi.org/10.1016/B978-0-12-387692-8.00003-5

Cheeseman JM (2015) The evolution of halophytes, glycophytes and crops, and its implications for food security under saline conditions. New Phytologist 206 (2):557-570. doi:10.1111/nph.13217

Cheng A (2018) Review: Shaping a sustainable food future by rediscovering longforgotten ancient grains. Plant Science 269:136-142. doi:10.1016/j.plantsci.2018.01.018

Choukr-Allah R, Rao NK, Hirich A, Shahid M, Alshankiti A, Toderich K, Gill S, Butt KUR (2016) Quinoa for Marginal Environments: Toward Future Food and Nutritional Security in MENA and Central Asia Regions. Frontiers in Plant Science 7 (346). doi:10.3389/fpls.2016.00346

Christiansen JL, Jacobsen SE, Jørgensen ST (2010) Photoperiodic effect on flowering and seed development in quinoa (Chenopodium quinoa willd.). Acta Agriculturae Scandinavica Section B: Soil and Plant Science 60 (6):539-544. doi:10.1080/09064710903295184

Colmer TD, Munns R, Flowers TJ (2005) Improving salt tolerance of wheat and barley: Future prospects. Australian Journal of Experimental Agriculture 45 (11):1425-1443. doi:10.1071/EA04162

Condon AG (2020) Drying times: plant traits to improve crop water use efficiency and yield. Journal of Experimental Botany 71 (7):2239-2252.

doi:10.1093/jxb/eraa002 
Condon AG, Richards RA, Rebetzke GJ, Farquhar GD (2004) Breeding for high water-use efficiency. Journal of Experimental Botany 55 (407):2447-2460. doi:10.1093/jxb/erh277

Corrêa-Ferreira ML, Viudes EB, de Magalhães PM, Paixão de Santana Filho A, Sassaki GL, Pacheco AC, de Oliveira Petkowicz CL (2019) Changes in the composition and structure of cell wall polysaccharides from Artemisia annua in response to salt stress. Carbohydrate Research 483:107753. doi:https://doi.org/10.1016/i.carres.2019.107753

Cosgrove DJ (2005) Growth of the plant cell wall. Nature Reviews Molecular Cell Biology 6 (11):850-861. doi:10.1038/nrm1746

Cosgrove DJ (2015) Plant cell wall extensibility: connecting plant cell growth with cell wall structure, mechanics, and the action of wall-modifying enzymes. Journal of Experimental Botany 67 (2):463-476. doi:10.1093/jxb/erv511

Cuartero J, Bolarín MC, Asíns MJ, Moreno V (2006) Increasing salt tolerance in the tomato. Journal of Experimental Botany 57 (5):1045-1058. doi:10.1093/jxb/erj102

Daliakopoulos IN, Tsanis IK, Koutroulis A, Kourgialas NN, Varouchakis AE, Karatzas GP, Ritsema CJ (2016) The threat of soil salinity: A European scale review. Science of the Total Environment 573:727-739. doi:10.1016/j.scitotenv.2016.08.177

Daryanto S, Wang L, Jacinthe P-A (2016) Global Synthesis of Drought Effects on Maize and Wheat Production. PLoS One 11 (5):e0156362. doi:10.1371/journal.pone. 0156362

Dassanayake M, Larkin JC (2017) Making Plants Break a Sweat: the Structure, Function, and Evolution of Plant Salt Glands. Frontiers in Plant Science 8 (406). doi:10.3389/fpls.2017.00406

de Lima RB, dos Santos TB, Vieira LGE, de Lourdes Lúcio Ferrarese M, Ferrarese-Filho O, Donatti L, Boeger MRT, de Oliveira Petkowicz CL (2014) Salt stress alters the cell wall polysaccharides and anatomy of coffee (Coffea arabica L.) leaf cells. Carbohydrate Polymers 112:686-694. doi:https://doi.org/10.1016/j.carbpol.2014.06.042

De Vries FWTP (1975) The Cost of Maintenance Processes in Plant Cells. Annals of Botany 39 (1):77-92. doi:10.1093/oxfordjournals.aob.a084919

Deinlein U, Stephan AB, Horie T, Luo W, Xu G, Schroeder JI (2014) Plant salttolerance mechanisms. Trends in Plant Science 19 (6):371-379. doi:10.1016/j.tplants.2014.02.001

Doblin MS, Pettolino F, Bacic A (2010) Plant cell walls: the skeleton of the plant world. Functional Plant Biology 37 (5):357-381. doi:https://doi.org/10.1071/FP09279

Doyle J (1991) DNA Protocols for Plants. In: Hewitt GM, Johnston AWB, Young JPW (eds) Molecular Techniques in Taxonomy. Springer Berlin Heidelberg, Berlin, Heidelberg, pp 283-293. doi:10.1007/978-3-642-839627_18

Endler A, Kesten C, Schneider R, Zhang Y, Ivakov A, Froehlich A, Funke N, Persson S (2015) A Mechanism for Sustained Cellulose Synthesis during Salt Stress. Cell 162 (6):1353-1364. doi:https://doi.org/10.1016/j.cell.2015.08.028 
Fahlgren N, Gehan MA, Baxter I (2015) Lights, camera, action: High-throughput plant phenotyping is ready for a close-up. Current Opinion in Plant Biology 24:93-99. doi:10.1016/j.pbi.2015.02.006

Fan Y, Shabala S, Ma Y, Xu R, Zhou M (2015) Using QTL mapping to investigate the relationships between abiotic stress tolerance (drought and salinity) and agronomic and physiological traits. BMC Genomics 16 (1):43. doi:10.1186/s12864-015-1243-8

FAOSTAT Statistical Database (2020) http://www.fao.org/faostat/en/\#home.

Flexas J, Bota J, Loreto F, Cornic G, Sharkey TD (2004) Diffusive and Metabolic Limitations to Photosynthesis under Drought and Salinity in C3 Plants. Plant Biology 6 (3):269-279. doi:10.1055/s-2004-820867

Flexas J, Ribas-CarbÓ M, Diaz-Espejo A, GalmÉS J, Medrano H (2008) Mesophyll conductance to $\mathrm{CO} 2$ : current knowledge and future prospects. Plant, Cell \& Environment 31 (5):602-621. doi:10.1111/j.1365-3040.2007.01757.x

Flowers TJ (2004) Improving crop salt tolerance. J Exp Bot 55 (396):307-319. doi:10.1093/jxb/erh003

Flowers TJ, Colmer TD (2008) Salinity tolerance in halophytes. New Phytol 179 (4):945-963. doi:10.1111/j.1469-8137.2008.02531.x

Flowers TJ, Colmer TD (2015) Plant salt tolerance: adaptations in halophytes. Annals of Botany 115 (3):327-331. doi:10.1093/aob/mcu267

Flowers TJ, Galal HK, Bromham L (2010) Evolution of halophytes: multiple origins of salt tolerance in land plants. Functional Plant Biology 37 (7):604-612

Flowers TJ, Munns R, Colmer TD (2015) Sodium chloride toxicity and the cellular basis of salt tolerance in halophytes. Annals of botany 115 (3):419-431. doi:10.1093/aob/mcu217

Fuentes FF, Bazile D, Bhargava A, Martínez EA (2012) Implications of farmers' seed exchanges for on-farm conservation of quinoa, as revealed by its genetic diversity in Chile. Journal of Agricultural Science 150 (6):702-716. doi:10.1017/S0021859612000056

Furbank RT, Tester M (2011) Phenomics - technologies to relieve the phenotyping bottleneck. Trends in Plant Science 16 (12):635-644. doi:10.1016/j.tplants.2011.09.005

Gajdanowicz P, Michard E, Sandmann M, Rocha M, Corrêa LGG, Ramírez-Aguilar SJ, Gomez-Porras JL, González W, Thibaud J-B, van Dongen JT, Dreyer I (2011) Potassium ( $\mathrm{K}<$ sup $>+</$ sup $>$ ) gradients serve as a mobile energy source in plant vascular tissues. Proceedings of the National Academy of Sciences 108 (2):864-869. doi:10.1073/pnas.1009777108

Geissler N, Hussin S, El-Far MMM, Koyro HW (2015) Elevated atmospheric CO2 concentration leads to different salt resistance mechanisms in a C3 (Chenopodium quinoa) and a C4 (Atriplex nummularia) halophyte. Environmental and Experimental Botany 118:67-77. doi:10.1016/j.envexpbot.2015.06.003

Genc Y, Tester M, McDonald GK (2010) Calcium requirement of wheat in saline and non-saline conditions. Plant and Soil 327 (1):331-345. doi:10.1007/s11104-009-0057-3

Gilliham M, Able JA, Roy SJ (2017) Translating knowledge about abiotic stress tolerance to breeding programmes. The Plant Journal 90 (5):898-917. doi:10.1111/tpj.13456 
Glenn EP, Brown JJ, Blumwald E (1999) Salt Tolerance and Crop Potential of Halophytes. Critical Reviews in Plant Sciences 18 (2):227-255. doi:10.1080/07352689991309207

Gomez-Pando LR, Aguilar-Castellanos E, Ibañez-Tremolada M (2019) Quinoa (Chenopodium quinoa Willd.) Breeding. In: Al-Khayri JM, Jain SM, Johnson DV (eds) Advances in Plant Breeding Strategies: Cereals: Volume 5. Springer International Publishing, Cham, pp 259-316. doi:10.1007/978-3-030-23108-8 7

Gosa SC, Lupo Y, Moshelion M (2019) Quantitative and comparative analysis of whole-plant performance for functional physiological traits phenotyping: New tools to support pre-breeding and plant stress physiology studies. Plant Science 282:49-59. doi:https://doi.org/10.1016/i.plantsci.2018.05.008

Hackl H, Baresel JP, Mistele B, Hu Y, Schmidhalter U (2012) A Comparison of Plant Temperatures as Measured by Thermal Imaging and Infrared Thermometry. Journal of Agronomy and Crop Science 198 (6):415-429. doi:10.1111/j.1439-037X.2012.00512.x

Hariadi Y, Marandon K, Tian Y, Jacobsen SE, Shabala S (2011) Ionic and osmotic relations in quinoa (Chenopodium quinoa Willd.) plants grown at various salinity levels. Journal of Experimental Botany 62 (1):185-193. doi:10.1093/jxb/erq257

Harris BN, Sadras VO, Tester M (2010) A water-centred framework to assess the effects of salinity on the growth and yield of wheat and barley. Plant and Soil 336 (1):377-389. doi:10.1007/s11104-010-0489-9

Hasegawa PM, Bressan RA, Zhu J-K, Bohnert HJ (2000) PLANT CELLULAR AND MOLECULAR RESPONSES TO HIGH SALINITY. Annual Review of Plant Physiology and Plant Molecular Biology 51 (1):463-499. doi:10.1146/annurev.arplant.51.1.463

Hedrich R, Shabala S (2018) Stomata in a saline world. Current Opinion in Plant Biology 46:87-95. doi:https://doi.org/10.1016/j.pbi.2018.07.015

Henderson SW, Baumann U, Blackmore DH, Walker AR, Walker RR, Gilliham M (2014) Shoot chloride exclusion and salt tolerance in grapevine is associated with differential ion transporter expression in roots. BMC Plant Biology 14 (1):273. doi:10.1186/s12870-014-0273-8

Hinojosa L, González AJ, Barrios-Masias HF, Fuentes F, Murphy MK (2018) Quinoa Abiotic Stress Responses: A Review. Plants 7 (4). doi:10.3390/plants7040106

Hirich A, Jelloul A, Choukr-Allah R, Jacobsen S-E (2014) Saline Water Irrigation of Quinoa and Chickpea: Seedling Rate, Stomatal Conductance and Yield Responses. Journal of Agronomy and Crop Science 200 (5):378-389. doi:doi:10.1111/jac.12072

Houston K, Tucker MR, Chowdhury J, Shirley N, Little A (2016) The Plant Cell Wall: A Complex and Dynamic Structure As Revealed by the Responses of Genes under Stress Conditions. Frontiers in Plant Science 7 (984). doi:10.3389/fpls.2016.00984

Hussain MI, Al- Dakheel AJ, Reigosa MJ (2018) Genotypic differences in agrophysiological, biochemical and isotopic responses to salinity stress in quinoa (Chenopodium quinoa Willd.) plants: Prospects for salinity tolerance and yield stability. Plant Physiology and Biochemistry 129:411420. doi:https://doi.org/10.1016/j.plaphy.2018.06.023 
Iraki NM, Singh N, Bressan RA, Carpita NC (1989) Cell Walls of Tobacco Cells and Changes in Composition Associated with Reduced Growth upon Adaptation to Water and Saline Stress. Plant Physiology 91 (1):48. doi:10.1104/pp.91.1.48

Isayenkov SV, Maathuis FJM (2019) Plant Salinity Stress: Many Unanswered Questions Remain. Frontiers in Plant Science 10 (80). doi:10.3389/fpls.2019.00080

Ivushkin K, Bartholomeus H, Bregt AK, Pulatov A, Bui EN, Wilford J (2018) Soil salinity assessment through satellite thermography for different irrigated and rainfed crops. International Journal of Applied Earth Observation and Geoinformation 68:230-237. doi:https://doi.org/10.1016/j.jag.2018.02.004

Ivushkin K, Bartholomeus H, Bregt AK, Pulatov A, Franceschini MHD, Kramer H, van Loo EN, Jaramillo Roman V, Finkers R (2019a) UAV based soil salinity assessment of cropland. Geoderma 338:502-512. doi:https://doi.org/10.1016/i.geoderma.2018.09.046

Ivushkin K, Bartholomeus H, Bregt AK, Pulatov A, Kempen B, de Sousa L (2019b) Global mapping of soil salinity change. Remote Sensing of Environment 231:111260. doi:https://doi.org/10.1016/j.rse.2019.111260

Jacobsen S-E (2003) The Worldwide Potential for Quinoa (Chenopodium quinoaWilld.). Food Reviews International 19 (1-2):167-177. doi:10.1081/FRI-120018883

Jacobsen SE, Christiansen JL (2016) Some Agronomic Strategies for Organic Quinoa (Chenopodium quinoa Willd.). Journal of Agronomy and Crop Science 202 (6):454-463. doi:10.1111/jac.12174

Jacobsen SE, Mujica A, Jensen CR (2003) The Resistance of Quinoa (Chenopodium quinoaWilld.) to Adverse Abiotic Factors. Food Reviews International 19 (1-2):99-109. doi:10.1081/fri-120018872

Jaikishun S, Li W, Yang Z, Song S (2019) Quinoa: In Perspective of Global Challenges. Agronomy 9 (4). doi:10.3390/agronomy9040176

Jaramillo Roman V, den Toom LA, Castro Gamiz C, van der Pijl N, Visser RGF, van Loo EN, van der Linden CG (2020) Differential responses to salt stress in ion dynamics, growth and seed yield of European quinoa varieties.

Environmental and Experimental Botany 177:104146. doi:https://doi.org/10.1016/j.envexpbot.2020.104146

Jarvis DE, Ho YS, Lightfoot DJ, Schmöckel SM, Li B, Borm TJA, Ohyanagi H, Mineta K, Michell CT, Saber N, Kharbatia NM, Rupper RR, Sharp AR, Dally N, Boughton BA, Woo YH, Gao G, Schijlen EGWM, Guo X, Momin AA, Negrão S, Al-Babili S, Gehring C, Roessner U, Jung C, Murphy K, Arold ST, Gojobori T, Linden CGvd, van Loo EN, Jellen EN, Maughan PJ, Tester M (2017) The genome of Chenopodium quinoa. Nature 542 (7641):307-312. doi:10.1038/nature21370

Jombart T (2008) Adegenet: A R package for the multivariate analysis of genetic markers. Bioinformatics 24 (11):1403-1405. doi:10.1093/bioinformatics/btn129

Julkowska MM, Testerink C (2015) Tuning plant signaling and growth to survive salt. Trends in Plant Science 20 (9):586-594. doi:https://doi.org/10.1016/j.tplants.2015.06.008

Jung NU, Giarola V, Chen P, Knox JP, Bartels D (2019) Craterostigma plantagineum cell wall composition is remodelled during desiccation and 
the glycine-rich protein CpGRP1 interacts with pectins through clustered arginines. The Plant Journal 100 (4):661-676. doi:10.1111/tpj.14479

Kadereit G, Borsch T, Weising K, Freitag H (2003) Phylogeny of Amaranthaceae and Chenopodiaceae and the Evolution of $\mathrm{C} 4$ Photosynthesis. International Journal of Plant Sciences 164 (6):959-986. doi:10.1086/378649

Kang SJ, Post WM, Nichols JA, Wang D, West TO, Bandaru V, Izaurralde RC (2013) Marginal lands: concept, assessment and management. Journal of Agricultural Science (Toronto) 5 (5):129-139

Karlberg L, Ben-Gal A, Jansson PE, Shani U (2006) Modelling transpiration and growth in salinity-stressed tomato under different climatic conditions. Ecological Modelling 190 (1-2):15-40. doi:10.1016/j.ecolmodel.2005.04.015

Katsoulas N, Baille A, Kittas C (2007) Leaf boundary layer conductance in ventilated greenhouses: An experimental approach. Agricultural and Forest Meteorology 144 (3):180-192. doi:https://doi.org/10.1016/j.agrformet.2007.03.003

Kiani-Pouya A, Roessner U, Jayasinghe NS, Lutz A, Rupasinghe T, Bazihizina N, Bohm J, Alharbi S, Hedrich R, Shabala S (2017) Epidermal bladder cells confer salinity stress tolerance in the halophyte quinoa and Atriplex species. Plant, Cell \& Environment 40 (9):1900-1915. doi:10.1111/pce.12995

Kimura K, Yasutake D, Yamanami A, Kitano M (2020) Spatial examination of leafboundary-layer conductance using artificial leaves for assessment of light airflow within a plant canopy under different controlled greenhouse conditions. Agricultural and Forest Meteorology 280:107773. doi:https://doi.org/10.1016/i.agrformet.2019.107773

Klaassen MT, Trindade LM (2020) RG-I galactan side-chains are involved in the regulation of the water-binding capacity of potato cell walls. Carbohydrate Polymers 227:115353. doi:https://doi.org/10.1016/j.carbpol.2019.115353

Koziol MJ (1991) Afrosimetric estimation of threshold saponin concentration for bitterness in quinoa (Chenopodium quinoa Willd). Journal of the Science of Food and Agriculture 54 (2):211-219. doi:10.1002/jsfa.2740540206

Kozioł MJ (1992) Chemical composition and nutritional evaluation of quinoa (Chenopodium quinoa Willd.). Journal of Food Composition and Analysis 5 (1):35-68. doi:https://doi.org/10.1016/0889-1575(92)90006-6

Kumar M, Campbell L, Turner S (2015) Secondary cell walls: biosynthesis and manipulation. Journal of Experimental Botany 67 (2):515-531. doi:10.1093/jxb/erv533

Lambers H, Poorter H (1992) Inherent Variation in Growth Rate Between Higher Plants: A Search for Physiological Causes and Ecological Consequences. Advances in Ecological Research, vol 23. doi:10.1016/s00652504(08)60148-8

Le Gall H, Philippe F, Domon J-M, Gillet F, Pelloux J, Rayon C (2015) Cell Wall Metabolism in Response to Abiotic Stress. Plants 4 (1):112-166

Leakey ADB, Ferguson JN, Pignon CP, Wu A, Jin Z, Hammer GL, Lobell DB (2019) Water Use Efficiency as a Constraint and Target for Improving the Resilience and Productivity of C3 and C4 Crops. Annual Review of Plant Biology 70 (1):781-808. doi:10.1146/annurev-arplant-042817-040305 
Lebaudy A, Véry A-A, Sentenac H (2007) K+ channel activity in plants: Genes, regulations and functions. FEBS Letters 581 (12):2357-2366. doi:https://doi.org/10.1016/j.febslet.2007.03.058

Leucci MR, Lenucci MS, Piro G, Dalessandro G (2008) Water stress and cell wall polysaccharides in the apical root zone of wheat cultivars varying in drought tolerance. Journal of Plant Physiology 165 (11):1168-1180. doi:https://doi.org/10.1016/j.jplph.2007.09.006

Li B, Tester M, Gilliham M (2017) Chloride on the Move. Trends in Plant Science 22 (3):236-248. doi:https://doi.org/10.1016/i.tplants.2016.12.004

Li H (2011) A statistical framework for SNP calling, mutation discovery, association mapping and population genetical parameter estimation from sequencing data. Bioinformatics 27 (21):2987-2993. doi:10.1093/bioinformatics/btr509

Li H, Durbin R (2009) Fast and accurate short read alignment with BurrowsWheeler transform. Bioinformatics 25 (14):1754-1760. doi:10.1093/bioinformatics/btp324

Li H, Handsaker B, Wysoker A, Fennell T, Ruan J, Homer N, Marth G, Abecasis G, Durbin R (2009) The Sequence Alignment/Map format and SAMtools. Bioinformatics 25 (16):2078-2079. doi:10.1093/bioinformatics/btp352

Lin HX, Zhu MZ, Yano M, Gao JP, Liang ZW, Su WA, Hu XH, Ren ZH, Chao DY (2004) QTLs for Na+ and K+ uptake of the shoots and roots controlling rice salt tolerance. Theor Appl Genet 108 (2):253-260. doi:10.1007/s00122003-1421-y

López-Marqués R, Nørrevang A, Ache P, Moog M, Visintainer D, Wendt T, Østerberg J, Dockter C, Jørgensen M, Salvador A, Hedrich R, Gao C, Jacobsen S-E, Shabala S, Palmgren M (2020) Prospects for the accelerated improvement of the resilient crop quinoa. Journal of experimental botany. doi:10.1093/jxb/eraa285

Luo M, Zhao Y, Zhang R, Xing J, Duan M, Li J, Wang N, Wang W, Zhang S, Chen Z, Zhang H, Shi Z, Song W, Zhao J (2017) Mapping of a major QTL for salt tolerance of mature field-grown maize plants based on SNP markers. BMC Plant Biology 17 (1):140. doi:10.1186/s12870-017-1090-7

Maathuis FJM, Ahmad I, Patishtan J (2014) Regulation of $\mathrm{Na}+$ fluxes in plants. Frontiers in Plant Science 5 (467). doi:10.3389/fpls.2014.00467

Maathuis FJM, Amtmann A (1999) K + Nutrition and $\mathrm{Na}+$ Toxicity: The Basis of Cellular K + / Na + Ratios. Annals of Botany 84 (2):123-133. doi:10.1006/anbo.1999.0912

Mastebroek HD, Limburg H, Gilles T, Marvin HJP (2000) Occurrence of sapogenins in leaves and seeds of quinoa (Chenopodium quinoa Willd). Journal of the Science of Food and Agriculture 80 (1):152-156. doi:10.1002/(SICI)1097-0010(20000101)80:1<152::AIDJSFA503>3.0.CO;2-P

Mastebroek HD, Van Loo EN, Dolstra O (2002) Combining ability for seed yield traits of Chenopodium quinoa breeding lines. Euphytica 125 (3):427-432. doi:10.1023/A:1016030129541

Matanguihan JB, Maughan PJ, Jellen EN, Kolano B (2015) Quinoa Cytogenetics, Molecular Genetics, and Diversity. In: Matanguihan KMaJ (ed) Quinoa: Improvement and Sustainable Production. doi:doi:10.1002/9781118628041.ch7 
Maughan PJ, Turner TB, Coleman CE, Elzinga DB, Jellen EN, Morales JA, Udall JA, Fairbanks DJ, Bonifacio A (2009) Characterization of Salt Overly Sensitive 1 (SOS1) gene homoeologs in quinoa (Chenopodium quinoa Willd.). Genome 52 (7):647-657. doi:10.1139/g09-041

McDermitt DK (1990) Sources of Error in the Estimation of Stomatal Conductance and Transpiration from Porometer Data. HortScience HortSci 25 (12):15381548. doi:10.21273/HORTSCI.25.12.1538

McElhinny E, Peralta E, Mazón N, Danial DL, Thiele G, Lindhout P (2007) Aspects of participatory plant breeding for quinoa in $\_$marginal areas of Ecuador. Euphytica 153 (3):373-384. doi:10.1007/s10681-006-9200-x

Medrano H, Escalona JM, Bota J, Gulías J, Flexas J (2002) Regulation of photosynthesis of C3 plants in response to progressive drought: stomatal conductance as a reference parameter. Annals of botany $89 \mathrm{Spec}$ No (7):895-905. doi:10.1093/aob/mcf079

Mishra A, Tanna B (2017) Halophytes: Potential resources for salt stress tolerance genes and promoters. Frontiers in Plant Science 8. doi:10.3389/fpls.2017.00829

Møller IS, Tester M (2007) Salinity tolerance of Arabidopsis: a good model for cereals? Trends in Plant Science 12 (12):534-540. doi:https://doi.org/10.1016/j.tplants.2007.09.009

Montes Osorio LR, Torres Salvador AF, Jongschaap REE, Azurdia Perez CA, Berduo Sandoval JE, Trindade LM, Visser RGF, van Loo EN (2014) High level of molecular and phenotypic biodiversity in Jatropha curcas from Central America compared to Africa, Asia and South America. BMC Plant Biology 14 (1). doi:10.1186/1471-2229-14-77

Moore JP, Farrant JM, Driouich A (2008a) A role for pectin-associated arabinans in maintaining the flexibility of the plant cell wall during water deficit stress. Plant Signaling \& Behavior 3 (2):102-104. doi:10.4161/psb.3.2.4959

Moore JP, Nguema-Ona EE, Vicré-Gibouin M, Sørensen I, Willats WGT, Driouich A, Farrant JM (2013) Arabinose-rich polymers as an evolutionary strategy to plasticize resurrection plant cell walls against desiccation. Planta 237 (3):739-754. doi:10.1007/s00425-012-1785-9

Moore JP, Vicré-Gibouin M, Farrant JM, Driouich A (2008b) Adaptations of higher plant cell walls to water loss: drought vs desiccation. Physiologia Plantarum 134 (2):237-245. doi:10.1111/j.1399-3054.2008.01134.x

Morton MJL, Awlia M, Al-Tamimi N, Saade S, Pailles Y, Negrão S, Tester M (2019) Salt stress under the scalpel - dissecting the genetics of salt tolerance. The Plant Journal 97 (1):148-163. doi:10.1111/tpj.14189

Moshelion M, Halperin O, Wallach R, Oren RAM, Way DA (2015) Role of aquaporins in determining transpiration and photosynthesis in waterstressed plants: crop water-use efficiency, growth and yield. Plant, Cell \& Environment 38 (9):1785-1793. doi:10.1111/pce.12410

Munns R (2002) Comparative physiology of salt and water stress. Plant, Cell and Environment 25 (2):239-250. doi:10.1046/j.0016-8025.2001.00808.x

Munns R (2005) Genes and salt tolerance: bringing them together. New Phytologist 167 (3):645-663. doi:10.1111/j.1469-8137.2005.01487.x

Munns R, Day DA, Fricke W, Watt M, Arsova B, Barkla BJ, Bose J, Byrt CS, Chen Z-H, Foster KJ, Gilliham M, Henderson SW, Jenkins CLD, Kronzucker HJ, 
Miklavcic SJ, Plett D, Roy SJ, Shabala S, Shelden MC, Soole KL, Taylor NL, Tester M, Wege S, Wegner LH, Tyerman SD (2020a) Energy costs of salt tolerance in crop plants. New Phytologist 225 (3):1072-1090. doi:10.1111/nph.15864

Munns R, Day DA, Fricke W, Watt M, Arsova B, Barkla BJ, Bose J, Byrt CS, Chen ZH, Foster KJ, Gilliham M, Henderson SW, Jenkins CLD, Kronzucker HJ, Miklavcic SJ, Plett D, Roy SJ, Shabala S, Shelden MC, Soole KL, Taylor NL, Tester M, Wege S, Wegner LH, Tyerman SD (2019) Energy costs of salt tolerance in crop plants. New Phytologist. doi:10.1111/nph.15864

Munns R, Gilliham M (2015) Salinity tolerance of crops - what is the cost? New Phytologist 208 (3):668-673. doi:10.1111/nph.13519

Munns R, James RA, Gilliham M, Flowers TJ, Colmer TD (2016) Tissue tolerance: an essential but elusive trait for salt-tolerant crops. Functional Plant Biology 43 (12):1103-1113. doi:https://doi.org/10.1071/FP16187

Munns R, James RA, Xu B, Athman A, Conn SJ, Jordans C, Byrt CS, Hare RA, Tyerman SD, Tester M, Plett D, Gilliham M (2012) Wheat grain yield on saline soils is improved by an ancestral $\mathrm{Na}^{+}$transporter gene. Nat Biotechnol 30 (4):360-364. doi:10.1038/nbt.2120

Munns R, Passioura JB, Colmer TD, Byrt CS (2020b) Osmotic adjustment and energy limitations to plant growth in saline soil. New Phytologist 225 (3):1091-1096. doi:10.1111/nph.15862

Munns R, Tester M (2008) Mechanisms of Salinity Tolerance. Annual Review of Plant Biology 59 (1):651-681. doi:10.1146/annurev.arplant.59.032607.092911

Murata N, Takahashi S, Nishiyama Y, Allakhverdiev SI (2007) Photoinhibition of photosystem II under environmental stress. Biochimica et Biophysica Acta - Bioenergetics 1767 (6):414-421. doi:10.1016/j.bbabio.2006.11.019

Murphy K, Matanguihan J (2015) Quinoa : improvement and sustainable production

Murphy KM, Bazile D, Kellogg J, Rahmanian M (2016) Development of a Worldwide Consortium on Evolutionary Participatory Breeding in Quinoa. Frontiers in plant science 7:608-608. doi:10.3389/fpls.2016.00608

Negin B, Moshelion M (2017) The advantages of functional phenotyping in pre-field screening for drought-tolerant crops. Functional Plant Biology 44 (1):107118. doi:10.1071/FP16156

Negrão S, Schmöckel SM, Tester M (2017) Evaluating physiological responses of plants to salinity stress. Annals of Botany 119 (1):1-11. doi:10.1093/aob/mcw191

Nguyen LV, Bertero D, Nguyen LV (2020) Genetic variation in root development responses to salt stresses of quinoa. Journal of Agronomy and Crop Science n/a (n/a). doi:10.1111/jac.12411

Norris IB (1982) Soil moisture and growth of contrasting varieties of Lolium, Dactylis and Festuca species. Grass and Forage Science 37 (4):273-283. doi:10.1111/j.1365-2494.1982.tb01607.x

Nowak V, Du J, Charrondière UR (2016) Assessment of the nutritional composition of quinoa (Chenopodium quinoa Willd.). Food Chemistry 193:47-54. doi:https://doi.org/10.1016/j.foodchem.2015.02.111

Nunez de Arco S (2015) Quinoa's Calling. In: Quinoa: Improvement and Sustainable Production. pp 211-226. doi:10.1002/9781118628041.ch12 
Okur B, Örçen N (2020) Chapter 12 - Soil salinization and climate change. In: Prasad MNV, Pietrzykowski M (eds) Climate Change and Soil Interactions. Elsevier, pp 331-350. doi:https://doi.org/10.1016/B978-0-12-8180327.00012-6

Orsini F, Accorsi M, Gianquinto G, Dinelli G, Antognoni F, Carrasco KBR, Martinez EA, Alnayef M, Marotti I, Bosi S, Biondi S (2011) Beyond the ionic and osmotic response to salinity in Chenopodium quinoa: Functional elements of successful halophytism. Functional Plant Biology 38 (10):818-831. doi:10.1071/fp11088

Ouyang W, Struik PC, Yin X, Yang J (2017) Stomatal conductance, mesophyll conductance, and transpiration efficiency in relation to leaf anatomy in rice and wheat genotypes under drought. Journal of Experimental Botany 68 (18):5191-5205. doi:10.1093/jxb/erx314

Peaucelle A, Braybrook S, Höfte H (2012) Cell wall mechanics and growth control in plants: the role of pectins revisited. Frontiers in plant science 3:121-121. doi:10.3389/fpls.2012.00121

Peleg Z, Apse MP, Blumwald E (2011) Chapter 12 - Engineering Salinity and Water-Stress Tolerance in Crop Plants: Getting Closer to the Field. In: Turkan I (ed) Advances in Botanical Research, vol 57. Academic Press, pp 405-443. doi:https://doi.org/10.1016/B978-0-12-387692-8.00012-6

Peralta E MN (2015) Quinoa in Ecuador. Chapter 5.3. In: D. Bazile DBCN (ed) State of the Art Report in Quinoa in the world in 2013. FAO, Rome, pp 388400

Peterson A, Murphy K (2015a) Tolerance of Lowland Quinoa Cultivars to Sodium Chloride and Sodium Sulfate Salinity. Crop Science 55 (1):331-338. doi:10.2135/cropsci2014.04.0271

Peterson AJ, Murphy K (2015b) Quinoa Cultivation for Temperate North America: Considerations and Areas for Investigation. In: Murphy K, Matanguihan J (eds) Quinoa: Improvement and Sustainable Production. pp 173-192. doi:10.1002/9781118628041.ch10

Petit J, Gulisano A, Dechesne A, Trindade LM (2019) Phenotypic Variation of Cell Wall Composition and Stem Morphology in Hemp (Cannabis sativa L.): Optimization of Methods. Frontiers in Plant Science 10 (959). doi:10.3389/fpls.2019.00959

Pieruschka R, Schurr U (2019) Plant Phenotyping: Past, Present, and Future. Plant Phenomics 2019:7507131. doi:10.34133/2019/7507131

Planella M, Lopez, M., Bruno, M. (2015) Domestication and prehistoric distribution. In: Bazile D, Bertero, D., Nieto, C. (ed) State of Art Report of Quinoa in the World 2013. FAO\&CIRAD, Rome,

Pulvento C, Riccardi M, Lavini A, lafelice G, Marconi E, d'Andria R (2012) Yield and Quality Characteristics of Quinoa Grown in Open Field Under Different Saline and Non-Saline Irrigation Regimes. Journal of Agronomy and Crop Science 198 (4):254-263. doi:10.1111/j.1439-037X.2012.00509.x

Qadir M, Quillérou E, Nangia V, Murtaza G, Singh M, Thomas RJ, Drechsel P, Noble AD (2014) Economics of salt-induced land degradation and restoration. Natural Resources Forum 38 (4):282-295. doi:10.1111/14778947.12054

R CT (2019) R: A language and environment for statistical computing. $R$ version 3.6.1 edn. R Foundation for Statistical Computing, Vienna, Austria 
Ralph PJ, Gademann R (2005) Rapid light curves: A powerful tool to assess photosynthetic activity. Aquatic Botany 82 (3):222-237. doi:10.1016/j.aquabot.2005.02.006

Rashidi B, Trindade LM (2018) Detailed biochemical and morphologic characteristics of the green microalga Neochloris oleoabundans cell wall. Algal Research 35:152-159. doi:https://doi.org/10.1016/j.algal.2018.08.033

Razzaghi F, Ahmadi SH, Adolf VI, Jensen CR, Jacobsen SE, Andersen MN (2011) Water Relations and Transpiration of Quinoa (Chenopodium quinoa Willd.) Under Salinity and Soil Drying. Journal of Agronomy and Crop Science 197 (5):348-360. doi:10.1111/j.1439-037X.2011.00473.x

Razzaghi F, Ahmadi SH, Jacobsen S-E, Jensen C, Andersen M (2012) Effects of Salinity and Soil-Drying on Radiation Use Efficiency, Water Productivity and Yield of Quinoa (Chenopodium quinoa Willd.). Journal of Agronomy and Crop Science 198:173-184. doi:10.1111/j.1439-037X.2011.00496.x

Razzaghi F, Bahadori-Ghasroldashti MR, Henriksen S, Sepaskhah AR, Jacobsen S-E (2020) Physiological characteristics and irrigation water productivity of quinoa (Chenopodium quinoa Willd.) in response to deficit irrigation imposed at different growing stages-A field study from Southern Iran. Journal of Agronomy and Crop Science 206 (3):390-404. doi:10.1111/jac.12392

Razzaghi F, Jacobsen S-E, Jensen CR, Andersen MN (2015) lonic and photosynthetic homeostasis in quinoa challenged by salinity and drought mechanisms of tolerance. Functional Plant Biology 42 (2):136-148. doi:https://doi.org/10.1071/FP14132

Reich PB, Wright IJ, Cavender-Bares J, Craine JM, Oleksyn J, Westoby M, Walters MB (2003) The Evolution of Plant Functional Variation: Traits, Spectra, and Strategies. International Journal of Plant Sciences 164 (S3):S143-S164. doi: $10.1086 / 374368$

Rengasamy P (2006) World salinization with emphasis on Australia. Journal of Experimental Botany 57 (5):1017-1023. doi:10.1093/jxb/erj108

Risi JC, Galwey NW (1989) The pattern of genetic diversity in the Andean grain crop quinoa (Chenopodium quinoa Willd). I. Associations between characteristics. Euphytica 41 (1):147-162. doi:10.1007/BF00022424

Rojas WP, M.; Alanoca, C.; Gomez Pando, L..; Leon Lobos, P.; Alercia, A.; Diulgheroff, S.; Padulosi, S.; Bazile, D. (2015) Quinoa genetic resources and ex situ convervation. In: D. Bazile HD, Bertero, and C. Nieto (ed) State of the Art Report on Quinoa Around the World in 2013. FAO\&CIRAD, Roma, pp 56-82

Roy SJ, Negrão S, Tester M (2014) Salt resistant crop plants. Current Opinion in Biotechnology 26:115-124. doi:https://doi.org/10.1016/j.copbio.2013.12.004

Roy SJ, Tucker EJ, Tester M (2011) Genetic analysis of abiotic stress tolerance in crops. Current Opinion in Plant Biology 14 (3):232-239. doi:10.1016/j.pbi.2011.03.002

Rubio F, Nieves-Cordones M, Horie T, Shabala S (2020) Doing 'business as usual' comes with a cost: evaluating energy cost of maintaining plant intracellular K+ homeostasis under saline conditions. New Phytologist 225 (3):10971104. doi: $10.1111 / \mathrm{nph} .15852$ 
Rui Y, Dinneny JR (2020) A wall with integrity: surveillance and maintenance of the plant cell wall under stress. New Phytologist 225 (4):1428-1439. doi:10.1111/nph.16166

Ruiz-Carrasco K, Antognoni F, Coulibaly AK, Lizardi S, Covarrubias A, Martínez EA, Molina-Montenegro MA, Biondi S, Zurita-Silva A (2011) Variation in salinity tolerance of four lowland genotypes of quinoa (Chenopodium quinoa Willd.) as assessed by growth, physiological traits, and sodium transporter gene expression. Plant Physiology and Biochemistry 49 (11):1333-1341. doi:https://doi.org/10.1016/i.plaphy.2011.08.005

Ruiz KB, Aloisi I, Del Duca S, Canelo V, Torrigiani P, Silva H, Biondi S (2016a) Salares versus coastal ecotypes of quinoa: Salinity responses in Chilean landraces from contrasting habitats. Plant Physiology and Biochemistry 101:1-13. doi:https://doi.org/10.1016/i.plaphy.2016.01.010

Ruiz KB, Biondi S, Martínez EA, Orsini F, Antognoni F, Jacobsen SE (2016b) Quinoa - a Model Crop for Understanding Salt-tolerance Mechanisms in Halophytes. Plant Biosystems - An International Journal Dealing with all Aspects of Plant Biology 150 (2):357-371. doi:10.1080/11263504.2015.1027317

Ruiz KB, Biondi S, Oses R, Acuña-Rodríguez IS, Antognoni F, Martinez-Mosqueira EA, Coulibaly A, Canahua-Murillo A, Pinto M, Zurita-Silva A, Bazile D, Jacobsen SE, Molina-Montenegro MA (2014) Quinoa biodiversity and sustainability for food security under climate change. A review. Agronomy for Sustainable Development 34 (2):349-359. doi:10.1007/s13593-0130195-0

Ruiz RA, Bertero HD (2008) Light interception and radiation use efficiency in temperate quinoa (Chenopodium quinoa Willd.) cultivars. European Journal of Agronomy 29 (2):144-152. doi:https://doi.org/10.1016/j.eja.2008.05.003

Sade N, Gebremedhin A, Moshelion M (2012) Risk-taking plants: anisohydric behavior as a stress-resistance trait. Plant signaling \& behavior 7 (7):767770. doi:10.4161/psb.20505

Sairam RK, Rao KV, Srivastava GC (2002) Differential response of wheat genotypes to long term salinity stress in relation to oxidative stress, antioxidant activity and osmolyte concentration. Plant Science 163

(5):1037-1046. doi:https://doi.org/10.1016/S0168-9452(02)00278-9

Salazar J, de Lourdes Torres M, Gutierrez B, Torres AF (2019) Molecular characterization of Ecuadorian quinoa (Chenopodium quinoa Willd.) diversity: implications for conservation and breeding. Euphytica 215 (3). doi:10.1007/s10681-019-2371-z

Sanders D (2020) The salinity challenge. New Phytologist 225 (3):1047-1048. doi:10.1111/nph.16357

Scaglione D, Pinosio S, Marroni F, Di Centa E, Fornasiero A, Magris G, Scalabrin S, Cattonaro F, Taylor G, Morgante M (2019) Single primer enrichment technology as a tool for massive genotyping: a benchmark on black poplar and maize. Annals of Botany 124 (4):543-551. doi:10.1093/aob/mcz054

Scheller HV, Ulvskov P (2010) Hemicelluloses. Annual Review of Plant Biology 61 (1):263-289. doi:10.1146/annurev-arplant-042809-112315

Schmöckel SM, Lightfoot DJ, Razali R, Tester M, Jarvis DE (2017) Identification of Putative Transmembrane Proteins Involved in Salinity Tolerance in 
Chenopodium quinoa by Integrating Physiological Data, RNAseq, and SNP Analyses. Frontiers in plant science 8:1023-1023.

doi:10.3389/fpls.2017.01023

Shabala L, Mackay A, Tian Y, Jacobsen S-E, Zhou D, Shabala S (2012) Oxidative stress protection and stomatal patterning as components of salinity tolerance mechanism in quinoa (Chenopodium quinoa). Physiologia Plantarum 146 (1):26-38. doi:10.1111/j.1399-3054.2012.01599.x

Shabala S (2013) Learning from halophytes: Physiological basis and strategies to improve abiotic stress tolerance in crops. Annals of Botany 112 (7):12091221. doi:10.1093/aob/mct205

Shabala S, Bose J, Hedrich R (2014) Salt bladders: do they matter? Trends in Plant Science 19 (11):687-691. doi:https://doi.org/10.1016/i.tplants.2014.09.001

Shabala S, Cuin TA (2008) Potassium transport and plant salt tolerance. Physiologia Plantarum 133 (4):651-669. doi:doi:10.1111/j.13993054.2007.01008.x

Shabala S, Hariadi Y, Jacobsen S-E (2013) Genotypic difference in salinity tolerance in quinoa is determined by differential control of xylem Na+ loading and stomatal density. Journal of Plant Physiology 170 (10):906914. doi:https://doi.org/10.1016/j.jplph.2013.01.014

Shabala S, Munns R (2017) Salinity stress: physiological constraints and adaptive mechanisms. In: Shabala S (ed) Plant stress physiology. CADI, UK, pp 2463

Sinclair TR (2018) Effective Water Use Required for Improving Crop Growth Rather Than Transpiration Efficiency. Frontiers in Plant Science 9 (1442). doi:10.3389/fpls.2018.01442

Sinclair TR, Muchow RC (1999) Radiation Use Efficiency. In: Sparks DL (ed) Advances in Agronomy, vol 65. Academic Press, pp 215-265. doi:https://doi.org/10.1016/S0065-2113(08)60914-1

Song J, Wang B (2014) Using euhalophytes to understand salt tolerance and to develop saline agriculture: Suaeda salsa as a promising model. Annals of Botany 115 (3):541-553. doi:10.1093/aob/mcu194

Spitters CJT, Schapendonk AHCM (1990) Evaluation of breeding strategies for drought tolerance in potato by means of crop growth simulation. Plant and Soil 123 (2):193-203. doi:10.1007/BF00011268

Srivastava P, Wu Q-S, Giri B (2019) Salinity: An Overview. In: Giri B, Varma A (eds) Microorganisms in Saline Environments: Strategies and Functions. Springer International Publishing, Cham, pp 3-18. doi:10.1007/978-3-03018975-4 1

Szczerba MW, Britto DT, Kronzucker HJ (2009) K+ transport in plants: Physiology and molecular biology. Journal of Plant Physiology 166 (5):447-466. doi:https://doi.org/10.1016/i.jplph.2008.12.009

Tanji KK (2002) Salinity in the Soil Environment. In: Läuchli A, Lüttge U (eds) Salinity: Environment - Plants - Molecules. Springer Netherlands, Dordrecht, pp 21-51. doi:10.1007/0-306-48155-3 2

Teakle NL, Tyerman SD (2010) Mechanisms of Cl- transport contributing to salt tolerance. Plant, Cell \& Environment 33 (4):566-589. doi:10.1111/j.13653040.2009.02060.x 
Tenhaken R (2015) Cell wall remodeling under abiotic stress. Frontiers in Plant Science 5 (771). doi:10.3389/fpls.2014.00771

Terashima I, Hanba YT, Tholen D, Niinemets Ü (2011) Leaf Functional Anatomy in Relation to Photosynthesis. Plant Physiology 155 (1):108. doi:10.1104/pp.110.165472

Tester M, Davenport R (2003) Na+ Tolerance and Na+ Transport in Higher Plants. Annals of Botany 91 (5):503-527. doi:10.1093/aob/mcg058

Thomson MJ, de Ocampo M, Egdane J, Rahman MA, Sajise AG, Adorada DL, Tumimbang-Raiz E, Blumwald E, Seraj ZI, Singh RK, Gregorio GB, Ismail AM (2010) Characterizing the Saltol Quantitative Trait Locus for Salinity Tolerance in Rice. Rice 3 (2):148-160. doi:10.1007/s12284-010-9053-8

Tschiersch H, Junker A, Meyer RC, Altmann T (2017) Establishment of integrated protocols for automated high throughput kinetic chlorophyll fluorescence analyses. Plant Methods 13 (1):54. doi:10.1186/s13007-017-0204-4

Turner NC (2018) Turgor maintenance by osmotic adjustment: 40 years of progress. Journal of Experimental Botany 69 (13):3223-3233. doi:10.1093/jxb/ery181

Tyerman SD, Munns R, Fricke W, Arsova B, Barkla BJ, Bose J, Bramley H, Byrt C, Chen Z, Colmer TD, Cuin T, Day DA, Foster K, Gilliham M, Henderson SW, Horie T, Jenkins CLD, Kaiser BN, Katsuhara M, Plett D, Miklavcic SJ, Roy SJ, Rubio F, Shabala S, Shelden M, Soole K, Taylor NL, Tester M, Watt M, Wege S, Wegner LH, Wen Z (2019) Energy costs of salinity tolerance in crop plants. New Phytologist 221 (1):25-29. doi:10.1111/nph.15555

USDA national nutrient database for dietary studies 2015-2016 (2020) FoodData Central Home Page. https://fdc.nal.usda.gov/index.html.

Uddin MN, Hanstein S, Leubner R, Schubert S (2013) Leaf Cell-Wall Components as Influenced in the First Phase of Salt Stress in Three Maize (Zea mays L.) Hybrids Differing in Salt Resistance. Journal of Agronomy and Crop Science 199 (6):405-415. doi:10.1111/jac.12031

Vadez V, Kholova J, Medina S, Kakkera A, Anderberg H (2014) Transpiration efficiency: new insights into an old story. J Exp Bot 65 (21):6141-6153. doi:10.1093/jxb/eru040

Van Loo EN (1992) Tillering, Leaf Expansion and Growth of Plants of Two Cultivars of Perennial Ryegrass Grown using Hydroponics at Two Water Potentials. Annals of Botany 70 (6):511-518. doi:10.1093/oxfordjournals.aob.a088511

Van Ooijen J (2009) MapQTL 6, Software for the mapping of quantitative trait loci in experimental populaitons. Kyazma B.V., Wageningen, Netherlands

van Zelm E, Zhang Y, Testerink C (2020) Salt Tolerance Mechanisms of Plants. Annual Review of Plant Biology 71 (1):403-433. doi:10.1146/annurevarplant-050718-100005

Vermerris W, Nicholson R (2006) Isolation and Identification of Phenolic Compounds. In: Vermerris W, Nicholson R (eds) Phenolic Compound Biochemistry. Springer Netherlands, Dordrecht, pp 151-196. doi:10.1007/978-1-4020-5164-7_4

Véry A-A, Nieves-Cordones M, Daly M, Khan I, Fizames C, Sentenac H (2014) Molecular biology of $\mathrm{K}+$ transport across the plant cell membrane: What do 
we learn from comparison between plant species? Journal of Plant Physiology 171 (9):748-769. doi:https://doi.org/10.1016/j.jplph.2014.01.011

Volkov V, Beilby MJ (2017) Editorial: Salinity Tolerance in Plants: Mechanisms and Regulation of Ion Transport. Frontiers in Plant Science 8 (1795). doi:10.3389/fpls.2017.01795

Voorrips RE (2002) MapChart: Software for the Graphical Presentation of Linkage Maps and QTLs. Journal of Heredity 93 (1):77-78.

doi:10.1093/jhered/93.1.77

Wang T, McFarlane HE, Persson S (2015) The impact of abiotic factors on cellulose synthesis. Journal of Experimental Botany 67 (2):543-552. doi:10.1093/jxb/erv488

Wani SH, Kumar V, Khare T, Guddimalli R, Parveda M, Solymosi K, Suprasanna P, Kavi Kishor PB (2020) Engineering salinity tolerance in plants: progress and prospects. Planta 251 (4):76. doi:10.1007/s00425-020-03366-6

Wicke B, Smeets E, Dornburg V, Vashev B, Gaiser T, Turkenburg W, Faaij A (2011) The global technical and economic potential of bioenergy from saltaffected soils. Energy \& Environmental Science 4 (8):2669-2681. doi:10.1039/C1EE01029H

Wieme RA, Carpenter-Boggs LA, Crowder DW, Murphy KM, Reganold JP (2020a) Agronomic and economic performance of organic forage, quinoa, and grain crop rotations in the Palouse region of the Pacific Northwest, USA.

Agricultural Systems 177:102709. doi:https://doi.org/10.1016/j.agsy.2019.102709

Wieme RA, Reganold JP, Crowder DW, Murphy KM, Carpenter-Boggs LA (2020b) Productivity and soil quality of organic forage, quinoa, and grain cropping systems in the dryland Pacific Northwest, USA. Agriculture, Ecosystems \& Environment 293:106838. doi:https://doi.org/10.1016/j.agee.2020.106838

Willats WGT, McCartney L, Mackie W, Knox JP (2001) Pectin: cell biology and prospects for functional analysis. Plant Molecular Biology 47 (1):9-27. doi:10.1023/A:1010662911148

Wolf S, Hématy K, Höfte H (2012) Growth Control and Cell Wall Signaling in Plants. Annual Review of Plant Biology 63 (1):381-407. doi:10.1146/annurev-arplant-042811-105449

Wu H, Zhang X, Giraldo JP, Shabala S (2018) It is not all about sodium: revealing tissue specificity and signalling roles of potassium in plant responses to salt stress. Plant and Soil 431 (1-2). doi:10.1007/s11104-018-3770-y

Wungrampha S, Joshi R, Singla-Pareek SL, Pareek A (2018) Photosynthesis and salinity: are these mutually exclusive? Photosynthetica 56 (1):366-381. doi:10.1007/s11099-017-0763-7

Yamaguchi T, Blumwald E (2005) Developing salt-tolerant crop plants: challenges and opportunities. Trends in Plant Science 10 (12):615-620. doi:https://doi.org/10.1016/i.tplants.2005.10.002

Yasui Y, Hirakawa H, Oikawa T, Toyoshima M, Matsuzaki C, Ueno M, Mizuno N, Nagatoshi Y, Imamura T, Miyago M, Tanaka K, Mise K, Tanaka T, Mizukoshi H, Mori M, Fujita Y (2016) Draft genome sequence of an inbred line of Chenopodium quinoa, an allotetraploid crop with great environmental adaptability and outstanding nutritional properties. DNA Research 23 (6):535-546. doi:10.1093/dnares/dsw037 
Yoo CY, Pence HE, Hasegawa PM, Mickelbart MV (2009) Regulation of Transpiration to Improve Crop Water Use. Critical Reviews in Plant Sciences 28 (6):410-431. doi:10.1080/07352680903173175

Yuan F, Leng B, Wang B (2016) Progress in Studying Salt Secretion from the Salt Glands in Recretohalophytes: How Do Plants Secrete Salt? Frontiers in Plant Science 7 (977). doi:10.3389/fpls.2016.00977

Zebrowski C Los suelos con cangahua en el Ecuador. In: III Simposio Internacional sobre suelos volcanicos endurecidos, Quito, 1996.

Zhang H, Yin W, Xia X (2010) Shaker-like potassium channels in Populus, regulated by the CBL-CIPK signal transduction pathway, increase tolerance to low-K+ stress. Plant Cell Reports 29 (9):1007-1012. doi:10.1007/s00299-010-0886-9

Zhao C, Zayed O, Zeng F, Liu C, Zhang L, Zhu P, Hsu C-C, Tuncil YE, Tao WA, Carpita NC, Zhu J-K (2019) Arabinose biosynthesis is critical for salt stress tolerance in Arabidopsis. New Phytologist 224 (1):274-290. doi:10.1111/nph.15867

Zhou G, Johnson P, Ryan PR, Delhaize E, Zhou M (2012) Quantitative trait loci for salinity tolerance in barley (Hordeum vulgare L.). Molecular Breeding 29 (2):427-436. doi:10.1007/s11032-011-9559-9

Zörb C, Geilfus CM, Dietz KJ (2019) Salinity and crop yield. Plant Biology 21 (S1):31-38. doi:10.1111/plb.12884

Zou C, Chen A, Xiao L, Muller HM, Ache P, Haberer G, Zhang M, Jia W, Deng P, Huang R, Lang D, Li F, Zhan D, Wu X, Zhang H, Bohm J, Liu R, Shabala S, Hedrich R, Zhu J-K, Zhang H (2017) A high-quality genome assembly of quinoa provides insights into the molecular basis of salt bladder-based salinity tolerance and the exceptional nutritional value. Cell Research 27 (11):1327-1340. doi:10.1038/cr.2017.124

Zurita-Silva A, Fuentes F, Zamora P, Jacobsen SE, Schwember AR (2014) Breeding quinoa (Chenopodium quinoa Willd.): Potential and perspectives. Molecular Breeding 34 (1):13-30. doi:10.1007/s11032-014-0023-5 


\section{Summary}

Soil salinization is a serious threat to agriculture, jeopardizing global food security and causing huge economic losses every year. Plants face formidable challenges when growing under saline conditions. The high concentration of ions in the root environment hampers water uptake, yet the accumulation of ions in plant tissues induces cytotoxicity. Unfortunately, soil salinization is likely to increase in the context of climate change, which lead to sea level rising and shortage of fresh-water sources. Hence, crop production might rely on irrigation with brackish/ saline water. In addition, the increasing global food demand calls for cultivation in less favourable soils including saline deltas around the world. Improvement of salt tolerance of existing crops might not be sufficient under increasing salinization, so the exploration of new, more salt tolerant crops appears to be essential to ensure food supply. Chenopodium quinoa is -at least outside S. America- a relatively new crop that has gained ground due to its resilience to withstand abiotic stress, especially soil salinity. Quinoa can potentially contribute to ensure food security and it has a healthy balance of proteins with all the essential amino acids, carbohydrates (starch), fibre and seed oil. In this thesis novel insights on the mechanisms and processes that underlie the remarkable salt tolerance of quinoa are provided and traits that may be used to improve crop salt tolerance are described.

In Chapter 2 we evaluate responses of quinoa varieties to long-term salinity (0$400 \mathrm{mM} \mathrm{NaCl}$ ) with respect to growth and ion homeostasis. We found that salinity reduced the seed yield of the varieties (grown as spaced plants in the greenhouse) by on average $29 \%$ at $100 \mathrm{mM} \mathrm{NaCl}$ and up to $88 \%$ at $400 \mathrm{mM} \mathrm{NaCl}$. We observed that quinoa varieties utilise salt exclusion strategies to produce relatively high yields under mild salinity or short-term stress, while tissue tolerance mechanisms enable the plants to survive and even reproduce under severe and prolonged salinity stress. In addition, $\left[\mathrm{K}^{+}\right]$in young leaves increased under salinity, especially at high salt concentrations. Likely, $\mathrm{K}^{+}$plays a role in leaf osmotic adjustment, and also protects the cells from metabolic failure due to a low $\mathrm{K}^{+} / \mathrm{Na}^{+}$when $\mathrm{Na}^{+}$reaches high concentrations in the leaves. We also examined the potential contribution of epidermal bladder cells (EBCs) to the ion homeostasis of quinoa. EBCs accumulated $5.4 \%$ and $6.5 \%$ of the total $\mathrm{Na}^{+}$and $\mathrm{Cl}^{-}$in the leaves, which indicates that the storage of salt in EBCs is not likely to contribute significantly to reduce levels of ions in the leaves. 
In Chapter 3 we focused on changes in leaf and stem cell wall composition under a salt stress of $400 \mathrm{mM} \mathrm{NaCl}$. We found that salinity alters the cell wall composition by decreasing the lignin and cellulose content and increasing pectin content. The highest variation in pectin monosaccharides was found for arabinose that increased by $160 \%$ in stems and $60 \%$ in leaves. The mineral composition of the cell wall was also affected by salinity: $\mathrm{Ca}^{2+}$ decreased by $30 \%$ and $65 \%$ while $\mathrm{Na}^{+}$increased by $140 \%$ and $70 \%$ in stem and leaf cell walls, respectively. We suggest that these changes increase the flexibility and hydration of the cell wall, which might help the cells to cope with changes in osmotic potential and turgor imposed by salinity.

In Chapter 4 we investigated the salinity-induced changes in water relations of two varieties with different responses to salinity: Pasto and selRiobamba. To this end, we implemented the Plantarray 3.0 phenotyping platform ${ }^{\circledR}$ to monitor changes in growth and transpiration with a high temporal resolution. Salinity reduced the cumulative transpiration of both varieties by $60 \%$ at $200 \mathrm{mM} \mathrm{NaCl}$ and by $75 \%$ and $82 \%$ at $300 \mathrm{mM} \mathrm{NaCl}$ for selRiobamba and Pasto, respectively. Stomatal conductance was reduced by salinity, but at $200 \mathrm{mM} \mathrm{NaCl}$ Pasto showed a lower reduction (15\%) than selRiobamba (35\%), along with decreased specific leaf area. Both varieties had increased water use efficiency under salt stress. We propose that contrasting water management strategies contribute to the differences in salt tolerance between Pasto and selRiobamba. Pasto adopted a "conservative-growth" strategy, saving water at the expense of growth, while selRiobamba used an "acquisitive-growth" strategy, maximising growth in spite of the stress.

In Chapters 2, 3 and 4 responses of quinoa to salinity were explored using single plants as experimental units. In Chapter 5 we switched to field-like conditions, using crop-like plant densities ( $\sim 50$ plants. $\left.\mathrm{m}^{-2}\right)$ and a salt treatment of $250 \mathrm{mM} \mathrm{NaCl}$. We evaluated the agronomic performance (seed yield, thousand seed weight, harvest index) and physiological responses to salinity of six commercial varieties. In addition, we used bi-parental crosses from varieties with contrasting salt tolerance responses to discover genetic factors of traits contributing to salt tolerance of quinoa. The salt tolerance of the varieties based on seed yield ranged from 68 to $92 \%$. The average salt tolerance was $67 \%$ for the mapping population Atlas $\times$ Red Carina and $75 \%$ for the Pasto $x$ Red Carina population. We found QTLs for all the analysed traits and identified putative alleles donated by Pasto associated to lower $\mathrm{Na}^{+}$and $\mathrm{Cl}^{-}$contents, higher $\mathrm{K}^{+}$retention and lower SLA that likely contribute to salt tolerance in the Pasto $x$ Red Carina population. 
Natural variation in agronomical and salt stress tolerance traits within species is a pre- requisite for crop improvement through breeding. In Chapter $\mathbf{6}$ we evaluated the genetic diversity of 22 genotypes selected as good representatives of the genetic diversity of quinoa in Ecuador. These accessions were grown under a 12-h light photoperiod and field-crop density under control conditions and a salt treatment of $250 \mathrm{mM} \mathrm{NaCl}$. All the genotypes proved to be highly salt tolerant with an average Salt Tolerance Index based on seed yield of $78 \%$. This collection was highly diverse for agronomical traits: the seed yield under control conditions ranged from $890-1145 \mathrm{~g} / \mathrm{m}^{2}$ and the thousand seed weight ranged from $2.4-4.4 \mathrm{~g} / 1000$ seeds. This collection was diverse for salt tolerance traits as well. $\mathrm{Na}^{+}$exclusion was the preferred mechanism under this, for quinoa, "mild" salinity stress, but some genotypes accumulated high concentrations of $\mathrm{Na}^{+}$in the leaves. The results reported in this Chapter demonstrate that the Inter-Andean valley ecotype from the Ecuadorian highlands is highly diverse and constitutes an interesting genetic resource for salt tolerance and other breeding purposes.

In Chapter $7 \mathrm{I}$ integrate the insights presented in this thesis on how quinoa responds to salt stress and analyse the potential use of this species to identify traits that might increase the salt tolerance of current crops. I propose an ideotype for salt tolerance characterized by a high rate of $\mathrm{Na}^{+}$and $\mathrm{Cl}^{-}$exclusion from leaves, high $\mathrm{K}^{+}$ retention (when $\mathrm{Na}^{+}$in the shoot rises), responsive control of stomata opening, among other plastic traits associated with an "acquisitive" growth under moderate stress and "conservative" growth under severe salt stress. Finally, I reflect on how salt tolerance in particular, and resilience to abiotic stresses in general, are quinoa's strongest asset to strengthen as an emerging staple crop. 



\section{Resumen}

La salinización de los suelos es una seria amenaza para la agricultura que compromete la seguridad alimentaria y causa inmensas pérdidas económicas cada año. Las plantas enfrentan formidables desafíos al crecer en suelos salinos. La gran concentración de iones alrededor de las raíces dificulta la absorción de agua y, adicionalmente, la acumulación de iones en los tejidos causa citotoxicidad. Desafortunadamente, la salinización de suelos incrementará en los próximos años, muy vinculada al cambio climático que generará un incremento en los niveles del mar e, indirectamente, promoverá la escasez de agua dulce. Por esta razón, la producción agrícola dependerá cada vez más de irrigación con agua salobre/ salina. Adicionalmente, la creciente demanda mundial de alimentos constituye una presión permanente para cultivar en suelos marginales, incluyendo los deltas salinos alrededor del mundo. Mejorar la tolerancia a salinidad de los cultivos existentes probablemente no sea suficiente frente a la creciente salinización de los suelos, por lo que la exploración de nuevos cultivos más tolerantes será esencial para garantizar el suministro de alimentos. Chenopodium quinoa es, al menos fuera de América del Sur, un cultivo relativamente nuevo que ha ganado terreno debido a su alta resistencia a estrés abiótico, especialmente salinidad. La quinua puede potencialmente contribuir a la seguridad alimentaria por tener un balance adecuado de proteínas con todos los aminoácidos esenciales, carbohidratos (almidón), fibra y aceites esenciales. Esta tesis proporciona nuevos conocimientos sobre los mecanismos responsables de la gran tolerancia a salinidad de la quinua, y potenciales indicadores genéticos que podrían utilizarse para mejorar la tolerancia a salinidad de otros cultivos.

En el Capítulo 2 evaluamos el crecimiento y balance de iones de algunas variedades de quinua expuestas a diferentes niveles de sal $(0-400 \mathrm{mM} \mathrm{NaCl})$ a largo plazo. El estrés salino redujo el rendimiento de las variedades (cultivadas en macetas en el invernadero) en un $29 \%$ en promedio bajo una concentración de 100 $\mathrm{mM} \mathrm{NaCl}$ y $88 \%$ bajo una concentración de $400 \mathrm{mM} \mathrm{NaCl}$. Observamos que las variedades utilizan mecanismos de exclusión de iones para mantener un alto rendimiento bajo estrés moderado o a corto plazo, mientras que mecanismos asociados con tolerancia de iones en los tejidos son empleados para sobrevivir, e incluso producir semillas, bajo estrés severo o a largo plazo. Adicionalmente la concentración de $\mathrm{K}^{+}$en hojas jóvenes aumentó en plantas cultivadas bajo estrés salino, especialmente en los niveles más elevados de sal. Probablemente el $\mathrm{K}^{+}$ 
cumple una función en el ajuste osmótico de las hojas, al mismo tiempo que protege las células de la citotoxicidad ocasionada por un desproporcionado balance entre $\mathrm{Na}^{+}$y $\mathrm{K}^{+}$. Finalmente, evaluamos la contribución de las vesículas epiteliales (EBCs por sus siglas en inglés) en el equilibrio iónico de las plantas. Las EBCs acumularon un $5.4 \%$ y $6.5 \%$ del total de $\mathrm{Na}^{+}$y $\mathrm{Cl}^{-}$presente en las hojas, lo que indica que la acumulación de sal en EBCs no contribuye significativamente a reducir los niveles de iones contenidos en las hojas.

En el Capítulo 3 nuestro objetivo fue analizar cambios en la composición de las paredes celulares de hojas y tallos bajo estrés salino (400 mM NaCl). Descubrimos que la salinidad altera la composición de la pared celular al disminuir el contenido de lignina y celulosa y aumentar el contenido de pectina. La mayor variación en los monosacáridos que conforman pectinas se encontró en arabinosa, que incrementó en un $160 \%$ en los tallos y en un 60 \% en las hojas. La composición mineral de la pared celular también se vio afectada por la salinidad: el $\mathrm{Ca}^{2+}$ disminuyó en un $30 \%$ y un $65 \%$ mientras que el $\mathrm{Na}^{+}$aumentó en un $140 \%$ y un 70 $\%$ en las paredes celulares del tallo y las hojas, respectivamente. Sugerimos que estos cambios aumentan la flexibilidad y la hidratación de la pared celular, lo que podría ayudar a las células a adaptarse a los cambios en el potencial osmótico y la turgencia impuestos por la salinidad.

En el Capítulo 4 investigamos los cambios inducidos por el estrés salino en el balance hídrico de dos variedades de quinua (Pasto y selRiobamba) expuestas a diferentes niveles de salinidad. Con este propósito, implementamos la plataforma de fenotipado Plantarray $3.0 \circledR$ para monitorear cambios en el crecimiento y la transpiración de las plantas en tiempo real. La salinidad redujo la transpiración acumulada de ambas variedades en un $60 \%$ bajo el tratamiento de $200 \mathrm{mM} \mathrm{NaCl}$ y en un $75 \%$ y $82 \%$ bajo $300 \mathrm{mM} \mathrm{NaCl}$ en selRiobamba y Pasto, respectivamente. La conductividad estomática también fue reducida por la salinidad, pero bajo el tratamiento de $200 \mathrm{mM} \mathrm{NaCl}$, la disminución fue menor en Pasto (15 \%) que en selRiobamba (35\%), debido a sus diferencias en la reducción del área foliar específica. En ambas variedades el estrés salino causó un incremento en la eficiencia en el uso de agua. Proponemos que las estrategias empleadas en el uso de agua contribuyen a las diferencias en tolerancia a salinidad entre ambas variedades. Pasto utiliza una "estrategia conservadora", ahorrando agua a expensas de un crecimiento desacelerado, mientras que selRiobamba utiliza una "estrategia adquisitiva", maximizando su crecimiento a pesar del estrés salino. 
En los Capítulos 2, 3 y 4 exploramos las respuestas frente a la salinidad en plantas de quinua cultivadas en macetas. En el Capítulo 5, nos enfocamos en condiciones de campo, semejando las condiciones óptimas de cultivo ( $~ 50$ plantas/ $\mathrm{m}^{2}$ ) y utilizando un nivel de salinidad moderado para la quinua (250 mM $\mathrm{NaCl}$ ). Evaluamos el desempeño agronómico (rendimiento de semillas, peso de mil semillas, índice de cosecha) y respuestas fisiológicas frente al estrés salino de seis variedades comerciales. Adicionalmente, evaluamos dos cruces biparentales entre variedades de quinua con respuestas contrastantes frente a salinidad, con el fin de detectar indicadores o rasgos genéticos asociados con la tolerancia a salinidad de esta especie. La tolerancia a la sal de las variedades basada en el rendimiento de semillas osciló entre el 68 y el $92 \%$. La tolerancia promedio a la sal fue del $67 \%$ para la población de mapeo Atlas x Red Carina y del 75 \% para la población Pasto $x$ Red Carina. Encontramos QTLs para todos los rasgos analizados e identificamos alelos putativos donados por Pasto asociados a menores contenidos de $\mathrm{Na}^{+} \mathrm{y} \mathrm{Cl}^{-}$, mayor retención de $\mathrm{K}^{+}$y menor área foliar específica, que probablemente contribuyen a la mayor tolerancia a la sal observada en la población Pasto x Red Carina.

La variación natural inter-específica es un requisito esencial en los programas de mejoramiento de un cultivo. Por esta razón, en el Capítulo 6 evaluamos la diversidad genética de 22 genotipos que representan la diversidad de quinua cultivada en la actualidad en Ecuador. Estas accesiones fueron cultivadas bajo un fotoperiodo de 12 horas de luz, semejando densidad de producción en campo, bajo control y un tratamiento de salinidad de $250 \mathrm{mM} \mathrm{NaCl}$. Todos los genotipos mostraron ser tolerantes a salinidad y el índice de estrés salino promedio fue de 78 \%. La colección mostró ser altamente diversa para diferentes propiedades agronómicas: el rendimiento de semilla bajo control varió de $890-1145 \mathrm{~g} / \mathrm{m}^{2}$ y el peso de mil semillas varió de 2.4 a $4.4 \mathrm{~g}$. Las accesiones también variaron en su respuesta al estrés salino. El principal mecanismo de tolerancia frente a estas condiciones de estrés "moderado" para quinua fue la exclusión de iones en las hojas; sin embargo, en algunas accesiones se pudo observar alta concentración de sales. Los resultados reportados en este capítulo demuestras que el ecotipo del valle interandino de la Sierra ecuatoriana es altamente diverso y constituye un interesante recurso genético para tolerancia a estrés salino y otros rasgos de interés.

En el Capítulo 7 integro las ideas presentadas a través de la tesis sobre cómo la quinua responde a estrés salino y analizo el potencial de esta empresa como fuente de mecanismos novedosos de tolerancia que podrían ser 
potencialmente trasladados a otros cultivos. Propongo un ideotipo de quinua tolerante a sal caracterizado por alto grado de exclusión de $\mathrm{Na}^{+} \mathrm{y} \mathrm{Cl}^{-}$de las hojas, alta retención de $\mathrm{K}^{+}$(sobre todo cuando la concentración de $\mathrm{Na}^{+}$incrementa), rápido control en la apertura de estomas, entre otros rasgos que caracterizan un metabolismo "adquisitivo" bajo estrés moderado y "conservativo" bajo estrés severo. Finalmente, reflexiono sobre cómo la tolerancia a salinidad en particular, y la resiliencia frente a estrés abiótico en general, son el activo más fuerte de la quinua para consolidarse como cultivo básico emergente 


\section{Acknowledgements}

Writing the acknowledgements of this thesis is a unique opportunity to recall my memories of the last five years and pay a small tribute to the incredible people who have been next to me throughout this amazing journey. I will try to do it chronologically.

Before all this started, I had my luggage ready to travel to Argentina and start a PhD in INTA under the supervision of Dr. Esteban Hopp. Undoubtedly, I am here now thanks to Andres Torres, who with all his tenacity encouraged me to apply to Wageningen UR, managed to get me an interview, and convinced Plant Breeding to trust this stranger who could only provide the enthusiasm to work with quinoa. It feels like yesterday that I received my invitation letter signed by prof. Richard Visser; which was also the same day I communicated my decision to my almost supervisor in Argentina. I also clearly remember his reply: "Viviana, I would do the same if I were you; go, dream high, and make us all proud." I will treasure those words forever.

Andres, we have come a long way from those days; thanks for being part of the decision that has defined the rest of my life! And for being such an important part of it.

From the day I received my acceptance letter and on, my eyes were stuck to my computer screen following every single e-mail that came from the other side of the world. Some names started to take shape in my mind and my heart. Prof. Richard Visser, thanks for your trust and for giving me the opportunity to join your group. You project an image of authority and trust that makes any decision simpler. I admire your exemplary leadership and feel extremely proud of being your $150^{\text {th }} \mathrm{PhD}$ candidate. Dr. Gerard van der Linden, I wish my vocabulary was rich enough to describe my admiration and gratitude to you. I can describe you as a wise and calm mentor, brilliant scientist, and thoughtful supervisor. I know things are changing in your life, but I am sure you have the wisdom to adapt and keep inspiring the people that have the privilege to get to know you. Apart from science, you have followed my steps like a father in the Netherlands, and I can't be more grateful for that. I hope we have built a sincere friendship; my esteem will always stand alive. Dr. Robert van Loo, you taught me perhaps the correct use of an e-mail. You repeated constantly: "If you need me, call me." And I can confirm that you were always there whenever I called, and that is maybe the best way I can describe you. You are always present, with your brilliant mind, your precision, your knowledge, and your kindness. I knew every day of this journey that you had my back and I can't be happier that our time 
and work with quinoa hasn't reach an end. I appreciate and treasure everything you have taught me.

To my three supervisors: I am not ready to leave; so, I hope you don't mind if I stick around for a cup of tea, some advice, or to find any creative opportunity to keep what we built alive. I count myself lucky for having had your mentorship.

I was lucky enough to never need an external supervisor's input. However, I had the privilege to count on a "side advisor," prof. Luisa Trindade. You always welcomed me in your office and your group. Thank you so much for your guidance and kindness. You are truly an inspiration to me! I also want to acknowledge my mentor from my hometown, Dr. Maria de Lourdes Torres. I would not be where I am today without your guidance. Thanks to Dr. René Smulders for your kindness and encouragement throughout these years.

I had the privilege to be welcomed to the Netherlands by my most generous friend, Sanne Mirck. Thanks for introducing me to your favourite childhood sweets and memories, for teaching me that there is no better place in this country than Zwolle, and that a broken heart can be fixed by riding a bike. You, Andres, Salito, and Maite are certainly family!

It couldn't be a coincidence that I arrived to an office with a group of people crazy enough to squeeze two gigantic banana plants, one bamboo tree, one dead miscanthus, and all kinds of orchids and bromelias next to the window. Former officemates, forever friends-thanks for the magic!

This book summarizes six quinoa growing seasons in the greenhouse and three in the field. My name might be on the cover, but a whole team is behind each page. To my quinoa team: it was a pleasure to work and learn with you. Arno den Tom, you tested the salt tolerance of Dutch quinoa varieties even before I came to Wageningen; Carlos Castro-Gamiz, you actually introduced me to the lab; Niels van der Pijl, I can't imagine a better companion for the nocturnal measurements of quinoa transpiration; Leah Nandudu, your hard work and positive energy always inspired me; Edoardo Vergnano and María Sánchez, thanks for your patient skills in the biochemical lab; Collins Agho and Olewaseun Ankilade, you have the strength and the mindset needed to succeed; Roberto Ramírez, you were undoubtedly my right hand in the field and I am sure we built a very strong friendship; Michael Cahalane, thanks for your smart ideas and efficiency; Yuxiao Zeng and Samet Saglat, thanks for all the exhausting hours in the field, Hugo Rijken, thanks for your bright mind and skills behind the computer; Mathijs Peters, I was not your supervisor, but you were, and are, a key member of the quinoa team. 
My second quinoa team was the amazing Unifarm crew. Andre Maassen, thanks for always finding a last-minute spot for me in the greenhouses. Geurt Versteeg, I was so confident knowing that my plants were happier and healthier in your hands than in mine. Sean Geurts and Rohan van Genderen, thanks for your time, your willingness to always give me a hand, and thanks for your friendship. Herman Meurs, Wim van der Slikke, and Gerrit Huisman, thanks for always being around and taking care of me. My days in Unifarm were simply beautiful. I can't mention every single team member in Unifarm, but your work and kindness keep all the projects and the dreams behind them alive. Your effort is greatly appreciated.

With the support from my supervisors, in 2016 I had the opportunity to go back to Ecuador to collect grain samples. Thanks to all the friends and family members who joined the various field trips. And thanks to all the communities and quinoa growers that provided me with samples. I have kept a list of all the growers that participated in this collection, I can't name them here, but I definitely collected more than grain on those trips.

Along the way I was blessed to make wonderful friends, most of them sharing the same dreams and facing the same difficulties. To my beloved BBE friends: Tim, Xuan, Jordi, Kasper, Francesco, and Agata, thanks for making this journey fun! You made the office messy and my heart warm! We might end up in different places, but I hope we can always come back to our beautiful memories, the laughs, the tears, and the complicity of PhD life. You have a special place in my heart! Agata and Francesco, thanks for being my paranymphs. I am not sure if we will be together on the stage or behind a screen, but I am sure that having you close will make me happy! To my other PhD mates: Mas, Marine, Charlotte, thanks for being the best companions for trips, birthdays, and adventures-one day we will take that train to Iran! Peter, thanks for your brightness; Anne, for your transparency; Mathilde, for your generosity; Ramon, for your loyalty; Kim, for your sweetness; Miguel for your happiness, and Marce and Rafa, for your enthusiasm! Caro and Diego, thanks for helping me disconnect from $\mathrm{PhD}$ talk when it was needed.

PBR is a very big family! Thanks to all the members who supported me all these years. A special thanks to Clemens van de Wiel, Elly Janssen, and Hanneke van der Schoot, the Abiotic Stress Group. Thanks for the help, the advice, and your permanent support! Marian Oortwijn and Johan Bucher, it is a privilege to consider you my friends. Nicole Trefflich and Danielle van der Wee, thanks for your invaluable help. In addition to your help, thanks for your enthusiasm that holds the PBR team together! Dianka Dees and Jos Molthoff, the COVID situation didn't 
allow me to share much time with you in the office, but you were part of very crucial times of this journey! Linda Kodde, Elma Salentijn, Christel Denneboom, Heleen Furrer, Marcel Visser, Gert van Arkel, and Isolde Bertram thanks for your kindness and contributions to my project and my daily activities all these years! A special thanks to Annemarie Dechesne for showing me the way in the Biochemical Lab; thanks for your knowledge, experience and your trust.

Marlene Minderhoud, thanks for the difficult (and in progress) task of teaching me Dutch!

To my small precious Iranian community: Sannaz, Delaram, Mohammad Reza, Farzad, thanks for the permanent support, for giving me the opportunity to feel the kindness of the Persian culture, and for sharing common dreams and failures that come along with the challenge of settling down in a new home. Mehdi and Libby (we can consider ourselves part of this luxury club!), thanks for the memories that we have just started to build, the familiarity, and a promising lifetime friendship!

Apologies for switching to Spanish to acknowledge the group of people who have witnessed my ups and downs throughout these years and beyond.

Tengo el enorme privilegio de tener una familia que ha sido mi soporte y $\mathrm{mi}$ inspiración en cada paso de mi vida. El día de mi despedida, me preguntaste Mami: ¿serías más feliz si te quedaras? La respuesta estaba clara y gracias por, a pesar de tu tristeza, poner perspectiva a cada decisión en mi vida. Gracias por enseñarme a soñar y por ser ejemplo de una mujer luchadora y sabia. Papi, vivir lejos me ha demostrado que tenemos más en común de lo que un día pensé. Hoy solo puedo admirar la pasión e intensidad con la que has vivido tu vida y espero vivir la mía. "Brocolita: alcanzarás las estrellas"; Pame, jamás podré expresarte toda mi gratitud por la confianza, la motivación, los consejos, los empujones, por ser mi mejor amiga; y mi admiración por ser mi modelo a seguir y la mujer más fuerte que conozco. ¡Y Gaby, gracias por ser parte nuestros sueños y de nuestra familia! Mi querida familia, abuelita, tío Viche, tía Betty, tío Gonzalito, Jorge Luis, Mayri, Gonza y los que se nos fueron antes de tiempo. Gracias por apoyarme en cada paso. Esta celebración nos incluye a todos, porque todos han sido mi inspiración. Simplemente gracias por una vida entera de felicidad. Como hubiera querido que este día estén a mi lado; tengan la certeza de que estarán en mi corazón cada minuto. La distancia siempre será dura, pero encontraremos siempre la manera de acortar el camino.

¡Mis carnalitas! ¡Me hacen falta mis cómplices! Nuestros recuerdos, sonrisas y el kit de supervivencia del último día siguen siendo mi mejor compañía. Carlita, Ale, gracias por una amistad eterna. Juanjo, Dani, Berni, Mari, compartimos los mismos 
sueños y nos despedimos el mismo año a distintos rincones del mundo a cumplirlos, ¡ya estamos todos muy cerca! Y a mis amigos Tomás, lo que construimos en los años más inocentes de nuestras vidas permanecerá en mi corazón para siempre. En este pequeño homenaje al pasado quiero mencionar al Dr. Carlos Cornejo y Dr. Fernando Serrano, gracias por inspirarme tanto siendo una niña.

“¡Se hace camino al andar!” ¡Pero más que el camino y el horizonte, lo que cuenta es la compañía!

To my future family-in-law, I am sure there is a beautiful expression in Farsi to express my love and gratitude towards you, and I hope I will be able to express it with my own words in the future. Thanks for joining my life, welcoming me into yours, and starting the beautiful adventure of planning a joint future. This book and this effort are also dedicated to you. Sannaz, Mohammad Reza, and Mehdi thanks for this translation:

$$
\begin{aligned}
& \text { خانو اده عزيز همسر آينده ام، مطمئن هستم كه جملات زيبايى در فارسى براى ابرازعلاقه من به شما وجود دارد و }
\end{aligned}
$$

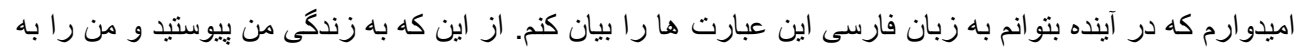

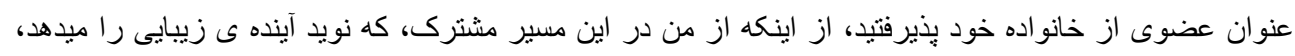

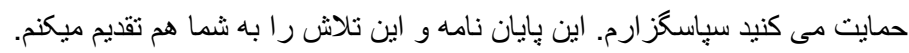

Behzad, I was not quite sure where to place you in this gratitude statement. You were there from the first day, welcoming me in the office with your kind smile. Our chemistry was almost inevitable. In a journey as exciting as this $\mathrm{PhD}$; you became my favourite colleague, my best friend, my roommate, and my partner. You are coauthor of a chapter of this book and of this chapter of my life. And on the last day of this journey you took the first step to the next one in our lives. You are absolutely my greatest adventure!

Undoubtedly some names escape my mind while writing this text. My apologies! You can be sure that if we shared even a single memory during the last five years, you are an important part of the pages of this book.

Respectfully, I extend my gratitude to my Thesis Committee for the time spent reviewing my work.

And to finish this section, to you dear reader, thanks for taking the time to read my book until the very last page (or from the last page)! I hope you liked the content and that it provided an idea on how quinoa is, out of many others, one of the hidden treasures in the Andean mountains.

Viviana, Wageningen, November, 2020 



\section{About the author}

Viviana Jaramillo Roman was born in Quito, Ecuador, on November 25, 1988. After receiving her high school diploma in natural sciences, she attended Universidad San Francisco de Quito (USFQ) with a scholarship for Academic Excellence. She obtained her bachelor degree as Engineer in Biotechnological Processes with honours in 2013. Her bachelor's thesis aimed to detect novel genes for salt tolerance in the Andean fruit Solanum betaceum. From 2011 to 2015 she worked as a research assistant in the

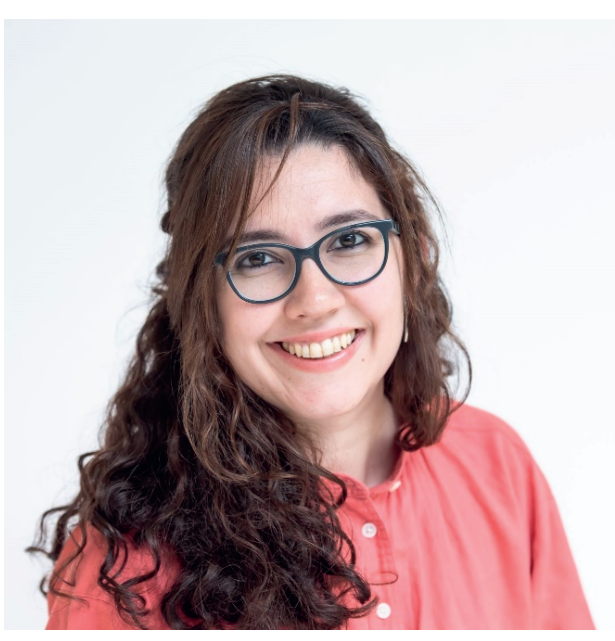
Plant Biotechnology Lab at USFQ, where she participated in several research projects mostly oriented to unveil the genetic diversity of several underutilized species with economic potential for the Ecuadorian markets. While working at USFQ, she developed a special interest in GMO management and genetic resources regulations, which encouraged her to participate as an assistant consultant for several government-led projects. She also pursued her passion for process engineering by obtaining a Green Belt Six Sigma certification. In 2014, she was awarded an Academic Excellence Scholarship from the Ecuadorian Government to pursue her doctorate studies. In October 2015, Viviana began her PhD studies at the Plant Breeding Laboratory under the supervision of prof. Richard Visser, $\mathrm{Dr}$ Gerard van der Linden and Dr Robert van Loo. The results of her PhD project are described in this thesis. In November 2020 she started working as a Project Manager in R\&D at Radicle Crops, a company that aims to transform quinoa into a global commodity.

linkedin.com/in/viviana-jaramilloroman 



\section{Education Statement of the Graduate School Experimental Plant Sciences}

Issued to: Viviana Jaramillo Roman

Date: 19 January 2021

Group: Laboratory of Plant Breeding

University: Wageningen University \& Research

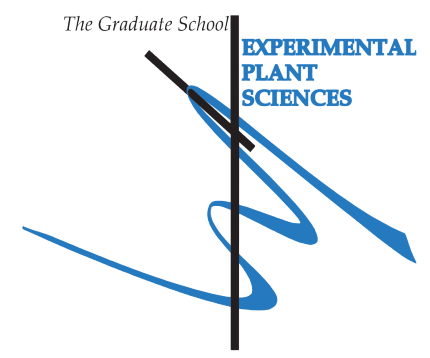

1) Start-Up Phase

- First presentation of your project

Salt tolerance mechanisms in quinoa

13 Nov 2016

- Writing or rewriting a project proposal

Genetic and molecular analysis of salt stress tolerance in

21 Feb 2017 quinoa

- Writing a review or book chapter

- MSc courses

2) Scientific Exposure

7.5 credits $^{*}$

- EPS PhD student days

EPS PhD student days 'Get2Gether 2017', Soest, NL

9-10 Feb 2017

EPS PhD student days 'Get2Gether 2018', Soest, NL

15-16 Feb 2018

- EPS theme symposia

EPS Theme 3 Symposium: 'Metabolism and adaptation',

Wageningen, NL

EPS Theme 3 Symposium: 'Metabolism and adaptation',

14 Mar 2017

Wageningen, NL

13 Mar 2018

- NWO Lunteren days and other National Platforms

NOW-ALW meeting 'Experimental Plant Science', Lunteren, NL

10-11 Apr 2017

NOW-ALW meeting 'Experimental Plant Science', Lunteren, NL

9-10 Apr 2018

NOW-ALW meeting 'Experimental Plant Science', Lunteren, NL

8-9 Apr 2019

- Seminars (series), workshops and symposia

EPS Flying Seminar: Prof. dr. Jane Parker, "Plant intracellular

21 Jan 2016

immunity: evolutionary and molecular underpinnings",

Wageningen, NL

EPS seminar: Dr. Sotirios Fragkostefanakis, "Alternative

2 Nov 2016

splicing of a heat stress transcription factor mediates

thermotolerance in tomato", Wageningen, NL

EPS Flying Seminar: Dr. Gerben van Ooijen, "Clocks across

taxa: Conserved cellular timekeeping in plants, algae and other

29 May 2017

eukaryotes", Wageningen, NL

Symposium: Genotype to Phenotype modelling of Plant

16 Nov 2017

Adaptation, Wageningen, NL

Working Conference: Models, improving photosynthesis and

21 Jun 2018

better crop yields: is this the future?, Wageningen, NL 
Symposium: Breeding data: Statistical Advances in Modern

16 Oct 2018

Plant Breeding, Wageningen, NL

Symposium: Plant Breeding and Biotechnology, Wageningen,

11-13 Jun 2019

$\mathrm{NL}$

Symposium: Land Plant Evolution \& Improving photosynthesis and crops, Wageningen, NL

Seminar: Dr. Monika Doblin, "Designing walls for a sustainable future", Wageningen, NL

Seminar: Prof. Mark Tester, "Genetic characterisation of salinity tolerance traits to increase salinity tolerance in crops",

Wageningen, NL

Workshop: Breeding for diversity, Wageningen, NL

20 Jun 2019

5 Jul 2019

12 Sep 2019

31 Oct 2019

Workshop \& 3rd WURomics symposium, Wageningen, NL

Mini-Symposium: Optimising photosynthesis for societal needs, Wageningen, NL

- Seminar plus

- International symposia and congresses

Gordon Research Seminar on Salt and Water Stress in Plants, Waterville Valley $\mathrm{NH}$, USA

Gordon Research Conference on Salt and Water Stress in

plants, Waterville Valley $\mathrm{NH}$, USA

Keystone Symposia Climate Change-Linked Stress tolerance in plants, Hannover, Germany

2nd International Quinoa Research Symposium, online event

2-3 Jun 2018

3-8 Jun 2018

13-16 May 2019

17-19 Aug 2020

\section{- Presentations}

Posters:

Plant and Animal Genome XXV Conference: "Genetic variation

14-18 Jan 2017

for salt tolerance in European sweet and bitter quinoa and their offspring", San Diego CA, USA

GRC on Salt and Water Stress in plants: "Physiological

responses of European sweet quinoa varieties to severe

10-12 Dec 2019

10 March 2020

salinity stress", Waterville Valley $\mathrm{NH}$, USA

NOW-ALW meeting Experimental Plant Science: "Comparison

of salt tolerance traits in quinoa grown under mild and severe

salt stress", Lunteren, NL

Keystone Symposia Climate Change-Linked Stress tolerance in

plants: "QTL mapping for salt tolerance traits in quinoa",

Hannover, Germany

Oral presentations:

NOW-ALW meeting Experimental Plant Science: "Salt stress

tolerance in quinoa: physiological adaptations of a halophytic

9-10 Apr 2018

food crop", Lunteren, NL

GRS on Salt and Water Stress in plants: "Physiological

2-3 Jun 2018

responses of European sweet quinoa varieties to severe salt

3-8 Jun 2018

8-9 Apr 2019

13-16 May 2019 stress", Waterville Valley NH, USA

- IAB interview

- Excursions 
3) In-Depth Studies

- EPS courses or other PhD courses

EPS: The power of RNA-Seq, Wageningen, NL

EPS PhD Summer School: Environmental Signaling in Plants, Utrecht, NL

PE\&RC: Introduction to $\mathrm{R}$ for statistical analysis, Wageningen, $\mathrm{NL}$

van Leeuwenhoek Centre for Advanced Microscopy: Basics in Fluorescence Microscopy, Amsterdam, NL

EPS: Data Analysis and Visualization in R, Wageningen, NL

VLAG\&EPS: Microscopy and Spectroscopy in Food and Plant Sciences, Wageningen, NL

ELIXIR-EXCELERATE, NIOO-KNAW, UvA, EPS: Software

Carpentry Workshop on Python programming, Amsterdam, NL

ELIXIR-EXCELERATE, NIOO-KNAW, UvA, EPS: The

Carpentries Workshop: Genomics Data, Wageningen, NL

\section{Journal club}

Individual research training
10-12 Feb 2016

$\underline{\text { date }}$

28-30 Aug 2017

23-24 Oct 2017

6-9 Feb 2018

8-9 May 2018

14-16 May 2018

5-6 Feb 2019
14-15 Jan 2019

\section{Subtotal In-Depth Studies}

6.5 credits $^{*}$

4) Personal Development

$\underline{\text { date }}$

\section{Skill training courses}

EPS: EPS Introduction course, Wageningen, NL

29 Sep 2016

In'to languages: Presenting with Impact, Wageningen, NL

Nov \& Dec 2016

WU\&Research Library: Scientific Artwork- Vector graphics and

2-3 Oct 2017 images, Wageningen, NL

In'to languages: Scientific Writing, Wageningen, NL

Apr \& Jun 2018

- Organisation of PhD students day, course or conference

Membership of Board, Committee or PhD council

Subtotal Personal Development

3.7 credits $^{*}$

TOTAL NUMBER OF CREDIT POINTS*

37.6

Herewith the Graduate School declares that the PhD candidate has complied with the educational requirements set by the Educational Committee of EPS with a minimum total of 30 ECTS credits.

${ }^{*}$ A credit represents a normative study load of 28 hours of study. 

Viviana Jaramillo Roman was financially supported by a scholarship from the Secretariat for Higher Education, Science, Technology and Innovation of the Republic of Ecuador (Contract Number 2015-AR2Q8878).

Financial support from Wageningen University for printing this thesis is greatly appreciated.

Cover painting: Marcelo Chamorro

Book layout: Behzad Rashidi

Printed by Proefschriftmaken on FSC-certified paper 



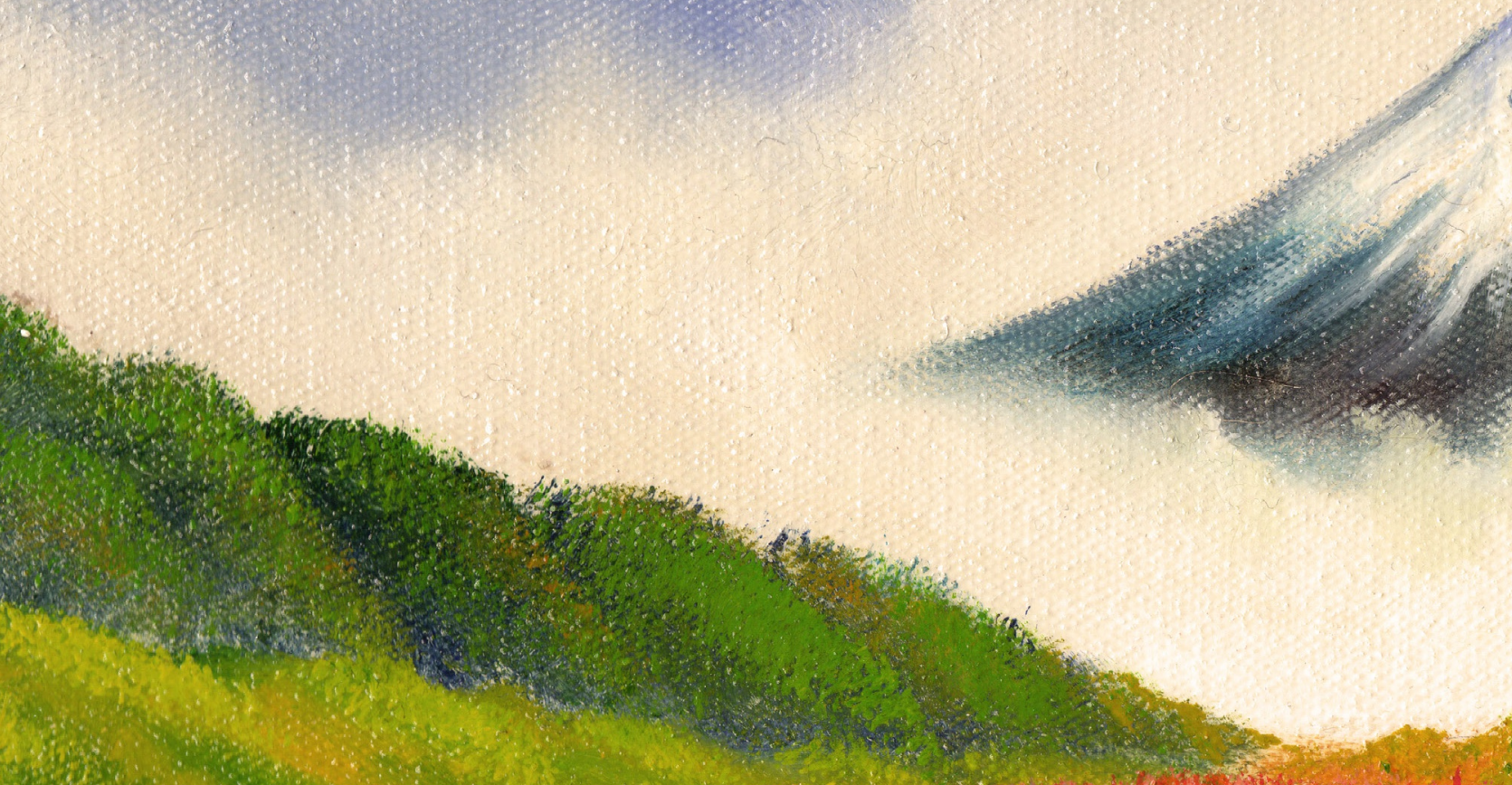

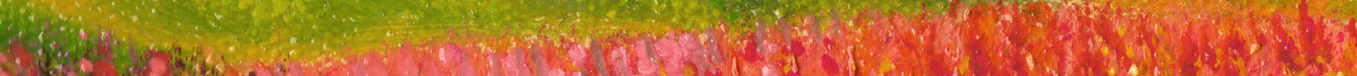

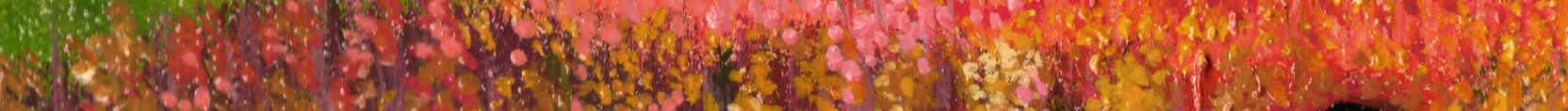

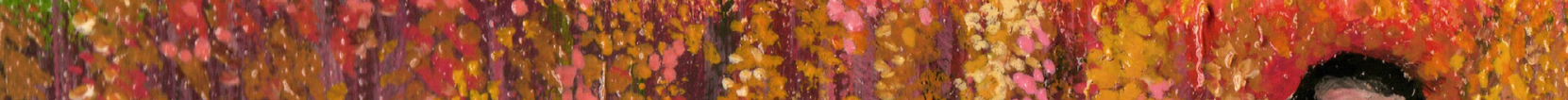

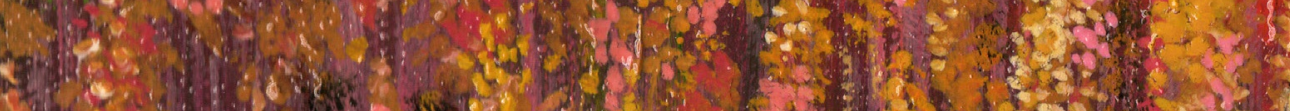

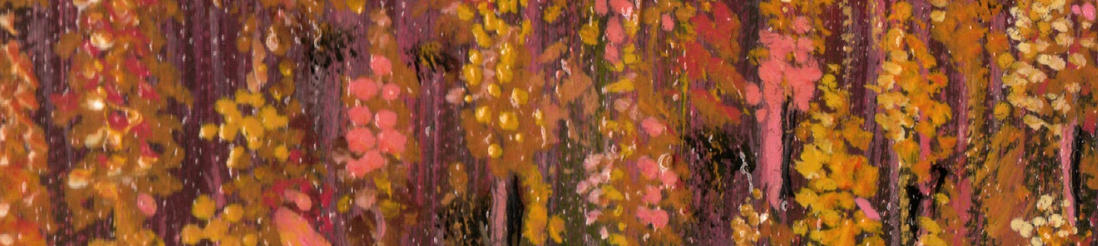

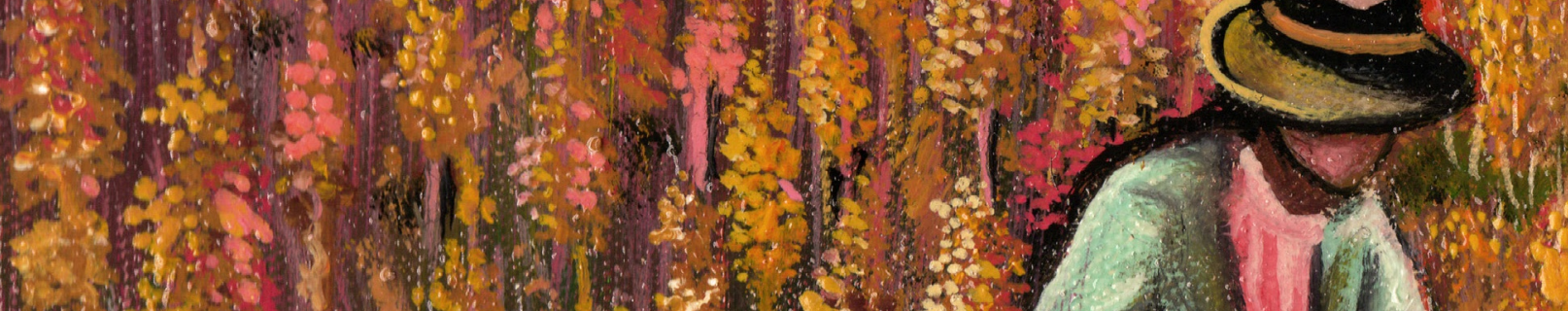

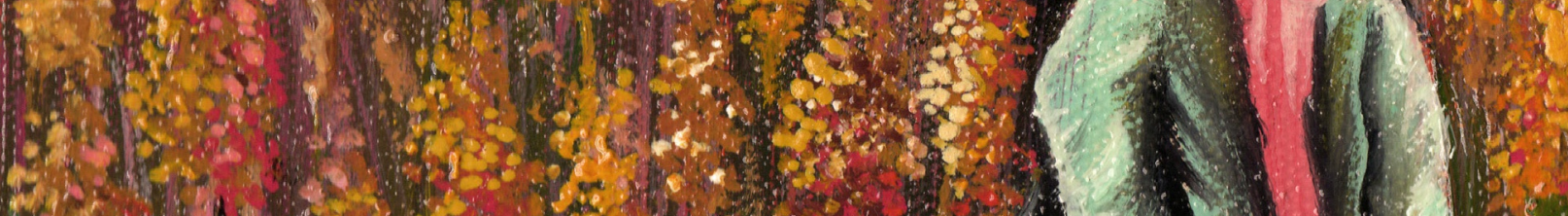

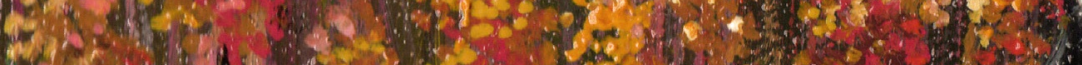

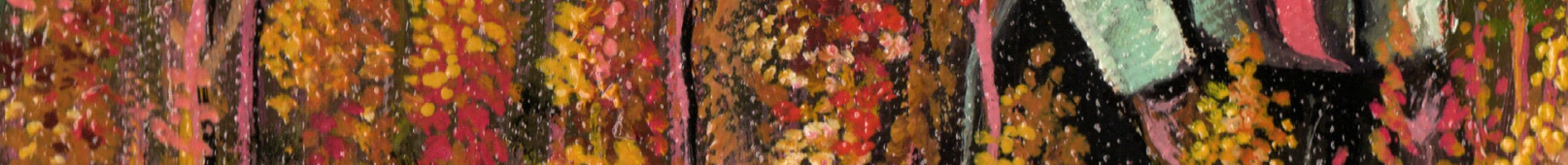

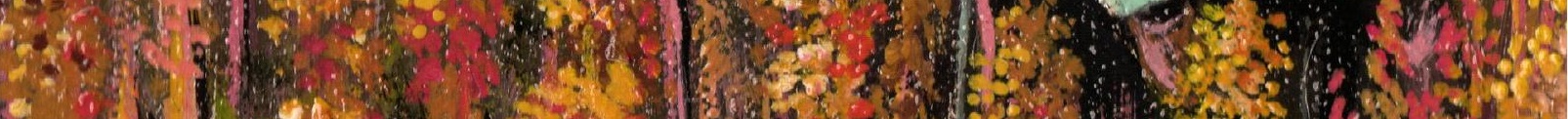

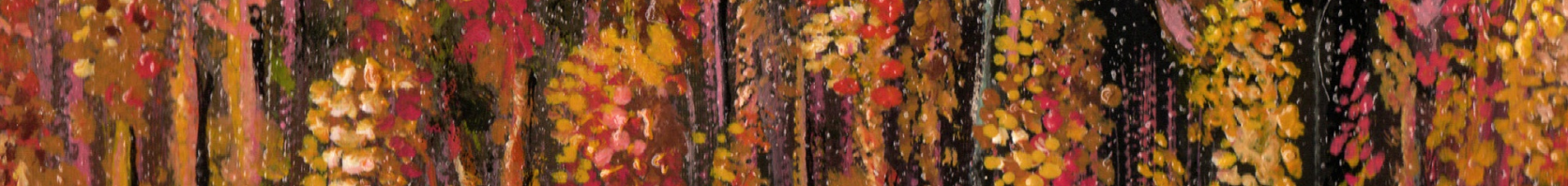

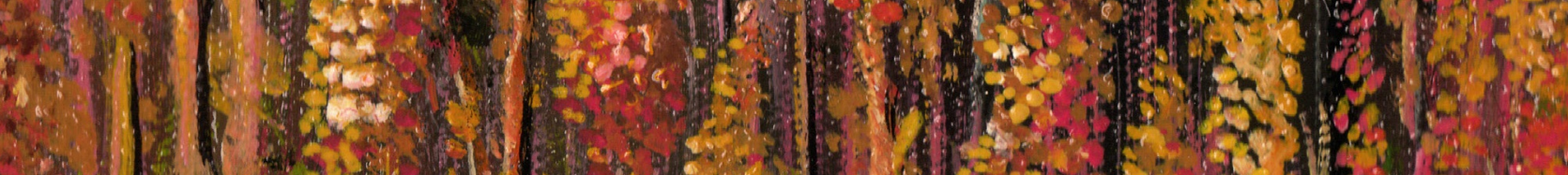

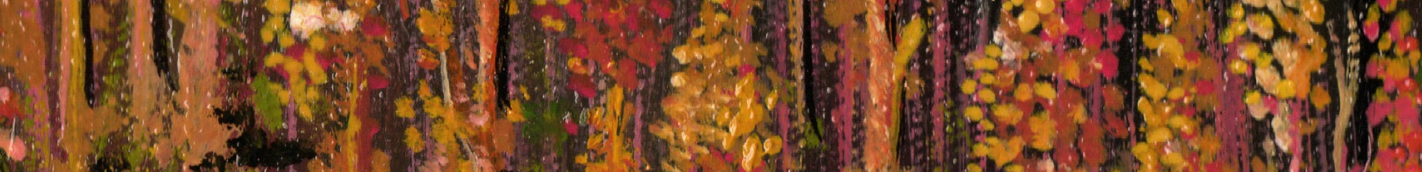
- 